\title{
Two-Dimensional Barcodes for Mobile Phones
}

\author{
by
}

\section{Sarah Lyons}

\author{
A thesis submitted in conformity with the requirements \\ for the degree of Master of Applied Science \\ Graduate Department of Electrical \& Computer Engineering \\ University of Toronto
}

(C) Copyright by Sarah Lyons 2009 


\author{
Abstract \\ Two-Dimensional Barcodes for Mobile Phones \\ Sarah Lyons \\ Master of Applied Science \\ Graduate Department of Electrical \& Computer Engineering \\ University of Toronto \\ 2009
}

There are several potential applications for a high data density barcode that can be easily photographed and decoded by mobile phones, but no such symbology currently exists. As a result, a new barcode was designed to exploit the low-pass characteristic of a camera phone channel and is presented as a means of facilitating wireless optical communication with mobile phones.

A channel model was established and subsequent simulation results led to the design of a colour barcode with encoding done in the Discrete Cosine Transform domain. A waterfilling process and a noise-shaping algorithm enhance performance, while a new fast acquisition method allows for rotational and size invariance. An outer Accumulate-RepeatAccumulate code is employed, followed by an inner Reed Muller code with a rate varying according to spatial frequency.

The final barcode data-density is 3.5 times greater than the leading symbology and has proven robust to various impediments imposed by camera phones. 


\section{Acknowledgements}

I wish to extend my gratitude to all people who have helped and inspired me during my Masters study. I especially want to thank my supervisor, Dr. F.R. Kschischang, who was always accessible and willing to help and offer suggestions. His guidance helped make my research a very rewarding experience.

The encouragement and financial support of the Electrical and Computer Engineering Department at the University of Toronto were invaluable in initiating and nurturing my research work. I would also like to thank the Ontario Graduate Scholarship Program for a 2007-2008 OGS scholarship and the Natural Sciences and Engineering Research Council of Canada (NSERC) for a 2008-2009 CGS scholarship.

Finally, I would like to thank my friends and family for their endless support and encouragement. 


\section{Contents}

1 Introduction $\quad 1$

2 Background 3

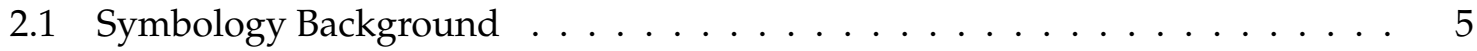

2.1.1 One-Dimensional/Linear Symbologies . . . . . . . . . . . . . 5

2.1.2 Two-Dimensional Barcodes . . . . . . . . . . . . . . . . . 7

2.2 Specifics of Notable Two-Dimensional Barcodes . . . . . . . . . . . . 10

2.2 .1 INTACTA.CODE . . . . . . . . . . . . . . . . . . . . 10

2.2 .2 Data Matrix . . . . . . . . . . . . . . . . . 11

2.2 .3 QR Code ................................ 13

2.2.4 HCCB (High Capacity Color Barcode) . . . . . . . . . . . . . . . 14

2.2 .5 Data Glyphs . . . . . . . . . . . . . . . . . . 16

2.2 .6 Granite Codes . . . . . . . . . . . . . . . . . . . . . 17

2.3 Symbology Comparison . . . . . . . . . . . . . . . . . 19

2.4 Research Phases . . . . . . . . . . . . . . . . . . . . . . 20

3 Mathematical Channel Model $\quad 22$

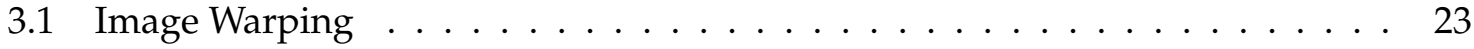

3.2 Point Spread Function $(\mathrm{PSF}) \ldots \ldots \ldots \ldots \ldots$

3.2 .1 Diffraction . . . . . . . . . . . . . . . . . . 28

3.2.2 Fourier Transforming Property of a Thin Lens . . . . . . . . . . . 32

3.2.3 The PSF and OTF of a Thin Lens . . . . . . . . . . . . . . 35

3.2 .4 Defocused Images $\ldots \ldots \ldots \ldots$

3.2 .5 Motion Blur . . . . . . . . . . . . . . . . . . . . . . . . 39 
3.2.6 Overall Optical Transfer Function . . . . . . . . . . . . . . . 40

3.3 CMOS Camera Response . . . . . . . . . . . . . . . . . . . . 43

3.3 .1 CMOS Imagers . . . . . . . . . . . . . . . . . . . . . 43

3.3.2 Nyquist Sampling Theory and Cut-Off Frequency . . . . . . . . . . . . 46

3.3 .3 Sampling . . . . . . . . . . . . . . . . . 47

3.3.4 Nonidealities and Dynamic Range . . . . . . . . . . . . . . . . . 50

3.3.5 Modelling the Dynamic Range . . . . . . . . . . . . . . . . . 53

3.3.6 Colour Filter Imperfections and Cross-Talk . . . . . . . . . . . . . 56

3.3.7 The Overall CMOS Distortion Function . . . . . . . . . . . . . . . . 56

3.4 Camera Processing . . . . . . . . . . . . . . . . . . . . . . 59

3.4.1 Gamma Correction . . . . . . . . . . . . . . . . . . . . . . . 60

3.4 .2 Demosaicing ........................... 61

3.4.3 Colour Correction and Other Processing . . . . . . . . . . . . 63

3.5 Additive Noise . . . . . . . . . . . . . . . . . . . . . . . . 64

3.6 JPEG Compression . . . . . . . . . . . . . . . . . . 65

3.7 Transmitter Nonlinearities . . . . . . . . . . . . . . . . . . . . . . 69

3.8 Areas of Uncertainty $\ldots \ldots \ldots \ldots \ldots \ldots$

3.9 Preliminary Tests . . . . . . . . . . . . . . . 70

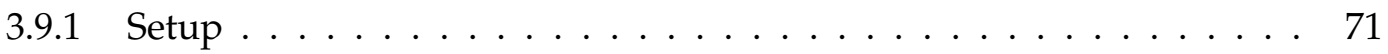

3.9.2 Black and White versus Colour . . . . . . . . . . . . . 72

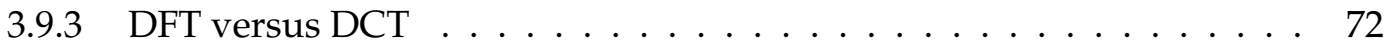

4 MATLAB Channel Simulator $\quad 75$

4.1 MATLAB Transmitter Nonlinearity . . . . . . . . . . . . . . 76

4.2 MATLAB Warping Function . . . . . . . . . . . . . . . 76

4.3 MATLAB Lens PSF Function $\ldots \ldots \ldots \ldots \ldots$

4.4 MATLAB CMOS Camera Response . . . . . . . . . . . . . . . . . . 80

4.5 MATLAB Camera Processing and Compression . . . . . . . . . . . 80

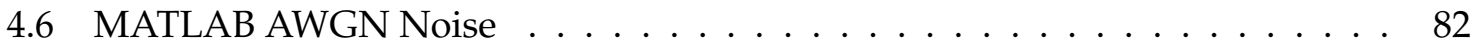

4.7 Channel Uncertainty . . . . . . . . . . . . . . . . . . . . 83

4.7.1 Identifying the Variable Parameters . . . . . . . . . . . . . . . . . 84 
4.7.2 Defining the Range of Variation . . . . . . . . . . . . . . . . 84

4.7 .3 The Limiting Cases . . . . . . . . . . . . . . . . . . . . . 87

4.8 Simulator Adjustments and Histogram Matching $\ldots \ldots$. . . . . . . . . 88

4.9 Mutual Information Calculations . . . . . . . . . . . . . . . . . . . . 89

4.9 .1 Experimental Setup . . . . . . . . . . . . . . . . . . . 90

4.9 .2 Binning Method . . . . . . . . . . . . . . . . . 90

5 Digital Signal and Image Processing $\quad 94$

5.1 Processing at the Transmitter . . . . . . . . . . . . . . . . 94

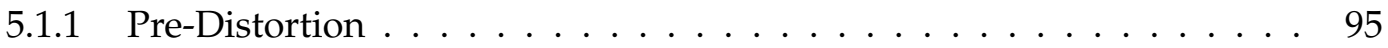

5.1 .2 Noise Shaping . . . . . . . . . . . . . . . . . . . . . 96

5.1 .3 Power Allocation . . . . . . . . . . . . . . . . . . . . . . . 99

5.2 Processing at the Receiver . . . . . . . . . . . . . . . . 102

5.2 .1 The Fast DCT . . . . . . . . . . . . . . . . . . . 103

5.2 .2 Acquisition . . . . . . . . . . . . . . . . . . 104

5.2 .3 Perspective Correction . . . . . . . . . . . . . . . . . . . . 109

5.2.4 Gamma Correction . . . . . . . . . . . . . . . . . . . . . . 112

5.2 .5 Contrast Stretch . . . . . . . . . . . . . . . . . . . . . 113

5.2 .6 Colour Correction $\ldots \ldots \ldots \ldots$

5.2 .7 Gain Adjustments . . . . . . . . . . . . . . . . . . . . . 113

5.2.8 Calibration and Other Image Processing . . . . . . . . . . . . . . 114

5.3 Estimated Capacity . . . . . . . . . . . . . . . . . . . . 115

6 Modulation and Coding $\quad 117$

6.1 Modulation and Demodulation . . . . . . . . . . . . . . . . . . 121

6.1 .1 Modulation and Mapping . . . . . . . . . . . . . . . 121

6.1.2 Demodulation and De-Mapping . . . . . . . . . . . . . . . 122

6.2 Inner Reed Muller Code . . . . . . . . . . . . . . . . . . . . . . . . 124

6.2 .1 Inner Encoding . . . . . . . . . . . . . . . . . . . . . . 124

6.2 .2 Inner Decoding . . . . . . . . . . . . . . . . . . 125

6.3 Outer ARA Code . . . . . . . . . . . . . . . . . . 128 
7 Final Design and Results $\quad 135$

7.1 Design Summary . . . . . . . . . . . . . . . . . . . . . . 135

7.2 Tests and Results . . . . . . . . . . . . . . . . . . . . . 140

7.3 Suggestions for Future Work . . . . . . . . . . . . . . . . . . . . . . 141

8 Conclusion $\quad 145$

$\begin{array}{ll}\text { A Data-Density Calculations } & 147\end{array}$

$\begin{array}{ll}\text { B Motorola Camera Phone Specifications } & 150\end{array}$

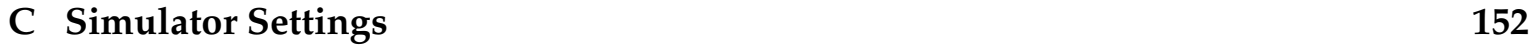

$\begin{array}{ll}\text { D Processing } & 161\end{array}$

$\begin{array}{lll}\text { E Capacity Estimates } & 165\end{array}$

$\begin{array}{lll}\text { F } & \text { MAP Check Sum Approximation } & 169\end{array}$ 


\section{List of Tables}

2.1 Density of Notable Barcode Symbologies at 600 dpi. . . . . . . . . . . . . . . 19

3.1 Typical Gamma Values . . . . . . . . . . . . . . . . . . . 50

3.2 Characteristics for Typical CMOS Pixels in Mobile Phones . . . . . . . . . . 55

4.1 Variable Parameters in the Overall Channel Model . . . . . . . . . . . . . 85

4.2 Range Over which Variable Channel Parameters are Defined . . . . . . . . . . 86

6.1 Golden Dithered Interleaver Parameters used for ARA Code . . . . . . . . 133

7.1 RGB DCT Barcode Design Specifications . . . . . . . . . . . . . . . . 136

7.2 Software Receiver Complexity for $400 \times 400$ Pixel Barcode . . . . . . . . . 138

C.1 Simulator Settings for Mutual Information Calculations . . . . . . . . . . . 153 


\section{List of Figures}

2.1 List of Common Barcode Symbologies $[1,2,3,4] \ldots \ldots$

2.2 INTACTA.CODE Symbol . . . . . . . . . . . . . . . . . . . . . . 11

2.3 Data Matrix Symbol . . . . . . . . . . . . . . . . . . . . . . . . . 12

2.4 QR Code Symbol . . . . . . . . . . . . . . . . . . . . . 13

2.5 HCCB Symbols . . . . . . . . . . . . . . . . . . . . . . . 15

2.6 Data Glyph Symbol . . . . . . . . . . . . . . . . . . . . . 16

2.7 Data Glyph Symbol with Data Toning . . . . . . . . . . . . . . . . 16

2.8 Granite Code by Gupta and Kschischang . . . . . . . . . . . . . . . . 18

3.1 Basic Channel Pictorial Diagram . . . . . . . . . . . . . . . 22

3.2 Barcode Skewing . . . . . . . . . . . . . . . . 24

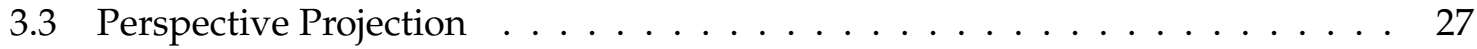

3.4 Spherical vs. Planar Wave Patterns . . . . . . . . . . . . . . . . . . . . . . . . 29

3.5 Plane Wave Tilt from Optic Axis . . . . . . . . . . . . . . . . . 30

3.6 Basic Diffraction Geometry . . . . . . . . . . . . . . . 30

3.7 Thin Lens with Real Image . . . . . . . . . . . . . . . . . . . . 33

3.8 Lens Geometry ～. . . . . . . . . . . . . . . . . . . . . . . . . . 33

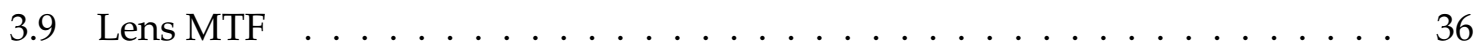

3.10 Formation of a Defocused Image . . . . . . . . . . . . . . 37

3.11 MTF due to Defocus $\ldots \ldots \ldots \ldots \ldots \ldots \ldots$

3.12 MTF Due to Motion Blur . . . . . . . . . . . . . . . . . . . . . 40

3.13 Overall Transfer Function of Camera Lens . . . . . . . . . . . . . . . . . . 41

3.14 CMOS Image Sensor . . . . . . . . . . . . . . . . . . . . 44

3.15 CMOS with Microlens and CFA . . . . . . . . . . . . . . . . . . . . 44 
3.16 Illustration of the Photoelectric Effect . . . . . . . . . . . . . . . . . . 46

3.17 CMOS Dynamic Range . . . . . . . . . . . . . . . . . . . . . 51

3.18 CMOS Noise Under Dark and Illuminated Conditions . . . . . . . . . . . . . 53

3.19 CMOS Image Sensor Pixel Model … . . . . . . . . . . . . . . . . . . . 54

3.20 MTF of a Given CMOS Sensor Array . . . . . . . . . . . . . . . . . 57

3.21 Scope of the SMIA Standard . . . . . . . . . . . . . . . . . 5 59

3.22 Gamma Curves . . . . . . . . . . . . . . . . . . . . 60

3.23 Demosaicing . . . . . . . . . . . . . . . . . 62

3.24 Spectral Response of a Typical Bayer Filter . . . . . . . . . . . . . . . . . 64

3.25 Typical JPEG Normalization Array, $Z(u, v) \ldots \ldots$. . . . . . . . . 68

3.26 Granite-like, OFDM Barcode used in Preliminary Tests . . . . . . . . . . 72

3.27 RGB version of Barcode used in Preliminary Tests . . . . . . . . . . . 73

3.28 A DCT version of the RGB barcode . . . . . . . . . . . . . . . . . 74

4.1 Linear Spatial Filtering Process _ . . . . . . . . . . . . . . . . . . 78

4.2 Number of Samples vs. Capacity Measurements . . . . . . . . . . . . . . . 92

4.3 Capacity Estimation Method . . . . . . . . . . . . . . . . 93

5.1 Block Diagram of Transmitter Processing . . . . . . . . . . . . . . . 95

5.2 Error Diffusion System . . . . . . . . . . . . . . . . . . . 96

5.3 Error Diffusion Example . . . . . . . . . . . . . . . . 97

5.4 Projection onto Convex Sets . . . . . . . . . . . . . . . . . . . . . . 98

5.5 Block Diagram of Projection onto Convex Sets Method . . . . . . . . . . . 98

5.6 Noise Histograms for Different Frequency Bins ～. . . . . . . . . . . . 100

5.7 Block Diagram of Receiver Processing . . . . . . . . . . . . . . . . 102

5.8 Fiducials for Proposed Acquisition Method ～. . . . . . . . . . . . . 106

5.9 Pattern for Receiver Correlation . . . . . . . . . . . . . . . . . . . . . 106

5.10 Estimated Capacity, truncated . . . . . . . . . . . . . . 115

6.1 Transmitter Block Diagram (Encoder, Modulator) . . . . . . . . . . . 118

6.2 Receiver Block Diagram (Decoder, Demodulator) . . . . . . . . . . . . . 118

6.3 Frequency Regions, Red/Blue . . . . . . . . . . . . . . . . . . . . . . . . 119

6.4 Frequency Regions, Green . . . . . . . . . . . . . . . . . . . . . . 120 
6.5 A Basic ARA Encoding Structure . . . . . . . . . . . . . . . . . . . 128

6.6 Family of Multi-edge Type ARA Codes . . . . . . . . . . . . . . . . . . . . . 129

6.7 Protograph for Multi-Edge Family of ARA Codes ～. . . . . . . . . . . 130

6.8 Factor Graph for Rate $1 / 2$ Multi-Edge ARA Code . . . . . . . . . . . . . . 131

6.9 Protograph for Rate $1 / 2$ Multi-Edge ARA Code . . . . . . . . . . . . . . . 131

6.10 Interleaver from Figure 6.8 with Edge Classes Numbered . . . . . . . . . . 132

7.1 Final RGB DCT Barcode . . . . . . . . . . . . . . . . . . . . . . . . . 137

7.2 Complexity Breakdown . . . . . . . . . . . . . . . . . . . . . . . 139

7.3 Ratio of Received Pixels to Transmitted Pixels versus Capacity . . . . . . . . 142

7.4 Framing Barcode in Phone LCD . . . . . . . . . . . . . . . . . . . . 144

B.1 MOTOROKR E6/E6e Developer Reference Sheet . . . . . . . . . . . . . . . 151

C.1 Original Barcode Transmitted (see Figure C.2) … . . . . . . . . . 154

C.2 Simulated Scenario for Barcode Display . . . . . . . . . . . . . . . . 155

C.3 Simulated Photograph of Barcode (with Gray-World AWB) . . . . . . . . . 155

C.4 Resultant Received Barcode . . . . . . . . . . . . . . . . . . . . . . . 156

C.5 Original Transmitted Barcode (see Figures C.6 - C.11) _ . . . . . . . . 157

C.6 Simulated Photograph of Barcode . . . . . . . . . . . . . . . . . . . 158

C.7 Simulated Photograph of Barcode . . . . . . . . . . . . . . . . . . 158

C.8 Simulated Photograph of Barcode . . . . . . . . . . . . . . . . . . 159

C.9 Simulated Photograph of Barcode . . . . . . . . . . . . . . . . . . . 159

C.10 Simulated Photograph of Barcode . . . . . . . . . . . . . . . . . . . 160

C.11 Simulated Photograph of Barcode . . . . . . . . . . . . . . . . . . . . . 160

D.1 Original Barcode Transmitted (see Figure D.2) $\ldots \ldots \ldots \ldots \ldots$

D.2 Simulated Photograph of Barcode . . . . . . . . . . . . . . . . 162

D.3 Acquired Barcode after Perspective Correction . . . . . . . . . . . . . 163

D.4 Received Image with Fiducials Eliminated . . . . . . . . . . . . . . . 163

D.5 Received Image after Gamma Correction ．. . . . . . . . . . . . . . . . 164

D.6 Received Image After Global Spatial Domain Gain Adjustment . . . . . . . 164

E.1 Full-scale Estimated Capacity . . . . . . . . . . . . . . . . . . 166 
E.2 Full-scale Estimated Capacity, no Fiducials . . . . . . . . . . . . . . . 167

E.3 Noise Power in DCT Domain . . . . . . . . . . . . . . . . . . . . 168

F.1 True Check Sum Function ． . . . . . . . . . . . . . . . . . . . 170

F.2 Check Sum Approximation . . . . . . . . . . . . . . 170 


\section{Chapter 1}

\section{Introduction}

As the information technology industry continues to grow at a rapid rate, digital communications have become prevalent in every domain of life. The virtual world is ever-expanding. However, a considerable portion of business, advertising, and logistics depends heavily on physical media for communication. Despite the prominence of digital devices and computers in the business world, paper continues to play a significant role. For this reason, methods of interfacing documents and objects with digital formats and virtual databases can significantly increase efficiencies and enhance communication. Perhaps the most recognizable of such methods is the barcode.

Since their commercial debut in the 1960's, barcodes have become essential to many industries and are now omnipresent in our world. A barcode is a machine-readable representation of information displayed on a surface. Generally, barcodes use dark markings on a light surface to create a high and low reflectance that can be converted into binary digits. However, several techniques have diverged from the traditional encoding methods by using colour, as well as representations that are invisible to the human eye. Barcodes are read using optical scanners, or decoded from images using the required software $[1,5]$.

The original one-dimensional barcodes encode several characters into a linear series of bars. Because of their low data-density, these linear barcodes are used to simply link an item to an existing database when scanned. For this reason, they are termed license plate files. The Universal Product Code (UPC) is one such barcode that plays a crucial role in facilitating the trade of commercial items, such as groceries.

The desire to encode more data into a small spatial region motivated several advance- 
ments in barcode technology. Two-dimensional barcodes were developed in the mid-eighties in order to encode all of the necessary information about an item into the barcode itself, as opposed to just its address in an existing database. Two-dimensional barcodes encode information along the vertical axis as well as the horizontal axis, while one-dimensional barcodes encode data in the horizontal direction only, with vertical redundancy.

Several types of two-dimensional barcode encoding schemes, called symbologies, have emerged over the past twenty years. Applications extend over several areas of business and government, including the labelling of electronics, health care equipment management, package tracking, as well as encoding information onto personal identification cards and passports. The data-densities achieved by some of today's symbologies are hundreds of times greater than those of the original linear barcodes.

There are trade-offs to consider when evaluating barcodes. A higher data-density generally comes at the cost of a higher decoding complexity as well as higher resolution printing and scanning requirements. Different symbologies of varying densities can be valuable for different applications. For this reason, barcodes are often designed with a particular application in mind. For example, the ArrayTag barcode is optimized to be read in variable lighting or at a distance and can be read from up to 50 meters away [1]. Other barcodes have been designed to blend into pictures or even be invisible to the human eye. In general, barcode symbologies attempt to incorporate powerful error-correction coding to increase the robustness of the code. This allows them to be decoded correctly in the presence of distortion and noise, which will inevitably arise from a variety of sources.

Although barcodes are already being used extensively in many fields, there are still limitless possibilities. Mobile ticketing and couponing solutions are currently being implemented through barcodes that can be captured and decoded by cell phones equipped with a digital camera. Recently, the drive to advertise corporate messages to consumers across every possible medium has prompted a new application of barcodes: advertising through barcodes scanned by cell phone cameras. 


\section{Chapter 2}

\section{Background}

Two-dimensional barcodes have proven to be an effective method of labelling where central databases are infeasible and physical surface area is limited. The potential roles of these 2-D barcodes have certainly not been exhausted and there are several promising applications emerging with the ever-expanding digital world.

Camera phones encompass one of the newest domains entered by the barcode, as well as one of the most exciting. Several recent press releases indicate the growing popularity of this application $[6,7,8,9,10,11]$. In April, 2007, BBC News presented the High Capacity Color Barcode (HCCB) for cell phone scanning, and indicated that the symbol would appear on DVDs within a year [6]. Fujitsu recently introduced an encoding technology that embeds data invisibly into a picture to be decoded specifically by mobile phones [10]. This was in response to aversion toward the obtrusive appearance of Quick Response (QR) symbols on advertisements. QR Code and Data Matrix decoders for cell phones are increasing in popularity, and a high percentage of Japanese people have used QR Codes for a cell phone application.

In order to capitalize on the barcode possibilities presented through personal wireless devices, such as cell phones and blackberries, many considerations must be made. Although a high data-density is appealing to corporations looking to advertise, the user experience must be taken into account. Cell phone processor sizes must be considered, as well as the distortion presented by capturing the barcode with the cell phone camera. In order to create a barcode system that is practical for use with a cell phone, it is imperative that the probability of decoding failure is low so that the consumer finds it easy and enjoyable 
to use.

Some recent reviews of barcode scanning and decoding with cell phones have been disappointing. It might be worth considering camera phone specifics when designing a symbology, as opposed to forcing an ill-suited symbology into the desired application. Although the HCCB shows promise in this domain and claims to be suited to camera phone applications, its data-density is inferior to some other symbologies despite its use of eight different colours.

Perhaps a combination of spatially bandwidth-efficient coding, the use of colour, and consideration to cell phone-specific applications could yield a superior code for this particular function. Investigations into this area are worthwhile, especially since the processing power of cell phones has been increasing, and will continue to do so.

There are other applications and potential symbology improvements. If 2-D barcode systems are to be adopted by industries to provide a simple and convenient interface between the paper and digital domains for their consumers, aesthetics will play a role. The ideal barcode is as flexible in size, shape and colour as possible. This will allow it to blend discreetly into packaging, advertisements, or company logos. Fixed architectures, fiducials, and other position locator markings lessen the appeal of most current symbologies.

There are many possibilities for 2-D barcodes in the near future. A barcode designed judiciously to have a high data-density, a reasonable decoding complexity, a flexible size and appearance, and sufficient robustness to noise and distortion has the potential to take barcodes from their specialized functions in industry to a universally employed interface between printed and digital domains.

The design of a data-dense, robust, flexible, and easily decodable 2-D barcode will allow barcode systems to penetrate new markets and succeed in new applications. Given the technology available, there are no obvious obstacles to achieving such a code, and therefore the R\&D process should not be delayed. Before delving into a new barcode design, it is worth looking at current symbologies and identifying their features and limitations. 


\subsection{Symbology Background}

The first barcode patent was issued to Joseph Woodland and Bernard Silver in 1952 [12, 13]. Since their major debut in grocery stores as the distinguished Universal Product Code (UPC), barcodes have evolved significantly and are prominent in our world today.

Barcode symbology refers to the mapping between the message and the barcode. The first barcodes employed one-dimensional symbologies, meaning that encoding is done in only one spatial dimension (along one axis). Two-dimensional symbologies revolutionized barcode technology by also encoding data in the second dimension of the surface. Today, further advancements are being made to take 2D symbologies away from their traditional encoding schemes in order to increase data-density and enhance performance.

\subsubsection{One-Dimensional/Linear Symbologies}

There are several distinguished linear barcodes in addition to the ubiquitous UPC, such as the well-known International Standard Book Number (ISBN) used to uniquely identify each edition of every published book and book-like product [14].

As illustrated in Figure 2.1, one-dimensional barcodes encode data along the horizontal axis through the use of bars and spaces. The specifications include the actual encoding of digits as well as the marking of bars and spaces. One-dimensional barcodes can be categorized as being discrete or continuous, as well as having two bar widths or many bar widths [15].

Continuous symbologies have characters adjoined, with one character ending in a bar and the next beginning in a space, or vice versa. Discrete symbologies use characters that begin and end with bars. The inter-character space is generally ignored.

Two-width symbologies have one designated narrow bar width and one wide bar width. The widths are specified in relative quantities, usually with the wide width being two or three times greater than the narrow, so there is no dependency on the absolute measurements. Many-width symbologies use bars and spaces that are all multiples of a specified width, called the module of the code.

One-dimensional barcodes are read using optical scanners, often called barcode readers. The scanners are either hand-held or fixed-mount. Handheld scanners are used for 
stationary items, while fixed-mount scanners require that the item be physically passed by the scanner. This is usually done by hand in retail applications and by conveyor belt in industrial applications [16]. In general, both handheld and fixed-mount scanners employ either a laser or a CCD (charged-coupled device) imager.

Laser scanners were the first type of barcode reader to be utilized. They use a laser diode to create an infrared beam which is spread in an arc parallel to the barcode by a rapidly rotating mirror. A photodiode is then employed to measure the intensity of the light reflected back from the barcode surface. More sophisticated scanners often use revolving polygons or oscillating mirrors to enhance performance. The optimal scanning distance for laser scanners is typically 6-12 inches, but can range up to 35 feet for certain reflective barcodes $[1,13]$.

CCD scanners use a stationary flood of light, usually LEDs (light emitting diodes), to reflect the symbol image back onto an array of photosensors. The optimal scanning distance usually ranges from physical contact to six inches. CCD scanners are generally less expensive than laser scanners and have a durability advantage, primarily because they contain no moving parts. As a result, CCD imagers are surpassing laser scanners as the preferred technology for reading linear barcodes.

Linear barcodes are used extensively in libraries, grocery stores, and almost all commercial environments. Their role of efficiently linking an item to its location in a database is expected to continue despite significant advancements in barcode symbologies. Higher density, two-dimensional barcodes are generally unnecessary for applications where linear barcodes currently dominate. However, RFID (Radio Frequency Identification) tags may offer advantages over barcodes to manufacturers in such domains.

If linear barcode systems are replaced in retail venues, RFID tags would probably be a more appropriate substitute than two-dimensional barcodes. RFID tags (also known as transponders) do not require a direct line of sight for reading and may be read through hard material such as book covers or other packaging material. Each tag can uniquely identify the object to which it is attached, even if that object is one of a multitude of identical items. However, RFID tags are more costly than barcodes and raise privacy concerns [17].

Linear barcodes play a well-established role in industry that is not currently threatened by higher density barcodes. However, there are a multitude of other applications for which 
linear barcodes are insufficient because a central database is simply not feasible, or surface area is limited.

\subsubsection{Two-Dimensional Barcodes}

In order to meet the demand for a higher data-density barcode system, two-dimensional symbologies were developed. The ability to encode a portable database in a limited spatial area has allowed barcodes to prevail in applications originally prohibited by the linear symbology. The health care industry benefited by being able to label unit-dose packages, with the labelling of other medicines and tools to follow. The electronics industry also began using two-dimensional barcodes to label small parts. Several other industries have followed with a variety of applications.

The two-dimensional concept was initiated in 1984 when the Automotive Industry Action Group introduced a standard for shipping and identification labels which consisted of four Code 39 barcodes (a linear symbology) stacked on top of each other. Then in 1988, Code 49 was introduced by the Intermec Corporation to become the first truly two-dimensional barcode on the market. Like the stacked Code 39 barcode, Code 49 also used the idea of layering linear barcodes along the vertical axis, as can be seen in Figure $2.1[2,18]$.

Several different two-dimensional barcodes have been introduced since Code 49 . For the most part, they can be categorized as having either a stacked or a matrix symbology. However, many barcodes do not fit into either of these categories, particularly those most recently developed.

\section{Stacked Symbologies}

A stacked symbology is the most primitive of all possible two-dimensional schemes, and perhaps the most intuitive when starting with a linear barcode system. Several linear barcodes of a given symbology are truncated and then layered vertically to create the stacked symbology. A much higher data-density than the linear code is achieved at the price of less vertical redundancy.

Stacked barcodes were optimized to be read using a laser scanner in which the laser beam is swept several times horizontally as it makes its way down the barcode vertically. Certain symbologies, such as Codablock, allow the barcode to be read by a linear barcode 


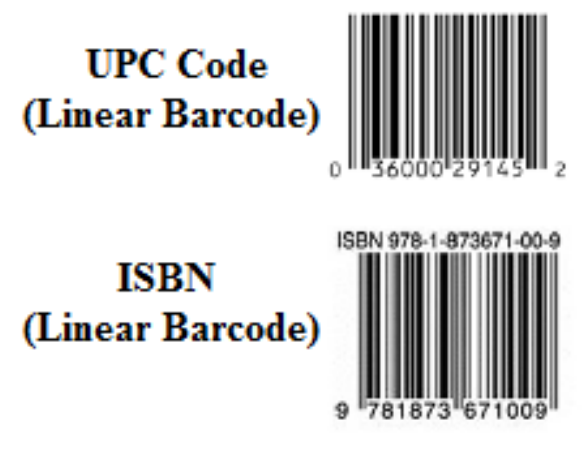

PDF417

Code 49

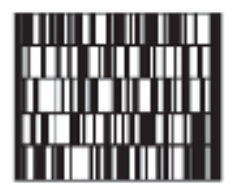

Code 16K

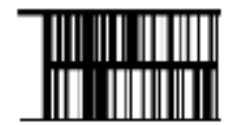

Codablock

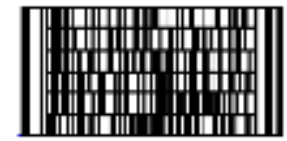

UltraCode

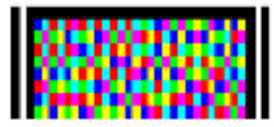

Maxicode

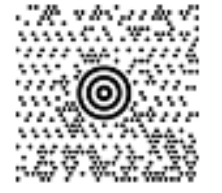

Figure 2.1: List of Common Barcode Symbologies [1, 2, 3, 4] 
reader (a standard moving beam laser) with very little modification. CCD imagers are also used to read stacked-symbology barcodes. The scanning requirements and constraints are specific to the code symbology.

Many stacked-symbology barcodes continue to be used in various industries, such as Code 16K and Codablock (Figure 2.1) in the health care industry, and PDF 417 (Figure 2.1) in transportation, personal identification and inventory management domains $[1,19]$. However, despite its successes, more efficient encoding methods have made the stacking technique obsolete.

\section{Matrix Symbologies}

Matrix codes encode data through the positioning of equal-dimension spots within a matrix (dark spots on a light surface). The symbology usually includes patterns that indicate the orientation of the barcode, and often convey the size and printing density of the barcode as well. CCD imagers and camera capture devices are used to scan matrix barcodes.

There are several matrix symbologies in the public domain, as well as many proprietary ones. United Parcel Service (UPS) developed its own well-known matrix symbology, Maxicode (or UPSCode), in 1992 to label and track packages. Maxicode is made up of interlocking hexagons and includes a central bull's-eye marking to aid in acquisition (see Figure 2.1). The code requires a very high resolution printer, but can be read by a CCD scanner or camera when even up to $25 \%$ of the symbol is destroyed.

Matrix symbologies are often created with specific practical constraints in mind. For instance, the Aztec Code was designed to be easily printed and decoded, the QR Code was designed for rapid reading using CCD array cameras, and the Data Matrix was created to achieve a very high data-density (see Figures 2.3 and 2.4). As a result, matrix symbologies tend to be more application-specific than stacked symbologies.

Matrix symbologies generally offer many advantages over stacked symbologies, primarily because they use space more efficiently and scatter redundancy to increase robustness. However, the drawbacks of matrix codes are prompting the creation of symbologies that diverge from the traditional matrix model. Although most new symbologies do not fit either the stacked or matrix definitions, many do resemble matrix codes in some way, and often employ many of the same concepts. 


\section{Other Two-Dimensional Symbologies}

Several 2-D barcodes cannot be categorized as having either a stacked or a matrix symbology. DataGlyphs, for instance, are made up of forward and backward slashes (/ and $\backslash$ ), representing binary ' 0 's and ' 1 's respectively (Figures 2.6 and 2.7). INTACTA.CODE is a propriety code that converts binary files and software into a very high-density machine-readable symbol of scattered dots (Figure 2.2). Advancements in computer imaging techniques and devices have made coloured barcodes more feasible for a variety of applications. As a result, some barcodes use colour to increase data-density, such as the HCCB (High Capacity Color Barcode, seen in Figure 2.5), the Ultracode (Figure 2.1), and the HueCode.

The newest barcode symbologies offer more unique features and are difficult to categorize. Developments continue as demands on current barcode systems grow and new barcode applications emerge. There are advantages and drawbacks to each barcode design, and symbologies are usually designed for specific requirements and constraints. There has been significant progress in recent years, and development is expected to continue in the barcode symbology domain [9].

\subsection{Specifics of Notable Two-Dimensional Barcodes}

It is worth examining some of the more notable 2-D barcodes. Whether they have gained popularity in certain fields of use, or offer unique features to the user, they can give insight into what has already been accomplished as well as potential improvements for the future. Six significant 2-D symbologies are briefly described and then compared.

\subsubsection{INTACTA.CODE}

INTACTA.CODE is a proprietary code developed by INTACTA Technologies, Inc. that converts binary files into a graphical representation, thus securing information over electronic media and in printed form. The development of INTACTA.CODE was initiated by the defence industry, where privacy is of critical importance. In addition to security, INTACTA Technologies places emphasis on bandwidth efficiency (to efficiently increase data-density) and error-correction abilities, while being easy and practical to adopt for various applications. 


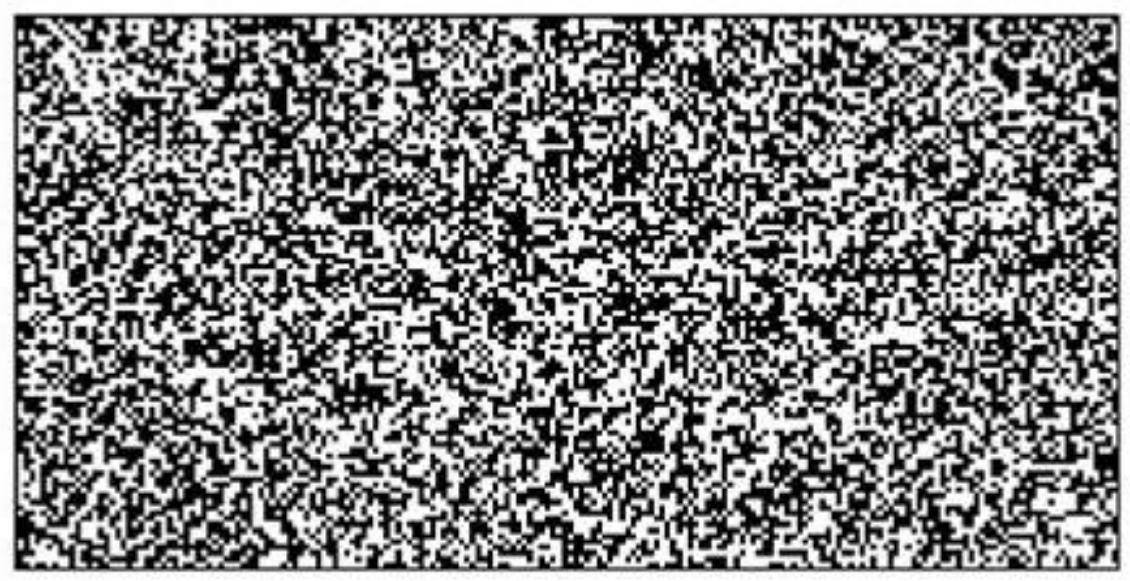

Figure 2.2: INTACTA.CODE Symbol [20]

INTACTA.CODE looks like a random arrangement of dots (Figure 2.2). Each byte of information contained in the symbol is represented by a small pattern of black and white dots. Prior to encoding, the data is compressed and encrypted by the INTACTA software, with a high level of flexibility left to the user. The barcode can then be created in a printable or a digital format. Also, because the code is not based on a fixed architecture, it can be formed into any desired continuous shape.

INTACTA.CODE can be read by any off-the-shelf scanner and is decoded by proprietary software. The data-density depends on the printing and scanning resolutions. INTACTA.CODE achieves 1000 bytes $/$ inch $^{2}\left(1240\right.$ bits $\left./ \mathrm{cm}^{2}\right)$ at $300 \mathrm{dpi}$ resolution and 3800 bytes/inch ${ }^{2}\left(4712\right.$ bits $\left./ \mathrm{cm}^{2}\right)$ at $600 \mathrm{dpi}$ resolution, assuming equal printing and scanning resolutions. Intacta Technologies has indicated that a colour INTACTA.CODE is also possible, but such a symbol has yet to be presented publicly. See [20, 21, 22] for more information.

\subsubsection{Data Matrix}

As its name suggests, Data Matrix employs a matrix symbology. It was patented in 1991 and achieves a high data-density compared to other barcodes of its time. The Data Matrix symbol is also variable in size, giving it a significant advantage over several fixed-architecture barcode symbols. A Data Matrix symbol consists of a matrix of equal-sized squares, as illustrated in Figure 2.3. It is read by CCD camera / scanner.

The perimeter of the Data Matrix symbol indicates the density of data contained within 


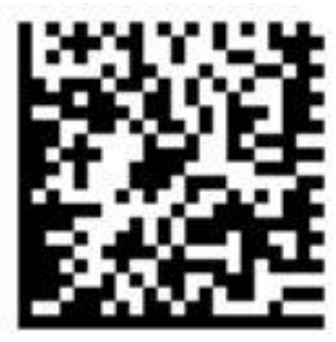

Figure 2.3: Data Matrix Symbol [23]

the matrix. The product of the number of light squares and dark squares of the first and second sides correspond to the number of bits of information contained in the symbol, while the solid dark lines on the third and fourth sides indicate the height, length and area of the symbol. Because of the information contained in the perimeter, the Data Matrix code can be scanned from any orientation, as well as at an angle using a camera capture device.

Instead of representing individual bits by individual light/dark squares, the Data Matrix symbology compresses the input data by defining a maximum range of characters that may appear in an input string, and removes redundancy to reduce the number of squares required to represent that string. For this reason, the compression process depends on the type of input character anticipated.

Robustness is increased by scattering redundant data throughout the symbol. This is accomplished by randomly positioning redundant cells (squares in the symbol) as far as possible from the root cell encoded. The user can specify the redundancy level (up to 400\%). Convolutional coding was originally applied, but Reed-Solomon error correction algorithms are now used, allowing the Data Matrix barcode to be decoded when up to $60 \%$ of the symbol is damaged.

The data-density achieved by a Data Matrix symbol depends on the size of the symbol, the size of the cells (which will depend on the scanning resolution), and the amount of redundancy included. In theory, the symbol can store 500 characters in a square milli-inch. This is, of course, impractical when taking printing and scanning resolutions into account. More realistically, using $600 \mathrm{dpi}$ and 4 dots per module, a data-density of approximately $1838 \mathrm{bits} / \mathrm{cm}^{2}$ is achieved (see Appendix A).

The Data Matrix code has been in the public domain for almost a decade and continues to be widely used. Because the barcode is well-suited to the labelling of small items, it is 


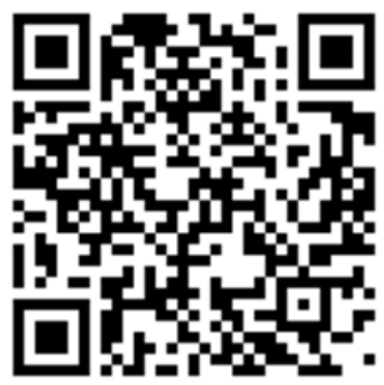

Figure 2.4: QR Code Symbol [26]

used in the electronics industry to mark integrated circuits and printed circuit boards. A more recent function for the code is to link paper to the web via cell phone cameras. The robustness, flexibility, and relatively high data-density offered by Data Matrix makes it an appealing candidate for a variety of applications. More information on the Data Matrix code can be found in $[24,25]$.

\subsubsection{QR Code}

The Quick Response (QR) Code was developed by the Japanese company Denso Wave and was approved as ISO international standard in June, 2000 [23]. The code is in the public domain and is well-known, particularly in Japan. As its name may suggest, the QR Code was designed to be read rapidly with CCD array cameras and image processing technology. As a result, the QR Code can be easily interpreted using low-resolution scanning and with low decoding complexity compared to other 2-D barcodes.

Like the Data Matrix, the QR Code employs a square matrix symbology. Light and dark square cells represent binary '0's and '1's respectively. QR symbols have a distinct appearance due to their position detection patterns in three corners, which look like square bull's-eyes (see Figure 2.4). The position detection patterns allow for quick recognition by the image processor and indicate the symbol's orientation with the help of a scanning line which runs through the centre of the symbol.

QR Codes have forty symbol versions which differ in module configuration and number (modules are the square cells in the symbol matrix). QR Versions 1 through 40 progress from $21 \times 21$ modules to $177 \times 177$ modules respectively, with a $4 \times 4$ module increase per version. Each version offers a unique data capacity which depends the type of character 
and the level of error-correction coding (Reed-Solomon coding is employed to increase robustness). A typical symbol offers an error-correction rate of approximately $15 \%$ (where rate is considered to be the ratio of number of correctable code words to total code words). However, the level of error correction required will depend on the environment of usage, and four different levels are possible (approximately 7\%, 15\%, 25\%, and 30\%) [27].

A single QR Code symbol can be divided into up to sixteen symbols, and several symbols can conversely be reconstructed into a single data symbol. This feature gives the user some spatial flexibility and allows for printing in a narrow area.

Like most 2-D codes, the QR Code data-density depends on several factors. The module size depends heavily on the printing and scanning resolutions. For example, if a QR Code symbol is printed with a $600 \mathrm{dpi}$, 4-dot printer, the module size is $0.17 \mathrm{~mm}$ and will therefore require a scanner resolution of less than $0.17 \mathrm{~mm}$. An example of QR Code data-density is approximately $330 \mathrm{bits} / \mathrm{cm}^{2}$ using a Version 3 symbol with a 15\% error-correction rate, printed with 400 dpi resolution [27].

The easy recognition and low decoding complexity of QR Codes make them a practical barcode for cell phone usage, where processing capacity is limited. QR codes are currently being displayed on mobile phone screens for the purpose of ticketless concert entry and cashless vending machine purchases. Many cell phones in Japan are equipped with QR decoders to read barcode advertisements. These applications are creating a rapidly expanding new market for QR codes. See [27, 28] for more information.

\subsubsection{HCCB (High Capacity Color Barcode)}

The HCCB (High Capacity Color Barcode) is one of the newest barcode symbologies to be introduced. It is a proprietary code by Microsoft Corp. that is awaiting a patent to be issued in the United States. By using colour, the HCCB achieves a high data density and has been designed with consumer cell phone applications in mind.

Triangular symbols are arranged compactly throughout the symbol, as in Figure 2.5. The triangles are separated by white spacing that serves to reduce aliasing effects and other distortions. A similar symbol structure using geometric shapes other than triangles is also possible for the HCCB, but triangles are currently being used because they are more efficiently packed. 

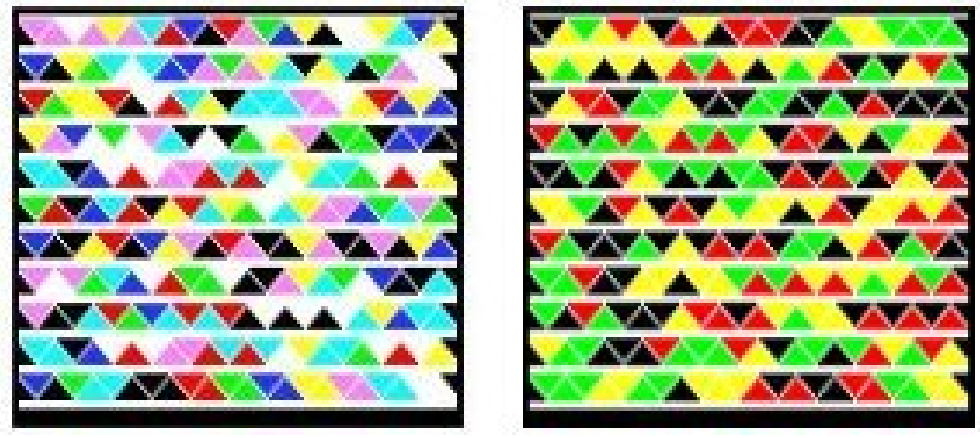

Figure 2.5: HCCB Symbols [29]

The HCCB includes CRC (Cyclic Redundancy Check) error-detection and Reed-Solomon coding for error-correction. A reference colour palette is included in the HCCB symbol to allow for a weighted adjustment that reflects or compensates for variations in dynamic range perceived by the camera/scanning device. Should the reference palette on the barcode symbol be damaged, the necessary adjustments can still be made based on history or previously scanned palette colour values (Microsoft claims this technique has been proven to be empirically reliable [25]).

Data-density varies in relation to the colour scheme chosen. Microsoft offers its HCCB in black and white, with four different colours, and with eight different colours. Other schemes, such as grayscale, are also possible and may be available in the future. Using the eight-colour design at $600 \mathrm{dpi}$ resolution, a data-density of 2000 bytes $/$ inch $^{2}$ can be achieved (2480 bits $\left./ \mathrm{cm}^{2}\right)$.

The HHCB can be read in a variety of ways, including using a flat-bed scanner, a business card reader scanner, a digital camera, a video camera, or a Web cam. Microsoft is promoting potential cell phone applications, highlighting that the HCCB outperforms QR Code and Data Matrix formats for cell phone scanning and decoding.

Microsoft Corp. and the International Standard Audiovisual Number International Agency (ISAN-IA) announced an agreement whereby ISAN-IA has licensed the HCCB to assist in the identification of commercial audiovisual works such as motion pictures, video games, broadcasts, digital video recordings and other media. The barcode is expected to start appearing on DVDs and Xbox games in the near future $[29,30]$. More information can be found at $[6,29,30,31]$. 


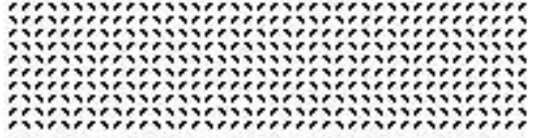

Figure 2.6: Data Glyph Symbol [32]

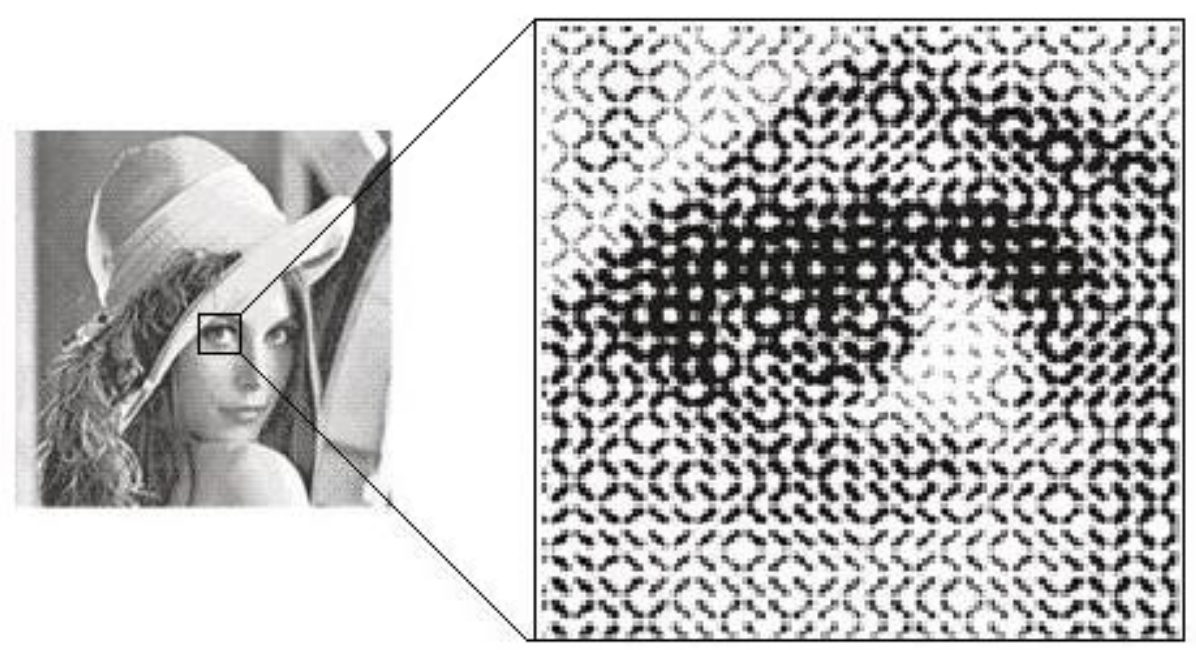

Figure 2.7: Data Glyph symbol using data toning to resemble a photo [32]

\subsubsection{Data Glyphs}

DataGlyphs are a notable 2-D symbology because of their flexible shape and size, and their unique, recognizable ability to blend into pictures (see Figures 2.6 and 2.7). They are also suitable for curved surfaces, unlike most other barcodes.

DataGlyphs were invented by the Palo Alto Research Center (PARC), a part of Xerox Research, to embed computer-readable data on paper for document management systems. Although they were developed in the early 1990's, DataGlyphs only gained popularity almost a decade later. The PARC software patents were licensed to Microglyph Technology who went on to develop additional proprietary codes for the labelling of parts in the manufacturing industry, and to enable the embedding of computer-readable data on surfaces such as plastic, glass, or metal [28]. Basic DataGlyphs are a pattern of forward and backward slashes representing binary ' 1 's and '0's. By combining different types of DataGlyphs, such as those of differing fonts and colours, one can expand the encoding possibilities.

A DataGlyph symbol contains an embedded synchronization lattice. The lattice is a 
repetitive fixed pattern of glyphs that indicates the symbol boundaries and serves as a clocking track to improve the reliability of reading. Groups of glyphs, representing bytes of data, are laid down within this lattice frame. The data is sectioned into blocks of a few dozen bytes each. Error-correction coding is added to each block at an appropriate level for the particular application (faxing, for instance, requires a substantial amount of error-correction coding). As a final step, the bytes of data are randomly dispersed across the entire area of the DataGlyph symbol. The built-in error correction code and data randomization give DataGlyphs a high level of reliability [32].

DataGlyph symbols have adjustable size, shape, and colour. Their data-density, like other barcodes, depends on the printing/scanning resolution, the amount of error-correction coding, the data compression used, and the amount of overhead imposed by the synchronization lattice (a fixed proportion of the data area). DataGlyphs boast a relatively high data-density. For instance, at $27 \%$ error correction and 400 dpi resolution, a DataGlyph symbol can achieve 500 bytes/inch ${ }^{2}\left(620 \mathrm{bits} / \mathrm{cm}^{2}\right)$.

DataGlyphs are read using two-dimensional imaging devices such as scanners or cameras, and can be captured at any orientation. Xerox offers many coding possibilities, such as Yellow DataGlyphs to embed data invisibly in a printed document and Data Toning to aesthetically modulate the glyphs to resemble a picture (Figure 2.7). DataGlyphs have been used in a wide range of Xerox products, as well as in other markets, such as financial services, software, government, health care, and pharmaceuticals. More information can be obtained from [32].

\subsubsection{Granite Codes}

The Granite Code, seen in Figure 2.8, is a new two-dimensional symbology developed at the University of Toronto [33]. Although the symbology has no public recognition, it is notable because of its unique modulation techniques. Granite Codes achieve a very high data-density by using the spatial bandwidth of the channel efficiently. Granite Codes are not the only barcode to employ such techniques, but they are worth examining in closer detail.

Granite Codes are based on Spatial Discrete Multi-Tone Modulation (SDMT) and exploit the low-pass characteristics of a camera lens channel. Digital cameras, like human eyes, can 


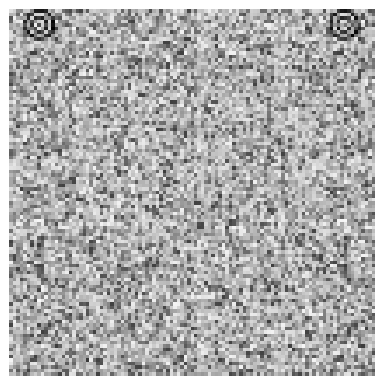

Figure 2.8: Granite Code by Gupta and Kschischang [33]

be modelled as having an optical transfer function that imposes a high gain at low frequencies and attenuation at high frequencies. The Granite Code symbology takes advantage of this low-pass attribute of digital cameras by employing orthogonal frequency-division multiplexing (OFDM) and a process called digital half-toning with an error-diffusion algorithm.

The coding process for Granite Codes consists of adding CRC bits to the data, encoding with a Repeat Accumulate (RA) Code, and mapping and modulating symbols into frequency bins. Once this is done, the barcode is transformed to the space domain, which produces a grayscale image. The half-toning process then converts the image to binary representation. Finally, image registration marks, called fiducials, are added so that the barcode can be detected properly, and orientation can be easily determined.

The digital half-toning employed for Granite Codes relies on an error-diffusion algorithm which shapes the quantization noise to higher frequency bins by using a noise-shaping feedback quantizer (see $[33,34]$ for more information). Because the digital camera operates as a low-pass filter on the image, the noise is filtered out. A dynamic range compression algorithm is applied to the data in order to carry out a Water-Pouring process that judiciously allocates power into frequency bins that are within the Nyquist band of the receiver. This clever coding technique exploits the nature of the channel, thus achieving much greater bandwidth efficiency than popular barcodes available on the market. This translates directly to a higher data-density and allows the Granite Code symbol to pack more information into a smaller spatial area.

Granite Codes are flexible in shape and size, and were designed to be read by a digital camera (any two-dimensional imaging device will work). The image of the barcode must be captured at an orthographic angle, and the code has the disadvantage of being sensitive 


\begin{tabular}{lc} 
Barcode Type & $\begin{array}{c}\text { Typical Data Density } \\
\left(\text { bits } / \mathrm{cm}^{2}\right)\end{array}$ \\
\hline \hline QR Code & 1063 \\
DataGlyphs & 1428 \\
Data Matrix & 1838 \\
HCCB & 2480 \\
INTACTA.CODE & 4712 \\
\hline
\end{tabular}

Table 2.1: Density of Notable Barcode Symbologies at 600 dpi. Note that some parameters may not be constant (for instance, error-correction \%). Table values demonstrate a general data-density comparison and may not reflect exact differences between symbologies. See Appendix A for more information.

to nonlinearities. To compensate, Gupta suggests pre-distortion techniques in his thesis.

A typical data-density achieved by the Granite Code is presented by Gupta in comparison to other popular symbologies. At a module size of $3.5 \times 3.5$ camera pixels, the Granite Code symbol boasts a $1200 \mathrm{bits} / \mathrm{cm}^{2}$ data-density versus $100 \mathrm{bits} / \mathrm{cm}^{2}, 70 \mathrm{bits} / \mathrm{cm}^{2}$, and 50 bits $/ \mathrm{cm}^{2}$ for the Data Matrix, PDF417, and QR Code symbols respectively [33]. However, Granite Codes do not offer a significant data-density advantage over other spatially bandwidth-efficient symbologies, such as INTACTA.CODE (4712 bits $/ \mathrm{cm}^{2}$ at $600 \mathrm{dpi}$ ) or SECURITY2image, S2i (4650 bits $/ \mathrm{cm}^{2}$ at $\left.600 \mathrm{dpi}\right)[20,35]$.

\subsection{Symbology Comparison}

Table 1 demonstrates the data-density achieved by the aforementioned symbologies at a printing resolution of $600 \mathrm{dpi}$ (this assumes equal scanning resolution). The corresponding calculations can be found in Appendix A.

Although data-density is a key measure of a symbology's value, there are other important factors taken into account. Robustness, size and shape flexibility, decoding complexity, and symbol sensitivities to scanning errors (such as skewing) are crucial components of a comprehensive code comparison.

Comparisons between symbologies depend heavily upon the application. Most indus- 
tries that currently use barcode systems are unlikely to adopt an entirely new code symbology, even if it asserts a significant upgrade, because of the immense investment involved with a new system, coupled with a lack of need. It is therefore worth comparing the symbologies with new applications mind.

Although INTACTA.CODE and similar symbologies, such as Granite Code and S2i Code, have the highest data-densities, they impose a greater decoding complexity than the HCCB code, for instance, and are more sensitive to nonlinearities. INTACTA.CODE was designed to interface paper with the digital world through the use of flat-bed scanners and high processing-power devices, such as computers, and achieves this objective well. However, it may be cumbersome to adopt the INTACTA.CODE for cell phone applications. The QR Code has significant advantages in this area, as does the more data-dense HCCB symbol. For cell phone specific usage, further investigation is required to make an adequate evaluation.

When choosing an appropriate 2-D barcode, it is necessary to establish the level of errorcorrection ability required for the given application, as well as the scanning and decoding constraints. If there are also important aesthetic considerations, some symbols may not be appropriate. Once these requirements are shown to be met by a set of codes for the given application, one can choose the most data-dense symbol in the set. However, given the inherent limitations of current symbologies, it may be worth designing a new barcode that is superior for the specific application. That way, resources can be allocated more efficiently to accommodate the unique constraints.

\subsection{Research Phases}

A barcode design that is lacking from the array of symbologies is one that optimizes optical communication over a camera phone channel. The ultimate goal of this thesis was to design a symbology that is suitable for cell phone camera applications. In order to do so, the following research phases were laid out:

\section{Develop a Channel Model}

2. Create a Simulation Environment 
3. Justify the Channel Model with Trials and Measurements

4. Design Stage:

(a) Conceptual Design

(b) Image Processing Techniques

(c) Coding Techniques

The channel model is crucial in the design process of communication systems. Often, channel models are well established and require only slight modifications for the application of interest. However, for the wireless optical channel with a cell phone camera receiver, no such model exists. Research into the sources of noise and distortion applied to the transmitted barcode must be conducted. The resultant mathematical representation of the channel should then be justified through experimentation with a camera phone.

Only once a good understanding of the channel has been established can coding and image processing techniques succeed. It is possible that an existent barcode, such as the granite code, may be optimal for camera phone applications. However, it is likely that a superior symbology can be developed with coding and image processing techniques that help overcome the unique channel constraints. 


\section{Chapter 3}

\section{Mathematical Channel Model}

In order to design an appropriate barcode for cell phone camera applications, it is crucial that a reasonable channel model be established. The symbologies developed thus far were not designed to overcome any channel-specific parameters, and therefore, fall short in cell phone camera applications. If the sources of distortion and noise imposed on the barcode data by the wireless optical channel specific to cell phones could be identified and modelled appropriately, effective coding and image processing techniques can be employed to overcome channel hindrances.

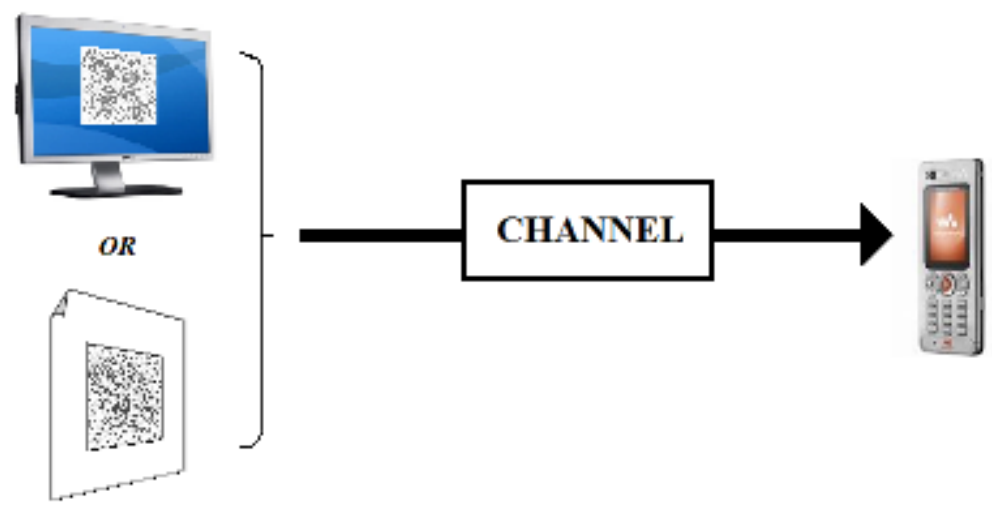

Figure 3.1: Basic Channel Pictorial Diagram

The basic channel model can be described by the block diagram in Figure 3.1. The channel can be perceived as a black box that accepts a barcode image as an input, and then outputs a distorted and noisy version of this image. A more descriptive model of the process should be developed, with the sources of distortion and noise within the black box 
identified and modelled mathematically.

The overall channel model, where $g(i, j)$ denotes the output image corresponding to input barcode $f(x, y)$ (note the change in coordinates), can be described mathematically by

$$
g(i, j)=J\{C[D(v * W(f(x, y)))]+n(i, j)\},
$$

where the parameters are as follows:

$W$ denotes a warping function representing the geometrical transformation applied to the barcode image corresponding to camera orientation. W maps $f(x, y)$ to $f^{w}(i, j)$.

$v$ denotes the point spread function (PSF) of the channel. (* denotes convolution)

$D$ denotes the CMOS sensor function that distorts the image. It can be broken down into two parts: a sensor PSF and a sampling function as well as a nonlinear response to light intensity with colour cross-talk.

$C$ denotes the digital signal processing applied by the camera software (i.e. gamma correction, automatic white balance, etc.)

$n$ denotes additive white Gaussian noise (AWGN).

$J$ denotes the automatic JPEG compression function of the cell phone camera.

To understand the physical parameters and develop an appropriate mathematical model of the channel, the distortion and noise contributors identified in Equation 3.1 should be examined independently.

\subsection{Image Warping}

Imaging geometry is critical in deriving a camera model. All transformations can be expressed in a three-dimensional Cartesian coordinates system, where every point is assigned coordinates $(X, Y, Z)$. Because an image is two-dimensional, it will represented using lowercase $(x, y)$, the pixel coordinates. The barcode image can then be viewed geometrically as a plane in the three-dimensional Cartesian system.

In general, cameras map three-dimensional points to a two-dimensional plane. This transformation of the three-dimensional world-view to a two-dimensional photograph is a 

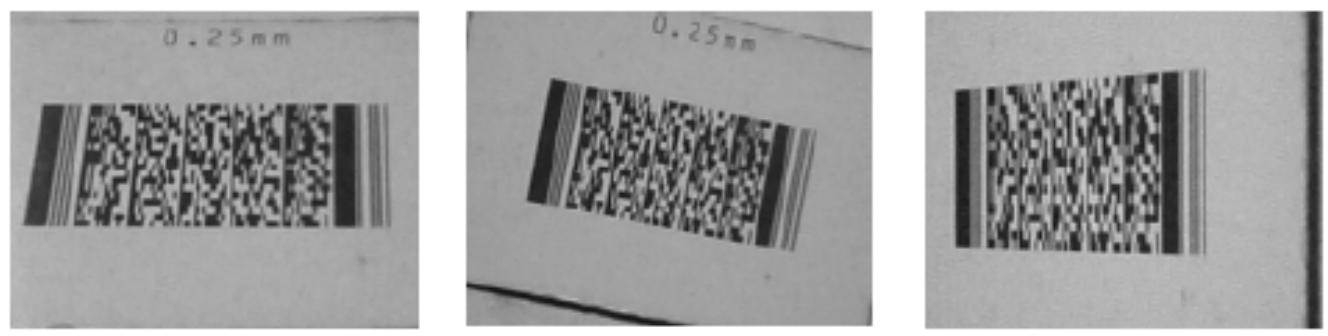

Figure 3.2: Barcode Skewing (Rotations about the $\mathrm{x}, \mathrm{z}$, and $\mathrm{y}$ axes respectively from left to right) [36]

perspective projection. Unlike orthographic projections, perspective projections are nonlinear, and approximate the way a human eye would perceive the scene. That is, objects farther away appear smaller than objects in the near-view.

For the case of a barcode image being photographed, a two-dimensional object in the three-dimensional world is being represented in the picture. Ideally, the camera is positioned orthogonally such that the camera axes are aligned with the barcode axes. However, this is unlikely to occur in practice, particularly with the use of cell phone cameras. There is likely to be an angle of rotation of the camera axes with respect to the barcode axes, causing the barcode image to be skewed. Figure 3.2 displays examples of skewing (left and right photos).

The camera's position, orientation, and field of view control the behaviour of the projection transformation applied to the barcode. The projective transformation applied to a three-dimensional "'World"' point $\left(a_{x}, a_{y}, a_{z}\right)$ can be described by the concatenation of three transformations: 1) translation of the World origin to the image plane origin, 2) rotation of the point according to camera orientation, 3) projection of the point onto the image plane. The overall result is a mapping from the three-dimensional point, $\left(a_{x}, a_{y}, a_{z}\right)$, to a two-dimensional point, $\left(b_{x}, b_{y}\right)$, as seen in Figure 3.3. The process is described mathematically by

$$
\left(b_{x}, b_{y}\right)=\left(d_{x}-e_{x}, d_{y}-e_{y}\right) \frac{e_{z}}{d_{z}}
$$


where

$$
\left[\begin{array}{l}
d_{x} \\
d_{y} \\
d_{z}
\end{array}\right]=R \cdot\left(\left[\begin{array}{l}
a_{x} \\
a_{y} \\
a_{z}
\end{array}\right]-\left[\begin{array}{l}
c_{x} \\
c_{y} \\
c_{z}
\end{array}\right]\right)
$$

and $R$ is the rotation matrix. Note that $\left(e_{x}, e_{y}, e_{z}\right)$ is a point of convergence behind the image plane as seen in Figure 3.3.

The rotation of a point about the $z, x$, and $y$ axes are achieved using transformation matrices $R_{\alpha}, R_{\beta}$, and $R_{\lambda}$ respectively, where $\alpha, \beta$, and $\lambda$ denote the respective angles of rotation. Homogeneous coordinates allow projective transformations to be represented in matrix form. In homogeneous coordinate representation, the matrices are expressed as

$$
\begin{aligned}
& R_{\alpha}=\left[\begin{array}{cccc}
\cos \alpha & -\sin \alpha & 0 & 0 \\
\sin \alpha & \cos \alpha & 0 & 0 \\
0 & 0 & 1 & 0 \\
0 & 0 & 0 & 1
\end{array}\right] \\
& R_{\beta}=\left[\begin{array}{cccc}
1 & 0 & 0 & 0 \\
0 & \cos \beta & -\sin \beta & 0 \\
0 & \sin \beta & \cos \beta & 0 \\
0 & 0 & 0 & 1
\end{array}\right] \\
& R_{\lambda}=\left[\begin{array}{cccc}
\cos \lambda & 0 & -\sin \lambda & 0 \\
0 & 1 & 0 & 0 \\
\sin \lambda & 0 & \cos \lambda & 0 \\
0 & 0 & 0 & 1
\end{array}\right] .
\end{aligned}
$$

These three affine transformations can be concatenated together to form $R=R_{\beta} \cdot R_{\lambda} \cdot R_{\alpha}$ 


$$
=\left[\begin{array}{cccc}
\cos \lambda \cdot \cos \alpha & \cos \lambda \cdot \sin \alpha & \sin \lambda & 0 \\
-\cos \beta \cdot \sin \alpha- & \cos \beta \cdot \cos \alpha- & \sin \beta \cdot \cos \lambda & 0 \\
\sin \beta \cdot \sin \lambda \cdot \cos \alpha & \sin \beta \cdot \sin \lambda \cdot \sin \alpha & & \\
\sin \beta \cdot \sin \alpha- & -\sin \beta \cdot \cos \alpha- & \cos \beta \cdot \cos \lambda & 0 \\
\cos \alpha \cdot \cos \beta & \sin \alpha \cdot \cos \beta \cdot \sin \lambda & & \\
0 & 0 & 0 & 1
\end{array}\right] .
$$

Note that under this ordering, the rotation about the z-axis is applied first. This does not skew the image, but simply rotates it counter-clockwise as in the second example of Figure 3.2. The $y$-axis tilt follows next and the $x$-axis tilt is done last. For more information on homogeneous coordinates, see [37].

When rotating the barcode plane in three-dimensions, the transformation is linear and invertible. However, unlike rotational transformations, perspective transformations are not invertible. The perspective transformation described by Equation 3.2 maps a threedimensional point to a two-dimensional point in the image plane - a process that is clearly not a bijection. Figure 3.3 illustrates a perspective projection onto an image plane, where the barcode image is skewed with respect to the camera axis. For more information on projections and geometric transformations, see [38, 39].

It is often more intuitive to separate the rotation transformation from spatial warping. To apply a simple rotation to the barcode image, each $(x, y)$ pixel is multiplied by

$$
R_{\alpha}^{2 D}=\left[\begin{array}{ccc}
\cos \alpha & -\sin \alpha & 0 \\
\sin \alpha & \cos \alpha & 0 \\
0 & 0 & 1
\end{array}\right] .
$$

Once this is done, the actual warping can be examined separately.

In photography, the horizontal displacement camera angle is referred to the camera pan. This corresponds to a rotation about the y-axis in the previous model. The vertical angle ( $\mathrm{x}$-axis tilt) is referred to as the camera tilt. For the following warping description, let $\beta$ represent the pan angle and $\lambda$ represent the tilt angle. 


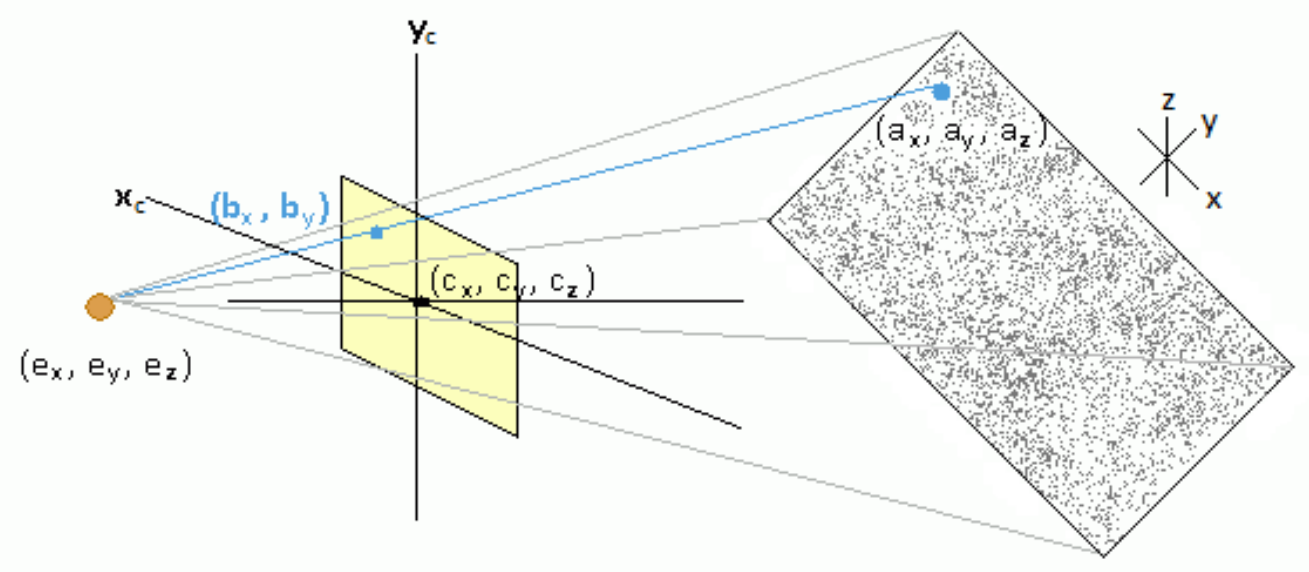

Figure 3.3: Perspective Projection: Camera Axes and Barcode Axes not Aligned

Because the image plane in a camera is a distance $f$ from the lens, where $f$ is the focal length of the lens, it is known that the distance from point $\vec{c}$ to point $\vec{e}$ in Figure 3.3 is $f$. Therefore, the projective transformation can be reduced to

$$
\left(b_{x}, b_{y}\right)=\left(\frac{f \cdot a_{x}}{f-a_{z}}, \frac{f \cdot a_{y}}{f-a_{z}}\right) .
$$

For the case where the camera is not positioned at the center of the world origin, the same procedure of translation, followed by rotation and projection, must be applied. The result can be described by

$$
\vec{b}=P \cdot R_{\beta} R_{\lambda} \cdot T \vec{a},
$$

where $\mathrm{P}$ is the projection matrix, the rotation matrices are as defined previously, and $\mathrm{T}$ is the translation matrix given by

$$
T=\left[\begin{array}{cccc}
1 & 0 & 0 & -c_{x} \\
0 & 1 & 0 & -c_{y} \\
0 & 0 & 1 & -c_{z} \\
0 & 0 & 0 & 1
\end{array}\right]
$$

Overall, this gives the final result described by 


$$
b_{x}=\frac{f\left[\left(a_{x}-c_{x}\right) \cos (\lambda)-\left(a_{y}-c_{y}\right) \sin (\lambda) \sin (\beta)-\left(a_{z}-c_{z}\right) \sin (\lambda) \cos (\beta)\right]}{f-\left(a_{x}-c_{x}\right) \sin (\lambda)-\left(a_{y}-c_{y}\right) \cos (\lambda) \sin (\beta)-\left(a_{z}-c_{z}\right) \cos (\lambda) \cos (\beta)}
$$

and

$$
b_{y}=\frac{f\left[\left(a_{y}-c_{y}\right) \cos (\beta)-\left(a_{z}-c_{z}\right) \sin (\beta)\right]}{f-\left(a_{x}-c_{x}\right) \sin (\lambda)-\left(a_{y}-c_{y}\right) \cos (\lambda) \sin (\beta)-\left(a_{z}-c_{z}\right) \cos (\lambda) \cos (\beta)}
$$

for the $\mathrm{x}$ and $\mathrm{y}$ image coordinates respectively.

To apply the transformation to an entire $M \times N$ barcode image, the 3-D image coordinates of the barcode plane are all mapped to new 2-D coordinates by these two equations. To facilitate the translation, a $3 \times(M \cdot N)$ matrix of coordinates can be created containing all image points and Equation 3.6 can be applied to the entire matrix, as opposed to vector $\vec{a}$. The result is a $2 \times(M \cdot N)$ matrix containing the transformed image points.

In summary, camera orientation can skew the barcode. The transformation is a perspective projection, described by Equations 3.8 and 3.9, and does not, in general, preserve shape (circles project to ellipses, parallel lines become unequally foreshortened, etc.). For this reason, the geometry of the barcode will not be preserved unless it is photographed orthographically, which cannot be guaranteed. A reasonable degree of skewing should be anticipated and a barcode solution for cell phone cameras must successfully decode under such conditions.

\subsection{Point Spread Function (PSF)}

The PSF is the channel impulse response, describing the response of an imaging system to a point source. The wireless optical communication channel can be described as a twodimensional low-pass filter. To gain an understanding of this low-pass characteristic and the relationships between optics and communication theory, an overview of Fourier Optics and the underlying mathematical Fourier analysis is required.

\subsubsection{Diffraction}

Fourier Optics is based on the plane wave spectrum representation of optical fields, which is analogous to Fourier analysis of electrical signals. Plane waves are described in the illustration of ray bundles in Figure 3.4. 

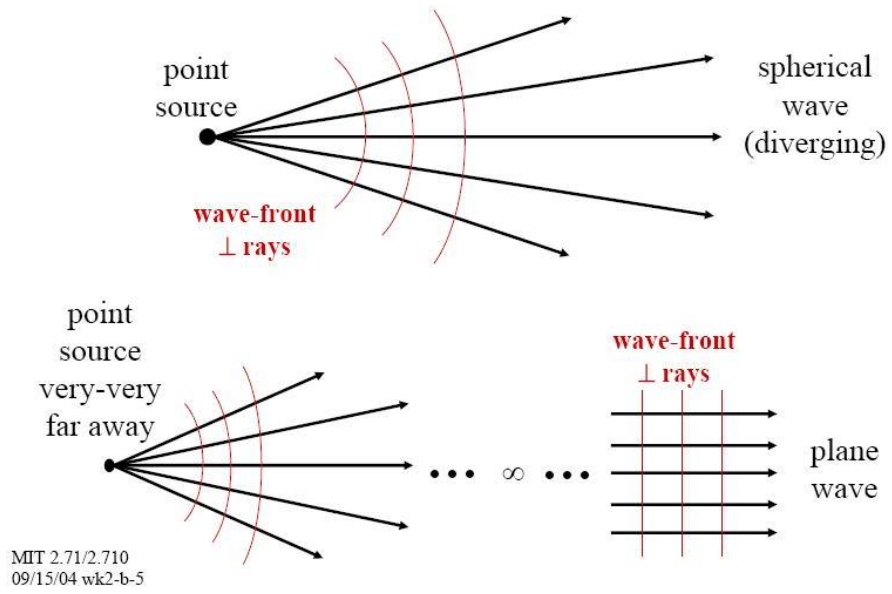

Figure 3.4: Spherical versus Planar Wave Patterns [40]

The bandwidth in electrical signals relates to the difference between the highest and lowest frequencies present in the spectrum of the signal. For optical systems, spatial bandwidth is a measure of how far a plane wave is tilted away from the optic axis (see Figure 3.5). It takes more frequency bandwidth to produce a short pulse in an electrical circuit, and more spatial bandwidth to produce a sharp spot in an optical system.

A better understanding of these concepts can be gained by considering a scenario where monochromatic light is emitted through a finite aperture in an infinite screen, and observed on a plane parallel to the screen some distance away. An examination of how the light is diffracted leads to the fundamental concepts of Fourier Optics.

Fresnel diffraction describes a process that occurs when an optical wave passes through a finite aperture and diffracts in the near field, which is why it is often referred to as nearfield diffraction. The observed diffraction patterns differ in size and shape relative to the distance from the aperture.

Referring to Figure 3.6, if the distance between the aperture and observation plane is increased such that $\overrightarrow{r_{01}}$ becomes approximately parallel to the z-axis in Figure 3.6, the optical waves are essentially planar. For such distances, Fraunhofer diffraction, or far-field diffraction, is said to occur. 


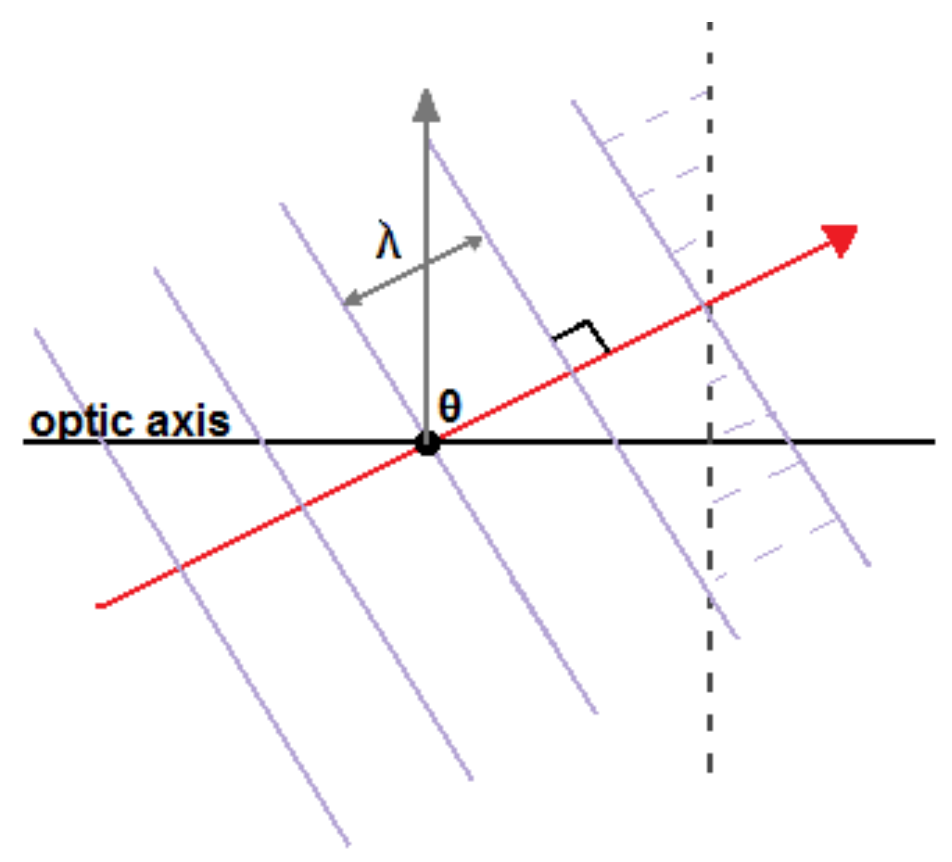

Figure 3.5: Plane Wave Tilt from Optic Axis (after [40])

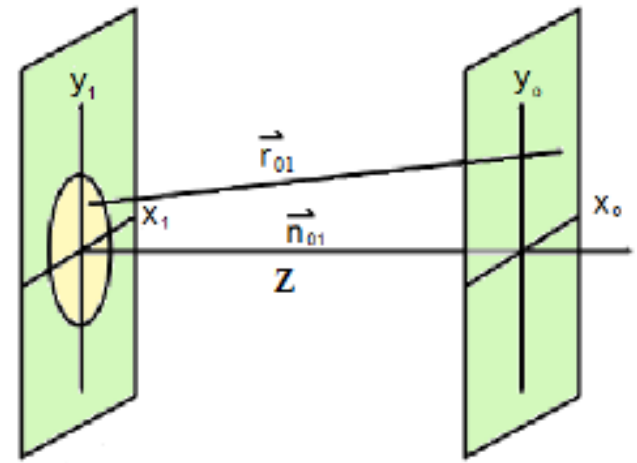

Figure 3.6: Basic Diffraction Geometry (after [41]) 
The Fresnel number, $F$, governs the appropriate diffraction model to adopt and is given by

$$
F=\frac{a^{2}}{\lambda \cdot \mid \overrightarrow{n_{01} \mid}}
$$

where $\lambda$ is the wavelength, $a$ is the radius of the aperture, and $\left|\overrightarrow{n_{01}}\right|$ is the distance of the observation plane from the aperture as seen in Figure 3.6.

If $F \approx 1$, Fresnel Diffraction is said to occur, and if $F \ll 1$, then Fraunhofer diffraction is assumed. Values of $F$ in between require a more detailed diffraction analysis.

If the field amplitude at $\left(x_{1}, y_{1}\right)$ in Figure 3.6 is given by $U\left(x_{1}, y_{1}\right)$, then it follows from the Huygens-Fresnel principle that the field amplitude at point $\left(x_{o}, y_{o}\right)$ is given by

$$
U\left(x_{o}, y_{o}\right)=\int_{\epsilon} \int h\left(x_{o}, y_{o} ; x_{1}, y_{1}\right) \cdot U\left(x_{1}, y_{1}\right) d x_{1} d x_{2}
$$

where

$$
h\left(x_{0}, y_{0} ; x_{1}, y_{1}\right)=\frac{1}{j \lambda} \frac{e^{j k\left|r_{01}\right|}}{\left|\overrightarrow{r_{01}}\right|} \cos \left(\overrightarrow{n_{01}}, \overrightarrow{r_{01}}\right)
$$

for wave number $k=\frac{2 \pi}{\lambda}$, aperture area $\epsilon$, and vectors $\overrightarrow{n_{01}}$ and $\overrightarrow{r_{01}}$ given by Figure 3.6 $[33,40,42]$.

In the Fresnel diffraction region, the electric field diffraction pattern at point $\left(x_{o}, y_{o}\right)$ is given by

$$
U\left(x_{o}, y_{o}\right)=\frac{\exp (j k z)}{j \lambda z} \iint_{-\infty}^{\infty} U\left(x_{1}, y_{1}\right) \exp \left(j \pi \frac{\left(x_{0}-x_{1}\right)^{2}+\left(x_{0}-x_{1}\right)^{2}}{\lambda z}\right) d x_{1} d y_{1} .
$$

It can be shown that the diffracted field is essentially the convolution of the aperture distribution with a spherical wave [40].

In the Fraunhofer Diffraction region $(F \ll 1)$, the electric field diffraction pattern at point $\left(x_{o}, y_{o}\right)$ is given by

$$
\begin{aligned}
U\left(x_{o}, y_{o}\right)= & \frac{\exp [j k z]}{j \lambda z} \exp \left[j \frac{k}{2 z}\left(x_{0}^{2}+y_{0}^{2}\right)\right] \\
& \iint_{-\infty}^{\infty} \exp \left[-j 2 \pi\left(x_{1} \frac{x_{0}}{\lambda z}+y_{1} \frac{y_{0}}{\lambda z}\right)\right] \cdot U\left(x_{1}, y_{1}\right) \cdot d x_{1} d y_{1} .
\end{aligned}
$$

It should be noted that Equation 3.14 essentially describes a weighted version of the Fourier Transform (see Equation 3.15) of the aperture distribution, evaluated at frequencies $(u, v)=\left(\frac{x_{0}}{\lambda z}, \frac{y_{0}}{\lambda z}\right)$. The expressions in Equations 3.13 and 3.14 follow from the HuygensFresnel principle with simplifications resulting from the diffraction models made. For more information, see [33, 40, 42]. 


\subsubsection{Fourier Transforming Property of a Thin Lens}

The two-dimensional Fourier Transform of function $g(x, y)$ is described by

$$
G(u, v)=\iint_{-\infty}^{\infty} g(x, y) e^{-j 2 \pi(u x+v y)} d x d y
$$

It will be seen that a thin lens embodies a Fourier transforming property.

A lens is made of optically dense material through which the velocity of the wave is less than in air. A thin lens is such that a wave entering the lens at coordinates $(x, y)$ emerges from the lens at roughly the same coordinates on the other side.

A thin lens delays the incident wave according to lens thickness at the point of entry, causing a phase delay that transforms the field distribution in front of the lens into the field distribution behind the lens.

The focal length, $f$, of an optical system is a measure of how strongly it converges or diverges light. A shorter focal length corresponds to greater optical power than a longer focal length. The focal length of a thin lens is defined by

$$
\frac{1}{f}=(n-1)\left[\frac{1}{R_{\text {in }}}-\frac{1}{R_{\text {out }}}\right],
$$

where $R_{\text {in }}$ and $R_{\text {out }}$ are the two radii of convergence of the lens (see Figure 3.7) and $n$ is the index of refraction. The focal length is positive for all converging lenses, such as those used in a camera, and negative for divergent lenses.

Photography is based on the principle that a sharp image is produced at a known distance behind the thin lens, as shown in Figure 3.7. The relevant distances are related as

$$
\frac{1}{S_{1}}+\frac{1}{S_{2}}=\frac{1}{f}
$$

This process underlines why cameras must be in focus to produce a sharp image (the observation plane in the camera should be positioned at the correct relative distance such that a sharp image is observed).

Before considering the PSF of a thin lens, it is worth exploring what occurs as a light wave passes through a thin lens. The phase delay imposed on the wave by the lens is what determines the intensity distribution at the observation plane.

Let $\Delta_{o}$ be the maximum thickness of the lens and $\Delta(x, y)$ be the thickness of the lens where the light enters (see Figure 3.8), such that

$$
\Delta(x, y)=\Delta_{o}-\frac{x^{2}+y^{2}}{2}\left(\frac{1}{R_{1}}-\frac{1}{R_{2}}\right) .
$$




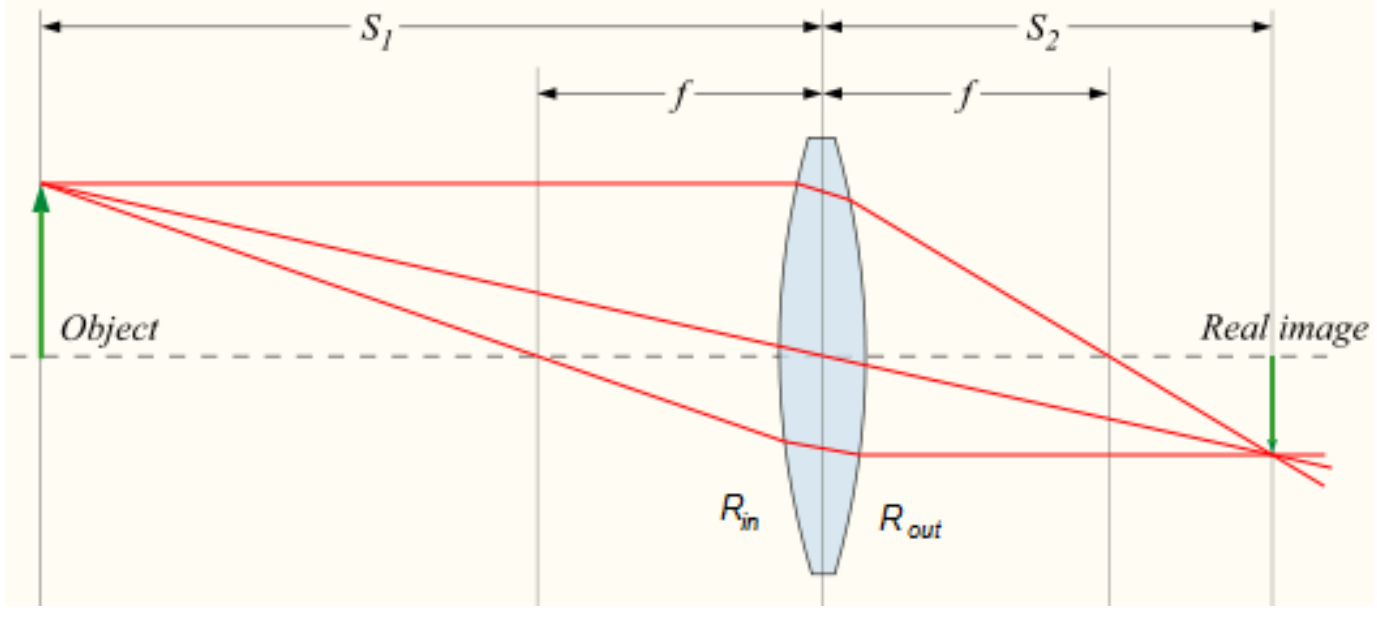

Figure 3.7: Thin Lens Parameters with Real Image (as in Photography) [43]

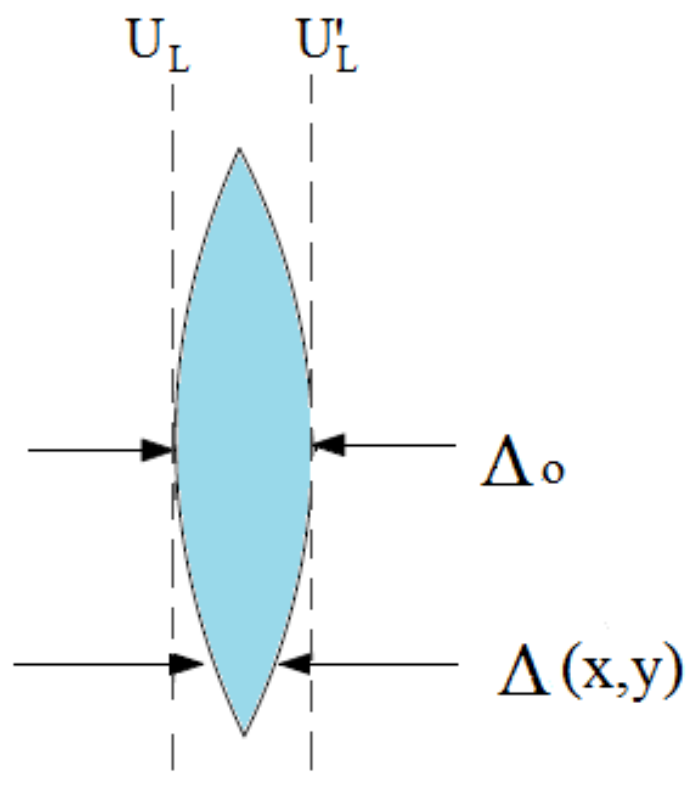

Figure 3.8: Lens Geometry (after [33]) 
The overall phase transformation of the lens is given by

$$
\begin{aligned}
t_{l}(x, y) & =e^{j k \Delta_{o}} e^{j k(n-1) \Delta(x, y)} \\
& =e^{j k n \Delta_{o}} e^{-j \frac{k}{2 f}\left(x^{2}+y^{2}\right)},
\end{aligned}
$$

which relates the field distribution behind the lens, $U_{l}^{\prime}$, to the field distribution in front of the lens, $U_{l}$, by

$$
U_{l}^{\prime}=t_{l}(x, y) \cdot U_{l}
$$

$[33,40]$.

The Fourier Transforming property of a lens is demonstrated by considering an object illuminated by monochromatic light, at a distance $S_{1}$ from a thin lens (as in Figure 3.7), but letting the observation plane be a distance $f$ (the focal length) behind the lens.

Equations 3.14 and 3.20 can then be combined to calculate the field amplitude at the observation plane, $U_{f}$. This relationship is given by

$$
U_{f}\left(x_{f}, y_{f}\right)=\frac{e^{j \frac{k}{2 f}\left(x_{f}^{2}+y_{f}^{2}\right)}}{j \lambda f} \cdot F_{l}\left(\frac{x_{f}}{\lambda f}, \frac{y_{f}}{\lambda f}\right),
$$

where $\left(x_{f}, y_{f}\right)$ are coordinates on the observation plane and $F_{l}$ denotes the Fourier transform of the light incident to the lens evaluated at the given frequencies. Some constant phase terms have again been dropped. The spectrum, $F_{l}$, is proportional to the Fourier Transform of the light transmitted by the object, $F_{o}$. This relationship results from Equation 3.11 and is given by

$$
F_{l}(u, v)=e^{-j k \lambda S_{1}\left(u^{2}+v^{2}\right)} F_{o}(u, v) .
$$

To summarize the entire process, the lens produces at its focal plane the Fraunhofer diffraction pattern of the original distribution. When the light passes through the lens, the field at the focal plane is the Fourier transform of the original distribution multiplied by a spherical wavefront and the transfer function of Equation 3.22.

It should now be clear to those familiar with radio communications that there are strong analogies between Fourier Optics and the Fourier analysis of electrical signals. The concept of a PSF and OTF are thus analogous to the impulse response and transfer function of an RF channel. 


\subsubsection{The PSF and OTF of a Thin Lens}

As discussed in Section 3.2.2, photography is based on the fact that, for a thin lens, a sharp image is produced on a plane at a known position. The distance from the lens to the image plane is related to the focal length of the lens and the distance from the object to the lens through Equation 3.17. The geometry for this scenario can be seen in Figure 3.7.

The PSF for the specific case of monochromatic illumination and a single thin lens in focus is represented as

$$
\begin{aligned}
h\left(x_{1}, y_{1}, x_{2}, y_{2}\right) & \cong \frac{1}{\lambda^{2} S_{1} S_{2}} . \\
& \iint_{\infty}^{\infty} I(x, y) \cdot \exp \left[\frac{-j k}{S_{2}}\left[\left(M x_{1}+x_{2}\right) x+\left(M y_{1}+y_{2}\right) y\right]\right] d x d y,
\end{aligned}
$$

where $I(x, y)$ is an indicator function that equals 1 if $(x, y)$ lies within the camera aperture and 0 otherwise. $M$ denotes the magnification of the system given by $M=S_{1} / S_{2}$. Realistically, the light will be spatially incoherent, meaning that the phasor amplitudes of the object vary independently with space.

For an imaging system with incoherent lighting and a circular aperture, the magnitudeof the optical transfer function (MTF) is described by

$$
H(\rho)=\frac{2}{\pi}\left[\cos ^{-1}\left(\frac{\rho}{2 \rho_{o}}\right)-\frac{\rho}{2 \rho_{o}} \sqrt{1-\left(\frac{\rho}{2 \rho_{o}}\right)^{2}}\right] \cdot U\left(2 \rho_{o}-\rho\right),
$$

where $\rho$ denotes the radial displacement equal to $\sqrt{f_{x}^{2}+f_{y}^{2}}, \rho_{o}$ denotes the cutoff frequency equal to $D /\left(2 \lambda S_{2}\right)$ ( $\mathrm{D}=$ diameter of the aperture), and $U(x)$ is a unit step function equal to 1 if $x \geq 0$ and 0 otherwise. The MTF is displayed in Figure 3.9. (See [44] for further detail.)

The low-pass characteristic of the channel can clearly be observed from Figure 3.9. However, the shape of the OTF magnitude will not realistically correspond to the figure. Further consideration must be made to account for the fixed-focus characteristic common for cell phone cameras, as well as relative motion between the camera and the barcode image while the shutter is open.

\subsubsection{Defocused Images}

Recall that the image distance for a thin lens is governed by Equation 3.17 and illustrated by Figure 3.7. Now consider the practical scenario in photography in which the camera sensor 


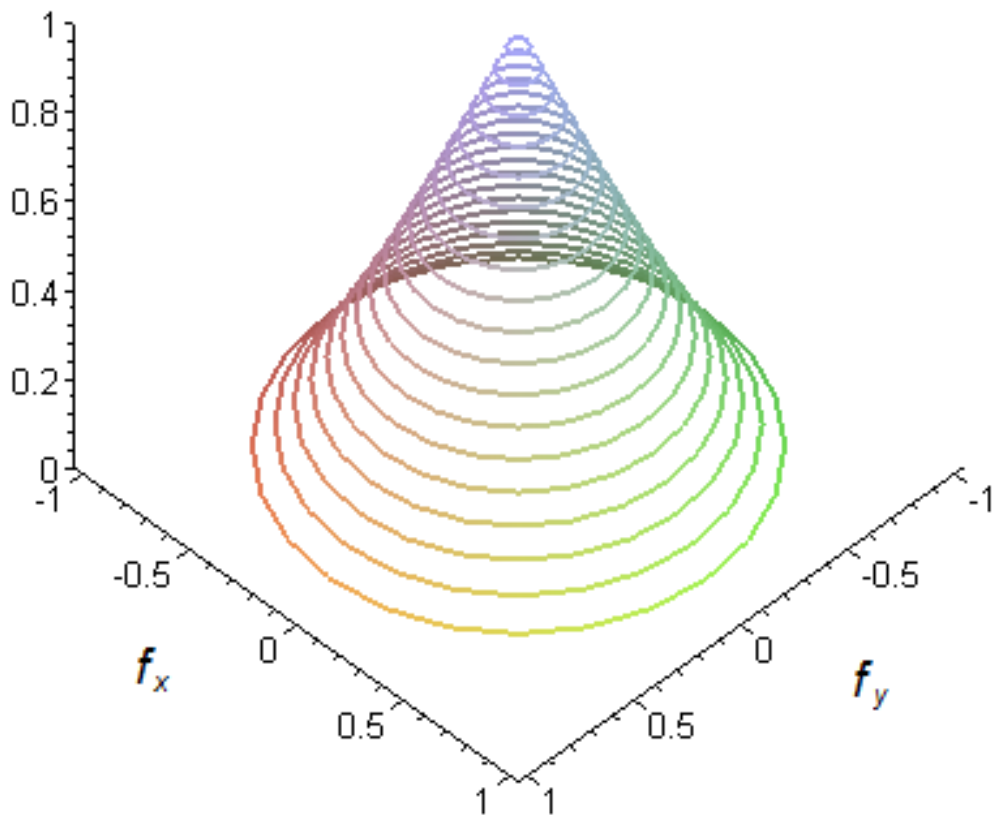

Figure 3.9: Lens MTF

MTF for Lens with Circular Aperture and Incoherent Lighting 


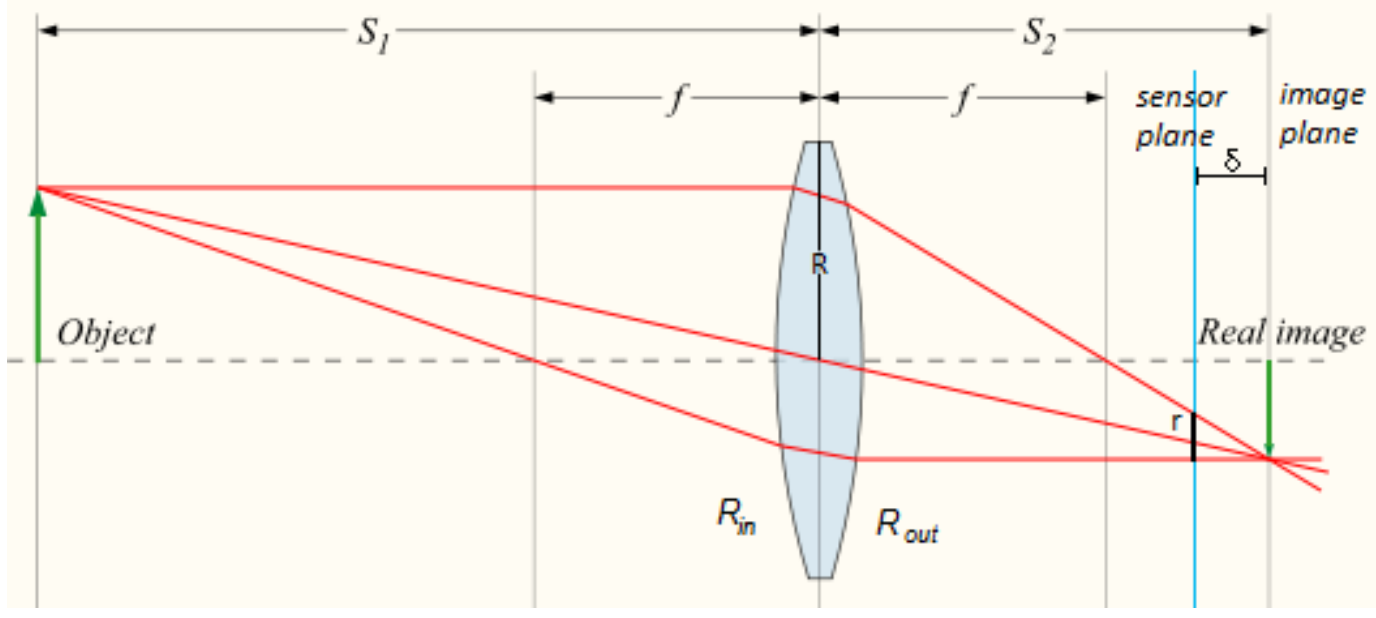

Figure 3.10: Formation of a Defocused Image versus Focused Image (after [43])

plane does not coincide with the image plane. This set-up is illustrated in Figure 3.10, in which the sensor plane is displaced from the image plane by distance $\delta$.

For a circular aperture, the energy is distributed on a circular patch of radius $r$ on the sensor plane (see Figure 3.10). The displacement of the sensor plane relates the radius of the patch to the radius of the lens by

$$
r=\frac{\delta R}{S_{2}}
$$

The distribution of light energy over the patch is often referred to as a blurring function, and is typically approximated by a two-dimensional Gaussian function $[45,46,47]$. Under this model, the defocused image, $I_{d}(x, y)$, can be described by convolving the focused image on the image plane, $I_{f}(x, y)$, with the Gaussian blurring function, as expressed by

$$
I_{d}(x, y)=h(x, y) * I_{f}(x, y)
$$

where

$$
h(x, y)=\frac{1}{2 \pi \sigma_{h}^{2}} e^{-\frac{x^{2}+y^{2}}{2 \sigma_{h}^{2}}} .
$$

The variance $\sigma_{h}^{2}$ of the Gaussian blur function is proportional to the radius of the patch, described by Equation 3.25.

The relationship between the focused and defocused images of Equation 3.26 can equally be described in the frequency domain $(u, v)$ by

$$
I_{D}(u, v)=H(u, v) \cdot I_{F}(u, v)
$$




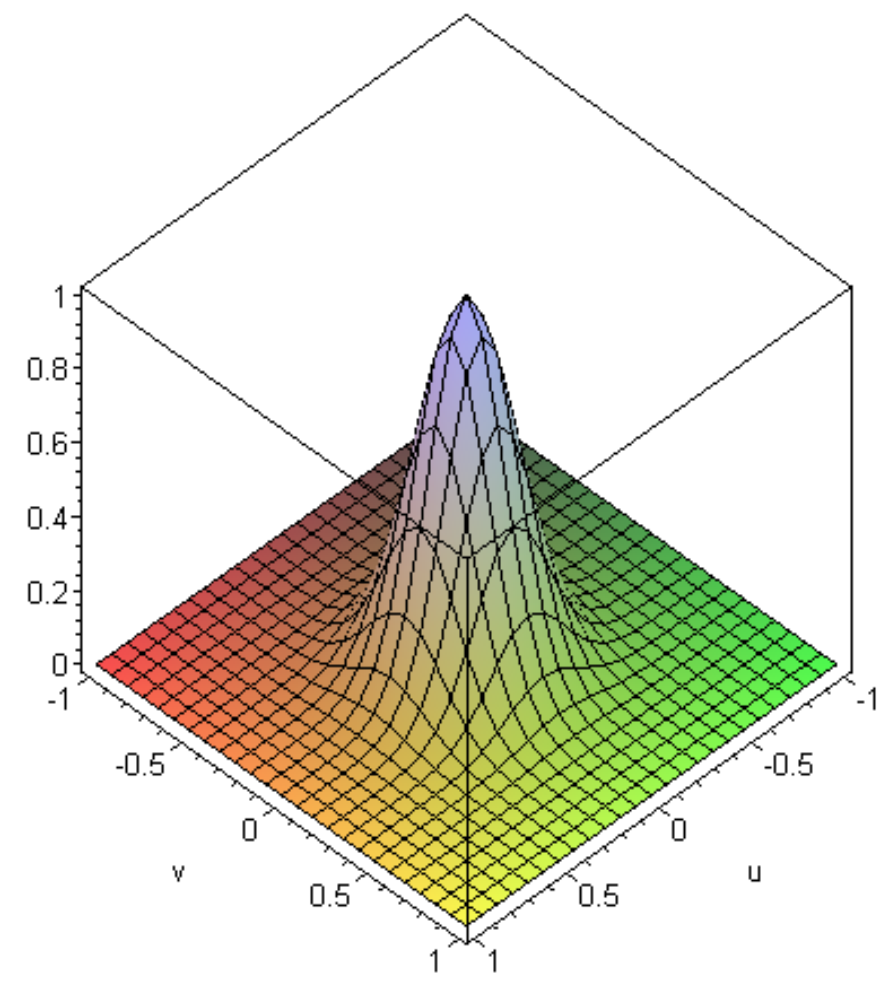

Figure 3.11: MTF due to Defocus

where

$$
H(u, v)=e^{-\frac{u^{2}+v^{2}}{2} \sigma_{h}^{2}}
$$

The transfer function of Equation 3.29 passes low frequencies and attenuates higher frequencies, creating, again, a two-dimensional low-pass filter as illustrated in Figure 3.11. As the image plane is displaced further, $\delta$ increases, thus increasing the radius of the patch and the variance of the transfer function. This causes the image to experience more blur.

In order to prevent defocus blur when a photograph is taken, the camera should be focused on the subject of interest. In the resulting photo, the object will appear in focus, as will objects of a certain distance around it. The depth of field refers to the range of distances for which objects are in focus. Although a lens can only focus precisely at one distance, the depth of field indicates how gradual the decrease in sharpness is on either side of the focus distance.

Camera lenses are typically defined by their $F$ number (f/\#), which is the ratio of the 
focal length to the diameter of the aperture. In a single-lens reflex camera, the aperture size (entrance pupil) is adjustable, allowing the photographer to change the $\mathrm{f} / \#$. Changing the $\mathrm{f} / \#$ controls the amount of light hitting the imager to create proper light conditions. Increasing or decreasing the $\mathrm{f} / \#$ also causes the depth of field to increase or decrease.

Camera phones have a fixed $\mathrm{f} / \#$, meaning that the aperture size (entrance pupil) cannot be adjusted, which limits the imaging conditions available to the photographer. Although cell phone cameras have small apertures, they also have small focal lengths due to their compact size. This results in relatively low f/\#s. For example, the Nokia 6600 cell phone has a camera with a $3.5 \mathrm{~mm}$ focal length and 1.25 aperture diameter, giving an $\mathrm{f} / \#$ equal to 2.8 (often written as $\mathrm{f} / 2.8$ ) which is typical for mobile phone cameras. This corresponds to a small depth of field, so few objects will be sharp in the image.

A small $\mathrm{f} / \#$ coupled with fixed-focus limits the potential for a sharp picture of the barcode. Without the ability to focus, the sharpness of the barcode photo will strictly depend on where the photographer is standing. For this reason, it is crucial that the blur function be considered in the channel model and that the barcode is not assumed to be in focus.

\subsubsection{Motion Blur}

Still photography assumes that there is no relative motion between the object being photographed and the camera while the camera shutter is open. However, this is often not the case in practice, particularly for photographs taken with a cell phone. Cell phone photos are generally snapped quickly with one hand holding the phone. The result is jitter causing the object to appear across several of the imaging pixels.

Recall that a degradation process can be described in the frequency domain by the relationship

$$
D(u, v)=H(u, v) \cdot I(u, v),
$$

where $D(u, v)$ is the Fourier transform of the degraded image, $H(u, v)$ is the degradation transfer function, and $I(u, v)$ is the unblurred image.

Consider the degradation transfer function due to motion. For a camera with mechanical shutter and relative motion along the column axis, this function can be described by

$$
H(u, v)=\frac{\sin \left(\pi S T \sqrt{u^{2}+v^{2}}\right)}{\pi S T \sqrt{u^{2}+v^{2}}},
$$




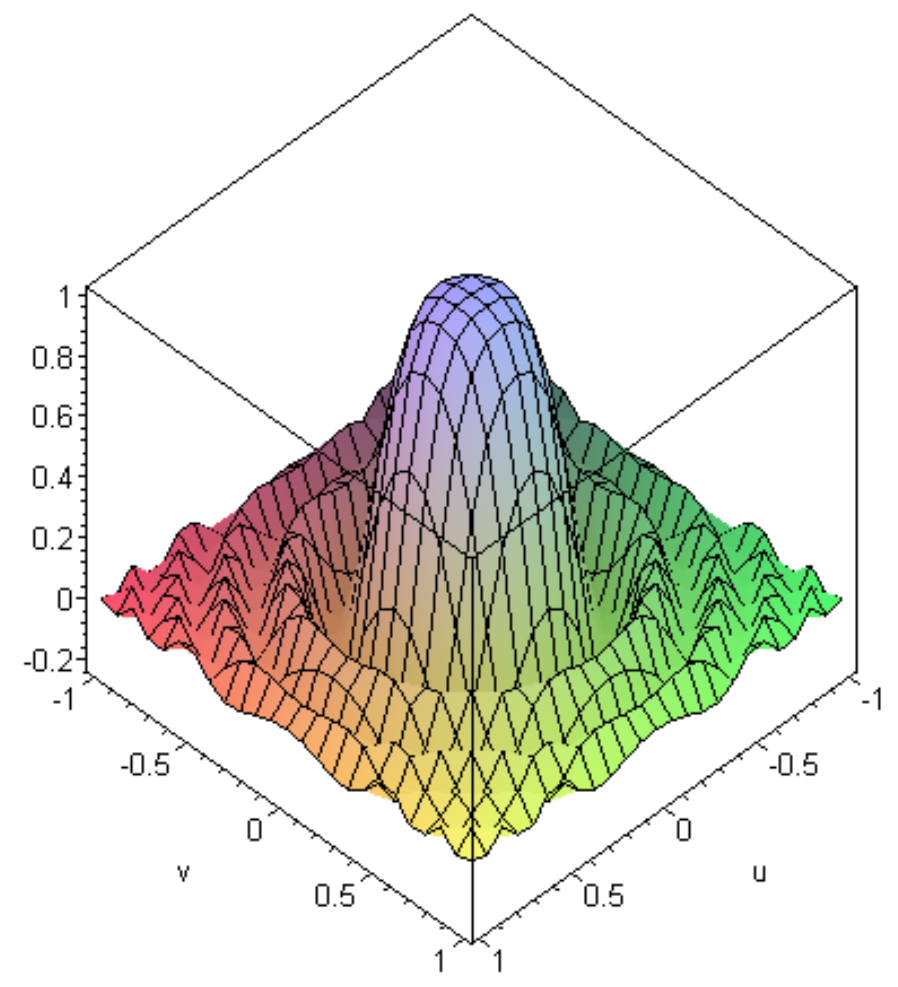

Figure 3.12: MTF Due to Motion Blur

where $S$ is the constant speed in direction of the column axis and $T$ is the time interval for which the shutter is open. This motion-blur MTF is illustrated Figure $3.12[48,49,50]$.

Note that the transfer function is a sinc function $(\sin x / x)$, which is the Fourier Transform of a rectangular function (the constant speed in one direction results in a uniform PSF). The transfer function can be modified to account for motion in other directions. It can also be adjusted to account for the finite amount of time it takes for the aperture to open and close (opening/closing is not instantaneous as suggested by the rectangular function). However, Equation 3.31 is a sufficient model for motion blur, particularly because the direction of motion is of no direct influence over the resulting image quality of the barcode.

\subsubsection{Overall Optical Transfer Function}

The overall optical transfer function can now be defined as the product of the transfer functions of Equations 3.24, 3.29, and 3.31. Recall, the aberration-free ideal system gives transfer 


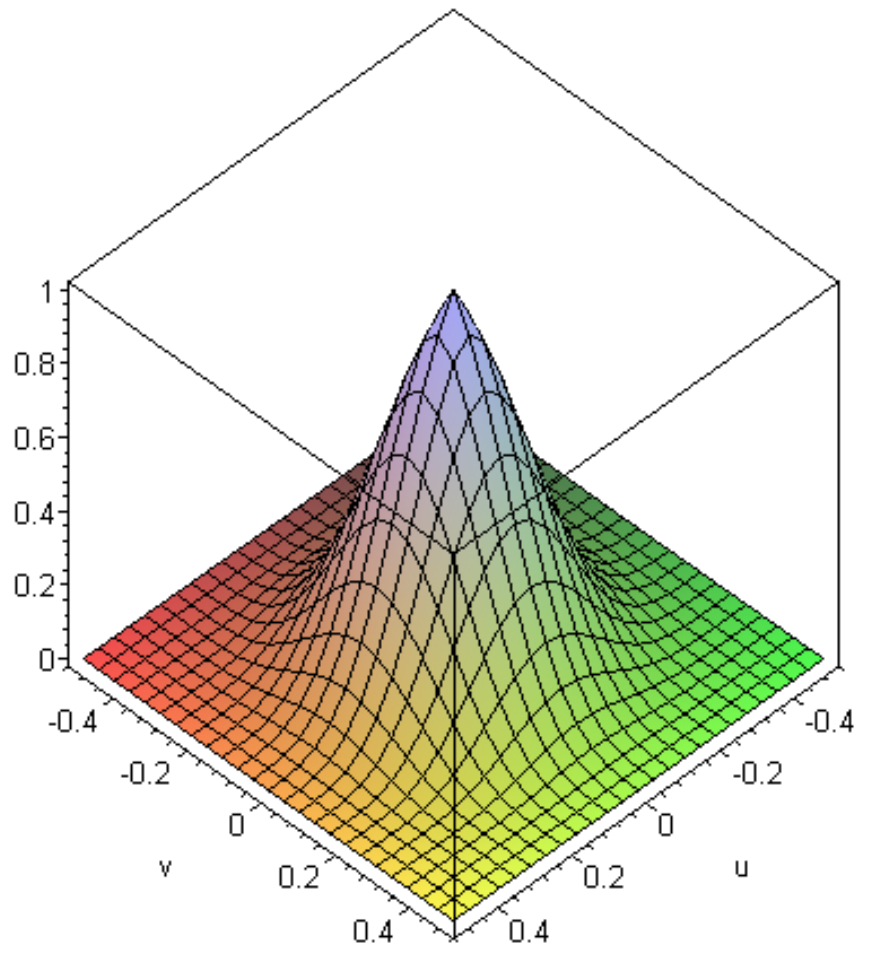

Figure 3.13: Overall Transfer Function of Camera Lens 
function

$$
\begin{aligned}
H_{\text {ideal }}(u, v)=\frac{2}{\pi}\left[\cos ^{-1}\left(\frac{\sqrt{u^{2}+v^{2}}}{\rho_{o}}\right)-\right. & \\
& \left.\frac{\sqrt{u^{2}+v^{2}}}{\rho_{o}} \sqrt{1-\left(\frac{\sqrt{u^{2}+v^{2}}}{\rho_{o}}\right)^{2}}\right] \cdot U\left(\rho_{o}-\rho\right),
\end{aligned}
$$

where $U(x)$ is the unit step function equal to 1 if $x \geq 0$ and 0 otherwise.

The cut-off frequency for a lens, denoted by $\rho_{o}$ in Equation 3.32, is equal to the inverse of the product of the wavelength of light and the $\mathrm{f} / \#$ of the lens:

$$
\rho_{o}=\frac{1}{\lambda \cdot f / \#}
$$

(Note that Equation 3.32 is equivalent to Equation 3.24 with $\rho_{0}$ scaled by a factor of 2 and redefined for $S_{1} \rightarrow \infty$.)

For a typical cell phone f/\# of 2.8 and the visible spectrum of $400-700 \mathrm{~nm}$, the result is a maximum cut-off frequency of 0.510 cycles/micrometer for an ideal, aberration-free lens. For colour images, the cut-off frequency will be different for each colour due to wavelength differences.

It was shown that, due to the fixed-focus and limited depth-of-field characteristics of cell phone cameras, defocus blur is to be expected. Equation 3.29 expressed the Gaussian transfer function typically used to model defocus blur in images. Recall that $\sigma_{h}$, the standard deviation in Equation 3.27, is proportional to the radius of the patch on the displaced sensor plane. Consider the Nokia 6600 cell phone with $3.5 \mathrm{~mm}$ focal length and $1.25 \mathrm{~mm}$ aperture. A $0.028 \mathrm{~mm}$ displacement between image plane and image sensor results in a $5 \mu \mathrm{m}$ patch radius. A reasonable standard deviation for the Gaussian blur model could be $5 \mu \mathrm{m}$, as demonstrated in defocus analysis by Claxton and Staunton (see [47]). This gives the defocus transfer function,

$$
\begin{gathered}
H_{\text {defocus }}(u, v)=e^{-\frac{u^{2}+v^{2}}{2} \sigma_{h}^{2}} \\
\sigma_{h}=5 .
\end{gathered}
$$

Finally, it was seen that relative motion between the object and camera while the shutter is open will cause a further blurring effect. Assuming the total displacement is $5 \mu \mathrm{m}$ 
along the $(x, y)$ axis for a shutter speed of 1/60 seconds (a typical maximum for cell phone cameras) and hand velocity of $0.3 \mathrm{~mm} / \mathrm{s}$, Equation 3.31 becomes

$$
H_{\text {motion }}(u, v)=\frac{\sin \left(\pi 5 \sqrt{u^{2}+v^{2}}\right)}{\pi 5 \sqrt{u^{2}+v^{2}}} .
$$

The resulting OTF is denoted by

$$
H_{\text {overall }}(u, v)=H_{\text {ideal }} \cdot H_{\text {defocus }} \cdot H_{\text {motion }}
$$

and illustrated in Figure 3.13.

\subsection{CMOS Camera Response}

Charge-Coupled Devices (CCDs) have traditionally dominated image-sensor technology and were originally the sensor of choice for cell phones. However, recent advances in the design of CMOS image sensor technology led to their adoption in several high-volume products, including mobile phones. Before discussing the non-idealities that limit CMOS imager sensors and contribute to distortion in the channel model of Equation 3.1, an introduction into sensor operation is required.

\subsubsection{CMOS Imagers}

A CMOS image sensor, seen in Figure 3.14, is a pixelated metal oxide semiconductor that converts an optical image into an electrical signal.

To perform colour imaging, a color-filter-array (CFA) is placed in front of the sensor. Although there are other mechanisms of imaging colour, the Bayer colour filter is the solution of choice for low-cost cameras, such as those in mobile phones.

In the CMOS imaging system seen in seen in Figure 3.15, the Bayer array is responsible for arranging a colour filter (of type red (R), green $(\mathrm{G})$, or blue (B)) over the sensor pixels. The Bayer filter is a periodic array built from a 2-by-2 pixel kernel in a RGBG pattern. Therefore, half of the pixels are green, leaving red and blue pixels to each represent one quarter of the total number. Because each pixel is only made sensitive to one colour of the three primary colours in the RGB scheme, colour image sensors have one third the sensitivity of monochrome sensors. 


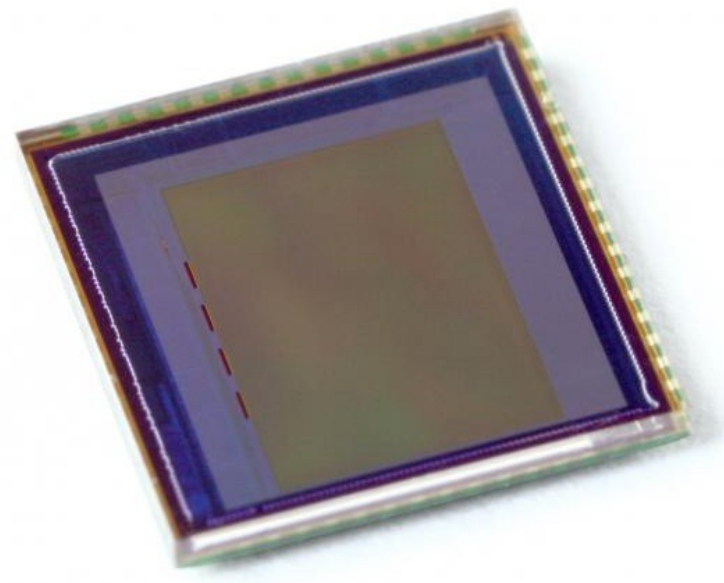

Figure 3.14: A CMOS Image Sensor [51]

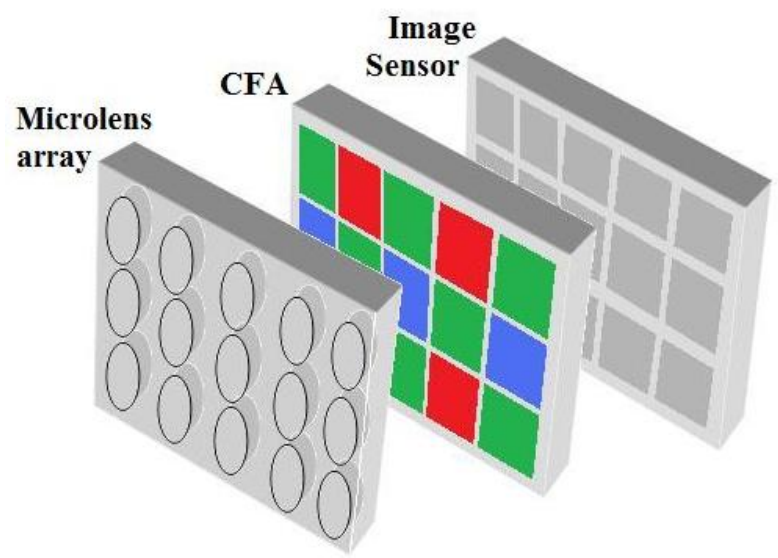

Figure 3.15: CMOS Sensor Array Preceded by Microlens Array and Colour Filter Array (CFA) 
Just as the lens of the camera focuses light onto the image plane, an array of microlenses serves to concentrate the photons onto the photosensitive portion of the pixels. The CFA is placed in between the array of microlenses and the sensor (see Figure 3.15).

To convert the image from Bayer format (where each pixel represents one colour only) to the standard RGB image, interpolation is required to assign three colour values, $(R, G, B)$, to each pixel. The CMOS image cameras employ a process termed demosaicing to determine missing colour values from neighbouring pixels. Although many demosaicing methods are possible, bilinear interpolation, where the neighbouring pixels are weighted and averaged to assign the new pixel value, is widely used due to its simplicity and relative accuracy [52]. Unfortunately, because the bilinear interpolation demosaicing process does not consider the presence of object boundaries and indiscriminately combines colour values across edges, errors are inevitable.

The CMOS image sensor consists of an array of pixels, each containing a photodetector that converts incident light into photocurrent. The photodetectors in the pixels are typically reverse-biased positive-negative $(\mathrm{PN})$ junction photodiodes or $\mathrm{P}+/ \mathrm{N} / \mathrm{P}$ pinned diodes. The process carried out by the photodetectors is described by the photoelectric effect. When light strikes a semiconductor, photon energy is absorbed and the resulting electric field causes electrons to be freed, creating a current (see Figure 3.16) [53]. In addition to a photodetector, each pixel also contains the readout circuits needed to convert the photocurrent into a voltage to be read off the array.

To summarize, the CMOS sensor accumulates a signal charge in each pixel proportional to the local illumination intensity, performing a spatial sampling function. A charge-tovoltage conversion is done in each pixel. The result is a digital representation of the incident light on each pixel.

In a CMOS imager, the charge voltage signals are read out one row at a time using row and column circuits. The readout path entails several active devices that introduce both temporal and fixed-pattern (FPN) noise. The advantage to the random access readout is that it allows for high-speed readout and window-of-interest operations at low power consumption. However, the noise introduced has a significant impact on the pixel signal fidelity and therefore on the overall quality of photographs taken. The noise sources of the image sensor must be taken into careful consideration when modelling the channel. 


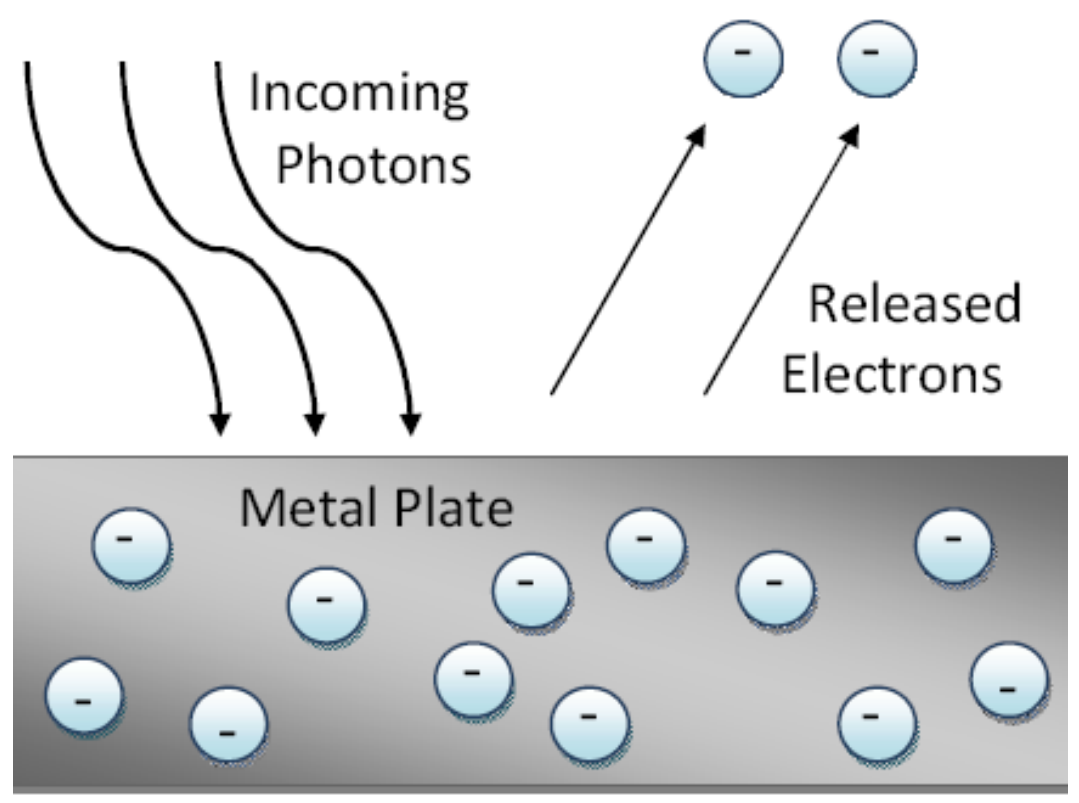

Figure 3.16: Illustration of the Photoelectric Effect (after[33])

\subsubsection{Nyquist Sampling Theory and Cut-Off Frequency}

The Nyquist Sampling Theorem is well known in signal processing. It dictates the sampling rate necessary to preserve the information contained in the signal. For a signal containing higher frequency components, it is intuitive that it would be necessary to sample at a higher rate. The Nyquist Theorem states that, in order to preserve the full information in the signal, it is necessary to sample at twice the maximum frequency of the signal. This cut-off frequency is commonly referred to as the Nyquist frequency.

The Nyquist sampling concept also applies to the spatial domain when sampling images, as done by the CMOS array in a camera. The spacing between pixels in a CMOS array, meaning the distance from the center of a pixel to the center of its neighbouring pixel, is defined as pixel pitch. Following the Nyquist Sampling theorem, for a given CMOS array with pixel pitch $p$, the cut-off frequency can be described by

$$
F_{\text {cutoff }}=\frac{1}{2 p} \text {. }
$$

Spatial frequencies above the cut-off frequency will cause aliasing. The spatial domain representation of the Nyquist Sampling theorem in Equation 3.37 is intuitive: a higher cutoff frequency corresponds to more closely spaced pixels (samples). 
Recent advances in CMOS technology have made smaller pixel design possible, which can improve the spatial resolution of an imager by increasing the number of pixels in a fixed sensor area. The trade-off is that smaller pixels make the imager more susceptible to crosstalk. Additionally, the signal-to-noise ratio (SNR) of the pixels is reduced because their smaller size means they receive a lesser photon flux.

The object-pixel defines the smallest recordable element in the image space, which is often related to detector size. The object-pixel size strongly influences the magnitude of the OTF. Consider the case where an object pixel represents a point image. It follows that the pixel shape determines the point spread function. The Transfer function of such a system would be a normalized Fourier transform of the pixel shape. For a rectangular pixel array, the magnitude of the OTF (MTF) is the well-known sinc function, described by

$$
H(w)=\frac{a}{p} \frac{\sin \left(\frac{w a}{2}\right)}{\left(\frac{w a}{2}\right)}
$$

for pixel pitch $p$, pixel size $a$, and angular displacement $w[54,55]$.

CMOS image sensors generally suffer from a low fill-factor (FF), which is the ratio of photo-sensing area ( $a^{2}$ for $a$ in Equation 3.38) in each pixel to the total area of the pixel ( $p^{2}$ for $p$ in Equation 3.38). Note that if the pixel size is equal to the pitch, Equation 3.38 reduces to $H(w)=\operatorname{sinc}\left(\frac{w p}{2}\right)$. Decreasing the pixel size increases the bandwidth of the system and broadens the MTF, meaning that, spatially, finer details can be resolved.

For colour image sensors employing a CFA, such as the CMOS sensors in mobile phones, the cut-off frequency in each of the three colour channels is significantly reduced. Because the interpolation process implicitly assumes that the sensors in the kernel measure a common spectral radiance in the scene, the effective sampling rate is lower. The cut-off frequency is lower for the red and blue channels than it is for the green due to twice as many green pixels in the sensors. It follows that, in order to avoid aliasing, the under-sampling imposed on each of the three channels by the Bayer filter must be considered. Overall, the spatial resolution is decreased.

\subsubsection{Sampling}

When sampling a continuous image, as done by the CMOS sensor to produce a digital image, the image is sampled in both vertical and horizontal coordinates and the amplitude 
at each sampled coordinate $\left(x_{i}, y_{i}\right)$ is quantized. The result of the sampling and quantization process is a matrix of real numbers.

Assume that an image $f(x, y)$ is sampled such that the resulting digital image has $M$ rows and $N$ columns. The discrete values of the coordinates $(i, j)$ in digital image matrix $A$,

$$
A=\left[\begin{array}{cccc}
a_{0,0} & a_{0,1} & \ldots & a_{0, N-1} \\
a_{1,0} & a_{1,1} & \ldots & a_{1, N-1} \\
\vdots & \vdots & & \vdots \\
a_{M-1,0} & a_{M-1,1} & \ldots & a_{M-1, N-1}
\end{array}\right],
$$

indicate the quantized intensity values, as denoted by $a_{i, j}$ where $a_{i, j}=f(x=i, y=j)$.

Sampling is the principal factor in determining the spatial resolution of the image, while gray-level resolution refers to the smallest discernible change in a gray level (note that this can be a subjective measuring process). The level of quantization applied to the intensity values will determine the gray-level resolution and is generally a power of two. For an $M \times N$ digital image with $2^{k}$ gray-levels, $b=M \times N \times k$ bits are required to represent the image.

Sampling generally refers to a mapping from a continuous image representation to a digital one. However, sampling can also be applied to a digital image to increase or decrease the number of pixels used to represent the image. The two processes can be referred to as up-sampling and down-sampling, respectively.

For the case of a barcode being photographed, the sampling applied by an $M_{2} \times N_{2}$ pixel imaging system will map the $M_{1} \times N_{1}$ barcode image to its approximated $M_{2} \times N_{2}$ representation. Generally, the cell phone camera will reduce the spatial resolution of the barcode $\left(M_{2}<M_{1}\right.$ and $\left.N_{2}<N_{1}\right)$. However, if the barcode is displayed on a liquid crystal display (LCD) or a cathode-ray tube (CRT) computer screen, such as on a website, the spatial resolution of the camera will be higher.

The up-sampling and down-sampling process will depend on how many camera pixels actually capture the barcode as opposed to background scenery. Optimally, all $M_{2} \times N_{2}$ pixels are used to represent the barcode, and no background scene is captured by the photograph. Clearly, this is unrealistic and should not be expected.

Down-sampling will undoubtedly occur for photographs of printed barcodes because the resolution of printers is much higher than that of typical camera phones. Down-sampling 
is similar conceptually to sampling a continuous image but can be performed using interpolation techniques.

One common method of down-sampling a digital image is row-column deletion. The method is simple: evenly-spaced rows/columns are deleted from the $2 \times 2$ image matrix until the desired number of pixels is achieved. Row-column deletion works best for downsampling by an integer value, and although fast, may result in a noticeably lower quality image.

To down-sample by a non-integer value in a more precise manner, bilinear interpolation can be applied to the original image. This is accomplished by taking an $M_{2} \times N_{2}$ grid and expanding it to a $M_{1} \times N_{1}$ grid that fits over the original image. Gray-level bilinear interpolation is done to determine the $M_{2} \cdot N_{2}$ pixel values, and then the grid is returned to its original $M_{2} \times N_{2}$ size, now containing the down-sampled barcode.

Bilinear interpolation only considers the four nearest pixels to the location of interest when calculating the gray value. Neighbours are weighted according to their distance to the location of interest. Much more sophisticated and accurate approaches exist, such as bicubic interpolation and other spline interpolation methods, which consider larger neighbourhoods of pixels, as well as rates of change in the gray levels surrounding the pixel of interest.

Up-sampling will presumably be the sampling process applied to the barcode for CRT or LCD transmitters. Pixel replication is a method of increasing the spatial resolution of an image through nearest-neighbour interpolation, and is the up-sampling analog to rowcolumn deletion. It is applicable when the image is increased by an integer number of times, and is done by duplicating each column (or row). Although this is again a fast method, it produces an objectionable checkerboard effect and is not representative of the channel.

The up-sampling done by the CMOS camera to a barcode displayed on an LCD is more appropriately modelled by a bicubic or bilinear interpolation method. Bicubic interpolation considers the four nearest pixels to the location of interest, eight derivatives (each corresponding to a vertical or horizontal direction from the four nearest pixels), and four cross-derivatives, when calculating the new grayscale value. For more information, see $[50,56,57]$.

For clarity of the sampling concepts, colour sampling was not included in the discus- 


\begin{tabular}{ccc}
\hline \hline Sensor & Surface & Gamma \\
\hline CCD chip & Silicon & 1.0 \\
Vidicon Tube & $\mathrm{Sb}_{2} \mathrm{~S}_{3}$ & 0.6 \\
Film & Silver halide & $>1.0$ or \\
& & $<1.0$ \\
\hline \hline
\end{tabular}

Table 3.1: Typical Gamma Values [38]

sion. The same principles apply for the Bayer filter camera. However, due to the RGB pattern, each colour channel requires its own sampling (up or down) mechanism. This colour sampling is discussed further in Section 3.4.2. For more information on sampling, see $[50,56,57]$.

\subsubsection{Nonidealities and Dynamic Range}

Spatial resolution, discussed in Section 3.3.2, relates to how sharply objects appear in the image and the degree of detail distinguished from the image. However, it does not indicate the range of light levels recordable by the camera. Dynamic Range refers to the difference between the brightest and dimmest light levels a camera can record in a single photograph.

Dynamic range is a critical figure of merit for image sensors. The dynamic range of an image sensor is often not wide enough to capture scenes with both high lights and dark shadows. This is especially true for CMOS sensors, which, in general, have lower dynamic range than CCDs.

It is generally desirable that the relationship between the input photons and the output voltage be linear. The relationship between input image and output image is often described by

$$
\text { output }=\text { gain } \cdot(\text { input })^{\gamma}+\text { offset, }
$$

where $\gamma$ is called the gamma of the recording medium. A truly linear recording system must have $\gamma=1$ and offset $=0$. Although the offset is almost never zero, most silicon-surfaced sensors do have a unity $\gamma$. Table 3.1 compares the gamma values for some well-known sensor types. 


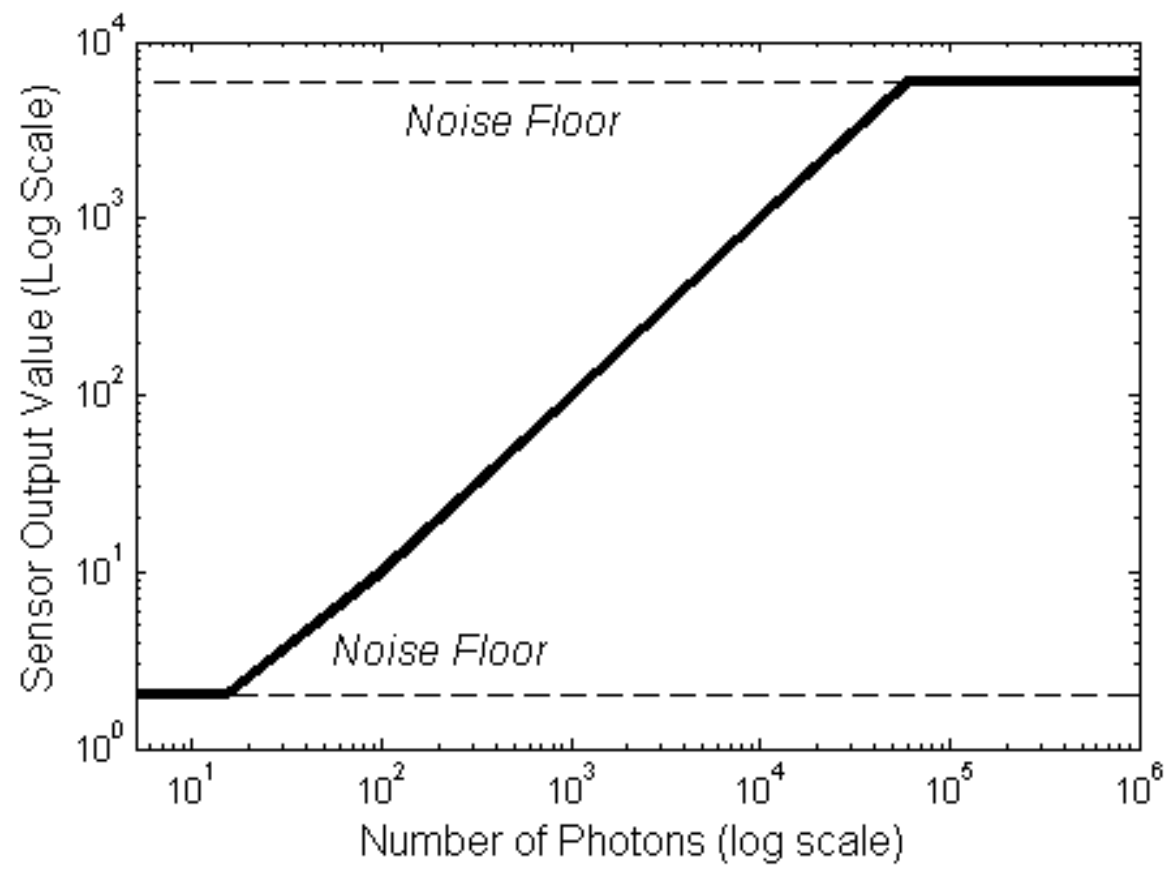

Figure 3.17: Sensor Dynamic Range and Region of Linear Responsivity (after [59])

Although there are logarithmic photodiode CMOS sensors, reversed-bias PN photodiodes and pinned photodiodes have a linear charge-to-voltage relationship [54,58]. Logarithmic photodiodes increase the dynamic range of the sensor, but are more expensive and have an adverse affect on pixel SNR. Therefore, they are generally used for advanced imaging systems that specifically require a high dynamic range (i.e. medical imaging machinery).

Dynamic range, the ratio of a pixel's saturation level to its signal threshold, is limited by the noise floor and the well capacity of the photodiode. Figure 3.17 demonstrates the dynamic range concept and indicates the noise floor and well capacity. Temporal noise and FPN are what determine the sensor's dynamic range and ultimately dictate the pixel signal fidelity.

Temporal noise, the primary non-ideality of an image sensor, sets a fundamental limit on image sensor performance, especially under low illumination. Temporal noise is independent across pixels and varies from frame to frame. For CMOS sensors, temporal noise arises from photodetector shot noise, pixel-reset circuit noise, readout circuit thermal and flicker noise, and quantization noise. Because temporal noise is random and not fixed, it 
cannot be overcome easily.

FPN is the variation in pixel outputs under uniform illumination. This is a result of interconnect mismatches across the sensor array. FPN can be broken down into offset FPN (independent of pixel signal) and gain FPN (increases with signal level).

Offset FPN is fixed for a given sensor array. However, the offset FPN varies from one array to the next. To overcome the effects of offset FPN, correlated double sampling (CDS) is typically done. CDS removes systematic offsets by comparing samples taken from the same pixels before and after integration (therefore, two samples are taken from each pixel instead of one).

Saturation is induced by the full-well capacity of the photodiode. The photodiodes in CMOS pixels operate in charge-integrating mode and therefore have a limited charge handling capacity. The full-well capacity, measured in electrons, is the maximum amount of charge that can be accumulated by a photodiode capacitance and is described by

$$
N_{\text {sat }}=\frac{1}{q} \int_{V_{\text {reset }}}^{V_{\max }} C_{P D}(V) \cdot d V
$$

where $C_{P D}$ is the photodiode capacitance, $q$ is the charge of an electron, and $V_{\text {reset }}$ and $V_{\max }$ are the initial and maximum voltages, which depend on photodiode structures and the operating conditions [60].

The root mean square (RMS) noise floor is also expressed in electrons. The dynamic range is the ratio of full-well capacity to noise floor (generally expressed in decibels). Typical CMOS image sensors have dynamic ranges of 40 to $100 \mathrm{~dB}[61,62]$.

Figure 3.18 lists the sources of noise for the sensor under dark and illuminated conditions. Note that in the saturation region, smear and blooming become a factor. Blooming occurs when the photodiode well is filled and additional photoelectrons spill over into adjacent pixels, causing the image to smear. The greater the region between pixels on the sensor, the more anti-blooming is achieved. The trade-off, of course, is a reduced spatial resolution.

Dark current, observed when the object is not illuminated, is an undesirable current that is integrated as dark charge at a charge storage node inside the pixel. The amount of dark charge has a dependency on temperature and is proportional to the integration time. Dark current is represented mathematically by

$$
N_{\text {dark }}=\frac{Q_{\text {dark }}}{q}=\frac{I_{\text {dark }} \cdot t_{\text {integration }}}{q}
$$




\begin{tabular}{|c|c|c|c|}
\hline & \multirow[t]{2}{*}{ Dark } & \multicolumn{2}{|c|}{ Illuminated } \\
\hline & & Below Saturation & Above Saturation \\
\hline \multirow{3}{*}{ 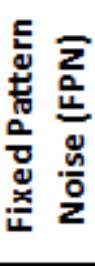 } & \multicolumn{2}{|c|}{$\begin{array}{l}\text { Dark Signal Nonuniformity } \\
\text { Pixel-Random Shading }\end{array}$} & \\
\hline & \multicolumn{2}{|c|}{$\begin{array}{c}\text { Dark Current Nonuniformity } \\
\text { (Pixel-wise, Row-wise, and Column-wise FPN) }\end{array}$} & \\
\hline & \multicolumn{3}{|c|}{ Defects } \\
\hline \multirow{4}{*}{ 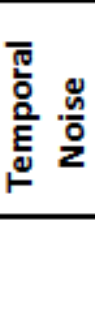 } & Dark Current Shot Noise & Photon Shot Noise & \\
\hline & \multicolumn{2}{|c|}{$\begin{array}{c}\text { Read Noise (Noise Floor) } \\
\text { Amplifier Noise, etc. } \\
\text { (Reset Noise) }\end{array}$} & \\
\hline & & & Smear, Blooming \\
\hline & \multicolumn{3}{|c|}{ Image Lag } \\
\hline
\end{tabular}

Figure 3.18: CMOS Image Sensor Sources of Noise Under Dark and Illuminated Conditions [60]

The dark current changes the output level corresponding to no illumination. This is evident in Figure 3.17 where a non-zero output voltage arises for no photon input.

It is crucial to carefully consider the dynamic range of the CMOS sensor into the overall channel model. The noise floor for a CMOS sensor is higher than that of a CCD sensor, reducing its performance in low light conditions. Cell phones are generally not equipped with a flash, adding to the problem. Overall, the cell phone camera can be anticipated to have poor performance in low light $[54,58,63,64]$.

\subsubsection{Modelling the Dynamic Range}

Figure 3.19 depicts a CMOS image sensor operating in direct integration. The two sources of current, $i_{p h}$ and $i_{d c}$, represent the photocurrent and dark current respectively.

Before the beginning of capture, the photodiode is reset. During exposure, the photocurrent is integrated onto the photodiode parasitic capacitor $C_{\text {diode }}$. The resultant charge $Q\left(t_{\text {int }}\right)$ (or voltage) is read out at the exposure time $t_{\text {int }}$. As indicated previously, dark current and additive noise are integrated along with the photocharge. This gives the overall 


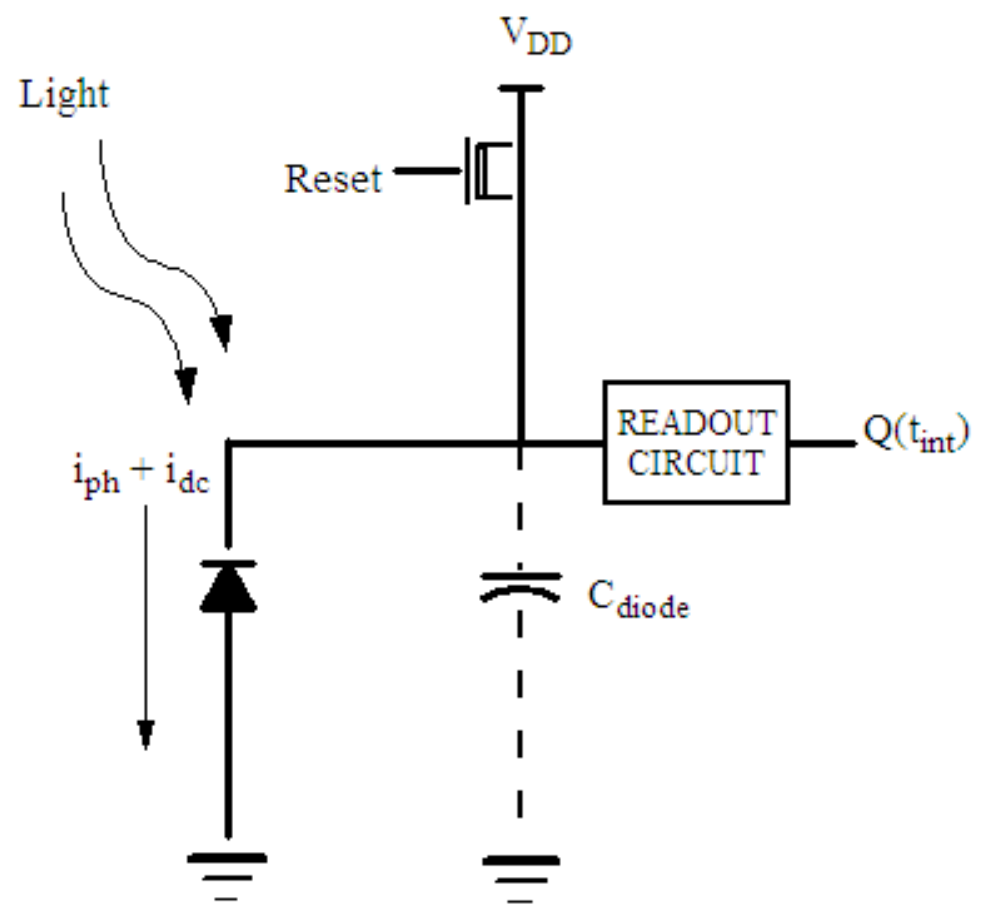

Figure 3.19: CMOS Image Sensor Pixel Model (after [65])

output charge $Q\left(t_{\text {int }}\right)$, expressed as

$$
Q\left(t_{i n t}\right)=\int_{0}^{t_{i n t}}\left(i_{p h}(t)+i_{d c}\right) d t+\text { noise }
$$

for $Q\left(t_{\text {int }}\right) \leq Q_{\text {sat }}$.

As described in Section 3.3.4, the temporal noise and FPN determine the range of illumination that can be detected by the sensor. They also determine the quality of signals produced within the detection range, measured by the signal-to-noise ratio (SNR).

Assuming CDS is employed to effectively cancel the offset FPN, the noise power at the end of integration can be expressed as the sum of four independent components: shot noise, read-out circuit noise, dark signal non-uniformity (DSNU), and gain FPN (PRNU) [58].

Denoting the integration time by $t_{\text {int }}$ and the charge of an electron by $q$, the average power of shot noise is $\frac{1}{q}\left(i_{p h}+i_{d c}\right) t_{i n t}\left[e^{-2}\right]$. Letting $\sigma_{\text {read }}^{2}, \sigma_{D S N U}^{2}$ and $\frac{1}{q^{2}}\left(\sigma_{P R N U} i_{p h} t_{i n t}\right)^{2}$ represent the noise power of the read noise, DSNU, and PRNU respectively, the SNR can be described by

$$
S N R=10 \log _{10} \frac{\left(i_{p h} t_{i n t}\right)^{2}}{\left(q\left(i_{p h}+i_{d c}\right) t_{i n t}+q^{2}\left(\sigma_{\text {read }}^{2}+\sigma_{D S N U}^{2}\right)+\left(\sigma_{P R N U} i_{p h} t_{i n t}\right)^{2}\right)} .
$$




\begin{tabular}{lcc} 
Parameter & $\begin{array}{c}\text { Current } \\
\text { Generation } \\
\text { of Pixels }\end{array}$ & $\begin{array}{c}\text { New Pixels with } \\
\text { Internal Reset }\end{array}$ \\
\hline \hline & & (2008) \\
Responsivity at FD* & $0.74 \mathrm{~V} / \mathrm{lux}-\mathrm{sec}$ & $1.17 \mathrm{~V} / \mathrm{lux}-\mathrm{sec}$ \\
Pixel Conversion Gain at FD & $85 \mu \mathrm{V} / \mathrm{e}^{-}$ & $152 \mu \mathrm{V} / \mathrm{e}^{-}$ \\
Pixel Dynamic Range & $65.9 \mathrm{~dB}$ & $72.6 \mathrm{~dB}$ \\
maximum SNR & $40.2 \mathrm{~dB}$ & $39.5 \mathrm{~dB}$ \\
Pixel Read Noise at Max Gain & $5.3 e^{-}$ & $2.1 e^{-}$ \\
Dark Current (at $55^{\circ}$ ) & 18 & 20 \\
PRNU at 50\% saturation & $0.95 \%$ & $0.80 \%$ \\
DSNU (at $35^{\circ}$ ) & $0.018 \%$ of & $0.02 \%$ of \\
& full-well & full-well \\
Pixel Capacity in Linear Range & $10500 e^{-}$ & $9000 e^{-}$ \\
\hline \hline
\end{tabular}

Table 3.2: Characteristics for Typical CMOS Pixels in Mobile Phones [66]

More information on the relationship between SNR and the dominating sources of noise for a typical CMOS sensor can be found at [58].

Using the simplified model developed above, Gamal and Eltoukhy showed that the dynamic range, given by $20 \log _{10}\left(i_{\max } / i_{\min }\right)$, can be expressed as

$$
D R=20 \log _{10} \frac{q \cdot Q_{w e l l}-i_{d c} \cdot t_{i n t}}{\sqrt{q i_{d c} t_{\text {int }}+q^{2}\left(\sigma_{\text {read }}^{2}+\sigma_{D S N U}^{2}\right)}}
$$

[58]. Unlike SNR, the dynamic range is reduced by increasing the integration time because of dark current.

Table 3.2 demonstrates the specifications for a 2 megapixel, 1/4 inch CMOS sensor used in mobile phone cameras. It includes specifications for the next generation version, introduced in 2008. 


\subsubsection{Colour Filter Imperfections and Cross-Talk}

In addition to physics of the CMOS sensor, the Bayer filter is an important factor, particularly if colour barcodes are to be considered. Filter imperfections lead to colour leakage between pixels. That is, each green filter in the Bayer array will inevitably admit some red and blue light (and likewise for the blue and red filters). This is discussed further in Section 3.4.3, and a typical Bayer response to light is illustrated in Figure 3.24. Although the camera processing unit attempts to correct filter imperfections (see Section 3.4.3), cross-talk is a significant source of degradation in the overall channel.

Overall, there are two sources of degradation associated with the Bayer filter: 1) filter imperfections / colour leakage and 2) the RGBG pattern down-sampling and demosaic reconstruction. These effects are particularly cumbersome for colour images, and therefore colour barcodes.

\subsubsection{The Overall CMOS Distortion Function}

The CMOS imager has been closely examined in order to properly model the distortion function, denoted by $D$ in the channel model of Equation 3.1. This function can be broken down into two-parts: 1) the decimation function related to the spatial resolution of the camera and 2) the light intensity response and tonal clipping related to the dynamic range of the sensor.

The CMOS decimation function can be modelled as $S \cdot U$, where $U$ is the image sensor PSF and $S$ is the down/up-sampling function. This can be interpreted as a low-pass filter followed by sampling.

Equation 3.38 allows for the calculation of the MTF corresponding to the point spread function $(U)$, denoted $U_{F}$. As seen in Section 3.3.2, the transfer function of the pixel array is a sinc function dependent on pixel pitch and size. To use Equation 3.38 for the twodimensional case, the product of the MTF in each direction is computed. For a square pixel shape, the MTF in both dimensions will be identical. Therefore, for a pixel pitch of $4 \times 4 \mu \mathrm{m}$ and a fill-factor of $50 \%$, the overall MTF is given by

$$
U_{F}(u, v)=\frac{1}{2} \cdot \operatorname{sinc}(\sqrt{2}(2 \pi u)) \cdot \operatorname{sinc}(\sqrt{2}(2 \pi v))
$$

and is plotted in Figure 3.20. 


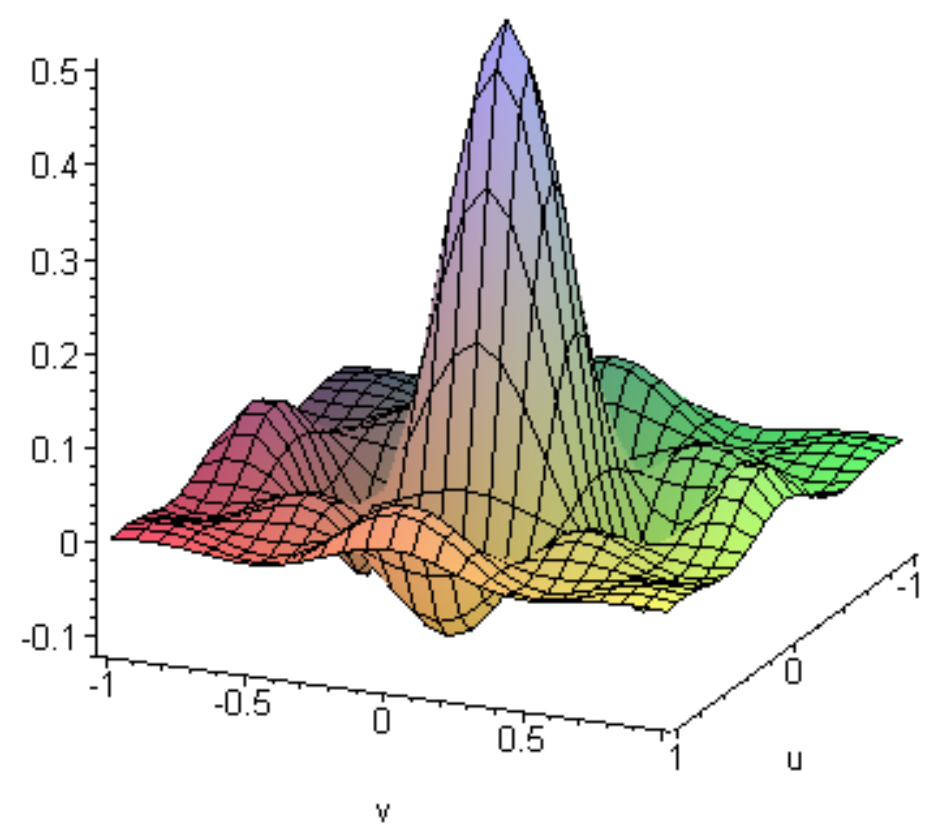

Figure 3.20: MTF of a Given CMOS Sensor Array

Because 1-megapixel resolution has become standard for cell phone cameras [67], and 2-megapixel is now very common, a $1280 \times 960$ pixel array, or even a $1600 \times 1200$ array, can be assumed for the model. Depending on the original resolution of the barcode, $S$ will upsample or down-sample the barcode image to the specified number of camera pixels using bicubic interpolation.

The decimation function described by $S \cdot U$ is only part of the problem. As described in Section 3.3.4, the CMOS image sensor also limits the dynamic range of the barcode image, particularly in low-light conditions. This should also be taken into account, particularly if the barcode design is to be grayscale (as opposed to binary). The sensor relationship is linear from the noise floor to the well capacity, with limits that depend on the camera and the light conditions. 
The imager response can be described mathematically by

$$
I_{\text {out }}(x, y)=\left\{\begin{array}{lll}
N_{\text {limit }} & \text { if } & I_{\text {in }}(x, y) \leq \frac{N_{\text {limit }}}{\text { gain }} \\
\left(I_{\text {in }}(x, y)\right) & \text { if } & \frac{N_{\text {limit }}}{\text { gain }}<I_{\text {in }}(x, y)<\frac{S_{\text {limit }}}{\text { gain }} \\
S_{\text {limit }} & \text { if } & I_{\text {in }}(x, y) \geq \frac{S_{\text {limit }}}{\text { gain }}
\end{array}\right.
$$

where parameters $N_{\text {limit }}, S_{\text {limit }}$, and gain can be adjusted to appropriately model the particular scenario.

Extending this to consider the colour response of the CMOS sensor, the model considers the red, green, and blue channels separately. Equation 3.48 incorporates colour cross-talk and breaks $I_{\text {out }}(x, y)$ into its three parts:

$$
\begin{aligned}
& I_{\text {out }_{R}}(x, y)=\left(R_{\text {in }} R\right) \cdot I_{i n_{R}}(x, y)+\left(G_{i n} R\right) \cdot I_{i n_{G}}(x, y)+\left(B_{i n} R\right) \cdot I_{i n_{B}}(x, y) \\
& I_{\text {out }_{G}}(x, y)=\left(R_{i n} G\right) \cdot I_{i n_{R}}(x, y)+\left(G_{i n} G\right) \cdot I_{i n_{G}}(x, y)+\left(B_{i n} G\right) \cdot I_{i n_{B}}(x, y) \\
& I_{\text {out }_{B}}(x, y)=\left(R_{i n} B\right) \cdot I_{i n_{R}}(x, y)+\left(G_{i n} B\right) \cdot I_{i n_{G}}(x, y)+\left(B_{\text {in }} B\right) \cdot I_{i n_{B}}(x, y)
\end{aligned}
$$

where $X_{i n} Y$ denotes the percentage of colour $X$ that passes through the colour $Y$ filters.

The PRNU should also be taken into account using the multiplicative zero-mean noise model of $I+n \cdot I$, where $n$ is a zero-mean uniformly distributed random noise. Additionally, for low-light conditions, a tiny proportion of random pixels may be mapped to white dots to model rapid saturation caused by an atypically high level of dark current in a small percentage of pixels [68]. This effect can be modelled by salt-and-pepper noise, which randomly assigns pixels to on or off depending on the degree of noise.

The CMOS sensor's influence over the image is intricate and somewhat unpredictable. There are many aspects of the physical reality that are difficult to model because of the variable factors across different cameras and different photographs. Regardless of what measures are taken to model and estimate the channel appropriately, the unpredictable chargeto-voltage relationship of CMOS cameras will continue to be problematic for wireless optical communication through digital photography.

For more information on CMOS image sensors, see [54, 58]. 


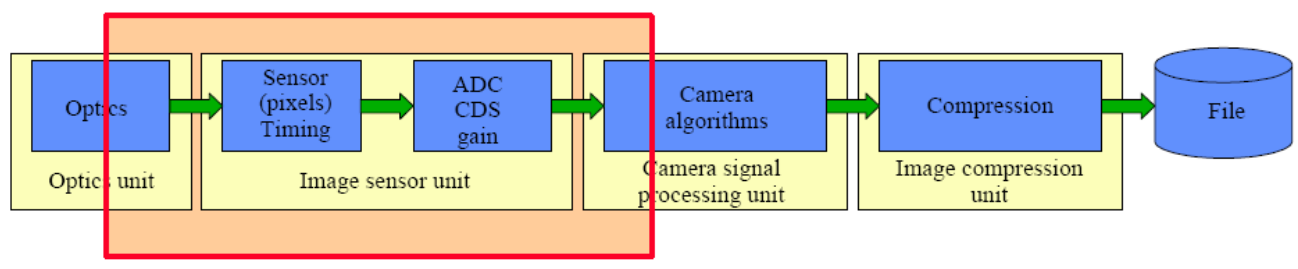

Figure 3.21: Scope of the SMIA Standard [69]

\subsection{Camera Processing}

As indicated in Section 3.3.4, the raw output of the CMOS sensor is not the actual output of the camera. As discussed previously, the demosaic process acts to interpolate the missing colour components of each RGB pixel. However, this is not the only processing applied to the raw sensor output.

The raw output is adjusted by a camera processing unit and then converted to JPEG format by a compression unit (see Figure 3.21). The processing/compression stage in the overall channel is of critical importance because the linear relationship between input intensity and output signal seen thus far is lost.

Because there are such a wide variety of camera phone solutions on the market, it is difficult to accurately model this component of the channel. Processing techniques will vary across manufacturers and across camera phone models, leading to a high degree of uncertainty.

In 2004, Nokia Corporation and ST Microelectronics developed the Standard Mobile Imaging Architecture (SMIA) to specify mobile camera housing, mechanical interconnection, functionality, register set and electrical interface. The SMIA standard is an open standard for use by all companies making, buying or specifying miniature integrated camera modules for use in mobile applications. The scope of SMIA is illustrated by the mobile camera phone block diagram of Figure 3.21.

Although the standard does not encompass many of the camera algorithms, nor the compression unit, colour-space compatibility and data bit representation must be compatible. Therefore, the camera processing and compression model that follows will adhere to SMIA specifications where applicable. More information on the SMIA standard can be obtained from [69]. 


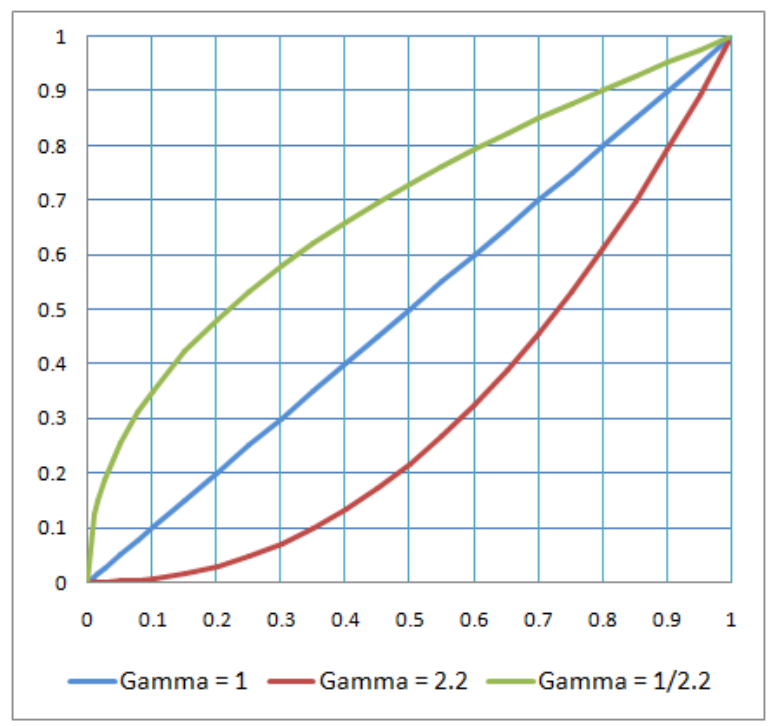

Figure 3.22: Gamma Curves

\subsubsection{Gamma Correction}

As discussed previously, imaging devices can exhibit a nonlinear relationship between input intensity and the output signal (see Table 3.4.1). This is not only true of imaging devices, but of displays. LCD and CRT displays exhibit a nonlinear signal-to-light-intensity response described by the red curve in Figure 3.22, where the gamma exponent is greater than one.

All CRT displays have a power-law transfer characteristic with a gamma exponent of approximately 2.2. By convention, input and outputs are scaled from 0 to $1(0=$ black and 1 = white) so that the power function can be described entirely by the gamma exponent.

Because the CRT gamma is fixed at around 2.2, to achieve an end-to-end exponent closer to 1 , one exponent in the image pipeline must act as a nonlinear complement (that is, with a gamma of 1/2.2). Generally, this complementing process is termed gamma correction.

In all broadcast video systems, gamma correction is done in the camera. This standardization was chosen because the required processing in a few studio television cameras is much less expensive than in the large number of broadcast receivers.

For digital video signals, which are stored in computer frame buffers, the gamma correction is sometimes applied before the buffer and is sometimes applied afterward with a look-up table (LUT). Complications can arise when images are exchanged and differences in gamma-correction conventions cause images to appear much too bright and washed-out, 
or too dark and contrasted.

Interestingly, the human eye's perception of light is not a linear function of intensity. In fact, the eye's response is similar to that of a gamma correction curve (see Figure 3.4.1). Therefore, if gamma correction is applied to the sensor response before it is quantized to an integer for storage, fewer bits are required to store the image [70].

With gamma-correction applied to the raw image, 8 bits/colour is sufficient to represent the photograph compared to 12 bits/colour without gamma-correction. In comparison to a linearly encoded image, fewer samples are allocated to brighter parts of the tonal range and more samples are allocated to the darker portions of the tonal range. The 256 intensity values are assigned in a manner that approximates how the intensity changes are perceived by the human eye.

As will be seen, mobile phone cameras automatically compress all photographs using the JPEG lossy compression standard. For this reason, and in light of the visual considerations for CRT/LCD displays, a gamma-correction curve is applied to the raw sensor output $[69,70,71]$. So although the sensor output is a linear function of light intensity input, the camera actually exhibits a non-linear response to light.

The International Electrotechnical Commission (IEC) 61966 standard defines the sRGB colour space, which allows for direct display on typical monitors. The sRGB transformation was designed to approximate a gamma of about 2.2, but with a linear portion near zero (black) to avoid an infinite slope. The standard is followed by LCDs, digital cameras, printers, and scanners. In the SMIA specification, gamma-correction of the raw output complies with the IEC sRGB colour space. For more information, see [72, 73, 74, 75].

Although the sRGB intensity versus colour value response has a linear region and a region with a gamma of approximately 2.4 , the overall curve is very close to that of a gamma curve of 2.2. Therefore, the gamma exponent of the mobile phone is assumed to be $1 / 2.2$ for the $\mathrm{R}, \mathrm{G}$ and $\mathrm{B}$ channels for consistency with SMIA.

\subsubsection{Demosaicing}

An important consideration in the colour response of the camera is the colour under-sampling applied by the RGBG Bayer filter. The demosaic process has the effect of blurring colour, particularly in the red and blue domains. 


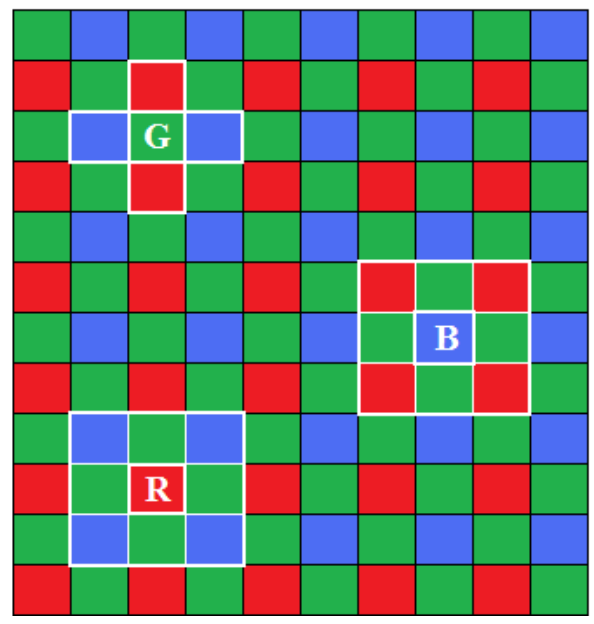

Figure 3.23: Demosaicing: Nearest Neighbours for each Bayer Filter Colour

The demosaic process was described previously as bilinear interpolation. Because the Bayer filter has a RGB ratio of 1:2:1, the nearest-neighbour configuration is different for green pixels than it is for blue and red. This can readily be observed from Figure 3.23.

The bilinear interpolation process is very simple. For red/blue pixels, the average of the four neighbouring green pixels is taken as the $G$ value, and the average of the four neighbouring blue and red pixels are taken as the $B$ and $R$ values respectively.

For green pixels, because there are only two nearest neighbours for both red and blue, the average is taken over two pixels instead of four. The result is generally less accurate along either the horizontal or vertical axis (depending on the layout of red and blue pixels surrounding the green).

It should be noted that some digital cameras use a more complex demosaic method. However, the simplicity of bilinear interpolation tends to make it more appropriate for mobile phones. Although some phones may use a more sophisticated interpolating algorithm, bilinear interpolation will be assumed.

Because demosaicing consists of averaging neighbouring pixels, the overall result is spatial blurring of the image. The Bayer filter imager essentially under-samples the scene in three colour domains. The variance of blur will be higher in the red and blue domains because the image is under-sampled by a factor of four, whereas the green domain of the image is under-sampled by a factor of two.

Overall, image information in the green domain is preserved to a greater degree of accu- 
racy than information in the red and blue colour domains. This characteristic must be taken into account if colour barcodes are to be considered.

\subsubsection{Colour Correction and Other Processing}

Many digital cameras apply other image processing techniques to the raw sensor output. The processing applied in a mobile phone is expected to be minimal. However, with cell phone cameras becoming more sophisticated, it would not be surprising for some image processing techniques to be employed. For instance, it would be a reasonable assumption that the camera performs some degree of colour-correction. In some camera phones, a contrast-stretching curve may also be applied to the image before compression. Automatic White Balance (AWB) is another common image processing technique used in mobile phone cameras.

To see why processing the raw image is beneficial, consider the colour-mixing that occurs as a consequence of the Bayer filter. For instance, each green pixel filter will inevitably admit some red light and blue light. The same is true of red and blue pixels. However, the red light leaking into blue pixels and the blue light leaking into red pixels is less significant due to the spectral response of Bayer filter, which is a function of wavelength. The spectral response of some typical Bayer filters can be found at [76, 77, 78]. Figure 3.24, taken from [76], illustrates the response for a particular Bayer filter. It should be noted however, than the Bayer filter colour responses vary and that this example does not accurately represent all camera sensors.

Cameras may aim to correct for the overlap observed in Figure 3.24 through some processing of the raw image. The SMIA specifies a standard colour space to generate common data from different SMIA sensors. The SMIA colour matrix is described by

$$
\left[\begin{array}{ccc}
\text { RedInRed } & \text { GrnInRed } & \text { BluInRed } \\
\text { RedInGrn } & \text { GrnInGrn } & \text { BluInGrn } \\
\text { RedInBlu } & \text { GrnInBlu } & \text { BluInBlu}
\end{array}\right]
$$

where $x$ In $y$ specifies the recommended amount of the sensor $x$ signal to be in the output $y$ signal ( $x, y \in\{\operatorname{Red}, G r n, B l u\})$. (The colour matrix values are sensor-specific.)

Once the image is converted to the colour-corrected RGB format, it is then converted to Luminance, Chrominance Blue, Chrominance Red $\left(Y C_{B} C_{R}\right)$ representation. 


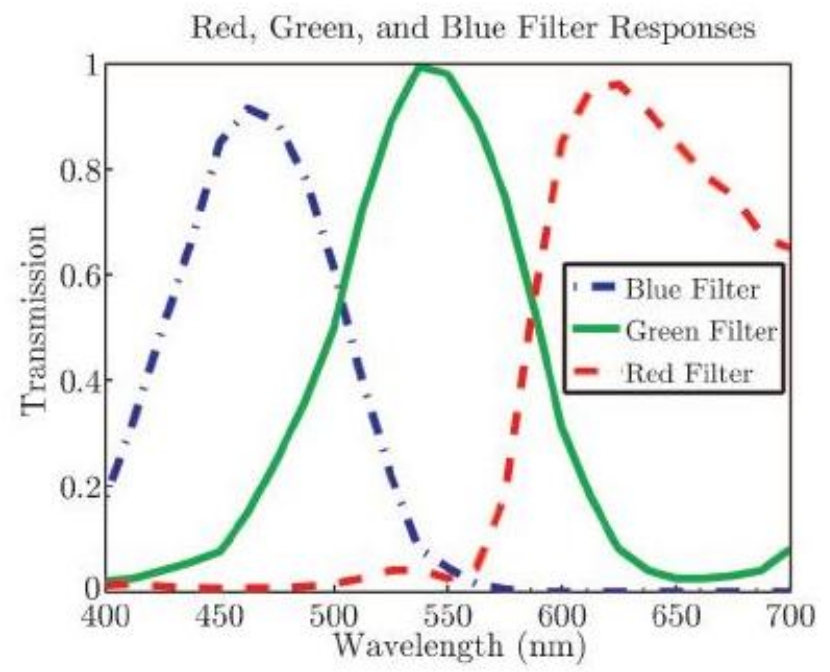

Figure 3.24: Spectral Response of a Typical Bayer Filter [76]

Because the eye is more sensitive to luminance, using the $Y C_{B} C_{R}$ representation of the image instead of RGB allows for the $C_{B}$ and $C_{R}$ domains to be down-sampled with little image degradation. The conversion from 8-bit digital gamma-corrected RGB to $Y C_{B} C_{R}$, also known as $Y U V$, is described by

$$
\begin{aligned}
& Y=0.299 \cdot \mathrm{R}+0.587 \cdot \mathrm{G}+0.114 \cdot \mathrm{B} \\
& C_{B}=-0.1687 \cdot \mathrm{R}-0.3313 \cdot \mathrm{G}+0.5 \cdot \mathrm{B}+128 \\
& C_{R}=0.5 \cdot \mathrm{R}-0.4187 \cdot \mathrm{G}-0.0813 \cdot \mathrm{B}+128,
\end{aligned}
$$

rounded to the nearest integer.

This luminance/chrominance representation is as specified by SMIA, where YUV is the 8-bit gamma-corrected $Y C_{B} C_{R}$ colourspace defined by the ITU-R BT601.4 standard. The $Y$, $C_{B}$, and $C_{R}$ values are integers in between 0 and 255. This is the colourspace that is used by the JPEG file interchange format, where the $C_{B}$ and $C_{R}$ are down-sampled by a factor of 2 in both directions. For more information, see [72, 79, 80, 81, 82].

\subsection{Additive Noise}

In the channel model described mathematically by Equation 3.1, $n(i, j)$ represents the additive noise. In addition to the distortions previously discussed, atmospheric, environmental, 
and device variations and imperfections will inevitably lead to the addition of noise to the image.

Additive White Gaussian Noise (AWGN), independent of RGB values, is typically assumed in the modelling of digital imaging system [83, 84]. Although the AWGN model does not describe the noise distribution in an exact manner, it provides a simple and natural model to work with. If the barcode is designed to overcome AWGN, it will generally be robust to any other form of additive noise imposed by the channel.

A stochastic process is considered white if it has a constant spectral density, and Gaussian if it exhibits a normal distribution. The two-dimensional AWGN applied to the image is circularly symmetric and is defined by its variance, $\sigma^{2}=\sigma_{x}^{2}=\sigma_{y}^{2}$.

\subsection{JPEG Compression}

Cellular phones do not have much storage and therefore must be judicious with memory allocation. Digital photographs on cellular phones are automatically compressed using the JPEG compression standard.

JPEG, which stands for Joint Photographers Expert Group, is a lossy baseline coding system based on the discrete cosine transform (DCT). It has a low-pass characteristic (like the other elements of distortion seen in the channel model in Equation 3.1) and is the final distortion applied to the barcode in the imaging process. JPEG compression takes advantage of the fact that the human visual system is not as sensitive to higher frequencies by discarding many of the high frequency details in the image.

To understand JPEG, an overview of the DCT is helpful. The DCT is an orthogonal transform similar to transformations to the frequency domain. It is, in fact, equivalent to the discrete Fourier transform (DFT) of a symmetrisized extension of the input set of samples. There are algorithms available to efficiently compute the DCT [85].

The DCT is specified by the $(i, k)^{t h}$ element, $d_{i k}$, of the $N \times N$ DCT coefficient matrix, D. These elements are expressed as

$$
d_{i k}= \begin{cases}\sqrt{\frac{2}{N}} \cos \left(\frac{\pi}{N} i\left(k+\frac{1}{2}\right)\right) & i=1,2, \ldots, N-1 \\ \sqrt{\frac{1}{N}} \cos \left(\frac{\pi}{N} i\left(k+\frac{1}{2}\right)\right) & i=0\end{cases}
$$

where $i$ and $k$ are indexed over the range $0,1, \ldots, N-1$ [85]. 
Because digital images are already organized into a two-dimensional array, the DCT is convenient (and very efficient given the availability of fast DCT algorithms, similar to the Fast Fourier Transform (FFT)). The DCT representation of an $M \times N$ image matrix $I$ is obtained through matrix multiplication with DCT coefficient matrices:

$$
I_{D C T}=D_{M \times M} \cdot I \cdot D_{N \times N}^{T}
$$

Because the DCT is an orthogonal transformation, the inverse of the transform matrix is simply the matrix transpose. Therefore, the image can be fully recovered using the transpose of the transform matrices,

$$
I=D_{M \times M}^{T} \cdot I_{D C T} \cdot D_{N \times N}
$$

The representation of the $M \times N$ image $f(x, y)$ image in the DCT frequency domain is described by

$$
T(u, v)=\sum_{x=0}^{M-1} \sum_{y=0}^{N-1} f(x, y) \alpha_{M}(u) \alpha_{N}(v) \cos \left(\frac{\pi}{M}\left(x+\frac{1}{2}\right) u\right) \cos \left(\frac{\pi}{N}\left(y+\frac{1}{2}\right) v\right),
$$

where

$$
\alpha_{P}(w)= \begin{cases}\sqrt{\frac{1}{P}} & w=0 \\ \sqrt{\frac{2}{P}} & w=1,2, \cdots, P-1 .\end{cases}
$$

The image $f(x, y)$ can then be expressed as a function of its 2-D DCT, $T(u, v)$,

$$
\mathbf{F}=\sum_{u=0}^{M-1} \sum_{v=0}^{N-1} T(u, v) \mathbf{H}_{\mathbf{u v}}
$$

where $\mathbf{F}$ is an $M \times N$ matrix containing the pixels of $f(x, y)$ and the $\mathbf{H}_{\mathbf{u v}}$ are the DCT basis images/functions.

It can be observed that the DCT corresponds closely to the DFT, but in the real domain. This makes it an appealing choice for the transform encoding of digital images. For more information on the DCT, see [85].

JPEG defines different coding systems, not all of which are lossy. The baseline coding system is the aforementioned lossy system based on the DCT and most commonly used. In the baseline system, the input and output data is limited to 8 bits, and the quantized DCT values are restricted to 11 bits. 
JPEG is performed on $Y C_{B} C_{R}$ images, as opposed to $R G B$, to allow for a greater degree of compression. As discussed in Section 3.4.3, the $C_{B}$ and $C_{R}$ components of the image are down-sampled. The JPEG standard specifies a down-sampling factor of 2 in both directions for both chrominances [82].

Once the appropriately down-sampled $Y C_{B} C_{R}$ representation of the image is obtained, the JPEG compression is performed in three consecutive steps: 1) DCT computation, 2) normalization/quantization, and 3) variable-length coding assignment.

Firstly, the image is divided into $8 \times 8$ pixel sub-images. As each sub-image is encountered by the processor, the 64 pixels are level-shifted by 128 (i.e. mapped from [0, 255] to $[-128,127])$. The sub-image processing is done from left to right, top to bottom. The 2-D DCT of the sub-image is computed (see Equation 3.50), yielding $T_{s u b}(u, v)$. The values are then quantized.

Quantization is done by applying a threshold that varies as a function of the location of each coefficient within each sub-image. Recall from Equation 3.54 that an image can be expressed as a function of its 2-D DCT. To eliminate the basis images that make the smallest contribution to the total sum, a masking function $\gamma(u, v)$ is defined as

$$
\gamma(u, v)= \begin{cases}0 & \text { if } T_{\text {sub }}(u, v) \text { satisfies a specified truncation criterion } \\ 1 & \text { otherwise }\end{cases}
$$

and then $\mathbf{F}_{\text {sub }}$, the representation of sub-image $f_{\text {sub }}(x, y)$, is specified by

$$
\mathbf{F}_{\text {sub }}=\sum_{u=0}^{N-1} \sum_{v=0}^{N-1} \gamma(u, v) T_{s u b}(u, v) \mathbf{H}_{\mathbf{u v}} .
$$

This process is called thresholding. JPEG combines thresholding and quantization into one step by replacing $\gamma(u, v) T_{\text {sub }}(u, v)$ in Equation 3.56 by

$$
\widehat{T}_{\text {sub }}(u, v)=\text { round }\left[\frac{T_{s u b}(u, v)}{Z(u, v)}\right],
$$

where $Z(u, v)$ is an element of a predefined transform-normalization array.

If $|Z(u, v)|>2 T_{\text {sub }}(u, v)$, then $\widehat{T}_{s u b}(u, v)$ is assigned a value of zero, completely discarding that particular transform coefficient. $\widehat{T}_{s u b}(u, v)$ is represented by a variable-length code that increases in length as the magnitude of $\widehat{T}_{s u b}(u, v)$ increases. Therefore, the number of bits used to represent $\widehat{T}_{s u b}(u, v)$ is determined by $Z(u, v)$, allowing for many compression levels. 


\begin{tabular}{|l|l|l|l|l|l|l|l|}
\hline 16 & 11 & 10 & 16 & 24 & 40 & 51 & 61 \\
\hline 12 & 12 & 14 & 19 & 26 & 58 & 60 & 55 \\
\hline 14 & 13 & 16 & 24 & 40 & 57 & 69 & 56 \\
\hline 14 & 17 & 22 & 29 & 51 & 87 & 80 & 62 \\
\hline 18 & 22 & 37 & 56 & 68 & 109 & 103 & 77 \\
\hline 24 & 35 & 55 & 64 & 81 & 104 & 113 & 92 \\
\hline 49 & 64 & 78 & 87 & 103 & 121 & 120 & 101 \\
\hline 72 & 92 & 95 & 98 & 112 & 100 & 103 & 99 \\
\hline
\end{tabular}

Figure 3.25: Typical JPEG Normalization Array, $Z(u, v)$

The array displayed by Figure 3.25 is a normalization array that has been widely used in the JPEG standardization. It weighs each coefficient of a transformed sub-image according to heuristically determined perceptual or psychovisual importance [50].

Once the DCT coefficients for each sub-block have been quantized, the elements of $\widehat{T}_{s u b}(u, v)$ are recorded in accordance with the JPEG zigzag pattern. Essentially, they are arranged in a one-dimensional matrix according to increasing spatial frequency. This allows the encoder to take advantage of a long run of zeros that typically results from the reordering.

The DC coefficient, $\widehat{T}_{s u b}(0,0)$, is difference coded relative to the DC coefficient of the previous sub-image. The nonzero, non-DC coefficients (that is, all $\widehat{T}_{\text {sub }}(u, v) \neq 0$ except $\left.\widehat{T}_{s u b}(0,0)\right)$ are coded using a variable-length code that defines the coefficient value and the number of preceding zeros. Huffman coding tables are provided by the JPEG standard.

To adjust the compression ratio, the normalization array $Z(u, v)$ is scaled. Typical cell phones allow a user to choose a compression quality. For example, the user can set the image quality settings to basic, normal, or high on the Nokia 6085 cell phone. It is important to note that the JPEG normalization array differs between nearly all digital cameras and software packages. Camera manufacturers independently choose an arbitrary image quality name (or level) to assign to their $8 \times 8$ normalization matrix. Therefore, the names cannot be compared between makes, and often even between models by the same manufacturer [86].

Because users may wish to store many images on their cell phone, lower quality settings 
may be desired. However, barcode photographs should be compressed using the highest available JPEG quality in order to reduce the probability of decoding failure. This should not be of great concern to the average consumer since most users will have no reason to keep a barcode photograph once they have decoded the information stored in it.

\subsection{Transmitter Nonlinearities}

The mathematical channel model of Equation 3.1 does not include any distortion imposed by the transmitter. This is an accurate model for the case where the barcode is displayed on paper. However, if the barcode appears on an LCD or CRT, the display device will distort the barcode image prior to the photographing process.

Display devices such as television and computer CRTs, as well as LCDs, do not exhibit a linear relationship between input and output intensities. The gamma parameter described in Sections 3.3.4 is greater than 1 for both devices. The result is a noticeably impaired colour representation of the picture on the display. The gamma distortion must be taken into account in the channel model since computer screens are considered to be potential transmitters of the barcode image.

As previously outlined, CRT displays generally exhibit a non-linear relationship between input values and output intensities, with a gamma of approximately 2.2. All computer displays typically aim to realize an sRGB colour space, as defined by the IEC 61966 standard. The sRGB colour space closely resembles the 2.2 gamma curve of traditional CRT displays.

Because the CRT gamma curve is not inherent of LCDs, the display may be in a state which differs from sRGB. Nearly all LCDs require accurate adjustment of hardware settings, as well as calibration and strong adjustments in the graphics card, in order to be able to accurately display sRGB graphics and images. Without proper calibration, colours appear incorrectly on the screen. This characteristic has consequences for the overall channel model. For instance, if the transmitter nonlinearity is unknown, the barcode being displayed cannot be accurately pre-distorted.

In general, the sRGB colour space is assumed for CRT and LCD displays, but there is a degree of variance for transmitter distortion due to calibration uncertainty. For more infor- 
mation, see $[56,73,74,75,87]$.

\subsection{Areas of Uncertainty}

The camera processing uncertainty, combined with the other variables involved in the CMOS imager response, make channel modelling an intricate task.

First, the noise floor and well capacity are dependent on the individual CMOS sensor and on the lighting conditions when the photograph is taken. Therefore, the dynamic range varies from one camera phone to the next, and from one photograph to the next. The three variables in the CMOS nonlinearity are the noise floor, the full-well capacity, and the pixelconversion gain (expressed in $\mu V / e^{-}$).

Second, camera colour-response/correction varies across cameras. This is in addition to a degree of uncertainty regarding any other raw image processing performed by the camera.

For effective communication, estimation of the sensor response should be made at the transmitter, or at the receiver through the use of pilot signals in the barcode. In order to reduce the complexity of the estimation process, mobile phone users could potentially calibrate their phones ahead of time using specified barcodes.

By snapping a photo of specified calibration barcode under the specified lighting conditions, the phone's barcode decoding software would have the phone's response to light a priori. The same could be done to determine the overall camera response to colour. This training process would significantly reduce the number of variables at the decoder, though lighting conditions will remain unknown.

\subsection{Preliminary Tests}

It was hypothesized that a (spatial) discrete multi-tone (SDMT) approach to modulation, such as that used for the Granite Code symbology, would provide for the highest datadensity based on the low-pass characteristic of camera imaging channels. SDMT modulation has been studied for the pixel-matched optical channel, yielding very high data densities [88]. This technique, employed for Granite Codes using the Fourier domain, is superior to spatial domain techniques under orthographic projection and no camera processing, as discussed in Section 2.2.6 and Section 2.3. 
A preliminary research objective was to evaluate the feasibility of a SDMT approach to barcode design under a camera phone channel. A Motorola cell phone, equipped with a 2 megapixel camera, was used for some preliminary experimentation. The specifications for the phone can be found in Appendix B. The camera phone was used to determine whether to pursue the SDMT approach or to abandon it for another method.

\subsubsection{Setup}

A Granite-like square barcode was used for preliminary tests (see Figure 3.26). Two different transmitters were used, an LCD monitor and paper, with the Motorola camera phone as the receiver. Bits were encoded such that each of the lower frequency bins took on values of ${ }_{-} 1_{-}^{+} j$. To ensure that a real image was produced, complex symmetry was imposed in the Fourier domain. No pre-processing, noise-shaping, water-filling, or other processing techniques used by S. Gupta to create a true Granite Code [33] were employed.

Several test photographs were taken of various barcode images, first using paper, then an LCD monitor, in a well-lit room. The photographs were loaded onto a computer to be evaluated against the original barcode in MATLAB (the barcode was not processed on the phone itself). After being manually cropped according to a 'visual acquisition', the barcode was digitally resized. No perspective correction was performed. The values in the frequency bins were compared to the original values, and the errors (i.e. flipped bits) were counted.

Using a $400 \times 400$ pixel barcode with bits in only the lowest $151 \times 151$ frequency bins (for a data density of 0.1425 bits/pixel), typical binary error rates were $7-8 \%$ and $16-20 \%$ using paper and the LCD respectively. Given that no processing or coding was performed (a simple slicer at the receiver made all the hard decisions), this was an encouraging result. Perhaps more significant was that less than $4 \%$ of errors occurred in the lower third of frequency bins, and more than $85 \%$ of errors occurred in the highest third. This outcome supported the notion of storing information in low frequency regions of a barcode image.

Following promising results with the simple black and white OFDM barcode, it was determined that an SDMT approach warranted further investigation. 


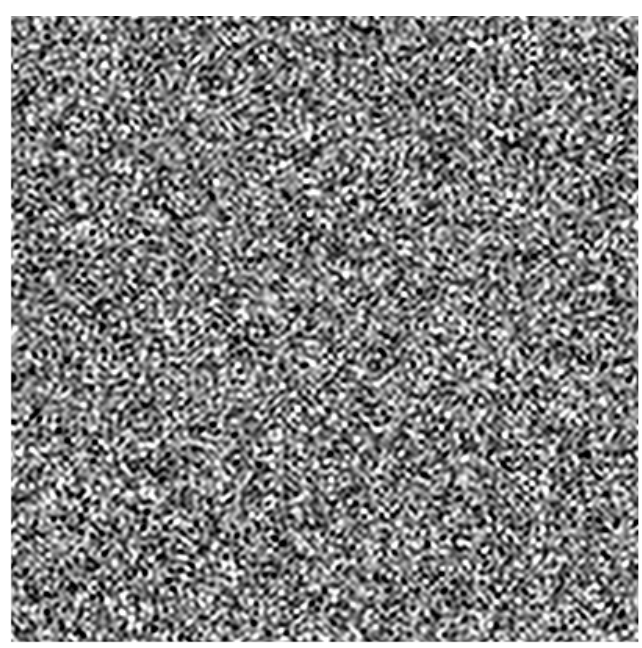

Figure 3.26: Granite-like, OFDM Barcode used in Preliminary Tests

\subsubsection{Black and White versus Colour}

It was originally hypothesized that a colour barcode would not be feasible for an SDMT barcode due to the relatively poor colour response of camera phones and, more importantly, the inevitable colour cross-talk. These impediments are particularly problematic for an approach like discrete multi-tone modulation, in which three independent images that require the full range of intensities in $[0,255]$ are overlaid to create an RGB image.

Without significant colour processing, the cross-talk and nonlinearities may negate any increase in density allowed by tripling the number of channels. To determine whether this was the case, some colour barcodes were examined using the simple setup of Section 3.9.1.

While the number of errors per colour channel increased, the increase was not nearly enough to overcome the gain in data density resulting from the two additional channels. For example, a typical trial using the LCD transmitter showed an increase from $18 \%$ error to $21 \%$ error per channel versus a threefold increase in data density. This was a remarkable preliminary result, particularly because no processing was being performed on the acquired barcode. This result initiated the RGB barcode design (see Figure 3.27).

\subsubsection{DFT versus DCT}

From a communications standpoint, the natural choice for a basis in a DMT system may be the Fourier domain as it is central to our understanding and design of RF communication 


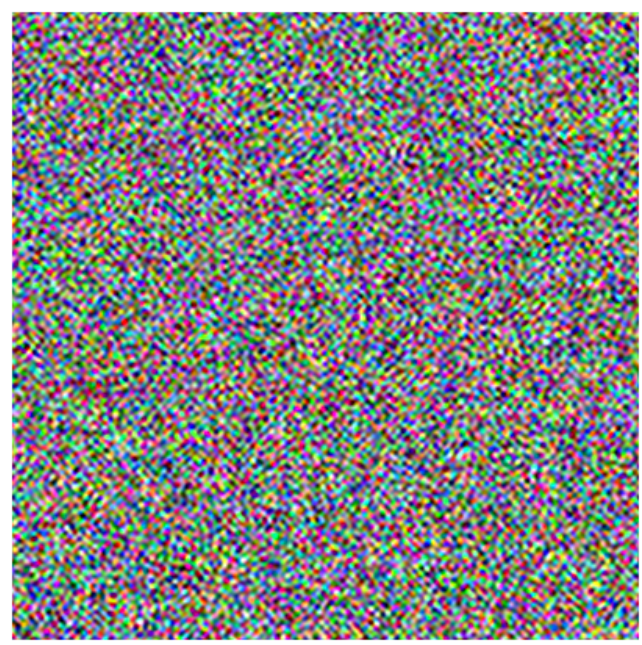

Figure 3.27: RGB version of Barcode used in Preliminary Tests (Figure 3.26)

systems. For a barcode, the Fourier domain remains an intuitive choice due to the Fourier transforming property of a lens (Section 3.2.2). However, there are other basis function choices for an SDMT design worth considering.

The JPEG standard makes use of the discrete cosine transform (DCT), as discussed in Section 3.6. The DCT was chosen for the JPEG standard primarily for its de-correlation and energy compaction characteristics. JPEG compression is an inevitable channel characteristic for mobile phone cameras. Therefore, because compression is performed according to information in the discrete cosine domain, a barcode encoded in the DCT domain was tested.

Although a DCT barcode can only encode a real value in each frequency bin, it does not require complex symmetry. Therefore, the same number of bits can be encoded in a DCT barcode as in a DFT barcode. Bits were encoded such that each of the lower frequency bins took on values of ${ }_{-}^{+}$. The RGB format was maintained following the results of previous tests (Section 3.9.2). A DCT barcode is displayed in Figure 3.28.

Using the setup of Section 3.9.1, the DCT RGB version outperformed the DFT RGB version (the same results were produced by a black and white test case). To support this result, the barcodes were compressed without any other components of the channel being imposed. Under a mildly aggressive JPEG compression in MATLAB (75\% quality), the DCT barcode was far more robust than the DFT barcode. Under a 90\% quality JPEG compression, similar to the level used in the Motorola phone, the DCT barcode still held a significant 


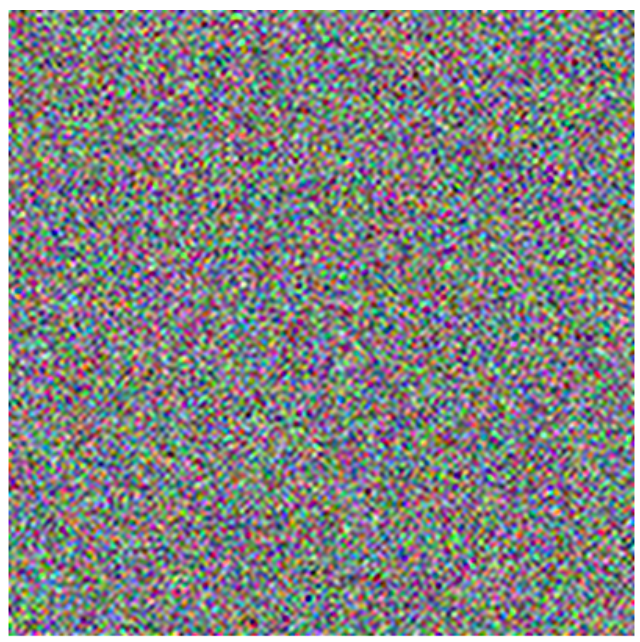

Figure 3.28: A DCT version of the RGB barcode

advantage over the DFT barcode.

Further work may eventually uncover a better waveform choice than the DCT, particularly because the new JPEG standard, JPEG2000, uses the Wavelet Transform (WT) as opposed to the DCT. JPEG2000 may eventually replace the current compression standard used in mobile phones [89]. However, seeking an optimal waveform was out of the scope of this thesis, and the DCT was chosen as the barcode waveform.

It was also determined throughout the preliminary testing that an LCD display was a reasonable transmitter choice and would therefore be studied as the primary means of transmission. It is expected that any results will also apply to paper and that paper transmissions will yield higher performance holding all other factors equal. 


\section{Chapter 4}

\section{MATLAB Channel Simulator}

In order to develop and optimize a barcode for a particular channel, a simulation environment is desirable. Instead of administering field tests similar to those described in Section 3.9 to assess performance and adjust the barcode design, simulations allow for a more iterative and efficient design and optimization process. Field tests will ultimately determine the success of a barcode, but only after a significant amount of adjusting and optimizing has been performed to design the barcode appropriately.

MATLAB, which stands for Matrix Laboratory, is a high-performance array-oriented language for technical computing. It is complemented by application-specific solutions called toolboxes, one of which is the Image Processing Toolbox. Because MATLAB aims to facilitate matrix calculations and offers a wide range of image-specific functions, it is a natural choice for the simulation environment.

To achieve the desired channel simulation, a MATLAB function was developed. It accepts a barcode (or other) image as input and carries out each of the distortion functions described in Chapter 3 before adding Gaussian noise to the image. The output of the MAT$\mathrm{LAB}$ function is a distorted and noisy version of the image (in RGB format).

All stages of the simulator store the image in unsigned 8-bit integer (uint8) RGB format unless otherwise specified. This means all three colours per pixel take on integer values in the range $[0,255]$. If the input image is of a different format, it is converted to uint8 RGB before simulation commences. Input images should exhibit 4:3 aspect ratios for a 4:3 aspect ratio output. 


\subsection{MATLAB Transmitter Nonlinearity}

The first distortion applied to the input image is a gamma function that models the effect of an LCD display as described in Section 3.7. If the model were to describe the channel for a printed barcode, this function would be omitted. The Matlab imadjust function is used with the output dynamic range set to equal the input dynamic range (see [90] for more information). The gamma value has a default of 2.2, but can be adjusted to model any LCD accordingly.

\subsection{MATLAB Warping Function}

As explained in Section 3.1, the warping applied to the barcode by the camera orientation is described by the concatenation of translation, rotation, and perspective projection transformations. Therefore, the MATLAB warping function requires the angle of rotation, tilt, and pan as inputs. In general, the relative distance between photographer and barcode is not variable, nor is the focal length of the camera. Instead, the warping function assumes the built-in Image Processing Toolbox camera projection distances by default (though these distances can easily be modified). This narrows the number of variables and the scope of the model to provide for a simpler, more easily operated simulation environment. The size of the barcode with respect to background is also variable and the background is set to be white.

The warping function first describes the two-dimensional image as a plane in threedimensions. The intensity at each coordinate is described by the three RGB colour values of the image. To apply the rotation and warping, the translation matrix, $T$, that maps the center of the image plane to the origin, the tilt, pan and rotation matrices seen in Section 3.1, $R_{\beta}, R_{\lambda}$ and $R_{\alpha}$ respectively, and the translation matrix, $B$, that maps the image back to its original center are combined to form the transformation matrix $W$,

$$
W=B \cdot R_{\beta} \cdot R_{\lambda} \cdot R_{\alpha} \cdot T
$$

and applied to the image plane.

The background lighting is an optional setting that is incorporated into the warping function. This is done using the Matlab camera lighting controls. It is applied at this point 
because the image is already being mapped to a Matlab plane, simplifying the task of adjusting the gain of the individual pixels. External light is a variable between 0 and 1, where 1 has no effect on the image, and 0 produces a completely black image (i.e. zero models a photograph taken in a completely unlit room).

Once the warping is performed, the MATLAB camera perspective projection is applied to the plane in three-dimensions, resulting in the final 2-D warped image.

\subsection{MATLAB Lens PSF Function}

To apply the transfer function developed in Section 3.2 to the image, linear spatial filtering, or spatial convolution, is performed. This is accomplished by multiplying each pixel in a given neighbourhood by a coefficient and summing the results to obtain the response at each point $(x, y)$. Therefore, an $a \times b$ neighbourhood requires $a \cdot b$ coefficients, which are organized in an $a \times b$ matrix called a filter mask or kernel.

The linear spatial filtering process is very similar to the filtering of electrical signals. Once the mask is defined, it is rotated by $180^{\circ}$ in order to perform convolution (and not correlation). The filter mask center is moved across the image in order to calculate the response at each pixel, as illustrated by Figure 4.1.

The PSF of the lens, as seen in Section 3.2, is influenced by the degree of defocus and motion. In fact, once these effects have been taken into account, they override the lens PSF completely. To model this effect in MATLAB, a Gaussian filter mask is applied to the image, followed by a 'motion' mask. If no motion blur is desired for simulation, it is omitted.

The Gaussian filter is obtained through the Gaussian probability distribution function. For instance, for a $3 \times 3$ mask, the center pixel has the highest coefficient (it is weighted the greatest). The four vertical/horizontal pixel neighbours are weighted equally, but less, and the four diagonal neighbours are weighted the least. The degree of weighting applied to the neighbours depends on the variance. For higher variances, a larger mask is generally used $(5 \times 5,7 \times 7$, etc. $)$ to account for the greater roll-off of the distribution. A $3 \times 3$ Gaussian filter 


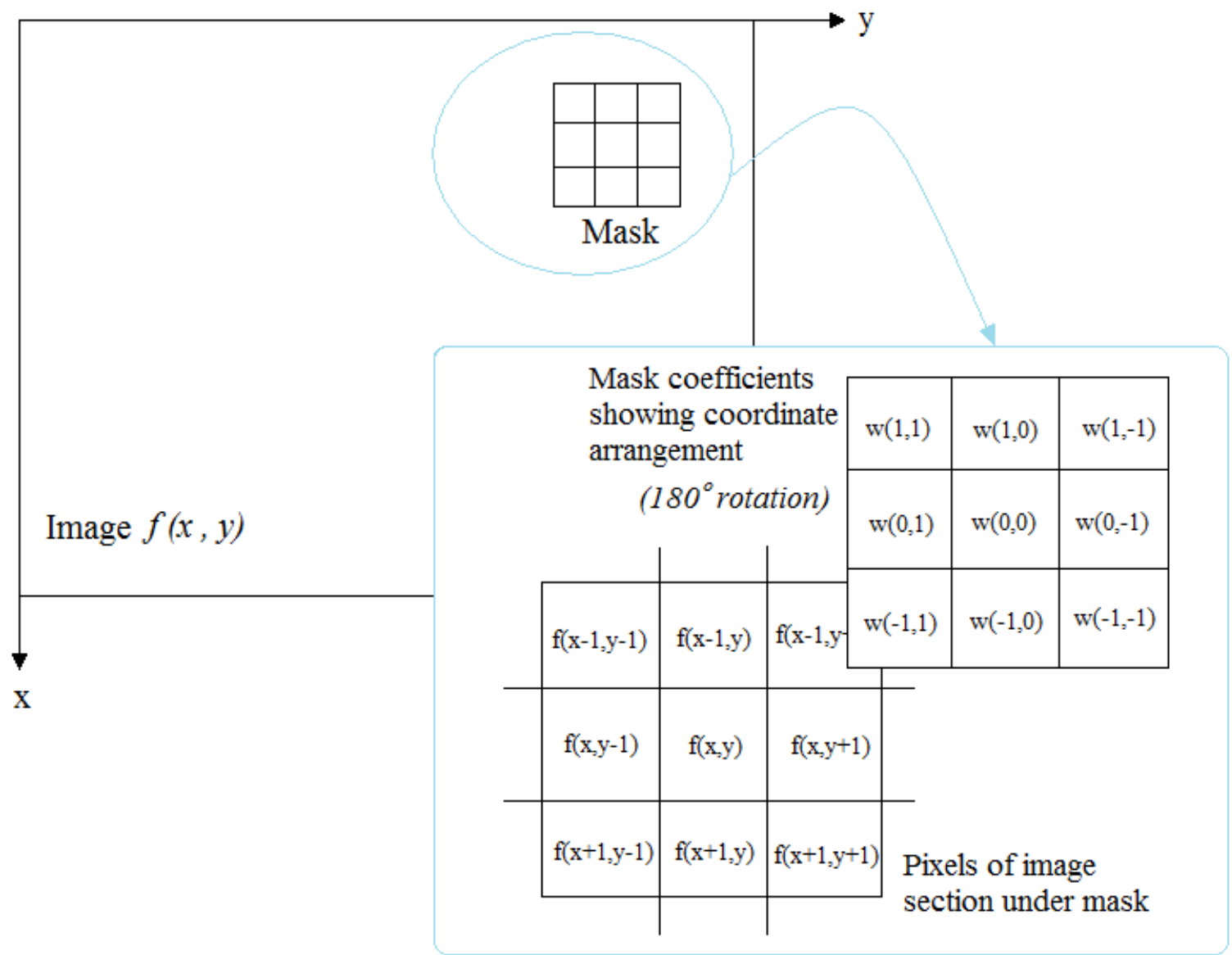

Figure 4.1: Illustration of Linear Spatial Filtering Process (after [91]) 
mask for a variance of 0.25 pixels (standard deviation $=0.50$ pixels) is given by

$$
\operatorname{Gaussian}(\sigma=0.5 \mathrm{pxls})=\left[\begin{array}{lll}
0.0113 & 0.0838 & 0.0113 \\
0.0838 & 0.6193 & 0.0838 \\
0.0113 & 0.0838 & 0.0113
\end{array}\right] .
$$

The effect of motion is realized by applying a uniform mask in the direction of motion, proportional to the relative displacement. For instance, a horizontal displacement of 5 pixels would result in a $1 \times 5$ uniform filter mask. The masks applied for a displacement 5 pixels in the horizontal direction, as well as a mask for a 5 pixel displacement along the diagonal $\left(45^{\circ}\right)$, are given by

$$
\operatorname{Motion}\left(5 \text { pxls, } 0^{\circ}\right)=\left[\begin{array}{ccccc}
0 & 0 & 0 & 0 & 0 \\
0 & 0 & 0 & 0 & 0 \\
0.200 & 0.200 & 0.200 & 0.200 & 0.200 \\
0 & 0 & 0 & 0 & 0 \\
0 & 0 & 0 & 0 & 0
\end{array}\right]
$$

and

$$
\operatorname{Motion}\left(5 \text { pxls, } 45^{\circ}\right)=\left[\begin{array}{ccccc}
0 & 0 & 0 & 0.0501 & 0.0304 \\
0 & 0 & 0.0519 & 0.1771 & 0.0501 \\
0 & 0.0519 & 0.1771 & 0.0519 & 0 \\
0.0501 & 0.1771 & 0.0519 & 0 & 0 \\
0.0304 & 0.0501 & 0 & 0 & 0
\end{array}\right]
$$

respectively.

The overall result is a blurred image. It should be noted that the same result can be obtained by transforming the image to its Fourier domain representation and then multiplying by the transfer function.

The spatial convolution is enabled through the MATLAB imfilter function with the replicate option specified. The replicate option means that for areas where the filter mask is partially out of the image bounds, the image border pixel values are replicated. Other border methods include circular, which assumes the image is periodic, and symmetric, which takes a mirror-reflection of image pixels across the image border. The bounding choice should not matter since the bordering pixels of the photograph tend not to be part of the barcode, but rather the background scene. 
To create defocus blur and motion blur filter masks, the MATLAB function fspecial can be employed with the appropriately specified options. Otherwise, the mask matrix values can be defined directly. For more information, see [92].

\subsection{MATLAB CMOS Camera Response}

Once the image is blurred according to the lens effects, it is re-sampled at the rate of the camera sensor (default is $1600 \times 1200$ pixels). The sampling is done using MATLAB imresize function, using bicubic interpolation. However, the anti-aliasing filter applied by imresize is disabled.

The noise floor and saturation cut-offs are then applied to each of the colour portions of the image. Additional adjustments to the image contrast are applied to model exposure effects (over-exposure, under-exposure). PRNU is also modelled using the Matlab imnoise function, set to speckle. This multiplicative noise is given by $I+n \cdot I$ where $\mathrm{n}$ is a zero-mean uniformly distributed random variable of a set variance.

To model the effects of the Bayer filter, the image is sampled according to a $G B R G$ pattern. Colour filter imperfections are modelled by weighting each colour at a given pixel by a factor dependent on the Bayer colour at that pixel. For instance, a green pixel in the Bayer sensor will consist of a small percentage of the red and blue values at that pixel and a large percentage of the green value at that pixel. Once the Bayer sub-sampling is performed, the MATLAB demosaic function is called to interpolate the colour values, reproducing an RGB image using bilinear interpolation (as discussed in Section 3.4.2).

\subsection{MATLAB Camera Processing and Compression}

Although mobile phones have a limited ability to carry out heavy digital image processing, automatic white balancing (AWB) and contrast stretching are algorithms applied by certain camera phones. White balancing attempts to correct colour imbalances in the photograph caused by external lighting, and many algorithms are available for achieving the desired effects. Automatic white balance is non-adaptive and performs the same algorithm on every photograph. It is less effective than adaptive algorithms, but is currently the only feasible option for camera phones. Gray-World is a common white-balance algorithm which as- 
sumes that a photo should contain an equal level of red, blue, and green colour. It is one of the simplest white-balance algorithms, described by

$$
\begin{aligned}
& \text { avgGray }=(\text { avgRed }+ \text { avgGreen }+ \text { avgBlue }) / 3 \\
& \text { imgOutRed }=\frac{\text { avgGray }}{\text { avgRed }} *(\text { imgInRed }) \\
& i m g O u t G r e e n=\frac{\text { avgGray }}{\text { avgGreen }} *(\text { imgInGreen }) \\
& \text { imgOutBlue }=\frac{\text { avgGray }}{\text { avgBlue }} *(\text { imgInBlue }) .
\end{aligned}
$$

The output images are then scaled by 255 over the maximum pixel value in the image.

If $\mathrm{AWB}$ is not applied, another common technique is to stretch the contrast in each colour channel. In Matlab, the imadjust function can be used with a linear setting to map the input dynamic range to an output dynamic range of 0 to 255 in each colour channel. In general, digital image processing is an optional component of the simulator, as many camera phones do not employ any sophisticated processing.

Once the image processing is complete, a gamma curve with exponent $1 / 2.2$ is applied to each colour channel to model the camera gamma correction, which assumes an sRGB colour space. This process is achieved using MATLAB imadjust function, which applies an exponential gamma curve to a digital image (each pixel value is raised to the gamma exponent).

Once the colour response and gamma-correction functions are applied, the three channels are superimposed to recreate the full RGB image. At this point, the image is written as a JPEG file with a given quality factor, expressed as a percentage.

The MATLAB JPEG compression does conversion from RGB to YUV, where YUV is the $Y C_{B} C_{R}$ specified by ITU-R BT.601-4. The $C_{B}$ and $C_{R}$ channels are down-sampled by a factor of 2 in both directions before the DCT and quantization steps are taken.

Because there are three colour channels, three JPEG normalization matrices are required. For $100 \%$ quality, the JPEG normalization matrices consist of all ones, meaning no quanti- 
zation is applied. The matrices for a quality setting of $50 \%$ are:

$$
Z_{Y}=\left[\begin{array}{cccccccc}
16 & 11 & 10 & 16 & 24 & 40 & 51 & 61 \\
12 & 12 & 14 & 19 & 26 & 58 & 60 & 55 \\
14 & 13 & 16 & 24 & 40 & 57 & 69 & 56 \\
14 & 17 & 22 & 29 & 51 & 87 & 80 & 62 \\
18 & 22 & 37 & 56 & 68 & 109 & 103 & 77 \\
24 & 35 & 55 & 64 & 81 & 104 & 113 & 92 \\
49 & 64 & 78 & 87 & 103 & 121 & 120 & 101 \\
72 & 92 & 95 & 98 & 112 & 100 & 103 & 99
\end{array}\right]
$$

and

$$
Z_{C_{R}}=Z_{C_{B}}=\left[\begin{array}{cccccccc}
17 & 18 & 24 & 47 & 99 & 99 & 99 & 99 \\
18 & 21 & 26 & 66 & 99 & 99 & 99 & 99 \\
24 & 26 & 56 & 99 & 99 & 99 & 99 & 99 \\
47 & 66 & 99 & 99 & 99 & 99 & 99 & 99 \\
99 & 99 & 99 & 99 & 99 & 99 & 99 & 99 \\
99 & 99 & 99 & 99 & 99 & 99 & 99 & 99 \\
99 & 99 & 99 & 99 & 99 & 99 & 99 & 99 \\
99 & 99 & 99 & 99 & 99 & 99 & 99 & 99
\end{array}\right] .
$$

The normalization matrices change with quality settings. For instance, for a setting of $75 \%$, the matrices of Equation 4.3 are divided by 2 and for a setting of $25 \%$, they are multiplied by 2 (where values are rounded up to the nearest integer).

\subsection{MATLAB AWGN Noise}

The final component in the channel model is the AWGN discussed in Section 3.5. The simulator uses the MATLAB imnoise function to add noise to the image. A constant mean and variance is used, with values depending on the level of noise in the system.

The image is first converted from type unit8 to type double. The noise matrix is created using MATLAB randn function, which generates a matrix of normally distributed elements with zero mean and unity variance. The randn matrix is made to have the same dimensions 
as the image and each pixel is then multiplied by the standard deviation and added to the mean of the simulated noise (in this case, zero). For more information, see [93].

The output noisy image is simply the input image plus the noise matrix. Once the image is converted back to type uint8, it is returned.

The AWGN noise takes into account environmental and machine-related imperfections. However, it also considers quantization noise and other image processing non-idealities imposed by the camera before compression. Therefore, it is applied after the camera processing distortion, but before JPEG compression in the overall model. This is not clear from the channel model of Equation 3.1 since the processing and compression are combined into one distortion measure: $J$.

Therefore, in order to clarify any ambiguity with regards to the additive noise, it is added immediately before JPEG compression is applied in the MATLAB simulation.

\subsection{Channel Uncertainty}

From the channel model described in Sections 3 and 4, one can deduce that the various channel parameters lead to an enormous family of potential channels. This is problematic from a communications stand-point. When the channel entails a great degree of uncertainty, effective communication is much more difficult.

Reliable communication under channel uncertainty is a topic of great interest and consequence in the area communications. As a result, the limits of communication over unknown channels have been studied extensively over the past 60 years. In general, the more the encoder and/or decoder know about the family of channels over which transmission is taking place, the easier communication becomes. For a review of some of the significant results for single-user unknown channels, please refer to [94].

In the case of the barcode channel model developed in Chapter 3, the variable parameters clearly lead to an unknown channel. However, if the family of channels can be accurately described, and the limiting channel cases identified, then the communication theory and encoding techniques developed by Shannon and others since may be exploited to communicate effectively.

This section aims to identify the channel parameters and set reasonable limits on the 
degree of variation, thus reducing the scope of the channel to a practical level. Once this is accomplished, the more burdensome combinations of channel parameters (from a communications stand-point) will be identified in order to design for the limiting cases in the family of potential channels.

\subsubsection{Identifying the Variable Parameters}

The variable parameters are given throughout the description of the channel model in Chapter 3 and are listed in Table 4.1.

In order to gain a reasonable degree of control over the simulator, the family of channel models should be reduced to a reasonable level. For instance, although the camera tilt angle can range from -90 to 90 degrees, it is absurd to expect accurate decoding for a photograph taken at an $80^{\circ}$ tilt angle. The photographer has enough control over the phone to orient the camera almost orthographically. Some tilt and pan is anticipated, but the acceptable angles should be limited.

This is true of several of the parameters listed in Table 4.1. In cases where strict limits do not make sense, different levels can be defined, such as in the case the JPEG quality. This will reduce the range of variable parameters to a finite set that sensibly characterizes the family of channels.

\subsubsection{Defining the Range of Variation}

Table 4.2 specifies the range over which the variable channel parameters (see Table 4.1) are defined for the MATLAB channel simulator. The chosen ranges of variation aim to reflect the error expected with camera phone photography.

For the parameters in Table 4.2 that have been assigned an N/A (not applicable) range, some clarification is required.

The focal length of the camera and distance from the barcode to the camera (as well as the physical size of the barcode) are not left variable in the simulator. The only adjustments that can be made are with respect to angles and to the relative size of the barcode (note that there are four valid relative barcode sizes).

The $\mathrm{f} / \#$ of the camera has previously been given a value of 2.8 , typical of cell phone cameras. However, the lens PSF is assumed to be limited by the degree of defocus and 
Variable Parameters

\begin{tabular}{|c|c|}
\hline General & $\begin{array}{l}\text { Resolution of Barcode } \\
\text { Physical Size of barcode } \\
\text { Background Illumination }\end{array}$ \\
\hline Transmitter & Gamma Exponent \\
\hline Warping & $\begin{array}{l}\text { Rotation Angle } \in\left[0^{\circ}, 360^{\circ}\right) \\
\text { Tilt Angle } \in\left(-90^{\circ}, 90^{\circ}\right) \\
\text { Pan Angle } \in\left(-90^{\circ}, 90^{\circ}\right) \\
\text { Size of Barcode vs. } \\
\quad \text { Total Photograph }(\%) \\
\text { Focal Length of Camera } \\
\text { Distance (Barcode to Lens) }\end{array}$ \\
\hline Lens PSF & $\begin{array}{l}\mathrm{f} / \# \text { of Lens } \\
\text { Degree of Defocus } \\
\text { Relative Motion }\end{array}$ \\
\hline CMOS Distortion & $\begin{array}{l}\text { Noise Floor } \\
\text { Well-Capacity } \\
\text { PRNU variance (R,G, and B) } \\
\text { Bayer Filter Sampling } \\
\text { Pixel Cross-Talk } \\
\text { Camera Resolution (pixels) }\end{array}$ \\
\hline Camera Processing & $\begin{array}{l}\text { Gamma Exponent } \\
\text { Other: } \\
\text { Colour-Correction } \\
\text { Contrast Adjustments } \\
\text { etc. }\end{array}$ \\
\hline AWGN & Variance \\
\hline JPEG Compression & Quantization Tables \\
\hline
\end{tabular}

Table 4.1: Variable Parameters in the Overall Channel Model 


\begin{tabular}{|c|c|}
\hline Variable Parameters & Range \\
\hline Resolution of Barcode & any reasonable choice \\
\hline Physical Size of Barcode & $\mathrm{N} / \mathrm{A}$ \\
\hline Transmitter Gamma & any (reasonable) $\gamma$ \\
\hline Background Illumination & \{dark, very bright, well lit\} \\
\hline Rotation Angle & {$\left[0^{\circ}, 360^{\circ}\right)$} \\
\hline Tilt Angle & $\left(-15^{\circ}, 15^{o}\right)$ \\
\hline Pan Angle & $\left(-15^{\circ}, 15^{o}\right)$ \\
\hline $\begin{array}{l}\text { Size of Barcode vs. } \\
\text { Total Photograph (\%) }\end{array}$ & $\{25 \%, 44 \%, 73 \%, 100 \%\}$ \\
\hline Focal Length of Camera & $\mathrm{N} / \mathrm{A}$ \\
\hline Distance (Barcode to Lens) & $\mathrm{N} / \mathrm{A}$ \\
\hline $\mathrm{f} / \#$ of Camera & $\mathrm{N} / \mathrm{A}$ \\
\hline Degree of Defocus & $\sigma^{2} \in(0,4]$ \\
\hline Relative Motion** & $\begin{array}{l}\theta \in[0,360) \\
\quad \# \text { pixels } \in\{0,1, \ldots, 5\}\end{array}$ \\
\hline PRNU variance (R,G, and B) & $(0,0.004]$ \\
\hline Noise Floor & {$[0,30] *$} \\
\hline Well-Capacity & {$[235,255] *$} \\
\hline Bayer Filter Sampling & standard GRBG Bayer pattern \\
\hline Pixel Cross-Talk & $\begin{array}{l}\text { determined experimentally } \\
\text { (net cross-talk after colour correction) }\end{array}$ \\
\hline Camera Resolution (pixels) & $\begin{array}{l}\text { any standard resolution } \\
\text { with } 4: 3 \text { aspect ratio }\end{array}$ \\
\hline Gamma & $\gamma=1 / 2.2$ \\
\hline \multicolumn{2}{|l|}{ Other: } \\
\hline Colour-Correction & GrayWorld White Balance (optional) \\
\hline Contrast Adjustments & Contrast Stretching (optional) \\
\hline AWGN Variance & $\sigma^{2} \in\{0.0001,0.001,0.005\}$ \\
\hline JPEG Quantization Tables & $\begin{array}{c}50-100 \% \text { quality with tables } \\
\text { defined in Section } 4.5\end{array}$ \\
\hline
\end{tabular}

Table 4.2: Range Over which Variable Channel Parameters are Defined 
motion and not by the theoretical cut-off frequency. Therefore, the $\mathrm{f} / \#$ is not a variable in the simulator.

Also, before gamma correction is applied, some camera image processing is modelled by the simulator if desired. White balancing using the Gray-World assumption can be set to be applied, as can individual channel contrast stretching. Complex colour correction is omitted as it is not offered by the majority of camera phones. Also, instead of applying a colourcorrection matrix in the simulator, the colour cross-talk is modelled to be the net leakage after the correction matrix has been applied. The sRGB is assumed to be well approximated by the gamma correction with exponent $1 / 2.2$.

Although the family of channels is now more restricted, it is still very large. The most cumbersome channels in this family should be identified. If reliable communication is achieved for the boundaries of the channel family, it can be expected for the family as a whole.

\subsubsection{The Limiting Cases}

In some unknown channel families, there is one distinct worst-case-scenario. For example, consider the binary symmetric channel (BSC) with cross-over probability $\rho \in S$, where $S$ is some set of values in the range of $[0,1]$. The limiting case for this BSC family is the channel with $\rho$ closest to 0.5 over all elements in $S$. The capacity for this variable BSC is simply the capacity of the of limiting case channel, given by

$$
C=\inf _{\rho \in S}\left(1-h_{b}(\rho)\right)
$$

where $h_{b}$ is the binary entropy function:

$$
h_{b}(x)=-x \log _{2} x-(1-x) \log _{2}(1-x)
$$

for $x \in[0,1]$.

It is interesting that the capacity of the family is the infimum capacity over all channels in the family, simplifying the communication problem to one distinctly defined channel. This result holds for any memoryless family in which the capacity-achieving input probability mass function (pmf) is the same for all channels in the family. [94].

The variable BSC is an example of a compound discrete memoryless channel (DMC). A compound DMC models communication over a memoryless channel whose law is un- 
known but remains fixed throughout a transmission. For the general compound DMC, capacity is achieved with the input distribution that maximizes the infimum mutual information over the entire family. That is, capacity is the maximized infimum mutual information over all channels in the family.

It is clear that the capacity of the compound DMC cannot exceed the capacity of any one channel in the family. However, unlike the compound BSC, the bound may not be tight since different channels in the family may have different capacity-achieving input distributions. The resulting capacity is more complex than for the BSC, but is still well-defined.

The wireless optical camera phone channel is unknown, but appears to exhibit laws that remain fixed throughout a transmission. It may not be a perfectly compound channel, as there are sources of distortion that affect only certain pixels. In addition, the channel does not appear to be perfectly memoryless, as there is inter-symbol interference (ISI) relating to colour mixing, cross-talk, and smear. The capacity calculation of such a channel may not be as simple as for the perfectly compound DMC, but if the family of channels is bounded and well-behaved, a similar approach of maximizing the infimum mutual information may succeed, allowing for reliable communication over the entire family of channels.

In order to distinguish the most cumbersome channels in the family, experimentation was performed using the simulator (after making some adjustments described in the following section). Some of the resultant cases are illustrated in Appendix C. The barcode was ultimately designed to overcome the worst-case channels within the limiting case boundaries in order to deliver a satisfactory degree of robustness.

\subsection{Simulator Adjustments and Histogram Matching}

Clearly, experimentation with a phone, such as the trials described in Section 3.9, is inefficient and unsuitable for larger scale simulations. This is why a channel simulator is essential to the symbology design process. In turn, because the design will rely so heavily on simulation results, the simulator must be a realistic representation of the channel. In order to ensure the reliability of the MATLAB simulator, it was tuned according to the Motorola phone.

It was determined that the phone had a higher degree of colour mixing in the green 
and blur pixels than in the red, making the red channel less error-prone. The phone did not appear to apply a Gray-World AWB algorithm, as done by several other camera phones $[95,96]$. The simulator was adjusted to meet these characteristics. However, because this is not representative of a general camera phone, it is important that different camera models are taken into account when considering channel capacity.

Several barcode photos were taken on an LCD monitor and used to match spatial and frequency domain histograms with barcodes passed through the MATLAB simulator. A certain degree of visual correspondence was also used to adjust the simulator. Achieving an exact match was not attempted because the MOTOROKR is not necessarily representative of the full channel, which assumes any satisfactory camera phone. The purpose was to establish a channel simulator that was within close proximity to a true camera phone channel. Once confidence in the simulator was achieved, the parameters were saved in order to calculate mutual information and further develop the symbology design.

\subsection{Mutual Information Calculations}

In order to design a near-optimal symbology for the camera phone channel, the right processing and encoding techniques must be established. In theory, with a strong mathematical model of the channel, simulation is unnecessary. However, to develop an SDMT approach to barcode encoding, the mathematical model must also describe the channel in the frequency domain. Although the linear components of the camera phone channel are well defined in the frequency domain, some nonlinear components, such as perspective projection, are not. This means that simulation is the only method of studying the effects of different processing techniques and measuring the overall mutual information.

Before an encoding method can be designed, a mathematical model of the channel in the frequency domain, as well as the overall channel capacity, should be estimated. This was accomplished through mutual information calculations, as well as noise analysis, in each of the encoded frequency bins.

Mutual information calculations are central to the design process for the barcode. Therefore, the experimental setup was carefully designed to achieve accurate measurements for mutual information and capacity. In the interest of time, only one transmitting medium was 
evaluated: an LCD. It is expected that if a barcode performs well with an LCD transmission, it will perform well using other surface/paper mediums. The concepts can therefore be extended in future work for paper/surface transmission.

\subsubsection{Experimental Setup}

The MATLAB simulator was used to generate mutual information calculations. Preliminary testing showed that the noise imposed on each frequency bin was different. Therefore, bins were studied independently of each other. To accomplish this, thousands of $400 \times 400$ pixel barcode samples were passed through the MATLAB simulator, acquired (acquisition methods are discussed in Section 5.2.2) and corrected using a perspective correction method (discussed further in Section 5.2.3).

Uniformly distributed values in $[-1,1]$ were loaded into the chosen frequency bins. No processing, other than perspective projection, was applied to the received barcode samples. Mutual information calculations, $I(X ; Y)$, were made for each of the encoded frequency bins based on the input and output distributions, where

$$
I(X ; Y)=\sum_{x} \sum_{y} P_{X, Y}(x, y) \log _{2} \frac{P_{X, Y}(x, y)}{P_{X}(x) P_{Y}(y)}
$$

$P_{X}(x)$ is the probability mass function (pmf) for the input, $P_{Y}(y)$ is the pmf for the output, and $P_{X, Y}(x, y)$ is the joint pmf.

The probability mass functions were estimated based on histograms generated through a large number of samples. To ensure the accuracy and statistical significance of the measurements, an appropriate histogram binning method was required.

\subsubsection{Binning Method}

To use a histogram method to measure mutual information, a sufficient number of bins are required to avoid underestimation. For instance, if the true channel capacity is 8 bits/channel use, any pmf histogram using less than 256 bins will cause the mutual information estimation to be below the true value. There is no theoretical limit to the number of possible $x$ and y values used in Equation 4.5. Therefore, the more bins there are, the more accurate the measurement can become. However, this assumes that there are enough samples to ensure statistical relevance. If there are not enough samples with respect to the number of bins, the 
histograms, particularly the joint histogram, will be too sparse, causing the capacity to be overestimated.

Figure 4.2 illustrates the effect the number of samples has on the calculation of mutual information for a given number of histogram bins. The measurements were made for an AWGN channel with a power constraint of $P=1 / 3$ and a noise variance of $\sigma_{N}^{2}=1 / 4$. The true capacity is given by $C=\frac{1}{2} \log _{2}\left(1+P / \sigma_{N}^{2}\right)=0.6112$ bits/channel use.

The histogram binning method has been studied extensively and has applications in various probabilistic problems [97]. Some study into binning methods has indicated that over 10000 samples per $x$ value (or $x$ bin) is required to achieve accurate mutual information estimates for certain channels [98]. This corresponds to 1,000,000 samples if 100 different input values are desired.

Because a sample for the camera phone channel can only be generated by examining the entire barcode, one million barcodes per trial would be required. At $400 \times 400$ pixels per colour channel, this corresponds to 480 billion transmitted (and 5.8 trillion received) pixel values, which was not feasible for simulation.

In order to make accurate measurements without the required number of samples, a smoothing method was developed to eliminate the unrealistic sparseness in the joint probability mass function. One hundred classes were used (100 bins for the input and output) with 10000 samples. To smooth the joint pmf, two methods were tested: a polynomial fit and a low-pass filter.

The polynomial fit was tested using ten different degrees, and the low-pass filter was tested using a Gaussian distribution with several different kernel sizes and standard deviations. The filter method performed the best, and a kernel size of 11 with standard deviation 2 yielded the closest approximation to the true capacity on known AWGN channels. It was therefore chosen as the smoothing method for simulation, with some adjustment made for very poor channels in which the mutual information is otherwise overestimated. The filter method is compared in Figure 4.3 to the true capacity and statistical calculations with 10,000 and 1,000,000 samples.

The new method generally underestimates mutual information for better channels, which is acceptable for simulation given that the measurements aim to be conservative. 


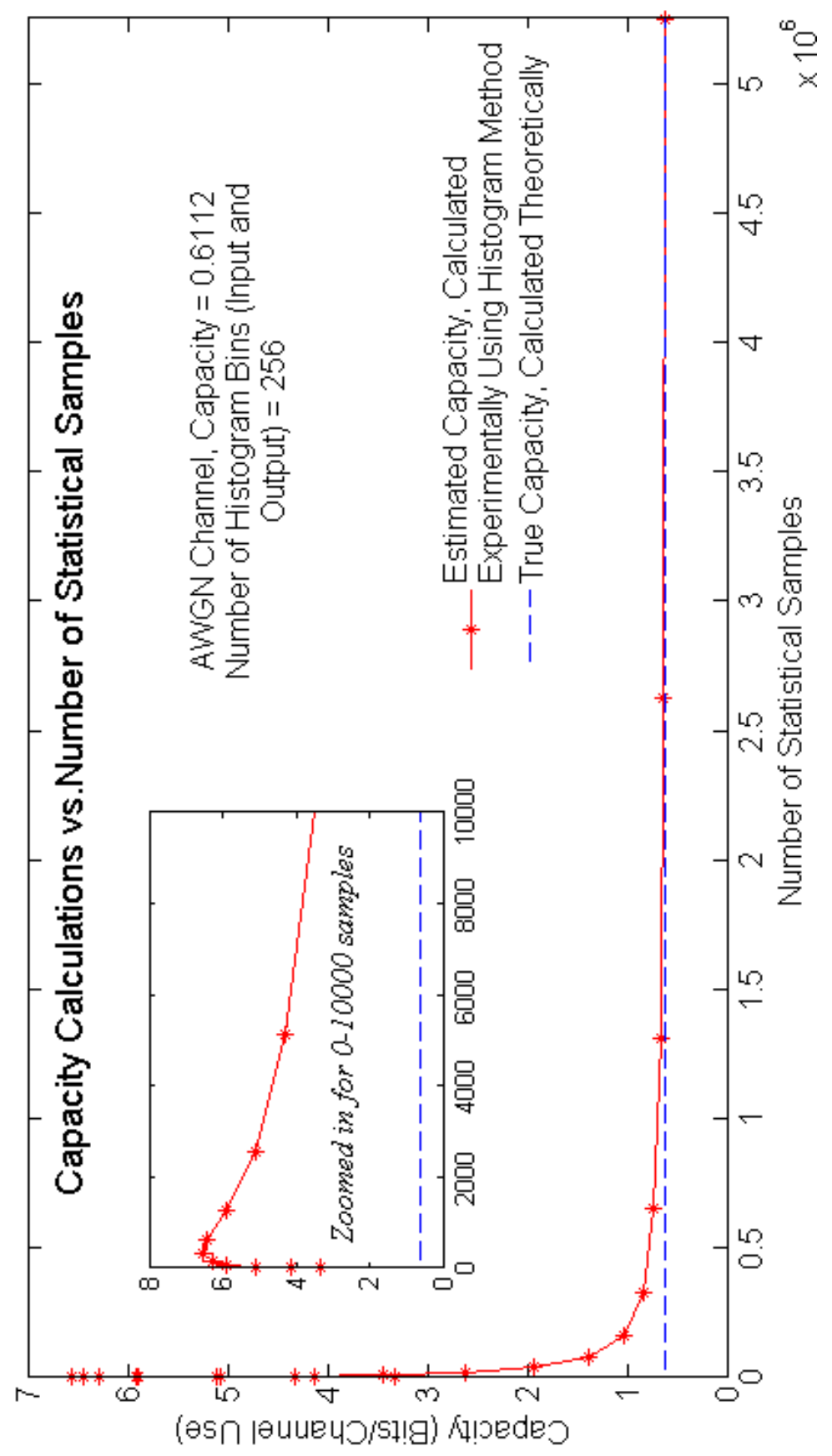

Figure 4.2: Effect the Number of Samples has on Capacity Measurements 


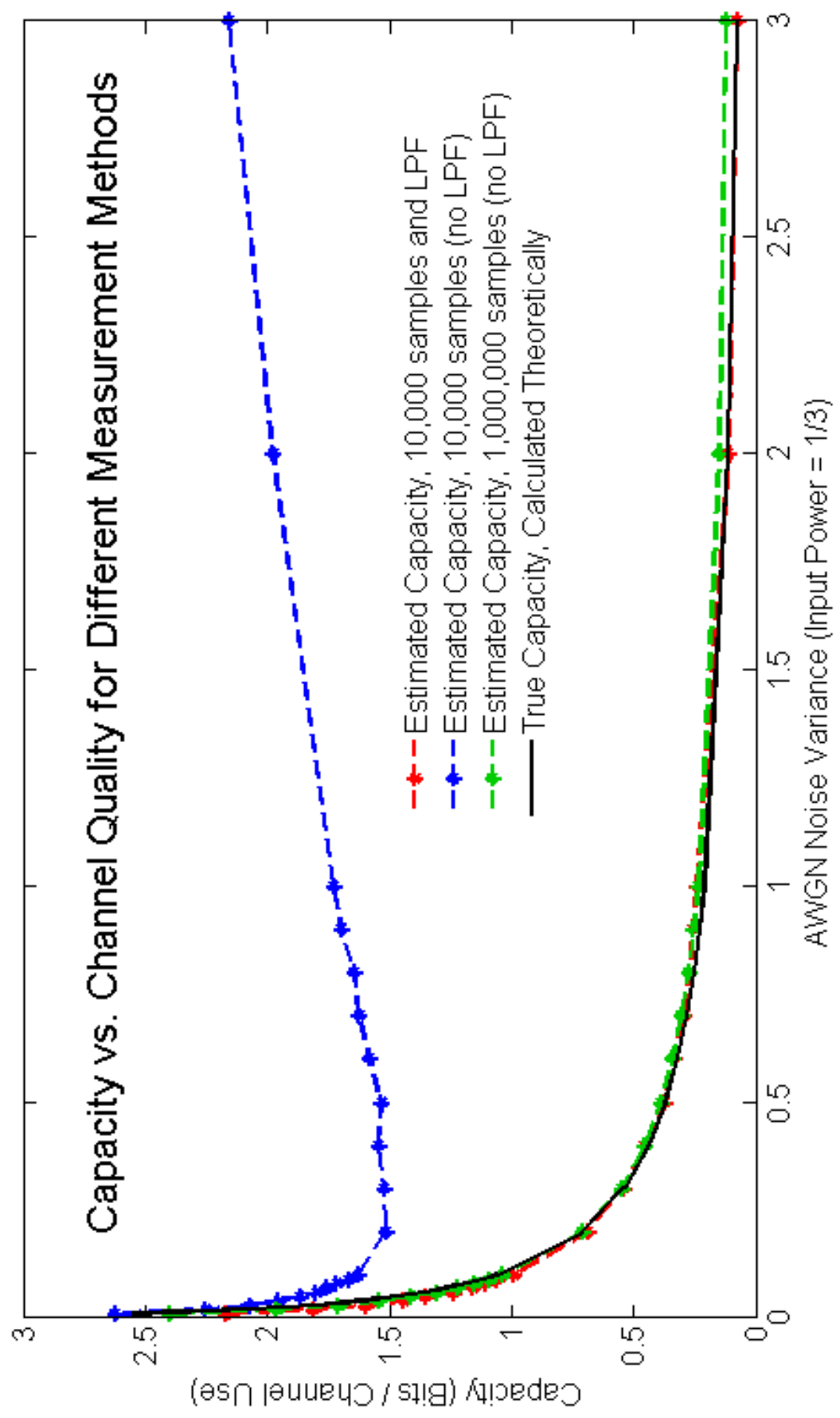

Figure 4.3: Effects of using a low pass filter (LPF) over joint pmf to approximate capacity 


\section{Chapter 5}

\section{Digital Signal and Image Processing}

The basic DCT RGB barcode design was established as a starting point for the symbology design. Other processing techniques are required to aid in acquiring the barcode from the received photograph and to mitigate the effects of distortion. In order to determine what processing steps should be taken at both the transmitter and receiver, different methods were tested and their effect on mutual information was calculated. The processing complexity is not of concern at the transmitter, but is a major consideration at the receiver. Any processing at the receiver must be realistic for a mobile phone processor and a careful costbenefit analysis with respect to the effects on capacity must be made.

To begin the simulations and mutual information comparisons, information was encoded in the DCT domain in the lower frequency bins according to a constant power value in each bin. Zero power was allocated to higher frequency bins. For simulations, barcodes of $400 \times 400$ pixels were used. The number of frequency bins used was left variable (generally the maximum radius from DC for encoded data was between 200 and 350 pixels).

The following sections outline the processing at the transmitter and receiver, and Appendix D contains a simulated photograph before and after the receiver processing steps have taken place.

\subsection{Processing at the Transmitter}

The processing steps at the transmitter include pre-distortion and a noise-shaping algorithm. Figure 5.1 offers a block diagram of the transmitter highlighting the processing steps. The fiducials super-imposed on the barcode prior to transmission are discussed in 


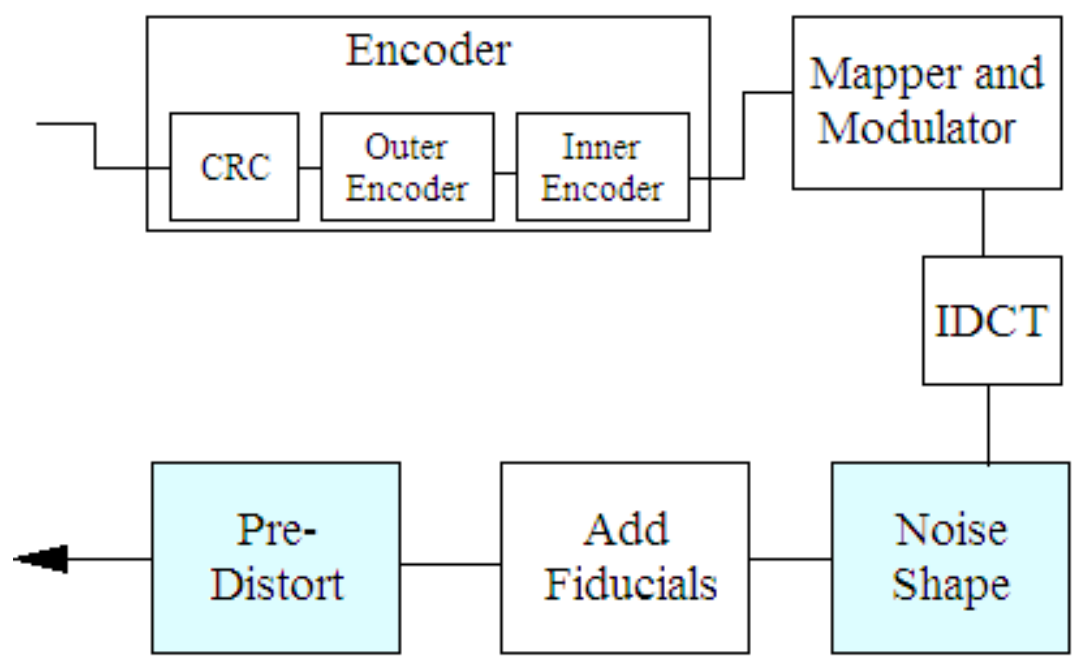

Figure 5.1: Block Diagram of Transmitter Highlighting Signal and Image Processing Steps

Section 5.2.2. The following sections are dedicated to detailing the transmitter processing techniques, as well as a water-filling process that distributes power over the frequency bins according to the noise levels in each bin.

\subsubsection{Pre-Distortion}

Pre-distortion aims to mitigate the effects of transmitter non-linearities by imposing a gamma curve, or contrast adjustment, to the barcode prior to transmission. This is generally only applicable if the transmitting medium is a CRT or LCD display. In order to overcome a computer display gamma distortion with $\gamma=2.2$, a pre-distortion gamma curve with $\gamma=(2.2)^{-1}$ can be applied. As discussed in Section 3.7, an LCD display may be in a state which differs from sRGB due to hardware setting and calibration differences. This makes it difficult to predict what pre-distortion coefficient should be used.

Over many trials, pre-distortion had very little noticeable effect on mutual information even though the simulator gamma was known. The effect is related to the lighting in which the photograph is taken. At times, in darker settings, a gamma distortion unexpectedly improved the results. In general, it is recommended that the barcode be pre-distorted according to the $s R G B$ standard if the transmitting medium is a CRT or LCD. Otherwise, for paper applications, no pre-distortion should take place unless otherwise specified by the printing medium. 


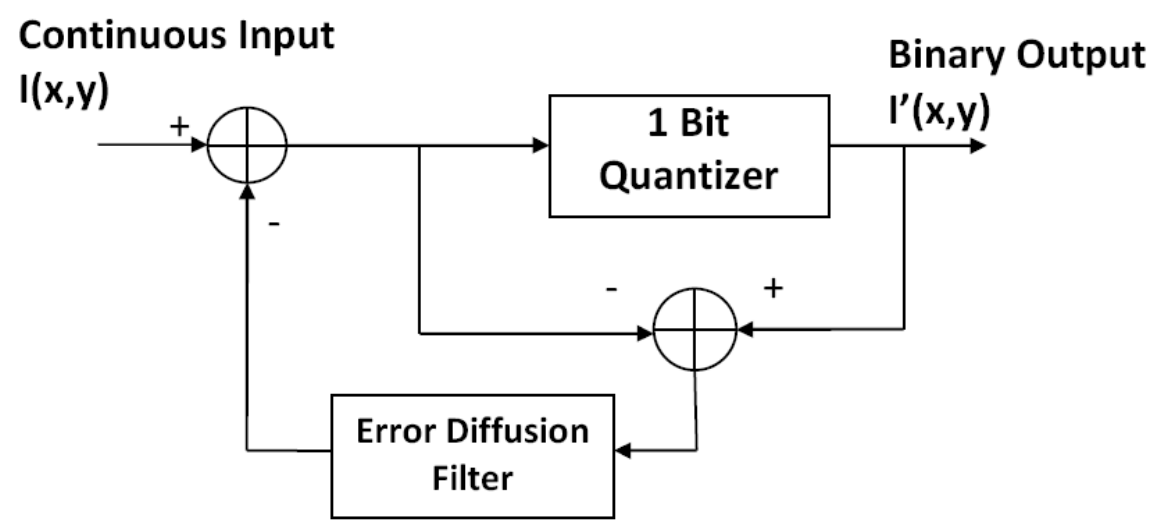

Figure 5.2: Error Diffusion System (after [34])

\subsubsection{Noise Shaping}

Discrete multi-tone modulated signals suffer from a high peak-to-average ratio. In the case of an SDMT barcode, where information is encoded in the frequency domain, clipping in the spatial domain is a significant noise factor. Consider a barcode in which lower frequency bins are encoded with a power value of 1 . Upon taking the inverse Fourier transform, any values outside of $[0,1]$ (where zero corresponds to an intensity value of zero and 1 corresponds to 255) are clipped.

In order to mitigate the effects of the high peak-to-average ratio, the unused high frequency bins can be exploited by using an error-diffusion algorithm or a projection onto convex sets method.

Recall the two-dimensional inverse DCT given by

$$
I(x, y)=\sum_{u=0}^{M-1} \sum_{v=0}^{N-1} F(u, v) \alpha_{M}(u) \alpha_{N}(v) \cos \left(\frac{\pi}{M}\left(x+\frac{1}{2}\right) u\right) \cos \left(\frac{\pi}{N}\left(y+\frac{1}{2}\right) v\right) .
$$

A higher average power level increases the probability of clipping when the inverse DCT is taken to create a real image. Although there is no physical limitation on the amount of power loaded into the bins, the clipping error imposes a considerable constraint.

Noise-shaping is a process that aims to push the clipping noise into unused high frequency bins, where it will be filtered out by the low-pass channel. A noise shaping process is described by Figure 5.2. This technique, called error diffusion, is used often to digitally half-tone images.

Consider a gray-scale image to be printed using only pure black ink. The image must 


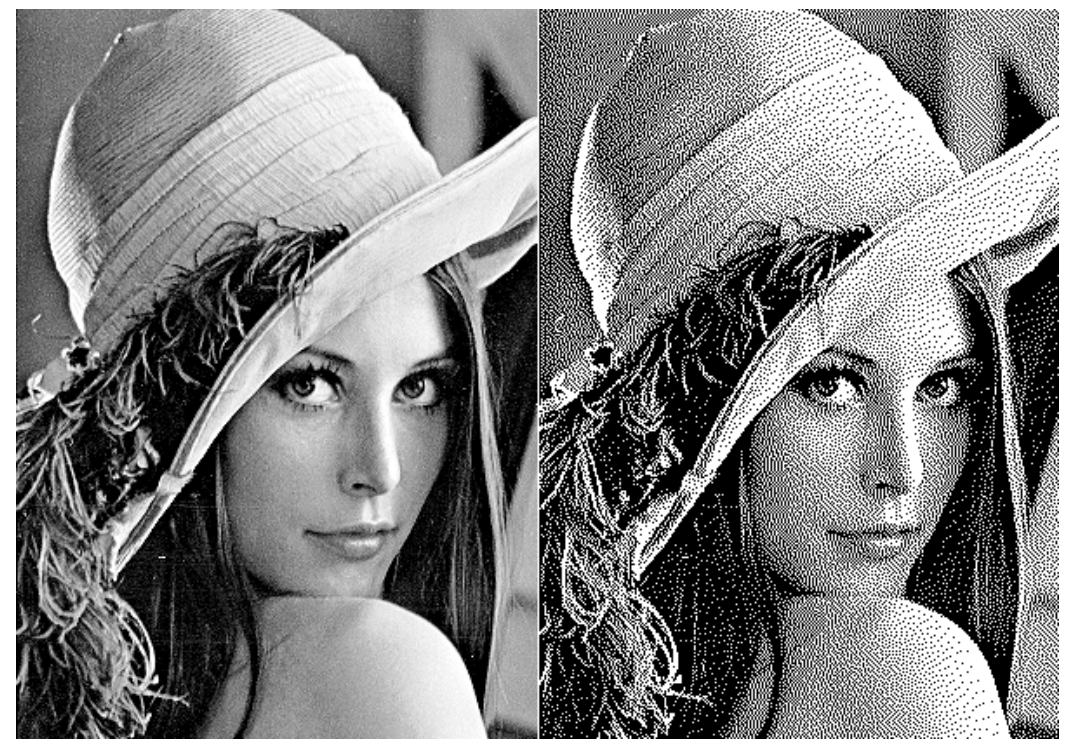

Figure 5.3: Lena: Original (left) and Half-toned (right) using Error Diffusion [99]

be made binary, while still maintaining visual fidelity to the original. The error diffusion system can take the gray-scale image input and digitally half-tone it by filtering the quantization error into high frequency regions. This process reduces the observable error in the image because the eye filters out the high spatial frequencies (see Figure 5.3 in which the right image is a digitally half-toned binary representation of the original image on the left).

The error diffusion filter (see Figure 5.2) is a unity-gain filter with a null at DC. An example of a feedback filter is given by

$$
j(x, y)=\left[\begin{array}{ll}
0 & a \\
a & 1-2 a
\end{array}\right]
$$

and used by Hranilovic and Mohamed [34] in their work on binary half-toned optical intensity channels. Further insight into filter design for the half-tone system is offered by Shoop and Ressler [100].

Readers familiar with sigma-delta modulation, commonly used for analog to digital (AD) conversion, may recognize the error diffusion algorithm to be the extension of sigmadelta modulation to two dimensions.

The error diffusion algorithm can be employed for the DCT barcode design by filtering clipping noise into high frequency regions, where it will be filtered out by the channel.

The noise-shaping method may also be achieved through a projection onto convex sets 


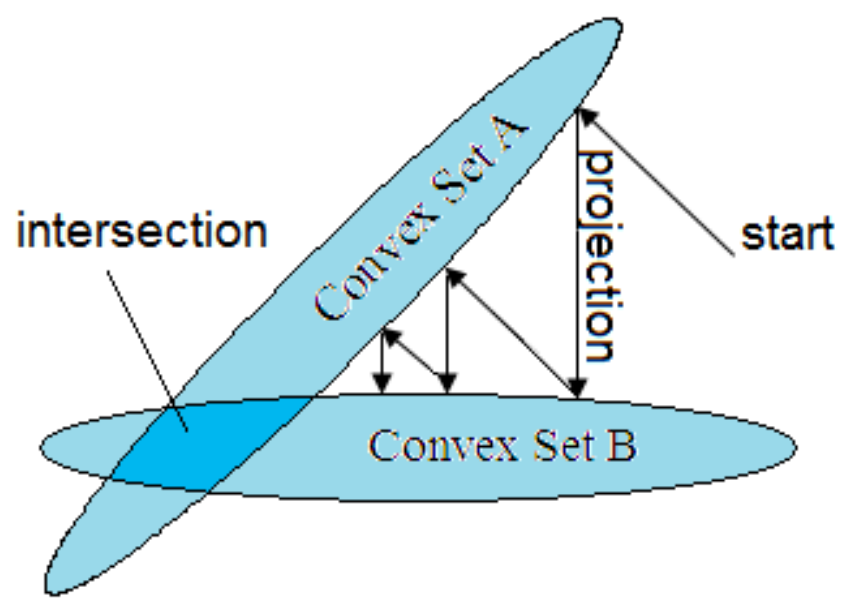

Figure 5.4: Projection onto Convex Sets (after [101])

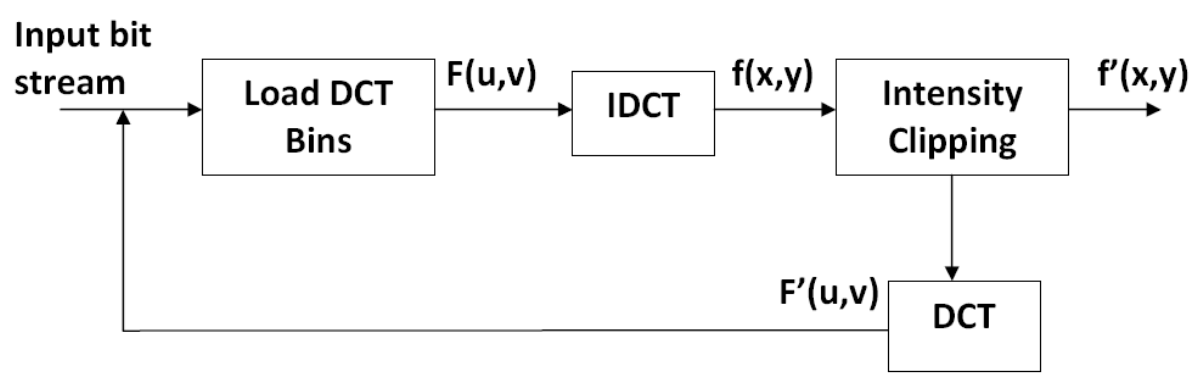

Figure 5.5: Block Diagram of Projection onto Convex Sets Method

(POCS) method. The total power is distributed in the frequency domain, then clipped in the space domain. POCS is a computational approach to finding an element of a feasible region defined by the intersection of a number of convex constraints, starting with an arbitrary unfeasible point [101]. The barcode constraints are 1) the information values loaded into the used DCT bins and 2) the intensity range in the space domain.

By iteratively moving between the DCT domain and the space domain, the area of intersection can be approached. This concept is illustrated by Figure 5.4 and further described by the block diagram in Figure 5.5.

Both the traditional error diffusion method, with various feedback filters, and the POCS method of noise shaping were tested. The POCS method demonstrated a greater reduction in error for a high number of iterations. (Note that this result may change for other filter 
designs and could be studied further.)

Although the computational complexity of the process is high, there is no constraint on complexity at the transmit side. Therefore, the POCs approach with 200 iterations is applied prior to transmission.

\subsubsection{Power Allocation}

The trade-off between power and clipping noise has already been considered, but the distribution of the total power over all frequency bins has yet to be discussed. The power is assigned during modulation, but discussed here because the method is independent of the coding and modulation schemes.

By examining the channel input versus output for individual bins, it was determined that the channel could be modelled as an additive white Gaussian noise (AWGN) channel. Figure 5.6 illustrates the noise observed in different bins and Appendix E outlines the noise measurements in more detail.

It is known that for parallel AWGN channels with a given overall power constraint, the capacity achieving power allocation scheme is achieved through a water-filling process [102]. Essentially, water-filling ensures that the sum of input power and noise power for each channel remains constant. This can be described by

$$
P_{i}=\left(\nu-N_{i}\right)^{+}
$$

such that

$$
\sum_{i=1}^{N}\left(\nu-N_{i}\right)=P_{\text {total }},
$$

where $P_{i}$ denotes the amount of power allocated to channel $i$ (such that $\sum_{i=1}^{N} P_{i}=P_{\text {total }}$ where $N$ is the total number of bins), $N_{i}$ denotes the noise power in channel $i$, and

$$
(x)^{+}=\left\{\begin{array}{l}
x \text { if } x \geq 0 \\
0 \text { if } x<0
\end{array} .\right.
$$

Water-filling allocates more power to the better channels, and less to the noisier channels (a strategy of allocating resources to the most promising areas up to the point of diminishing returns).

For the optical mobile phone channel, the channels are not parallel. There is cross-talk, or power leakage, between channel bins. Therefore, water-filling is unlikely to maximize capacity and the optimal power allocation strategy must be derived as follows. 
Noise Distribution for Red Channel Bin $(1,3)$ over 10,000 Trials

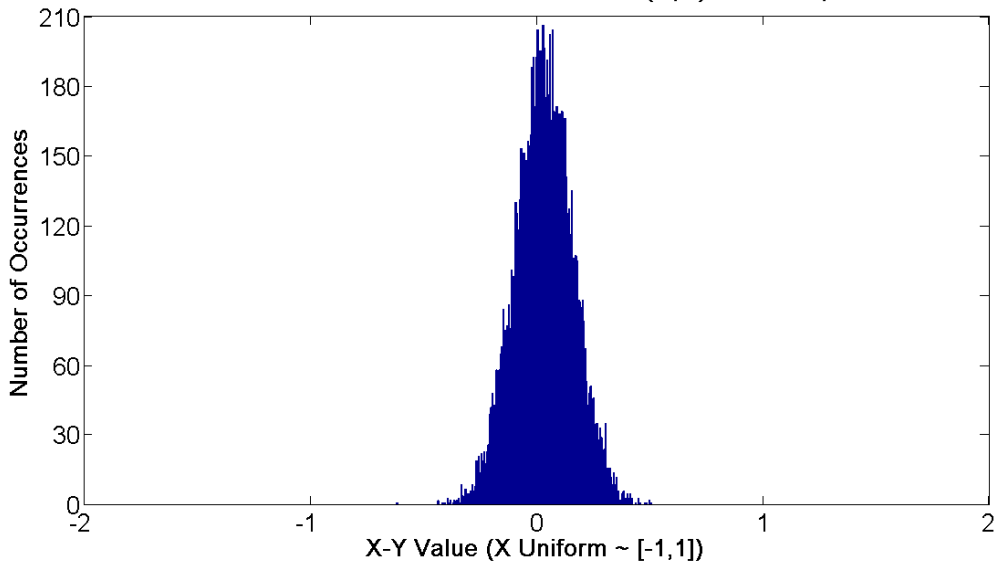

Noise Distribution for Red Channel Bin $(120,120)$ over 10,000 Trials

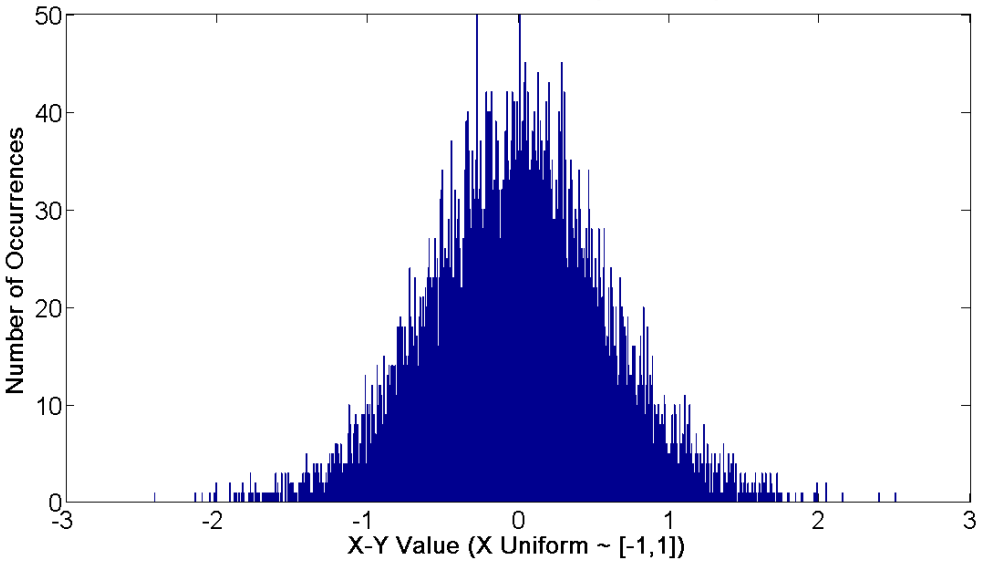

Noise Distribution for Red Channel Bin $(200,200)$ over 10,000 Trials

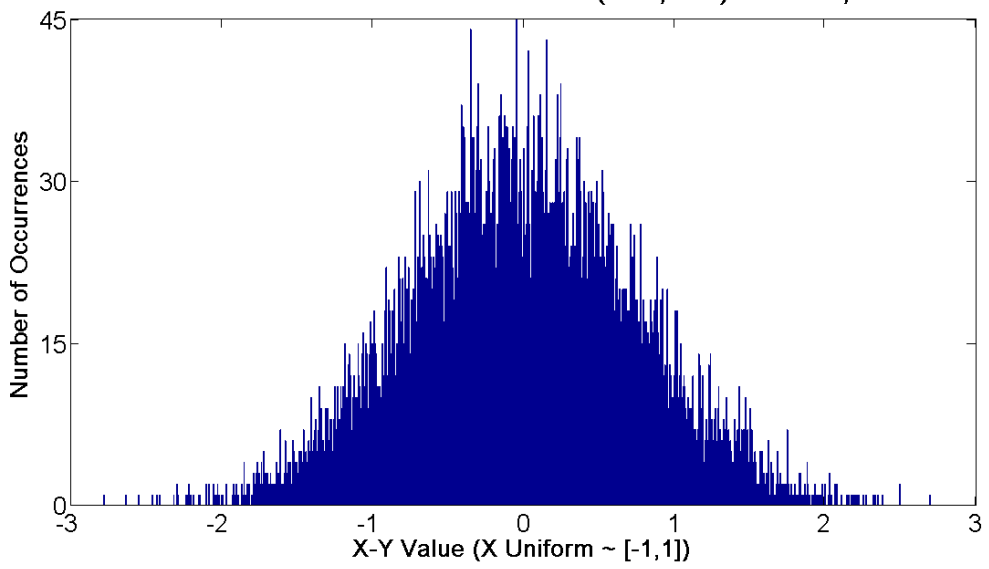

Figure 5.6: Noise Histograms for Different Frequency Bins 
The capacity of the general AWGN channel is related to the input power, $P$ and the noise power, $N$, as

$$
C=\frac{1}{2} \log _{2}\left(1+\frac{P}{N}\right) \text { bits/channel use. }
$$

Therefore, for $N$ parallel AWGN channels, the capacity is given by

$$
C=\sum_{i=1}^{N} \frac{1}{2} \log _{2}\left(1+\frac{P_{i}}{N_{i}}\right) \text { bits/channel use. }
$$

In the case of the optical mobile phone channel, coupling noise (or cross-talk) contributes to the overall noise observed in each channel. Let $\alpha_{i j}$ be the coupling coefficient between channel $i$ and $j$, such that the amount of power that leaks from channel $i$ to channel $j$ is given by $\alpha_{i j} \cdot P_{i}$. Then the total noise power in channel $j$ is given by $N_{j}+\sum_{i \neq j} \alpha_{i j} \cdot P_{i}$. The resultant capacity is given by

$$
C=\sum_{i=1}^{N} \frac{1}{2} \log _{2}\left(1+\frac{P_{i}}{N_{i}+\sum_{j \neq i} \alpha_{j i} \cdot P_{j}}\right) .
$$

Therefore, optimization reduces to maximizing

$$
\max _{P_{1}, \ldots, P_{N}} \prod_{i=1}^{N}\left(1+\frac{P_{i}}{N_{i}+\sum_{j \neq i} \alpha_{j i} \cdot P_{j}}\right),
$$

such that

$$
\sum_{i=1}^{N} P_{i}=P_{\text {total }}
$$

under the power constraint, and the obvious requirement that power be non-negative, $P_{i} \geq$ $0 \forall i$.

To solve the optimization problem, the coupling coefficients must be known. For the optical mobile phone channel, the individual coupling coefficients were observed to be very slight, even though the overall cross-talk is not insignificant.

To measure each of the coupling coefficients, the power in a given bin must be increased such that the effect on subsequent bins can be measured. For the $400 \times 400$ pixel barcodes, this requires a tremendous amount of simulation (roughly 100,000 trials requiring 10000 barcode samples each) and was out of the scope of this thesis. However, optimizing the power allocation remains an open problem which may warrant future study.

Rather than optimizing power mathematically, a practical experimental approach was taken. First, a uniform distribution of power was tested and then a water-filling distribution with the same total power was tested. The water-filling method had a slight advantage in measured capacity ( $\approx 1 \%$ increase in mutual information). However, it had the advantage of significantly increasing the mutual information in lower bins and decreasing the mutual 


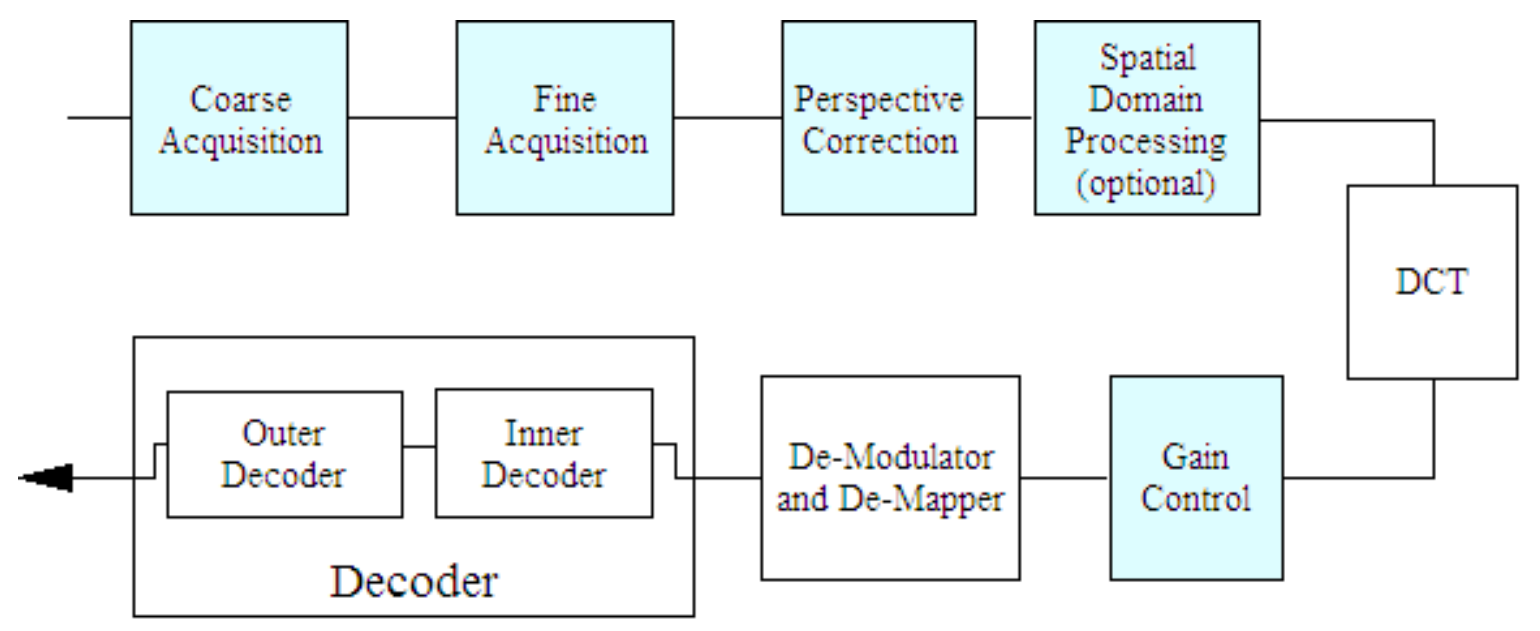

Figure 5.7: Block Diagram of Receiver Including Processing Steps

information of higher bins to the point at which they could be discarded. This has the effect of reducing decoding complexity without a reduction in data density. As a result, the waterfilling approach to power allocation was pursued.

\subsection{Processing at the Receiver}

Once the photograph of the barcode is taken, the receiver must locate the barcode in the photo (acquisition) and apply corrective processing to mitigate the effects of warping, nonideal lighting, and other channel impediments. While processing improves the fidelity of the final barcode before decoding takes place, there are complexity concerns.

Cell phone processors, while improving rapidly, have limited capabilities and can vary significantly. Most cannot perform floating-point computations in hardware, although there are exceptions (the iPhone for instance [103]). There is also variation in the processing speed of mobile phones. For example, there are several mobile phones that use $192 \mathrm{MHz}$ processors, while many new smartphones use a $400 \mathrm{MHz}$ or faster processor [104].

All processing done at the receiver must have a practical implementation for mobile phones, and measures should be taken to reduce complexity as much as possible in order to speed up the decoding process and improve the user experience. Figure 5.7 offers a block diagram of the receiver, highlighting the processing steps. The following sections describe the different image (or signal) processing techniques, as well as efficient methods of performing the two-dimensional DCT. 


\subsubsection{The Fast DCT}

Before discussing the processing steps at the receiver, it is worth examining the 2D-DCT calculation (see Figure 5.7) for complexity considerations. As seen in Equation 3.51, the DCT operation is of order $O\left(N^{2}\right)$, and requires $2(400 \times 400)^{2}=5.12 \times 10^{10}$ multiplications and $2\left((400 \times 400)^{2}-(400 \times 400)\right) \approx 51,200,000,000$ additions for a $400 \times 400$ pixel image. With 3 colour channels, such as in the RGB case, the number of arithmetic computations exceeds 300 billion.

This is a completely unfeasible number of operations at the receiver. Part of the reason the DCT was deemed a reasonable basis choice was because there are fast algorithms that reduce the complexity to $O\left(N \log _{2} N\right)$.

First, consider the receiver structure in Figure 5.7. The DCT is taken prior to decoding, and therefore, only encoded bins are of interest. Let $\alpha N$ denote the maximum encoding radius of the barcode, where $\alpha$ represents a percentage of the barcode dimension $N$. Only the $\alpha N \times \alpha N$ sub-image is of interest at the receiver, so the complexity immediately reduces to $3 \cdot\left(4 \cdot(\alpha N)^{2} \cdot N^{2}\right)$ since the higher frequency bins are not worth calculating. In fact, only $\pi / 4 \%$ of the sub-image contains encoded frequency bins, further reducing the number of bins to compute.

Fast Fourier Transform (FFT) algorithms are widely used as an efficient means for computing the DFT with, reducing complexity from $O\left(N^{2}\right)$ to $O\left(N \log _{2} N\right)$. One general restriction is that the sequence length be a power of 2, though there are algorithms for dealing with sequences of other lengths (the transform can be performed on sets of points corresponding to the prime factors of $\mathrm{N}$ with a slight degradation in efficiency). Further optimizations can be made for base 4 and 8 sequence lengths. See $[105,106,107]$ for more information on FFT algorithms.

Recall that the DCT is the real part of a DFT. Therefore, the FFT of a length-2N real sequence can be computed (requiring half the calculations due to symmetry) to determine the DCT of an N-length sequence in $\frac{1}{2} 2(2 N) \log _{2}(2 N)=2 N\left(\log _{2} N+1\right)$ arithmetic computations (plus some overhead for determining the sequence). This concept is easily extended to two dimensions.

More efficient algorithms that take advantage of highly optimized FFT algorithms exist, further reducing the number of computations. There are also algorithms optimized for the DCT with $O\left(N \log _{2} N\right)$ that do not use an unmodified FFT (though all algorithms are related to the FFT due to the intrinsic relationship between the DCT and DFT). This is an area that has been studied extensively, and several fast techniques for both the DFT and DCT have been developed. The primary purpose of this section is to introduce the concept of the fast 
DCT and assert that one such algorithm must be performed at the receiver, as the regular DCT is impractical. More information about the FFT and fast DCT algorithms can be found at $[105,106,107,108,109,110,111,112]$.

\subsubsection{Acquisition}

There are many barcode acquisition methods and different barcode symbologies employ different techniques. In general, specific border characteristics or fiducials are employed to locate and orient the barcode. For instance, the QR Code (Figure 2.4) uses three large square position detection patterns and the Granite Code (Figure 2.2.6) uses circular bull's-eye fiducials.

For an SDMT approach, perspective correction is required to mitigate the warping effects. Once the barcode is located, a perspective correction process, described in Section 5.2.3, is applied using the four corners as reference points.

The first acquisition method tested was the line scanning method used by Hin Lun Lee [113] and other camera-based barcode acquisition methods [114], which specifies a thin black border around the barcode (approximately eight pixels wide). The method assumes that the barcode fills at least one third of the area in the photograph and is centered. Under the assumption, a border line will intersect with the each of the top and bottom halves of both the left and right borders of the photograph, and each of the left and right halves of both the top and bottom borders of the photograph.

The receiver scans each half of the top and left photograph borders for the intersecting black border line. The angle of the scanning line varies from 0 to 45 degrees in increments of 0.5 degrees. Once the scanning is complete and the four border lines are detected, their intersections are calculated mathematically to determine the location of the four corners of the barcode. Orientation can be indicated by a white spot in the second, third and fourth corners of the black barcode border. For more information on the method, the reader is referred to [113].

The line scanning method was altered to use an eight-pixel-wide blue border with a red square in the first corner. This was to avoid false acquisition for displays on black monitors, where the LCD border could cause black lines through the photograph that interfere with the detection of black barcode border lines. Because detection uses colour, all three colour channels must be considered. To avoid false acquisition, the difference between blue and the sum of green and red pixels is used to detect the border lines. (Otherwise, a white line would correlate as highly as a blue border line.)

The primary downfall of the line scanning method was its complexity, particularly for 
higher resolution photographs since the complexity is of order $O(w h)$. The method requires $3 w h\left(2 a_{h}+2 a_{v}\right)$ computations, where $a_{h}$ and $a_{v}$ represent the number of angles used along the horizontal and vertical directions respectively. Take the example of a $1600 \times 1200$ pixel photograph (that of a 2 Mpixel camera such as the MOTOROKR) and a maximum angle of $45^{0}$ (increments of $0.5^{0}$ ) along each dimension. This corresponds to over 1 billion computations to acquire the barcode at the receiver. This is a significant level of complexity for a camera phone processor, and a serious detriment to the overall barcode design. Furthermore, the assumptions that the barcode is centered and occupying at least $1 / 3$ of the area of the photograph, are restrictive.

A superior acquisition method that both detects and orients the barcode is required with the following characteristics:

- Low Complexity / Fast Acquisition

- Sufficient Accuracy

- Minimal Impact on Data Density

- No Assumptions Regarding Barcode Orientation or Location within the Photograph

- Aesthetically Pleasing (only considered as a secondary objective)

Because both the size and the orientation of the barcode in the photograph are unknown to the receiver, the existent acquisition methods were problematic. For instance, any linear position locators or borders require scanning from various angles, and orientationindependent fiducials, such as those used for Granite Codes, require scanning at various matched filter sizes. To overcome these obstacles, an orientation independent and size independent fiducial was developed.

The resulting acquisition method uses circular coloured fiducials resembling bull's-eyes, as seen in Figure 5.8. The fiducials are specified to be 31 pixels in diameter for a $400 \times 400$ pixel barcode $(7.75 \%$ of the height). The inner red bull's-eye is 11 pixels in diameter $(2.75 \%$ of the height).

The yellow inner ring in the first corner is used to orient the barcode correctly. The circular shape ensures rotational symmetry, and is therefore independent of barcode orientation. The ring thickness ensures size-independence when the receiver correlates with a pattern of two thinner rings, as illustrated in Figure 5.9. This also provides some robustness against warps in which the circular bull's-eyes become elliptical.

A matched filter is used at the receiver to scan over the image and measure correlation with the detection pattern. The dimensions are chosen such that if the barcode consumes 


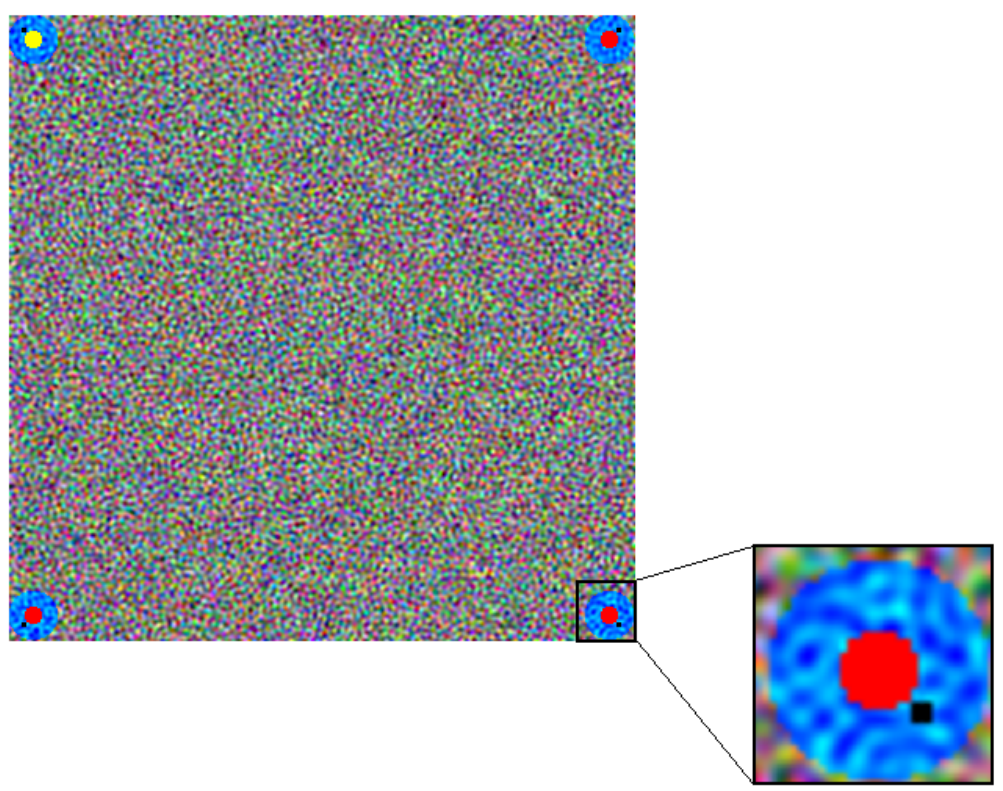

Figure 5.8: Fiducials for Proposed Acquisition Method

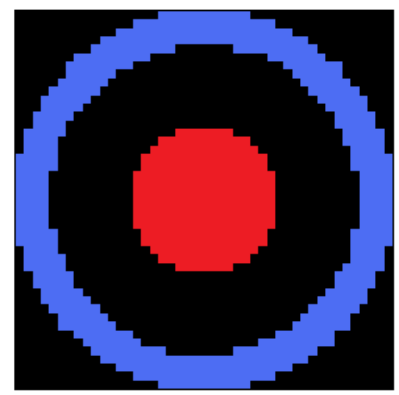

Figure 5.9: Pattern for Receiver Correlation

only $25 \%$ of the total area, acquisition is still successful on the first try. In addition, it is irrelevant to the acquisition method whether or not the barcode is centered. These are two advantages over the previous line scanning method. Note that if the acquisition method is unsuccessful on its first scan of the barcode, it can continue with adjusted thresholds (the limit on the number of attempts depends on decoding time targets).

To reduce the number of computations and increase accuracy, the correlation is performed on the difference between blue and red pixel values. The matched filter pattern contains values of -1 in the inner circle, +1 in the outer ring, and 0 elsewhere. Red and blue are chosen because there is less colour leakage due to their separated wavelengths (as seen in Figure 3.24). This provides for greater accuracy when measuring the difference between the red and blue channels in the matched-filtering process. Because yellow is pure red and 
pure green, the difference between blue and red pixels is the same for yellow as it would be for pure red. This ensures that all four corners, including the first with a yellow inner circle, are detected in the scan.

The computational complexity of the matched-filter acquisition process can be described as

$$
\text { No. Computations }=(\text { Size of Filter }) \times(w \times h) \times 3 \text {, }
$$

where the 3 corresponds to one subtraction (blue-red), plus one multiplication and one addition per matched filter operation. The width of the matched filter is $3.7 \%$ of the minimum dimension of the photograph.

It is unnecessary to apply the matched filter to every pixel in the image. For a $400 \times 400$ barcode displayed on an LCD and photographed by a $1600 \times 1200$ pixel camera, it was found that matched filtering at every 5 pixels along each dimension was more than sufficient. If the fiducial diameters are kept at $7.75 \%$ of the barcode height/width, it is only necessary to match filter across approximately $300 \times 225$ pixels (assuming 4:3 aspect ratio) in the received photograph (even if the barcode size or photograph resolution increases). This reduces Equation 5.7 to

$$
\text { No. Computations }=(\text { Size of Filter }) \times(300 \times 225) \times 3 \text {. }
$$

An important note is that the number of matched-filter operations applied does not scale with camera resolution. Rather, the fixed size of the fiducials with respect to the overall barcode size determines how quickly coarse acquisition can be done. This also allows for a flexible design that can accommodate various applications which may have different datadensity versus acquisition speed requirements.

Assuming the height is the lesser dimension of the received photograph, the size of the filter for the chosen fiducials sizes is

$$
\begin{aligned}
\text { Size of Filter }= & \text { Size of Outer Ring }+ \text { Size of Inner Ring } \\
= & \left(\left(\left(0.037 \cdot h_{R X}\right) / 2\right)^{2} \pi-\left(\left(0.032 \cdot h_{R X}\right) / 2\right)^{2} \pi\right) \\
& \quad+\left(\left(\left(0.015 \cdot h_{R X}\right) / 2\right)^{2} \pi\right) \\
= & 0.00045 \cdot h^{2},
\end{aligned}
$$

resulting in an overall capacity of

$$
91.125 \times\left(h^{2}\right)
$$

Although the complexity remains $O\left(h^{2}\right)$, the fiducial method maintains an advantage over the line scanning method of a factor of 8 fewer computations. (For example, a $1600 \times$ 1200 photograph requires $0.13 \mathrm{~B}$ computations versus $>1 \mathrm{~B}$ for the line-scanning). 
It was hypothesized that because the fiducials were localized in the spatial domain, they would have little effect on the fidelity of the captured barcode in the frequency domain provided that a gray value equal to the received mean intensity (for $\mathrm{R}$ and $\mathrm{B}$ ) replaced the fiducial before further processing and decoding. The actual results proved a $10 \%$ reduction in mutual information (see Appendix E) in comparison to trials in which the fiducials were placed outside of the barcode borders. However, even with the noise imposed by the superimposed fiducials, the method had much greater performance and provided for nearly twice the capacity as the line-scanning method.

A further observation was made in comparing barcodes with super-imposed fiducials to those with fiducials outside the barcode borders. There was a $10 \%$ decrease in capacity when the size of the encoded data was kept constant in the photographs. More realistically, one could re-define the size of the barcode with fiducials outside to be $33 \%$ larger. When these re-defined barcode sizes were kept constant in the photographs, the super-imposed version yielded a higher capacity because the ratio of received pixels to transmitted pixels was greater. Therefore, it is of greater value to super-impose the fiducials onto the barcode in the spatial domain as opposed to wasting valuable real-estate.

It was also observed that including a gray (intensity of 128 for $\mathrm{R}, \mathrm{G}$, and B) border of 1-2 pixels around the barcode improved performance by preventing the photograph background from blurring into the edges of the barcode. A 1 pixel border costs $1 \%$ of the pixel real-estate in a $400 \times 400$ barcode, while a 2 -pixel border costs $2 \%$. It was determined that a 1 pixel border is worth the trade-off, and for particularly blurry applications, a 2 pixel border is recommended.

The method thus far only describes the coarse acquisition method. Once the four corners have been located roughly within the photograph, fine acquisition is performed. As seen in Figure 5.8, there is a black spot, or a fine indicator, in each fiducial to aid in fine acquisition. The fine acquisition imposes a small additional complexity (roughly 20000 more computations), but allows for a significant gain in performance due to a higher accuracy in perspective correction.

The new acquisition method demonstrated a factor of two increase in mutual information when perspective correction is applied (due to greater accuracy) and an $87.5 \%$ decrease in computational complexity, as well as fewer restrictions on the location and size of the barcode within the photograph, in comparison to the line-scanning method. 


\subsubsection{Perspective Correction}

The most significant source of distortion applied to the barcode is the warp due to camera orientation. Equations 3.8 and 3.9 in Section 3.1 express the resultant $x$ and $y$ values in the image plane under a perspective projection.

A perspective correction must be applied to the received photograph, otherwise the encoded information is irretrievable. The perspective correction process is a plane-to-plane homography that maps the received barcode with a warped trapezoidal shape back to its original square (or rectangular) dimensions. The final intensity values in the corrected image are determined by interpolating the received intensity values according to the corrective parameters.

Let $\left(x_{k}, y_{k}\right)$ be coordinates in the received image, and let $\left(X_{k}, Y_{k}\right)$ be the corresponding known coordinates in the transmitted image. The relationship between the two sets of points under the warp is described by

$$
\begin{aligned}
& X_{k}=\frac{h_{11} x_{k}+h_{12} y_{k}+h_{13}}{h_{31} x_{k}+h_{32} y_{k}+h_{33}}, \\
& Y_{k}=\frac{h_{21} x_{k}+h_{22} y_{k}+h_{23}}{h_{31} x_{k}+h_{32} y_{k}+h_{33}} .
\end{aligned}
$$

Therefore, if given the nine unknown values, $h_{11}, h_{12}, h_{13}, h_{21}, h_{22}, h_{23}, h_{31}, h_{23}, h_{33}$, perspective correction can be applied. Written in vector form, $\bar{h}=\left(h_{11}, h_{12}, h_{13}, h_{21}, h_{22}\right.$, $\left.h_{23}, h_{31}, h_{23}, h_{33}\right)^{T}$ and the relationship is described by $A \cdot \bar{h}=0$ where

$$
A=\left[\begin{array}{ccccccccc}
x_{1} & y_{1} & 1 & 0 & 0 & 0 & -x_{1} X_{1} & -y_{1} X_{1} & X_{1} \\
0 & 0 & 0 & x_{1} & y_{1} & 1 & -x_{1} Y_{1} & -y_{1} Y_{1} & Y_{1} \\
x_{2} & y_{2} & 1 & 0 & 0 & 0 & -x_{2} X_{2} & -y_{2} X_{2} & X_{2} \\
0 & 0 & 0 & x_{2} & y_{2} & 1 & -x_{2} Y_{2} & -y_{2} Y_{2} & Y_{2} \\
\vdots & \vdots & \vdots & \vdots & \vdots & \vdots & \vdots & \vdots & \vdots \\
x_{n} & y_{n} & 1 & 0 & 0 & 0 & -x_{n} X_{n} & -y_{n} X_{n} & X_{n} \\
0 & 0 & 0 & x_{n} & y_{n} & 1 & -x_{n} Y_{n} & -y_{n} Y_{n} & Y_{n}
\end{array}\right] .
$$

For $n$ corresponding coordinates, $\left(x_{k}, y_{k}\right)$ and $\left(X_{k}, Y_{k}\right)$, a system of $2 n$ equations in 8 unknowns is obtained where $h_{33}$ is set to one (or some other constant). If $n=4$, then an exact solution is obtained. Otherwise, if $n>4$, the matrix is over-determined, and $\bar{h}$ is estimated by a suitable minimization scheme [115].

The accuracy of the estimated $\bar{h}$ values depends both on the errors in the position of the points used for its computation, as well as the estimation method used. There are three standard methods for estimating $\bar{h}$ [115]: 
1. Non-homogeneous linear solution

2. Homogeneous solution

\section{Non-linear geometric solution}

The non-homogeneous linear solution method was chosen due to its low complexity. It is accomplished by finding the four corners of the received barcode via the fiducial acquisition method, $\left(x_{1}, y_{1}\right),\left(x_{2}, y_{2}\right),\left(x_{3}, y_{3}\right),\left(x_{4}, y_{4}\right)$, which correspond to the true barcode corner coordinates: $\left(X_{1}, Y_{1}\right),\left(X_{2}, Y_{2}\right),\left(X_{3}, Y_{3}\right),\left(X_{4}, Y_{4}\right)$. Then $h_{33}$ is set equal to one to solve for

$$
\bar{h}=A^{-1} \cdot \bar{R}
$$

where

$$
\begin{gathered}
\bar{h}=\left(h_{11}, h_{12}, h_{13}, h_{21}, h_{22}, h_{23}, h_{31}, h_{23}\right)^{T}, \\
\bar{R}=\left(X_{1}, X_{2}, X_{3}, X_{4}, Y_{1}, Y_{2}, Y_{3}, Y_{4}\right)^{T},
\end{gathered}
$$

and

$$
A=\left[\begin{array}{cccccccc}
x_{1} & y_{1} & 1 & 0 & 0 & 0 & -x_{1} X_{1} & -y_{1} X_{1} \\
0 & 0 & 0 & x_{1} & y_{1} & 1 & -x_{1} Y_{1} & -y_{1} Y_{1} \\
x_{2} & y_{2} & 1 & 0 & 0 & 0 & -x_{2} X_{2} & -y_{2} X_{2} \\
0 & 0 & 0 & x_{2} & y_{2} & 1 & -x_{2} Y_{2} & -y_{2} Y_{2} \\
\vdots & \vdots & \vdots & \vdots & \vdots & \vdots & \vdots & \vdots \\
x_{n} & y_{n} & 1 & 0 & 0 & 0 & -x_{n} X_{n} & -y X_{n} \\
0 & 0 & 0 & x_{n} & y_{n} & 1 & -x_{n} Y_{n} & -y_{n} Y_{n}
\end{array}\right]^{-1}
$$

This method has been implemented on the Motorola phone [113], and a simplification for reduced complexity is offered by [114].

To reduce the amount of error, fine acquisition could locate more than just four points. In addition, the least squares problem could be solved using singular value decomposition (SVD) - the homogeneous solution method. (A non-linear geometric solution, which aims to minimize the Euclidean distance between measured and mapped points, is unfeasible on a phone because there is no closed form.)

With $n$ points identified in the received image, matrix $A$ of Equation 5.12 becomes a $2 n \times 9$ matrix (where $2 n>9$ ). The homogeneous solution method solves this over-determined least squares problem and is obtained using singular value decomposition (SVD). From linear algebra, the solution to the homogeneous system, $\bar{h}=\left(h_{11}, h_{12}, h_{13}, h_{21}, h_{22}, h_{23}, h_{31}\right.$, $\left.h_{23}, h_{33}\right)^{T}$, is the eigenvector corresponding to the smallest eigenvalue of matrix $A^{T} A$, which can be obtained directly from the SVD of A $[115,116,117]$. 
In future versions of the barcode, particularly given greater processing capabilities on mobile phones, more than four points should be sought in acquisition, and perspective correction should use an over-determined system of equations, solved through the homogeneous solution method. The fiducial method of Section 5.2.2 can still be used, with a small adjustment of adding more small fine indicators (see the black spots in Figure 5.8) and updating the receiver to search for all the fine indicators.

Once the $\bar{h}$ values have been solved for, the perspective correction process is carried out as follows using a bilinear interpolation method:

for every $(i, j)$ spatial coordinate in the transmitted barcode:

1) compute Matrix $B$

$$
B=\left[\begin{array}{cc}
\left(h_{31} \cdot i\right)-h_{11} & \left(h_{32} \cdot i\right)-h_{12} \\
\left(h_{31} \cdot j\right)-h_{21} & \left(h_{32} \cdot j\right)-h_{22}
\end{array}\right]
$$

2) compute Matrix $C$

$$
C=\left[\begin{array}{l}
h_{13}-\left(h_{33} \cdot i\right) \\
h_{23}-\left(h_{33} \cdot j\right)
\end{array}\right]
$$

3) if $\operatorname{det}(B) \neq 0$, find $p_{1}, q_{1}, p_{2}$, and $q_{2}$ where

$$
\begin{aligned}
& p_{1}=\text { floor }(x) \\
& q_{1}=\operatorname{floor}(y) \\
& p_{2}=\operatorname{ceil}(x) \\
& q_{2}=\operatorname{ceil}(y) \\
& \text { where }(x, y)^{T}=\left(B^{-1}\right) \cdot C
\end{aligned}
$$

floor $(x)$ refers to the nearest integer not greater than $x$, $\operatorname{ceil}(x)$ refers to the nearest integer not less than $x$

4) based on the four nearest coordinates in the received image: $\left(p_{1}, q_{1}\right),\left(p_{1}, q_{2}\right),\left(p_{2}, q_{1}\right)$, and $\left(p_{2}, q_{2}\right)$, bilinear interpolation is performed to find the resultant intensity value at $(i, j)$ in the corrected image. Bilinear interpolation is described by 


$$
\begin{aligned}
I_{\text {corrected }}(i, j)= & (1-a)(1-b) I_{R X}\left(p_{1}, q_{1}\right)+(a)(b) I_{R X}\left(p_{2}, q_{2}\right) \\
& +(1-a)(b) I_{R X}\left(p_{1}, q_{2}\right)+(a)(1-b) I_{R X}\left(p_{2}, q_{1}\right),
\end{aligned}
$$

where $a$ and $b$ are equal to the distance from $x$ to $p_{1}$ and

y to $q_{1}$ respectively.

A higher degree of accuracy can be obtained by using a higher order interpolation method. However, for cell phone processors, the gains in accuracy do not outweigh the increase in complexity. Even when using a bilinear method, the complexity of the perspective correction is high (roughly 55 add/multiplies per transmitted pixel). However, it is a necessary processing step, crucial to a successful decode, and therefore the computations must be afforded.

\subsubsection{Gamma Correction}

As previously discussed, cameras apply a gamma curve to the raw image before the JPEG compression is applied. If this is the final non-linearity applied by the camera processing unit (as it is in the channel model), it is possible to undo it at the receiver by applying an inverse gamma curve. It is estimated that the camera gamma curve uses a coefficient of $1 / 2$.2. This can be confirmed through a training, or calibration, procedure done on individual mobile phone before the user wishes to begin photographing barcodes.

To undo the effects of the camera gamma correction with coefficient $1 / 2.2$, a new gamma curve with coefficient 2.2 is applied to the received image. This can be accomplished by using a look-up table (LUT) and correcting each of the three colour channels individually (using the same LUT). There are 256 possible gray-scale colours, so an LUT of size 256 is required, corresponding to a gamma coefficient of 2.2. If the calibration process determines that the gamma coefficient of the phone is not $\approx 1 / 2.2$, this value is overwritten in software and a new LUT is generated using set-up software.

The complexity of the gamma-correction is one look-up operation per pixel per colour channel: $3 \times h \times w$. Therefore, for a $400 \times 400$ barcode, there are 480,000 read/writes. Gamma correction was originally hypothesized to be an important processing step, but tests suggest that gamma correction has a small effect on reducing error. Therefore, in the interest of achieving fast decoding, the gamma correction step may be skipped completely. 


\subsubsection{Contrast Stretch}

Contrast stretching was another corrective procedure that was tested. By increasing the dynamic range of the received photograph, it was hypothesized that the effects of dim-lighting and other channel impairments could be overcome. However, while contrast stretching did improve the fidelity of some received photographs, the overall effect over many photographs was negative. Given the complexity of the procedure, it was determined that contrast stretching is not a worthwhile processing step at the receiver.

\subsubsection{Colour Correction}

As previously discussed, camera phones apply a colour correction matrix to the raw image to overcome the effects of colour leakage in the Bayer colour filters. It was thought that through a calibration procedure, the residual colour-mixing in the final photographs could be estimated and corrected for at the receiver using a second colour-correction matrix.

Although there is colour-mixing in the final photograph, accurate estimations were difficult to make because of the non-linearities imposed after the CMOS filter (such as the gamma curve and JPEG compression). Therefore, the colour-correction procedure had a limited effect on performance despite a fairly high complexity. It is therefore not applied at the receiver.

\subsubsection{Gain Adjustments}

Gain adjustments in both the spatial domain and the DCT domain can be made at the receiver.

In the spatial domain, the mean pixel value for each of the three colour channels is adjusted to 128. To overcome shadowing effects, this gain adjustment can be done locally using more advanced algorithms. There will inevitably be a trade-off with complexity. For the lighting arrangements in the simulations, the spatial gain adjustments had little effect on the mutual information. However, if shadowing later becomes of greater concern, spatial domain gain adjustments will warrant further study.

If Gray-World AWB (see Section 4.5) is anticipated (or determined through a calibration process done on the mobile phone), spatial domain gain control is required. While GrayWorld AWB has little to no effect when it is applied to the barcode, it can have a strong detrimental impact when the barcode is contained in a photograph with a dominant background colour. The AWB may render the barcode undecodable in such a case, but a global gain adjustment over the acquired barcode easily mitigates these effects. As a precaution, 
unless calibration is carried out on each phone with training images/barcodes, a global spatial domain gain adjustment is a recommended step at the receiver.

Frequency gain adjustments are more crucial to accurate decoding. The gain must be adjusted regionally to overcome attenuation that varies according to the distance from DC. The adjustments are made regionally where the regions are assigned according to the bins' distance from DC. Roughly 2000 bins are contained within a region.

Before gain adjustment is commenced, the bins are normalized in amplitude. Each bin is multiplied by $P(i, j)^{-1 / 2}$, where $P(i, j)$ is the power allocated to bin $(i, j)$ according to the water-filling process discussed in Section 5.1.3.

The first gain control method tested estimates the gain over each region and multiplies each bin in the region by the absolute value of the average transmitted amplitude divided by the estimated gain. The gain estimation over a region is calculated to be the unbiased estimator of the sample mean, which is the expected value of the gain over all samples in the region. A variation of the method was designed to account for small variations from the average correlated to a bin's radius from DC. The gain adjustment is taken to be the estimated value for the radius splitting the region into two equal areas. For each radial increment in the DC direction, the gain is increased by $0.65 \%$, and vice versa for increments away from DC.

A second approach for frequency gain control was tested using pilot tones in each region to assess the attenuation factor in the region. Because the attenuation is a factor of more than the lens PSF, and can vary significantly even along a given radius, the pilot tone approach performed less well that the previous method. The pilot tone approach had the further disadvantage of requiring bins to be left un-encoded.

While frequency gain adjustments do not demonstrate an effect on mutual information, they are essential for accurate decoding and log-likelihood measurements. Therefore, frequency gain control (the first approach) is taken at the receiver after all of the spatial domain processing steps are complete and the DCT has been taken.

\subsubsection{Calibration and Other Image Processing}

At this point, no other processing is recommended to be applied at the receiver. However, if a training process in conducted (by the phone user) in which a calibration barcode/image is assessed by the phone's software, other valuable processing may be determined worthwhile. For instance, if the phone has a very poor colour response, a red, green and blue LUT could be generated to correct all future photographs. There are clearly trade-offs with complexity which the software developer must take into account. 


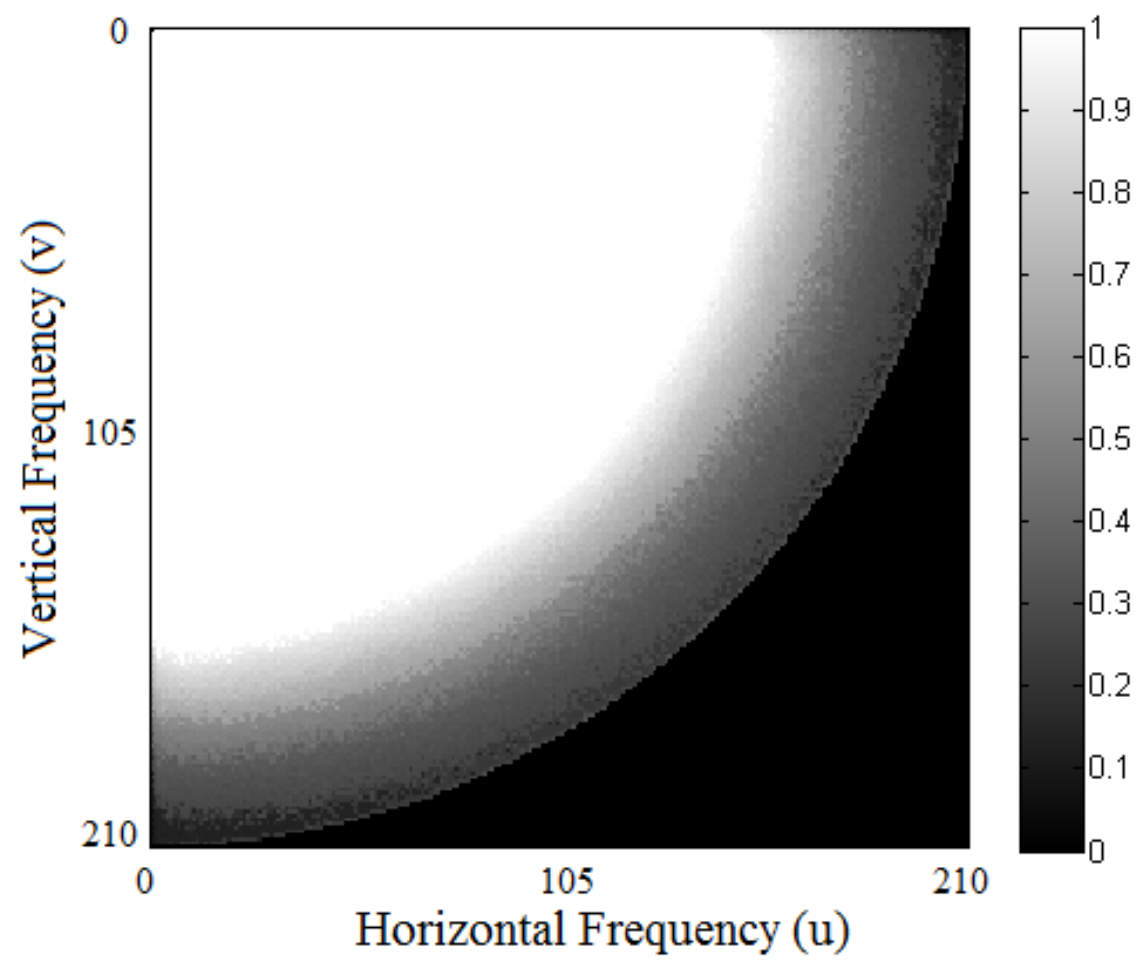

Figure 5.10: Image Representation of Estimated Capacity in DCT Domain (Scale capped at 1 bit/symbol), $400 \times 400$ Barcode, Maximum Encoding Radius = 210, Fiducial Diameter $=31$ pixels, 2-pixel-wide Gray Border

\subsection{Estimated Capacity}

Once all the processing techniques were established, the mutual information of the resultant barcode was measured to attain an estimate on capacity. The simulator settings for the capacity measurement are outlined in Appendix C. The resultant capacity, or the sum of the capacities for every frequency bin in all three colour channels, was estimated to be 58,509 bits (just over 7.3 KB). Figure 5.10 illustrates the capacity in the DCT domain, which varies from bin to bin. It is clear from the figure that the capacity of a given bin is a function of its radial distance from $\mathrm{DC}$.

Appendix E constrains the full range image representations of the capacity estimates over the DCT domain, as well as the capacity when the fiducials are placed outside of the barcode borders (i.e. not superimposed over the data region).

The capacity also varies with colour. The colour breakdown was: 21,603 bits for red, 22,188 bits for green, and 14,718 bits for blue. It was expected that green would have a high capacity because of the Bayer filter and the JPEG compression standard (the chrominance 
red and blue are down-sampled, but the luminance is not). Furthermore, the superimposed fiducials are detrimental to the red and blue channels, while they have little effect on the green channel.

While it was expected that red would have a higher capacity than blue by a small factor (red generally has a higher sensitivity response in Bayer filters [76, 77, 78]), red outperformed blue by a factor of $50 \%$. This was partly because the colour mixing into red was less substantial. Although this is the case for the MOTOROKR, it may not be the case for other phones with different Bayer filters and different colour-correction matrices. Therefore, it can be assumed that red and blue will both have the roughly the same capacity: 14,718 bits. This reduces the overall capacity estimate to a more conservative 51,623 bits (6.5 KB).

A reasonable target for the final barcode is to achieve at least $80 \%$ of capacity, thus attaining a density of $5.2 \mathrm{~KB}$ in the $400 \times 400$ sized barcode. 


\section{Chapter 6}

\section{Modulation and Coding}

Modulation and coding are the final steps taken in the barcode design. This section is dedicated to detailing the encoder and decoder, as well as the modulator/mapper and demodulator/de-mapper, in the transmitter and receiver respectively (Figures 6.1 and 6.2). Modulation refers to the method by which the DCT basis functions are varied in order to convey the message encoded in the barcode. In the DCT domain, pulse-amplitude modulation (PAM) is the natural choice and the methods used are discussed further in Section 6.1.

Channel coding is used to protect the encoded data in the barcode in the presence of noise. Without channel coding, achieving a near-capacity rate is not possible. However, there are very powerful codes, such as Turbo and LDPC codes, that do allow near-capacity data transmissions.

It was seen that the estimated capacity of the barcode with a maximum radius of 210 is $6.5 \mathrm{~KB}$. This provides an upper bound target for the data density of the barcode with a successful coding scheme.

As seen in Figure 5.10 and Figure E.1 (Appendix E), the capacity per DCT bin varies throughout the barcode, and decreases as a function of radial distance from DC. It is clear that one coding method cannot accommodate every region of the barcode since the error rate of a code is dependent on SNR. A largely variable SNR would require a very low-rate code to be employed over the barcode, and the high-capacity bins near DC would essentially be wasted.

To overcome this inherent intricacy, an inner code must be used to encode different radial frequency regions with different rate code. Then a powerful outer code can be employed to achieve performance targets. This structure is reflected in the block diagrams of Figure 6.1 and 6.2. A cyclic-redundancy check (CRC) is added to the input data stream at the transmitter (Figure 6.1) in order to provide error-detection capability at the receiver and 


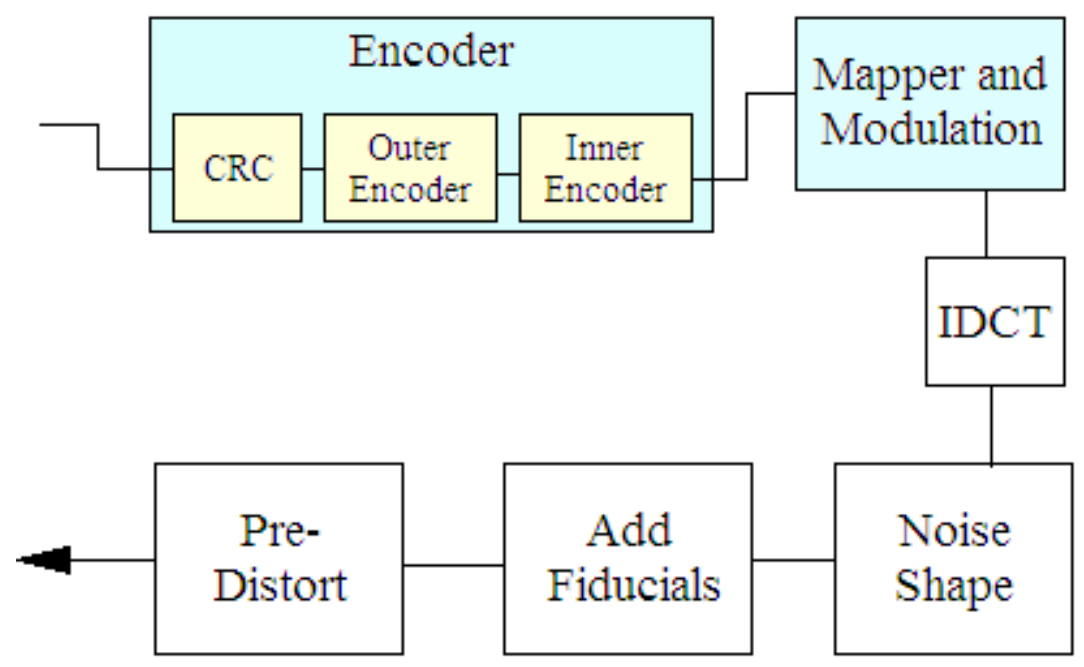

Figure 6.1: Transmitter Block Diagram Highlighting the Encoder and Modulator/Mapper

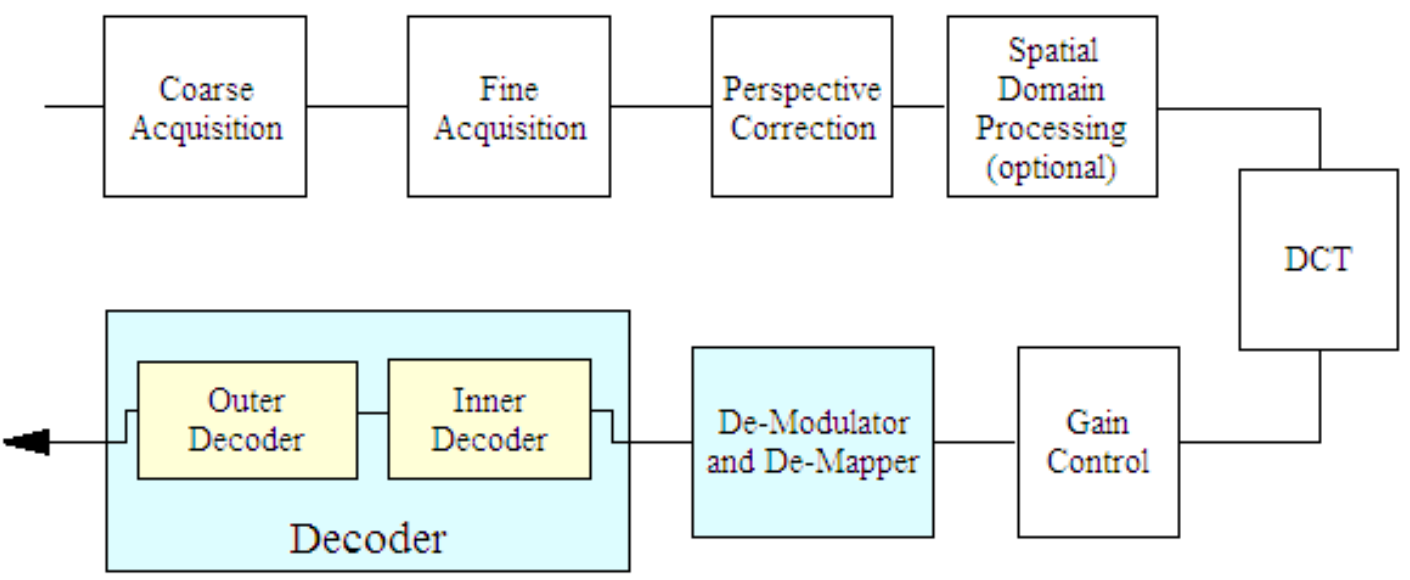

Figure 6.2: Receiver Block Diagram Highlighting the Decoder and Demodulator/DeMapper 


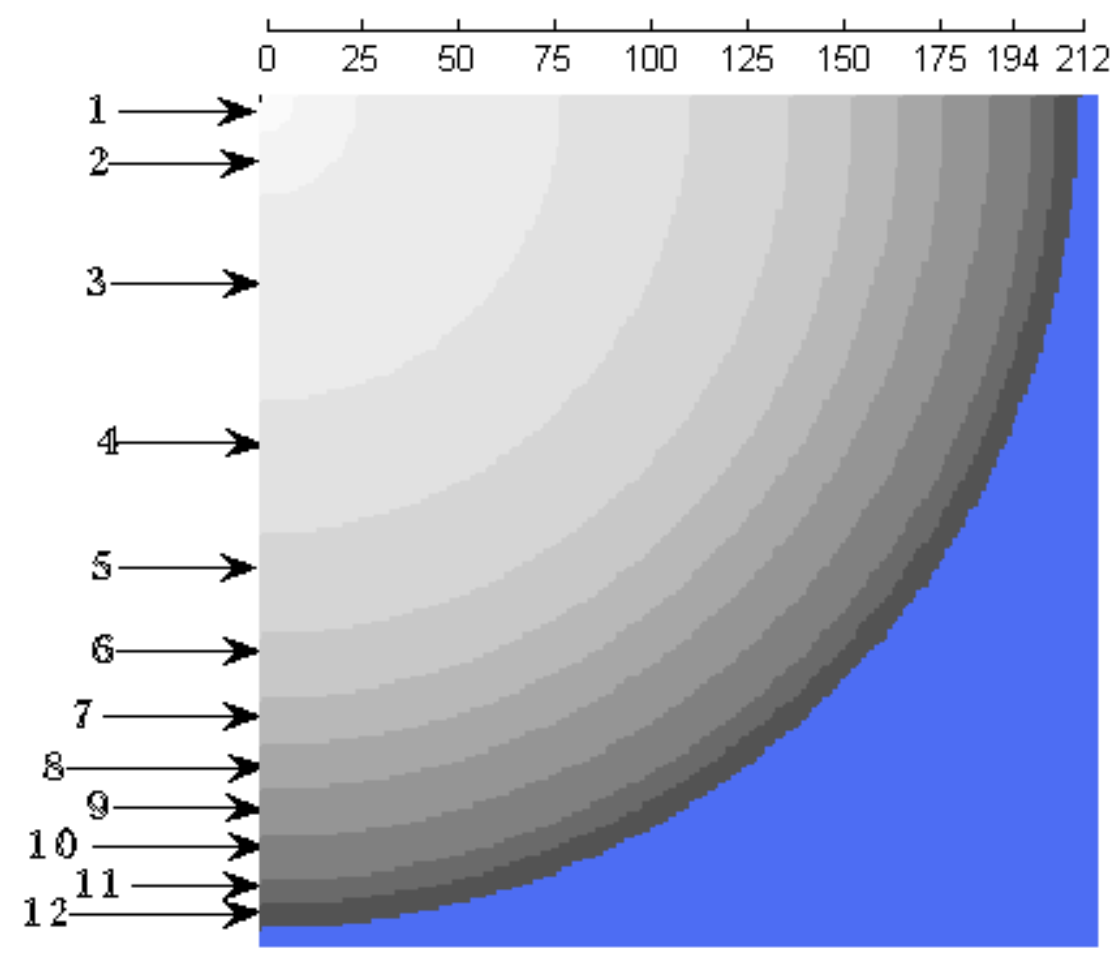

1: 4PAM, no code

2: 8PAM, RM(1,2)

3: 4PAM, no code

4: 4PAM, RM(1,2)

5: 2PAM, no code

6: $2 \mathrm{PAM}, \mathrm{RM}(1,2)$

7: 2PAM, RM(1,3)

8: 2PAM, RM(1,4)

9: 2PAM, RM(1,5)

10: 2PAM, RM(1,6)

11: 2PAM, RM(1,7)

12: 2 PAM, RM(1,8)

Figure 6.3: Frequency Regions Displaying Variable Modulation Schemes and Inner Code Rates: Red/Blue Channel

provide for more efficient decoding.

Because decoding complexity is a concern on mobile phones, an accumulate-repeataccumulate (ARA) code was chosen as the outer code. ARA codes can be viewed as serial turbo-like codes and offer high-speed iterative decoding implementations that are more practical for mobile phone processors than most other codes of similar performance [118]. The variable-rate inner code is a first-order Reed Muller (RM) code which has an efficient decoding algorithm based on the Fast Hadamard Transform. The barcode is split into regions based on radial distance from DC, and each region is encoded with a different rate RM code. Figures 6.3 and 6.4 display the resultant DCT regions for each colour channel.

While capacity estimates indicated that an overall code rate of $1 / 3$ would be required, the inner/outer rate breakdown was determined experimentally. Different inner rate and outer rate combinations were tested with the simulator. Outer ARA code rates of $(n+1 / n+2)$ for $n=0,1, \ldots, 7$ were tested with the inner rates adjusted accordingly. It was found that 


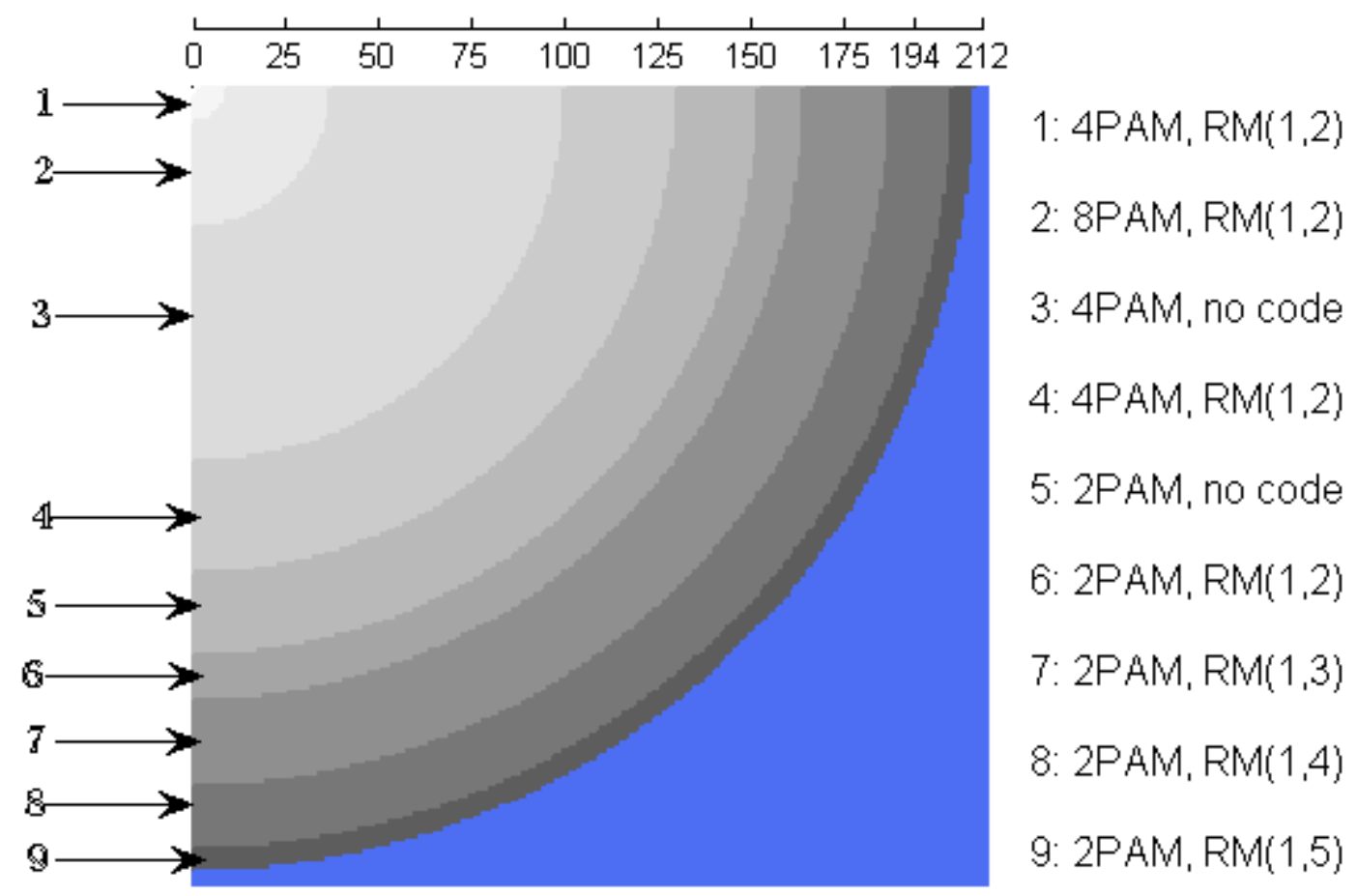

Figure 6.4: Frequency Regions Displaying Variable Modulation Schemes and Inner Code Rates: Green Channel 
a rate $1 / 2$ ARA code with an overall inner code rate of $2 / 3$ performed the best. (Allowing the more powerful ARA code to do more of the error correction appears to be a preferable allocation of resources, which is consistent with what was expected prior to simulation.)

\subsection{Modulation and Demodulation}

Because the DCT has only a real component, the modulation method used is pulse-amplitude modulation (PAM). Therefore, each particular sequence of bits is assigned to a corresponding symbol in a constellation of varying amplitude values. For a two-level constellation, this corresponds to symbols $\epsilon\{-A, A\}$ where $\mathrm{A}$, the amplitude, is equal to the square-root of the power value for that bin.

An appropriate PAM constellation requires defining a constellation size, or the number of channel bits per symbol. Given the capacity in each frequency bin, there is an upper bound on the number of data bits per symbol that can be transmitted reliably. Higher performance is achieved when the number of channel bits per symbol exceeds the number of information bits per symbol by roughly one bit, and a good error-correcting code is implemented. Because the capacity varies throughout the barcode, one constellation size will not accommodate every DCT bin properly, and therefore mapping must be done according to set frequency regions.

\subsubsection{Modulation and Mapping}

Modulation is the process of varying waveforms such that they can convey the message to be transmitted. For the DCT barcode, modulation is achieved by varying the amplitude in each frequency bin (PAM). This allows the receiver to interpret what was sent by analyzing the amplitudes in each bin. Because the receiver will be making decisions based on the received amplitudes, the gain control discussed in Section 5.2.7 is a critical step.

Based on the mutual information calculations discussed in Section 5.3, the barcode is split into twenty regions in the DCT domain (see Figure 6.3 and 6.4). Because the green channel has higher capacity in the RGB barcode, it is addressed separately from the red and blue. The regions are defined by their minimum and maximum radii from DC and the constellation size is $2^{b+1}$ rounded to the nearest power of two, where $b$ is the target information bit rate per symbol.

Once the number of bits / symbol has been defined for a particular region, the amplitude magnitudes must be assigned. In order to easily assign the different power values in each bin (according to the water-filling process defined in Section 5.1.3), the constellations are 
defined to have unit power. (That is, $E\left(|A|^{2}\right)=1$ over the constellation.) All constellations are symmetric about the origin in order to minimize the power [119]. Each symbol is multiplied by the square root of the power value designated for that bin. That is, the symbol in bin $(i, j)$ is multiplied by $\sqrt{P(i, j)}$ prior to transmission where $P(i, j)$ is the water-filling power assigned to bin $(i, j)$.

Once the bits have been coded, they are interleaved before mapping takes place. Interleaving is a method of changing the order of the bits according to an interleaver table. This allows them to be restored to their original order using the same table.

The bits are interleaved before being mapped to their respective constellations in order to avoid mapping bits of the same codeword to the same symbol, or neighbouring symbols which are assumed to be correlated. This will provide greater dispersion of errors across the region of interest, leading to a higher degree of performance at the decoder. If the inner code has rate 1 such that no inner coding is performed (i.e. each bit from the outer encoder is passed directly through the inner encoder for the region of interest), then interleaving is an unnecessary step that can be skipped for the region in the interest of efficiency at the decoder. A simple linear interleaver table is used, as it demonstrated better performance than a random interleaver for this particular purpose.

Bits are mapped into their respective constellations in the DCT domain according to a particular convention. Consider a 4-level constellation. The following illustrates different methods of mapping each 2-bit sequence to the four symbols in the constellation (distinguished by their amplitude):

$$
\begin{aligned}
& 01 \rightarrow-3 A, 00 \rightarrow-A, 01 \rightarrow A, \quad 11 \rightarrow 3 A(\text { Gray Method }) \\
& 11 \rightarrow-3 A, 00 \rightarrow-A, \quad 01 \rightarrow A, \quad 10 \rightarrow 3 A(\text { Arbitrary }(\text { very poor }) \text { Method }) \\
& 11 \rightarrow-3 A, \quad 01 \rightarrow-A, \quad 10 \rightarrow A, \quad 00 \rightarrow 3 A(\text { Natural Method })
\end{aligned}
$$

A Gray-mapping method, in which neighbouring symbols in the constellation differ in only one bit location, performed the best in simulation trials and is therefore the convention used. The receiver requires knowledge of the constellations in each region in order to demap and demodulate.

\subsubsection{Demodulation and De-Mapping}

At the receiver, once the gain control algorithm has been performed on each region, the symbols received are converted to soft-valued bit 'likelihoods', de-interleaved, and fed to the decoded. A log-likelihood ratio (or LLR), defined by

$$
\operatorname{LLR}(b)=\ln \left(\frac{\operatorname{Prob}(b=1)}{\operatorname{Prob}(b=0)}\right)
$$


conveys the confidence the receiver has in a particular bit being a one or a zero. This method of representing a received bit allows the decoder to use all the available information and to give more credibility to high-magnitude LLRs than those closer to zero.

The LLRs are calculated using a maximum-a-priori approach based on the received symbol, $R$, as described by

$$
\operatorname{LLR}(b)=\ln \left(\frac{\operatorname{Prob}(b=1 \mid R)}{\operatorname{Prob}(b=0 \mid R)}\right) .
$$

Note that the convention of $\operatorname{Prob}(b=1 \mid R) / \operatorname{Prob}(b=0 \mid R)$ is arbitrary and can be flipped to $\operatorname{Prob}(b=0 \mid R) / \operatorname{Prob}(b=1 \mid R)$.

Equation 6.2 can be simplified such that only the conditional probabilities of $R$ given $b$ need be calculated:

$$
\begin{gathered}
L L R(b)=\ln \left(\frac{\operatorname{Prob}(R \mid b=1) \operatorname{Prob}(b=1) / \operatorname{Prob}(R)}{\operatorname{Prob}(R \mid b=0) \operatorname{Prob}(b=0) / \operatorname{Prob}(R)}\right) \\
L L R(b)=\ln \left(\frac{\operatorname{Prob}(R \mid b=1)(1 / 2)}{\operatorname{Prob}(R \mid b=0)(1 / 2)}\right) \\
L L R(b)=\ln \left(\frac{\operatorname{Prob}(R \mid b=1)}{\operatorname{Prob}(R \mid b=0)}\right) .
\end{gathered}
$$

In order to calculate the probabilities, $\operatorname{Prob}(R \mid b)$, for bit $b$, the receiver assumes an additive white Gaussian noise (AWGN) channel of a particular noise variance, $\sigma_{N}^{2}$. The probabilities corresponding to every bit- 1 in the constellation are summed and divided by the sum of the probabilities corresponding to every bit- 0 in the constellation.

For example, in a 4-level constellation (4PAM) that assumes Gray-mapping such that $\{10,11,01,00\} \rightarrow\{-3 A,-A, A, 3 A\}$, the LLRs for the most significant and least significant bits are given by

$$
L L R(M S B)=\ln \left(\frac{\exp \left(-\left((3 * A+R)^{2}\right) /\left(2 \sigma_{N}^{2}\right)\right)+\exp \left(-\left((A+R)^{2}\right) /\left(2 \sigma_{N}^{2}\right)\right)}{\exp \left(-\left((-3 A+R)^{2}\right) /\left(2 \sigma_{N}^{2}\right)\right)+\exp \left(-\left((-A+R)^{2}\right) /\left(2 \sigma_{N}^{2}\right)\right)}\right)
$$

and

$$
\operatorname{LLR}(L S B)=\ln \left(\frac{\exp \left(-\left((A+R)^{2}\right) /\left(2 \sigma_{N}^{2}\right)\right)+\exp \left(-\left((-A+R)^{2}\right) /\left(2 \sigma_{N}^{2}\right)\right)}{\exp \left(-\left((-3 A+R)^{2}\right) /\left(2 \sigma_{N}^{2}\right)\right)+\exp \left(-\left((3 A+R)^{2}\right) /\left(2 \sigma_{N}^{2}\right)\right)}\right)
$$

respectively.

For a 2-level constellation size, the LLR calculation reduces to $-2 A R / \sigma_{N}^{2}$ which is easily performed on a mobile phone processor. For higher order constellations, the computation is more complex and will likely be approximated for real-time processing on mobile phones.

In order to demodulate the symbols in each barcode region and forward the resultant LLRs to the inner decoder, the receiver must have knowledge of the noise variances in each bin. These values are given by the measurements outlined in Appendix E. The receiver must also have knowledge of the constellations in each region and the mapping convention. 
Note that the LLRs in coded regions are de-interleaved prior to being sent to the inner decoder.

\subsection{Inner Reed Muller Code}

Reed Muller codes, invented in 1954 by D. E. Muller and I. S. Reed, are a well-known family of linear error-correcting block codes [120]. Reed Muller codes are relatively easy to decode, and first-order codes are especially efficient, which is why they were chosen to be the inner codes. The variable rates are given according to Figures 6.3 and 6.4.

\subsubsection{Inner Encoding}

An $r^{t h}$ order $((r, m))$ Reed Muller code is the set of all binary vectors of length $2^{m}$ associated with the Boolean polynomials $p\left(x_{1}, x_{2}, \ldots, x_{m}\right)$ of degree less than or equal to $r$ [120]. First order Reed Muller codes are $\left(2^{m}, k, 2^{m-1}\right)$ codes, where $k=m+1$.

Encoding is achieved by multiplying each $2^{k}$ binary string by the $k \times 2^{m}$ generator matrix. To form a first-order $((1, m))$ Reed Muller code, a generator matrix is constructed by adding vectors $x_{1}, x_{2}, \ldots, x_{m}$ below a row vector of $2^{m}$ ones. The generator matrices for $(1,1),(1,2)$, and $(1,4) \mathrm{RM}$ codes are given by

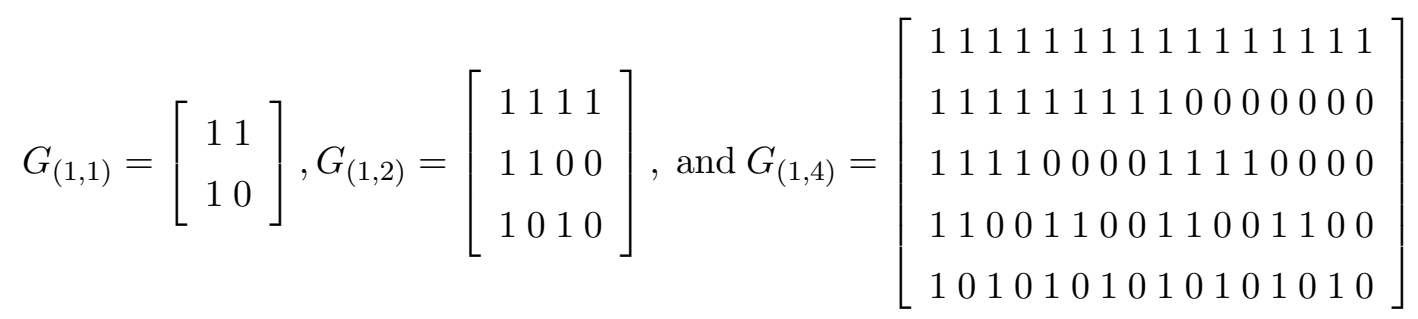

respectively.

Each of the DCT regions is encoded with a designated Reed Muller code. Figures 6.3 and 6.4 illustrate the inner coding breakdown within the barcode.

To take advantage of the efficient maximum a-posteriori decoding method of Ashikhmin and Litsyn ([121]), discussed further in Section 6.2.2, a systematic first-order Reed Muller code is used - meaning that the $k$ information bits are transmitted in addition to $2^{m}-k$ parity bits.

Note that for the general first-order RM generator matrices, the code is non-systematic (all code bits are parity bits). For the systematic RM code used, the information bits are transmitted directly in the codeword. However, for reasons that will become more apparent in Section 6.2.2, the systematic bits are dispersed in the codeword such that the set of 
$\mathrm{RM}(1, \mathrm{~m})$ codewords form the columns of the $2^{m} \times 2^{m}$ Hadamard matrix, described by

$$
\begin{gathered}
H_{1}=[1] \\
H_{2^{m}}=\left[\begin{array}{cc}
H_{2^{m-1}} & H_{2^{m-1}} \\
H_{2^{m-1}} & -H_{2^{m-1}}
\end{array}\right],
\end{gathered}
$$

and its complement. (Note that this is also the case for the non-systematic $\operatorname{RM}(1, \mathrm{~m})$ code.)

For the systematic version, the first three information bits become the first three code bits, and the $(n+3)^{t h}$ information bit becomes the $\left(2+\sum_{i=0}^{n} 2^{i}\right)^{t h}$ code bit for $n=0,1, \ldots, k-3$. The codeword is given by the corresponding column (or column complement) in $H_{2^{m}}$. (For the rate $1 \mathrm{RM}(1,1)$ case, only the information bits are transmitted.) The generator matrices for $k=2,3$, and 4 are

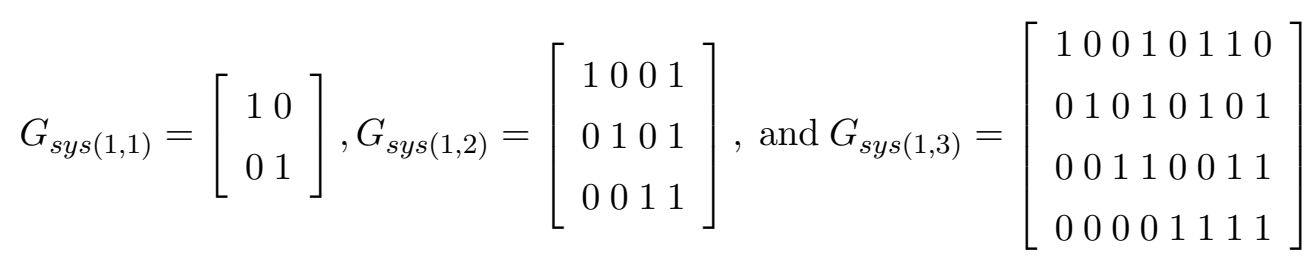

respectively.

Instead of generating codewords by multiplying the $k$ information bits by the corresponding $k \times 2^{m}$ generator matrix, one can simply transmit the column of $H_{2^{m}}$ (with $-1 \mathrm{~s}$ replaced by $0 \mathrm{~s}$ ) corresponding to the information bits. (If the information bits match the $1^{s t}, 2^{\text {nd }}, \ldots,\left(2+\sum_{i=0}^{k-3} 2^{i}\right)^{t h}$ entries of the columns of $\pm H_{2^{m}}$, that column/column complement is transmitted.)

Using the newly defined systematic code, the codewords still form the columns of $\pm H_{2^{m}}$ ， and the information bits are transmitted directly in the codeword, which will allow for very efficient soft-in-soft-out (SISO) decoding.

\subsubsection{Inner Decoding}

Maximum-likelihood (ML) decoding of Reed-Muller codes is based on the distance between vectors, where distance is defined to be the number of places that differ between two vectors. The original encoded message is assumed to be that corresponding to the codeword of least distance from the received vector. Because the distance between any two codewords in the $(1, \mathrm{~m}) \mathrm{RM}$ code is $2^{m-1}, 2^{m-1}-1$ errors can be corrected in any given received word.

Some efficient algorithms have been developed for first-order Reed-Muller codes. The fast Hadamard transform $\left(F H T_{R}\right)$ provides a particularly efficient method. As previously discussed, the codewords of an $\operatorname{RM}(1, \mathrm{~m})$ code are the columns of $\pm H_{2^{m}}$. Therefore, the 
received word can be correlated against each column of the $H_{2^{m}}$ Hadamard matrix to determine the maximum-likelihood codeword transmitted. That is, the highest magnitude inner product over all columns in $\pm H_{2^{m}}$ with the received word indicates which column, $h_{j}$ was transmitted. If the inner product is less than zero, $-h_{j}$ is the chosen codeword (and otherwise $h_{j}$ is) [122].

Because the outer code requires LLRs as an input, a soft-input soft-output (SISO) inner decoder is employed. The decoding method is the maximum a-posteriori (MAP) method of Ashikhmin and Litsyn. The MAP SISO method allows for higher performance decoding than the hard decision ML method, and remains efficient due to its use of the $F H T_{R}$.

The MAP decoding method calculates the LLRs for each transmitted code bit. Because the RM code employed is systematic, as discussed in Section 6.2.1, the embedded information bit LLRs are then obtained immediately. The code bit LLRs are given by

$$
\ln \left(\frac{\operatorname{Pr}\left(c_{j}=1 \mid \vec{y}\right)}{\operatorname{Pr}\left(c_{j}=0 \mid \vec{y}\right)}\right),
$$

where $\vec{y}$ denotes the received codeword and $c_{j}$ denotes the $j^{\text {th }}$ corresponding code bit. Therefore, the following must be computed for each transmitted codeword, $\vec{c}=\left(c_{0}, c_{1}, \ldots\right.$, $\left.c_{2^{m}-1}\right) \in C$, based on the corresponding received word, $\vec{y}$ :

$$
\begin{aligned}
\operatorname{Pr}\left(c_{j}=\alpha \mid \vec{y}\right) & =\sum_{\vec{x} \in C_{j}^{\alpha}} \operatorname{Pr}(\vec{c}=\vec{x} \mid \vec{y}) \quad \text { for } \alpha \in\{0,1\} \\
& =\sum_{\vec{x} \in C_{j}^{\alpha}} \frac{\operatorname{Pr}(\vec{y} \mid \vec{x})}{\operatorname{Pr}(\vec{y})} \operatorname{Pr}(\vec{x}) \\
& =\frac{1}{2^{k} \operatorname{Pr}(\vec{y})} \sum_{\vec{x} \in C_{j}^{\alpha}} \prod_{j=0}^{2^{m}-1} \operatorname{Pr}\left(y_{j} \mid x_{j}\right)
\end{aligned}
$$

where $C_{j}^{\alpha}=\left\{\vec{c}=\left(c_{0}, c_{1}, \ldots, c_{2^{m}-1}\right) \in R M(1, m): c_{j}=\alpha\right\}$ (i.e. $C_{j}^{\alpha}$ is the set of all codewords in $C$ whose $j^{\text {th }}$ bit is $\alpha$ ).

The MAP decoding algorithm uses the $F H T_{R}$, which allows for $\left(s_{0}, s_{1}, \ldots, s_{2^{m}-1}\right)=\left(v_{0}\right.$, $\left.v_{1}, \ldots, v_{2^{m}-1}\right) \cdot H_{2^{m}}$ to be computed in $O\left(n \log _{2} n\right)$ (where $n=2^{m-1}$ ) instead of $O\left(n^{2}\right)$. To compute the $\mathrm{FHT}_{R}$ of $\vec{v}$, the following steps are performed $\log _{2} n$ times:

$$
\begin{aligned}
\text { for } j & =0 \text { to } 2^{m-1} \text { compute }: \\
s_{j} & =v_{2 j}+v_{2 j+1}, \quad s_{2^{m-1}+j}=v_{2 j}-v_{2 j+1} \\
\text { for } j & =0 \text { to } 2^{m}-1 \text { assign } v_{j}=s_{j} .
\end{aligned}
$$

This transform will prove to be very useful in the decoding process. A brief description of the decoding method is given here and more detail can be found in [121].

To begin, let $\vec{c}=\left(c_{0}, c_{1}, \ldots, c_{2^{m}-1}\right) \in C$ denote the transmitted codeword and $\vec{y}$ be the received vector. Let $\vec{b}$ represent the vector of corresponding LLRs, where $b_{i}=\ln \left(\operatorname{Pr}\left(y_{i} \mid 1\right) /\right.$ $\left.\operatorname{Pr}\left(y_{i} \mid 0\right)\right)$ for $i=1,2, \ldots, 2^{m}$. Let $C_{j}^{\alpha}=\left\{\vec{c}=\left(c_{0}, c_{1}, \ldots, c_{2^{m}-1}\right) \in R M(1, m): c_{j}=\alpha\right\}$ so that $C_{j}^{\alpha}$ is the set of all codewords in $C$ whose $j^{\text {th }}$ bit is $\alpha$ for $\alpha \in\{0,1\}$. 
The entries of the vector $\vec{t}=\vec{b} \cdot H_{2^{m}}^{\perp}$ are equal to the expression

$$
t_{i}=\sum_{j=1}^{2^{m}} \ln \left(\operatorname{Pr}\left(y_{j} \mid c_{i j}\right)\right)-\sum_{j=1}^{2^{m}} \ln \left(\operatorname{Pr}\left(y_{j} \mid c_{i j} \oplus 1\right)\right), \quad i=0, \ldots, 2^{m}-1
$$

and can be computed using the $F H T_{R}$, where $\oplus$ represents binary addition and $c_{i j}$ represents $j^{\text {th }}$ bit of the $i^{\text {th }}$ codeword in $C$ (so $c_{i j} \oplus 1$ is the $j^{\text {th }}$ bit of the $i^{\text {th }}$ codeword complement, or simply the $\left(i+2^{m}\right)^{t h}$ codeword in $C$ ). Note that $\overrightarrow{c_{i}}$ denotes the $i^{\text {th }}$ codeword in $C$ and $\overrightarrow{c_{i}} \oplus 1$ is its complement.

The sums of logarithms of probabilities can be computed from the differences of the sums as follows: Let $s=\sum_{j=1}^{2^{m}} \ln \left(\operatorname{Pr}\left(y_{j} \mid 0\right)\right)+\ln \left(\operatorname{Pr}\left(y_{j} \mid 1\right)\right)$, where $\operatorname{Pr}\left(y_{j} \mid 0\right)$ and $\operatorname{Pr}\left(y_{j} \mid 1\right)$ are readily obtained from $b_{i}$. Then Equations 6.11 and 6.12 can be used to compute to compute $\operatorname{Pr}\left(\vec{y} \mid \overrightarrow{c_{i}}\right)$.

$$
\begin{gathered}
r_{i}^{(0)}=\sum_{j=1}^{2^{m}} \ln \left(\operatorname{Pr}\left(y_{j} \mid c_{i j}\right)\right) \\
r_{i}^{(1)}=\sum_{j=1}^{2^{m}} \ln \left(\operatorname{Pr}\left(y_{j} \mid c_{i j} \oplus 1\right)\right) \\
v_{i}^{(0)}=e^{r_{i}^{(0)}}=\prod_{j=0}^{2^{m}-1} \operatorname{Pr}\left(y_{j} \mid c_{i j}\right)=\operatorname{Pr}\left(\vec{y} \mid \overrightarrow{c_{i}}\right) \\
v_{i}^{(1)}=e^{r_{i}^{(1)}}=\prod_{j=0}^{2^{m}-1} \operatorname{Pr}\left(y_{j} \mid c_{i j} \oplus 1\right)=\operatorname{Pr}\left(\vec{y} \mid \overrightarrow{c_{i}} \oplus 1\right)
\end{gathered}
$$

Now the soft decisions, $\operatorname{Pr}\left(c_{j}=1 \mid \vec{y}\right)-\operatorname{Pr}\left(c_{j}=0 \mid \vec{y}\right)$ can be calculated. Let $x_{i}=$ $\operatorname{Pr}\left(\vec{y} \mid \overrightarrow{c_{i}}\right)-\operatorname{Pr}\left(\vec{y} \mid \overrightarrow{c_{i}} \oplus 1\right)$. Since $C$ contains the all-one codeword, the entries of the vector $\vec{w}$, where $\left(w_{0}, w_{1}, \ldots, w_{2^{m}-1}\right)=\left(x_{0}, x_{1}, \ldots, x_{2^{m}-1}\right) \cdot H_{2^{m}}$, are given by

$$
\begin{aligned}
w_{j} & =\sum_{i=0}^{2^{m}-1} h_{i j}\left(\operatorname{Pr}\left(\vec{y} \mid \overrightarrow{c_{i}}\right)-\operatorname{Pr}\left(\vec{y} \mid \overrightarrow{c_{i}} \oplus 1\right)\right) \quad j=0,1, \ldots, 2^{m}-1 \\
& =\sum_{\vec{c} \in C_{j}^{(0)}} \operatorname{Pr}(\vec{y} \mid \vec{c})-\sum_{\vec{c} \in C_{j}^{(1)}} \operatorname{Pr}(\vec{y} \mid \vec{c}) \quad j=0,1, \ldots, 2^{m}-1
\end{aligned}
$$

in which $h_{i j}$ denotes the $(i, j)$ th entry of $H_{2^{m}}$ [121]. The vector $\vec{w}$ is computed efficiently by taking the $F H T_{R}$ of $\vec{x}$, where $x_{i}=v_{i}^{(1)}-v_{i}^{(0)}$.

Finally, the scale factor from Equation 6.9, $q=2^{m+1} \operatorname{Pr}(\vec{y})$, is computed according to

$$
q=\sum_{j=1}^{2^{m}}\left(v_{j}^{(1)}+v_{j}^{(0)}\right) .
$$

Note that $\operatorname{Pr}\left(c_{j}=1 \mid \vec{y}\right)-\operatorname{Pr}\left(c_{j}=0 \mid \vec{y}\right)=\frac{\vec{w}}{q}$ such that $\operatorname{Pr}\left(c_{j}=1 \mid \vec{y}\right)=\frac{1}{2}\left(\overrightarrow{1}+\frac{\vec{w}}{q}\right)$ and $\operatorname{Pr}\left(c_{j}=0 \mid \vec{y}\right)=\frac{1}{2}\left(\overrightarrow{1}-\frac{\vec{w}}{q}\right)$, where $\overrightarrow{1}$ is a $1 \times 2^{m}$ vector of all ones. This allows for the final LLR vector to be computed as

$$
L_{\text {out }}=\ln \left(\frac{\operatorname{Pr}\left(c_{j}=1 \mid \vec{y}\right)}{\operatorname{Pr}\left(c_{j}=0 \mid \vec{y}\right)}\right)=\ln \left(\frac{\overrightarrow{1}+\frac{\vec{w}}{q}}{\overrightarrow{1}-\frac{\vec{w}}{q}}\right) .
$$




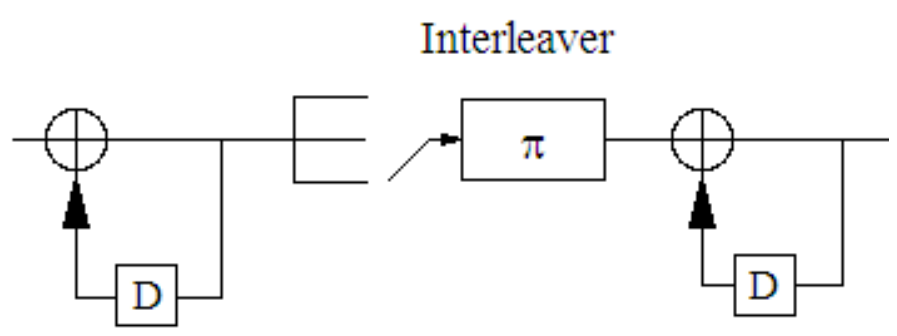

Accumulator

Figure 6.5: A Basic ARA Encoding Structure

The information bit LLRs are then taken directly from the corresponding systematic bit locations in $\overrightarrow{L_{\text {out }}}$.

Therefore, the overall inner SISO Reed-Muller decoding algorithm is as follows (refer to [121] for more information):

$$
\begin{aligned}
& \text { input }=\text { channel LLRs from demodulator } \\
& \text { for } i=1: \text { no. codewords in block } \\
& \quad \text { let LLRs for codeword } i=\vec{b} \\
& \quad \text { compute } \vec{t}=F H T_{R}(\vec{b}) \\
& \quad \text { compute } \overrightarrow{v^{(0)}} \text { and } \overrightarrow{v^{(1)}} \text { according to Equation } 6.12 \\
& \quad \text { compute } \vec{w}=F H T_{R}\left(\overrightarrow{v^{(1)}}-v^{(0)}\right) \\
& \quad \text { compute scale factor } q \text { according to Equation } 6.14 \\
& \quad \text { final LLR vector for codeword } i=\ln ((\overrightarrow{1}+\vec{w} / q) /(\overrightarrow{1}-\vec{w} / q)) \\
& \quad \text { take the } k \text { systematic code bits to be the final } L L R s \text { for word } i \\
& \text { end } \\
& \text { output the inner bit LLRs to the outer decoder }
\end{aligned}
$$

Once the inner decoding is complete, the outer decoding takes place. Note that for the regions in which no inner code is applied, the channel LLRs from the demodulator are passed directly to the outer decoder.

\subsection{Outer ARA Code}

ARA codes were introduced in 2007 by A. Abbasfar, D. Divsalar, and K. Yao, and shown to closely approach the performance of random codes. The ARA encoder concept is that of a repeat accumulate (RA) encoder preceded by an accumulator (the precoder) as seen in Figure 6.5. 


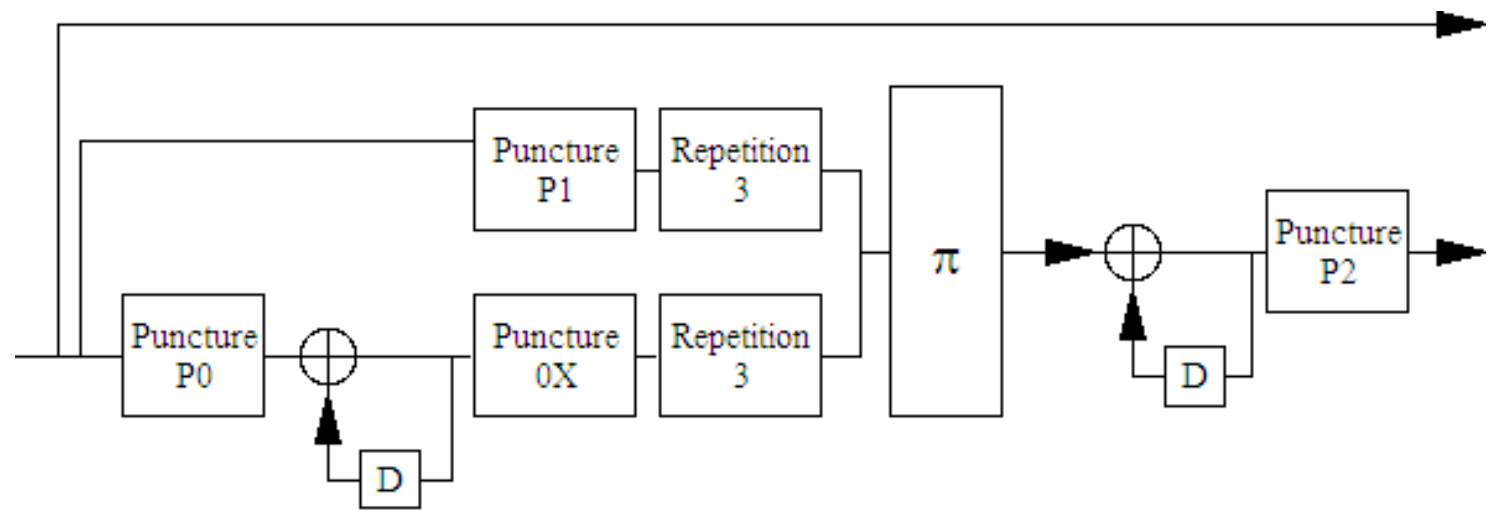

Figure 6.6: Family of Multi-edge Type ARA Codes by Abbasfar et al. (after [118])

The pre-coding gain achieved for a systematic RA code with an outer accumulator is discussed by A. Abbasfar et al. in [118], and they go on to present a more sophisticated and effective family of ARA codes. This family of codes is discussed further in the following section.

\section{ARA Structure}

Abbasfar et al. present family of systematic, irregular, multi-edge type ARA codes with an encoder structure illustrated in Figure 6.6. Some portion of the input bits is not passed through the precoding accumulator due to more puncturing of the inner accumulator. This allows for the iterative decoding to start - a technique called doping [118, 123].

The corresponding protograph is illustrated in Figure 6.7 and conveys the multi-edge code structure. For standard irregular codes, graph connectivity is constrained only by the node degrees. For multi-edge structures, several edge (equivalence) classes are defined and each node is characterized by its number of sockets in each class. The key is that only sockets of the same class can be connected by an edge [124].

Consider the protograph of Figure 6.7. The nodes with a plus sign inside are check nodes and the others are variable nodes. The solid black variable nodes are transmitted, while the hollow (white) variable nodes are not (i.e. they are punctured nodes). Check nodes 1 and 2 essentially represent the inner accumulator. It can be seen that variable node 1, connected to the pre-accumulated repeated nodes, and variable nodes 2 through $2 n+1$, connected to the non-accumulated repeated nodes, are connected to check nodes 1 and 2 in a particular order.

The interleaving is done using six interleavers, one for each edge class between the repetition 3 and the punctured accumulator. For the rate 1/2 code used in the final design, 


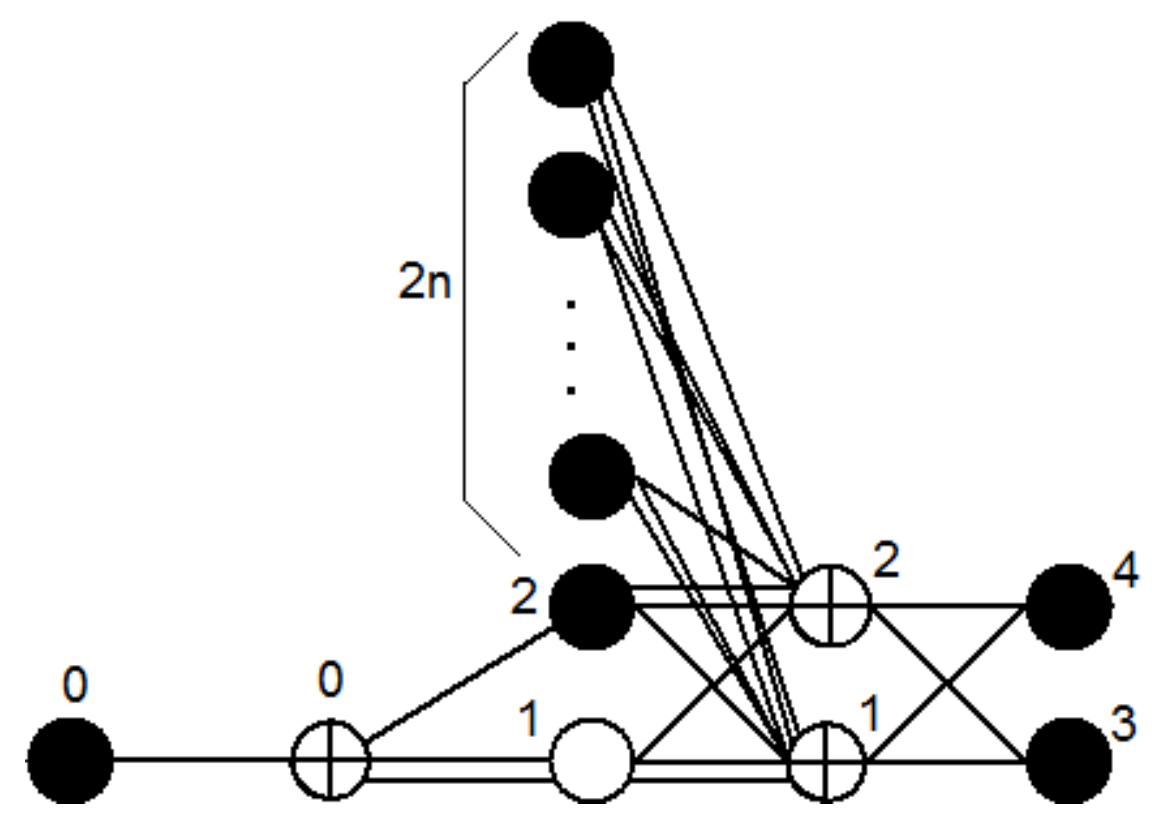

Figure 6.7: Protograph for Multi-Edge Family of ARA Codes of Rate $=(n+1) /(n+2)($ after [118])

six interleavers of size $\mathrm{N} / 2$, where $\mathrm{N}$ is the input block size, are used (see factor graph in Figure 6.8). This corresponds to the rate 1/2 protograph seen in Figure 6.9.

Consider the factor graph in Figure 6.8. The non-accumulated repeated LLRs entering the interleaver from above (on the left) are assigned to classes 1, 2, and 3. The accumulated repeated bits entering the interleaver from above (on the right) are assigned to classes 4, 5, and 6. The LLRs entering the interleaver from below are numbered 4, 5, 1, 6, 2, 3, corresponding to the six classes. Figure 6.10 displays the ordering on the factor graph. In this manner, the edge structure of Figure 6.9 is adhered to.

\section{Interleaving Strategy}

The interleaving strategy is a critical factor in the performance of the code. Although it is not obvious from the encoder structure seen in Figure 6.6, managing the edge classes with multiple interleavers crucial to achieving performance targets laid out by Abbasfar et al. In addition, the type of interleaver used is an important design parameter. According to Abbasfar et al., circulant permutations are preferred to random interleaving. However, in the interest of time, well-established interleaving strategies were investigated.

Four different interleavers were tested: a random interleaver, a golden interleaver, a linear interleaver, and a golden dithered interleaver. The random interleaver and the dithered golden interleaver by Crozier et al. performed the best and had roughly the same perfor- 


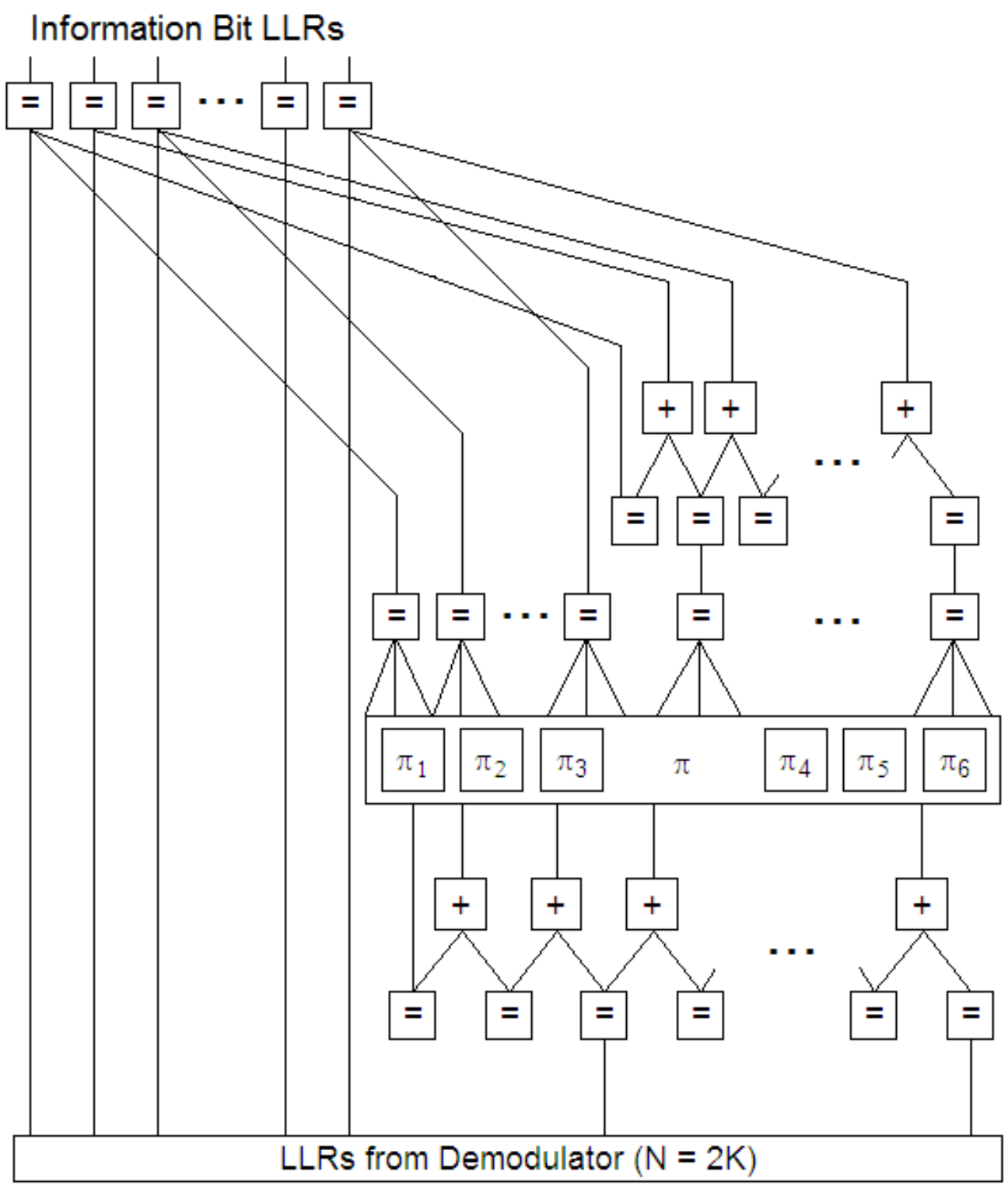

Figure 6.8: Factor Graph for Rate 1/2 Multi-Edge ARA Code

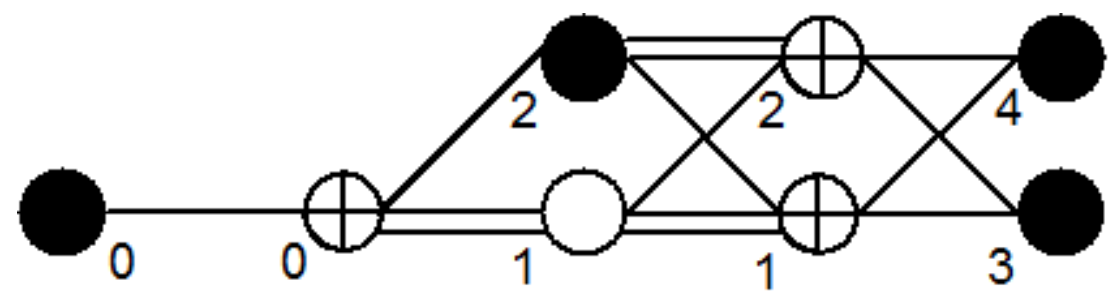

Figure 6.9: Protograph for Rate 1/2 Multi-Edge ARA Code (after [118]) 


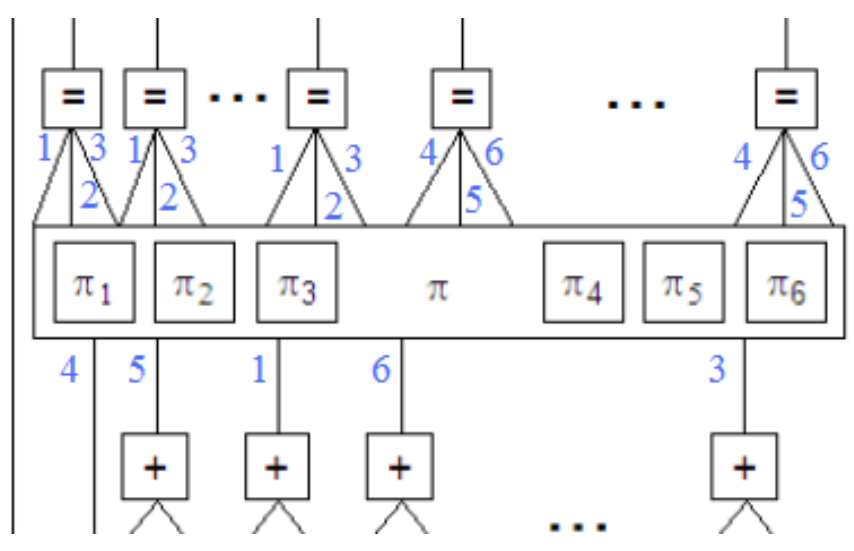

Figure 6.10: Interleaver from Figure 6.8 with Edge Classes Numbered

mance to Abbasfar et al. for lower SNR values (but hit a bit error rate (BER) floor just below 1.0E-06). The dithered golden interleaver is easily generated and therefore practical for mobile phone applications, unlike storing a pseudo-random interleaver in memory.

Golden interleavers are based on sorting real-valued numbers derived from the golden section [125]. Dithered golden interleavers are golden interleavers with a dither component included to add randomness, which improves Turbo code performance. Although the interleavers are designed for Turbo codes, they performed well for the ARA code, which can be viewed as a serial turbo-like code [118]. The algorithm for generating the interleaver table is provided below. The reader is referred to [125] for more information.

The first step in generating the dithered golden interleaver is to calculate the golden section value,

$$
g=\frac{\sqrt{5}-1}{2} \approx 0.618
$$

from which the real increment value,

$$
c=N(g m+j) / r
$$

is computed given an input block length, $N$. Note that $m$ is any integer greater than zero, $r$ is the index spacing between nearby elements to be maximally spread, and $j$ is any integer modulo $r$. Then a vector, $\vec{v}$, of real values is generated according to

$$
v(n)=s+n c+d(n), \operatorname{modulo} N, n=0 \ldots N-1,
$$

where $s$ is an integer starting value, and $d(n)$ is the $n^{\text {th }}$ dither component, uniformly distributed over $[0, N D]$ ( $\mathrm{D}$ is the normalized width of the dither distribution). Crozier et. al. specify $D \approx 0.01$ for Turbo codes of any block length [125]. Vector $\vec{v}$ is then sorted and the index vector that defines the sort is the interleaver index vector. 


\begin{tabular}{lcccc} 
Parameters: & $\mathrm{s}$ & $\mathrm{r}$ & $\mathrm{j}$ & $\mathrm{m}$ \\
\hline \hline & & & & \\
Interleaver 1: & 0 & 1 & 0 & 1 \\
Interleaver 2: & $(1 / 3) \cdot X$ & 8 & 1 & 2 \\
Interleaver 3 & $(2 / 3) \cdot X$ & 16 & 0 & 1 \\
Interleaver 4 & 0 & 4 & 0 & 2 \\
Interleaver 5 & $(1 / 3) \cdot Y$ & 12 & 3 & 1 \\
Interleaver 6 & $(2 / 3) \cdot Y$ & 20 & 5 & 2 \\
& & & & \\
where $X$ is the number of bits entering Interleavers 1, 2, 3 \\
and $Y$ is the number of bits entering Interleavers 4, 5, 6 \\
\hline \hline
\end{tabular}

Table 6.1: Golden Dithered Interleaver Parameters used for ARA Code

The last step in the process is to find the sort vector, $\vec{z}$, such that $a(n)=v(z(n))$ for $n=0 \ldots N-1$, where $\vec{a}=\operatorname{sort}(\vec{v})$. The interleaver indices are then given by $i(z(n))=n$ for $n=0 \ldots N-1$.

The parameters $(s, r, j$, and $m)$ of the six golden dithered interleavers used for the ARA code are given in Table 6.1.

\section{MAP Decoding}

Soft-input, soft-output maximum a posteriori (MAP) decoding is performed. The factor graph of Figure 6.8 applies. For each check node (denoted by a plus sign in the factor graph), the outgoing LLR, $L_{\text {out }}$, is given by

$$
L_{\text {out }}=2 \tanh ^{-1}\left(\tanh \left(L_{i n 1} / 2\right) \cdot \tanh \left(L_{i n 2} / 2\right)\right)
$$

and the approximation described by

$$
L_{\text {out }}=\max \left(L_{i n 1}, L_{i n 2}\right)-\max \left(0, L_{i n 1}+L_{i n 2}\right)
$$

is made to reduce complexity [124, 126]. The difference in the two functions is illustrated in Appendix F. There is a slight effect on the performance of the code, but the complexity trade-off makes the approximation worthwhile.

At each equality node (denoted by an equal sign in the factor graph), the outgoing LLR is given by the sum of all incoming LLRs. 
The decoding is terminated at 30 iterations or for a valid codeword. A 16-bit cyclicredundancy-check $(\mathrm{CRC})$ is added to the information bit stream before encoding. This allows the decoder to check whether a valid codeword has been found after a certain number of iterations, thus eliminating any further unnecessary iterations.

\section{Cyclic Redundancy Check}

Adding a Cyclic redundancy check (CRC) to a block of data is a very common method of providing for error-detection capability. The CRC is calculated by performing a modulo 2 division of the data by a generator polynomial and recording the remainder after division [127]. CRC-CCITT, from the Telegraph and Telephone Consultative Committee (CCITT) standards body, is a well-known and widely used 16-bit CRC. The CCITT polynomial is $x^{16}+x^{12}+x^{5}+1$. Because the polynomial is of degree 16 , the remainder of division by the polynomial is represented by 16 bits.

Before the data bit stream is sent to the outer ARA encoder, it is divided by the CCITT polynomial and the 16-bit remainder is appended to the data bits. At the decoder, after 8 iterations, hard decisions are made and the resulting stream is divided by the CCITT polynomial. if the remainder is not zero, the decoder knows that errors remain and another iteration commences. If the remainder is zero, the decoder infers that there are no errors left and terminates. This process continues until the maximum number of iterations (30) is reached, at which point the decoder declares an error and terminates.

The 16-bit CRC-CCITT is has an error-detection capability equal to $1-2^{-16}=0.999985$, meaning that it detects $99.9985 \%$ of all error bursts of length $>17$. It detects all error bursts of length 16 or less, and $99.997 \%$ of error bursts of length 17 [128]. 


\section{Chapter 7}

\section{Final Design and Results}

The final RGB DCT barcode design is summarized in Table 7.1 and displayed in Figure 7.1. The data density for the $404 \times 404$ pixel barcode is 0.285 bits/pixel (5.8 KB). At this density, the barcode were successfully decoded under the worst channels in the range of acceptable parameters. Under more ideal channel parameters, the density is estimated to be closer to 0.8 bits/pixel.

\subsection{Design Summary}

The complexity at the receiver is summarized in Table 7.2 for the $404 \times 404$ pixel barcode. The total estimated complexity is $2.07 \times 10^{8}$ arithmetic computations (plus some overhead). Note that the demodulation LLR calculations for 4PAM and 8PAM currently use floating point computations, but can be approximated (which may also reduce the complexity) for phones with fixed-point processing only. It can be assumed that for every arithmetic computation, there are approximately two reads and a write required. On a $400 \mathrm{MHz}$ processor, it is approximated that the entire decoding algorithm will take

$$
\frac{(2 \text { cycles/instr. }) \cdot(4 \text { instr./arith.instrc. }) \cdot(208 \text { M arith.instrc. })}{400 \text { M cycles/sec }}
$$

$\approx 4.16$ seconds.

Figure 7.2 illustrates a breakdown of the complexity at the receiver. It can be seen that acquisition dominates the number of computations. The acquisition method reduces the number of pixels being evaluated from $1600 \times 1200$ to $400 \times 400$ (a factor of 12 reduction). (The number of pixels is further reduced upon moving into the DCT domain.)

Essentially, because the receiver does not have any knowledge of where the barcode is in the received image, nor what relative size it is, a larger number of matched filter operations 
Processing:

Modulation

Transmitter

- Pre-Distortion (for CRT or LCD transmission)

- Noise-Shaping (POCs with 200 iterations)

Receiver

- Fast Coarse Acquisition using Bull's-Eye Fiducials

- Fine Acquisition

- Perspective Correction

- Spatial Domain Global Gain Adjustment (corrects Gray-World AWB)

- Gamma Correction (optional)

- Frequency Domain Gain Control (by radial frequency region)

and Mapping:

Code:

- SDMT using DCT basis functions

- PAM, constellation size varying by radial

frequency region (1-3 bits/symbol)

- Water-filling power allocation

- Power budget $=2267 /$ colour channel

- DC value $=\frac{1}{2} \cdot 400=200$

- Gray mapping

Overall Code Rate $\approx 1 / 3(0.3303)$

Outer Code

- Multi-edge Rate 1/2 ARA

- 6 Golden Dithered Interleavers, $\{s, r, j, m\}=$ $\{\{0,1,0,1\},\{(K / 6), 8,1,2\},\{(K / 3), 16,0,1\}$, $\{0,4,0,2\},\{(K / 6), 12,3,1\},\{(K / 3), 20,5,2\}\}$ ( $K=$ block length)

- Belief propagation MAP decoding (max 30 iterations)

Inner Code

- $1^{\text {st }}$ Order Reed Muller Code

- Rate Varying by Radial Frequency Region (rates $\frac{9}{256}-1$ )

- Fast MAP Decoding using $F H T_{R}$

Table 7.1: RGB DCT Barcode Design Specifications 


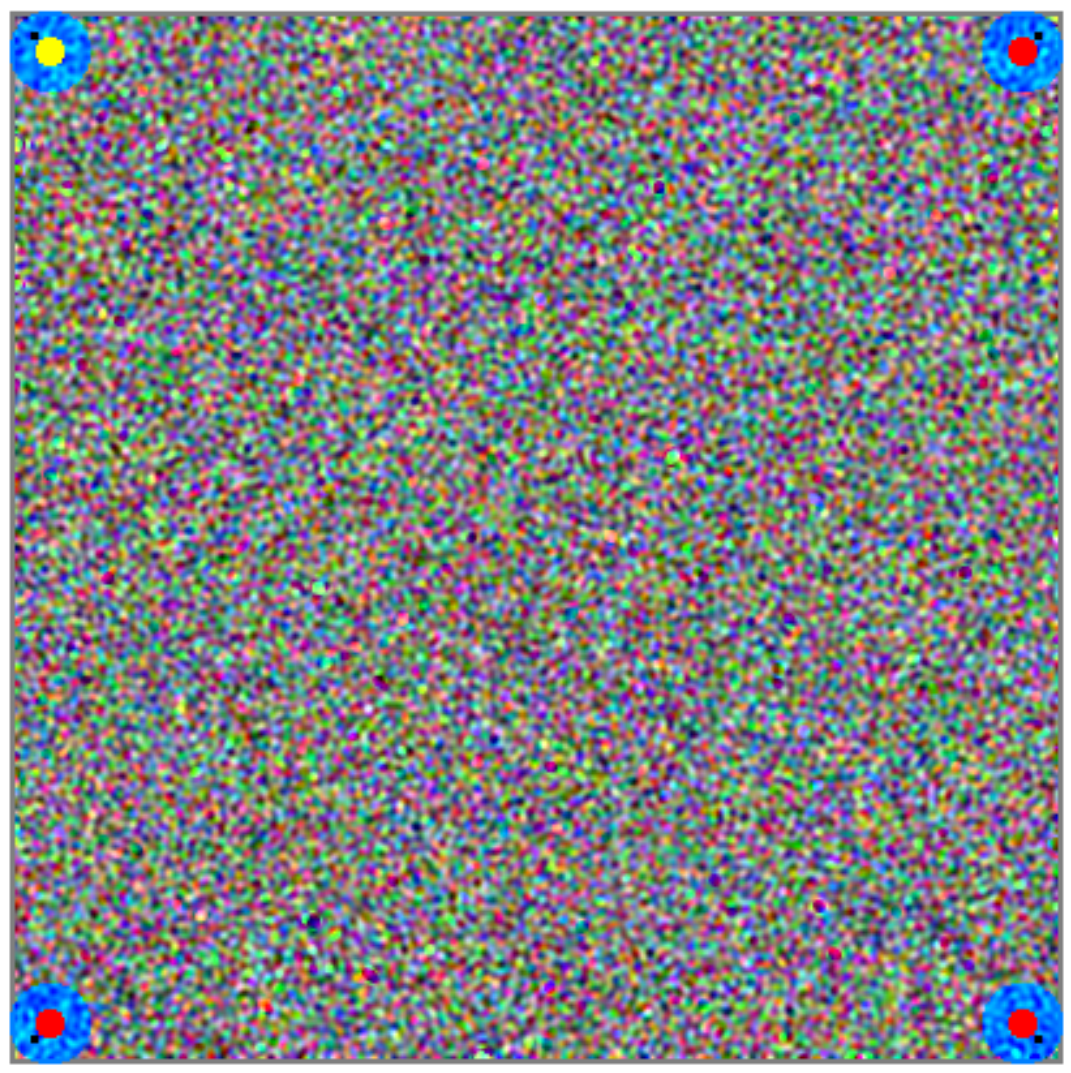

Figure 7.1: Final RGB DCT Barcode 


\begin{tabular}{|c|c|}
\hline Acquisition: & $\approx 130,000,000$ computations \\
\hline Perspective Correction: & $\begin{array}{l}\approx 8,800,000 \text { computations } \\
\quad(33 \text { computations per transmitted pixel })\end{array}$ \\
\hline Spatial Domain Gain Control: & $\begin{aligned} \approx & 480,000 \text { computations } \\
& (2 \text { computations per transmitted pixel })\end{aligned}$ \\
\hline 2-D DCT*: & $\begin{array}{l}\approx 7,323,000 \text { computations } \\
\quad 3 \cdot(\pi / 4) \cdot\left(4 \cdot 210^{2} \cdot \log _{2}\left(400^{2}\right)\right)\end{array}$ \\
\hline Frequency Domain Gain Control: & $\begin{array}{l}\approx 627,048 \text { computations } \\
\quad(3 \text { computations per symbol })\end{array}$ \\
\hline Demodulation and De-Mapping: & $\begin{array}{l}\approx 1,983,994 \text { computations } \\
\quad(\approx 4 / \text { symbol in } 2 \mathrm{PAM}, \approx 60 / \text { symbol in } \\
\quad 4 \mathrm{PAM}, \approx 336 / \text { symbol in } 8 \mathrm{PAM})\end{array}$ \\
\hline Inner Decoding: & $\begin{array}{l}\approx 3,515,800 \text { computations } \\
\quad\left(2 n \log _{2}(n)+4 n+11 n / k \text { where } n=2^{m}\right. \\
\quad \text { for each } \operatorname{RM}(1, \mathrm{~m}) \text { region })\end{array}$ \\
\hline Outer Decoding: & $\begin{aligned} \approx 53,210,028 \text { computations } \\
\\
\quad\left(I_{\text {avg }} \cdot 63.5 \cdot k\right. \\
\quad \text { where } I_{\text {avg }}=\text { average no. iterations }=18, \\
\quad k=\text { number of info bits }=46,553)\end{aligned}$ \\
\hline
\end{tabular}

* Fast DCT algorithms generally assume an input block of base 2. Specialized algorithms are be required for other input block sizes (such as 400 as indicated above) which reduce efficiency. The number of DCT computations provided above acts as an estimate - no particular fast DCT algorithm has not been specified for the receiver (see Section 5.2.1).

Table 7.2: Software Receiver Complexity for $400 \times 400$ Pixel Barcode 


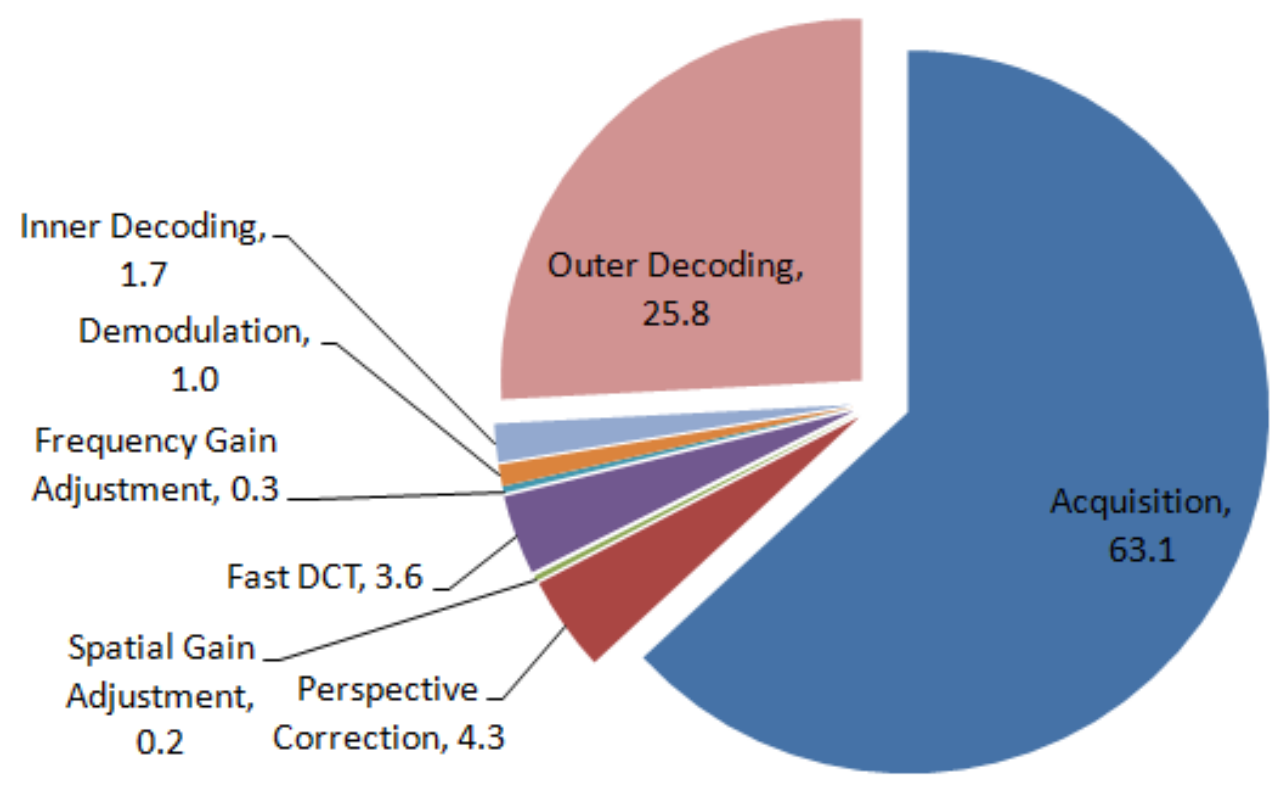

Figure 7.2: Breakdown of Computational Complexity at the Receiver (expressed as percentages of total computation time )

are required in order to acquire the barcode on the first scan of the photograph. Larger fiducials can easily reduce complexity at the receiver, but at the cost of capacity. Future efforts to reduce complexity should focus on the acquisition method, as the size of the fiducials and the number of matched filter operations applied was not optimized mathematically (a more practical approach was taken).

The final barcode was designed to be $400 \times 400$ pixels (square) with fiducials 31 pixels in diameter and a 2-pixel-wide gray border to prevent the photograph background from blurring into the barcode. This design can certainly be extended to different sized barcodes, and the $400 \times 400$ version is simply an example of what can be achieved. The radial frequency regions can be defined by radius as a percentage of the barcode width/height, and the regions will then scale with different sizes.

The code rates discussed in Chapter 6 were chosen such that the barcode would be decodable under the worst channel conditions as discussed in Section 4.7.3. The barcode performed well with high distortion simulation photographs, decoding in under 30 iterations (with an average of 18 iterations). 


\subsection{Tests and Results}

The $400 \times 400$ barcode was compared to the HCCB for an LCD transmitter. The 4-colour HCCB was tested with the simulator and corrected using the perspective correction technique (with a manual acquisition) to ensure a fair comparison.

A $400 \times 4001.306 \mathrm{~KB}$ barcode appeared to have roughly $92 \%$ of all pulses (triangles in Figure 2.5) being distinguishable, provided a good decoding mechanism is available from Microsoft (warping and blurring altered the position and size of the pulses). Therefore, it was estimated that a 1.2KB HCCB 4-colour barcode was comparable to the $5.8 \mathrm{~KB}$ RGB DCT barcode. The 8-colour HCCB was also tested. It was found that the pink and white pulses were not easily distinguishable. Assuming the Microsoft colour-correction pallet (see Section 2.2.4) corrected this problem, it was estimated that a 1.6 KB HCCB 8-colour barcode was comparable to the RGB DCT barcode of $5.8 \mathrm{~KB}$.

Overall, the new RGB DCT barcode has approximately 3.5 times the data density of the Microsoft HCCB - the leading barcode for camera phone applications. This is a good result, though much more modest than the increase in data density given for orthographic projection as presented by Gupta [33]. More regimented field tests will provide a better comparison of the two symbologies. The processing and decoding software for the HCCB was not available, so it is unclear how well it performs in realistic settings. Additionally, only real trials with camera phones will reveal whether the simulator is a good enough model.

It should be noted that the 0.29 bits/pixel density for an LCD transmitter and camera phone receiver cannot be scaled directly to the $b i t s / \mathrm{cm}^{2}$ metric used for the symbology comparison in Table 2.1. The comparisons in Section 2.3 assume a scanning resolution equal to the printing resolution and do not specify the colour quality. For such a metric, it is reasonable to assume that the RGB DCT barcode holds at least the same data-density advantage over the HCCB as in the LCD transmission scenario, and likely a much greater advantage. With ideal colour response and lighting, the density of the RGB DCT barcode is far greater than that of the HCCB (theoretically, with printing and scanning at $600 \mathrm{dpi}$, the RGB DCT barcode presented can encode 0.29 bits/pixel $\cdot\left(600 \times 600\right.$ pixels $/$ inch $\left.^{2}\right)$. $\left.\left(1 \mathrm{inch}^{2} / 6.4516 \mathrm{~cm}^{2}\right)=16,182 \mathrm{bits} / \mathrm{cm}^{2}\right)$. Of course, this is unrealistic for camera phone applications, as the scanning resolution will not be $600 \mathrm{dpi}$, and colour and lighting responses vary immensely.

Although field testing was out of the scope of this thesis, some tests were done with the MOTOROKR phone using an LCD transmitter. The performance was limited, primarily due to lighting effects. While the barcode performed well in good, even lighting, mirroring 
effects on the LCD transmitter, poor lighting, and shadowing were problematic - particularly for the lowest frequency bins. The tests also indicated that due to the LCD brightness, higher performance is achieved when pre-distortion is omitted (further testing with several phones is required for verification).

It was found that the LLR distributions over each radial frequency region were roughly equal for tests with the camera phone, with the exception of regions 1 and 2 (see Figures 6.3 and 6.4 for regional break-down). When the rate of region 2 was reduced from 2.25 to 2 in each colour channel, the performance with the LCD and phone increased significantly. (Rates elsewhere were increased to achieve the same data density.) The results suggest that the relative coding rates and regional differences are well-suited to the channel (with small adjustments made for region 2 ).

Region 1 continued to perform very poorly, even at low-rates, indicating that the current gain control and processing in the spatial domain is inadequate for overcoming shadowing and other poor lighting effects. This must be addressed before a commercial application becomes feasible. Developing a gain control method for the spatial domain could greatly improve performance and allow for a more data-dense design.

An alternative is to leave the lowest frequency bins uncoded, creating an annulus in the DCT domain of encoded bins. The maximum encoding radius can also be extended, employing lower rate Reed-Muller codes, resulting in the same overall rate.

The coarse acquisition method performed well with zero false acquisitions. The current thresholds may need tuning over time, and it is recommended that the software be adaptive.

\subsection{Suggestions for Future Work}

There are many features of the barcode that can be further studied and improved. A more robust fine-acquisition method is recommended, as several failed trials were partly a result of imprecise perspective correction. It would also be of great value to develop a method of equalizing the fiducials at the receiver. From Figures E.1 and E.2 in Appendix E, it is clear that the super-imposed fiducials have a detrimental effect on data density even once they are replaced by the mean received pixel value.

For LCD transmissions, spatial domain processing must account for a variety of lighting effects that were not well modelled in the simulator. Further tests are required to properly model the array of lighting possibilities, and some simple processing steps for the receiver should be sought. If at some point the raw image becomes available to the receiver in the camera phone, processing will be much more straight-forward and a much higher datadensity could become feasible. Strong colour-correction and lighting adjustment techniques 


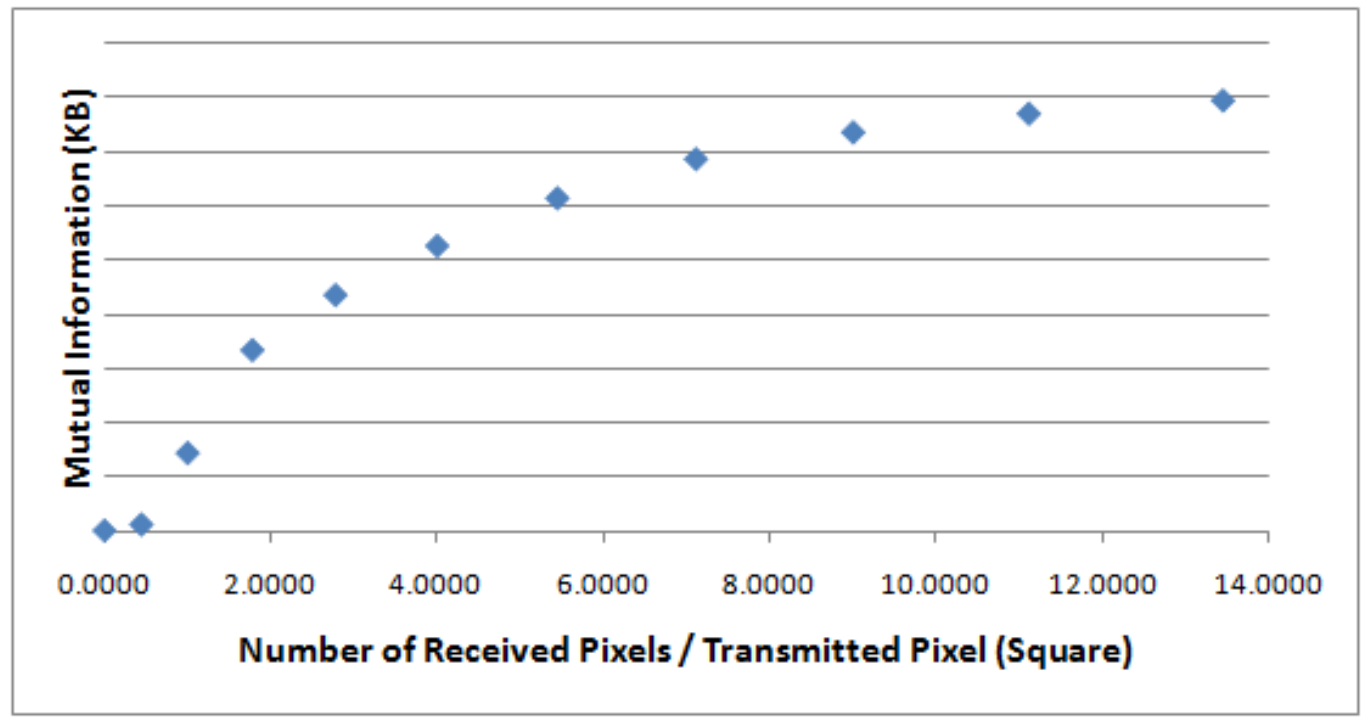

Figure 7.3: Effect the Ratio of Received Pixels to Transmitted Pixels has on Capacity (all other channel parameters held constant)

could be employed, thus eliminating many of the non-linearities in the channel.

Pilot tones were not employed for the DCT RGB barcode, but have the potential to provide a more robust design. Pilot tones in both the spatial and frequency domains could provide the receiver with information allowing for colour and contrast adjustments, more sophisticated gain control, and other channel equalization techniques. It would be worthwhile to test strategies that utilize pilot tones and incorporate a pilot tone scheme into the barcode symbology.

The barcode was not designed with paper transmission in mind, but will likely perform well in such applications. Colour ink may be problematic, depending on the application, thus making a monochrome black and white version appealing. Paper/surface transmissions should be evaluated based on resolution, colour fidelity, lighting, etc. The barcode rate may need to be adjusted accordingly.

It was found that the ratio of the barcode size to photograph size had an immense impact on performance. Holding all other factors constant (lens PSF, CMOS noise, etc.), Figure 7.3 illustrates the effect the number of received pixels per transmitted pixel has on capacity (simulation results).

While increasing the camera resolution may have similar effects, it was seen in Chapter 3.3.1 that the CMOS imager noise increases if the number of pixels increases for a fixed chip size. Photographs in which the barcode makes up the majority of the shot allow for a better use of resources and higher performance. 
One suggestion is to create a black frame in the LCD on the camera phone (through which users view the scene they are photographing), indicating to the user how large the barcode should appear. Figure 7.4 offers an illustration of this concept. This may also reduce the degree of warping. However, it is not clear when the frame should appear, as users will probably not appreciate it for regular non-barcode photographs. More importantly, because there is currently no optical zoom on most camera phones, the frame may require the user to move closer to the barcode which may not be possible or desirable. One particular instance is for LCD transmitters: photographing too closely results in poor performance due to the lighting effects. Overall, the frame may be very valuable for paper transmissions, but for LCD and CRT transmissions, further testing is required.

It was observed that while photographing very close to the LCD created a high barcodeto-photograph ratio, the poor lighting effects generally overwhelmed the gains. The performance for a large screen, such as in a presentation room, may be greater (users will be farther away, but the barcode-to-photograph ratio may remain high). This may be beneficial for presentations, such as PowerPoint, in which information about the presentation/presenter could be encoded in a barcode and displayed at the end for the audience.

Further work may include extending the barcode design to accommodate a variety of sizes. (Note that power of two dimensions enable a more efficient 2D-DCT operation.) The receiver will be required to determine the dimensions at the time of acquisition. Therefore, some spatial domain indicators will be required (perhaps incorporated into the fiducials).

It is hypothesized that real-world trials will yield several areas in need of improvement. At this point, there is no software receiver implementation for mobile phones. While consideration to the processing capabilities of mobile phones was taken in the design of the receiver (processing and decoding), it would be worthwhile to observe whether users are discouraged by the wait time.

In summary, almost every aspect of the design could be further studied and optimized. Several steps were taken based on simulation and experimentation. The water-filling power allocation, for instance, is not mathematically optimal, nor is the perspective correction method and spatial domain gain adjustments, or even the code rates. This first-run design acts more as a good starting point and an indication of what can be achieved if such a product is desired. 

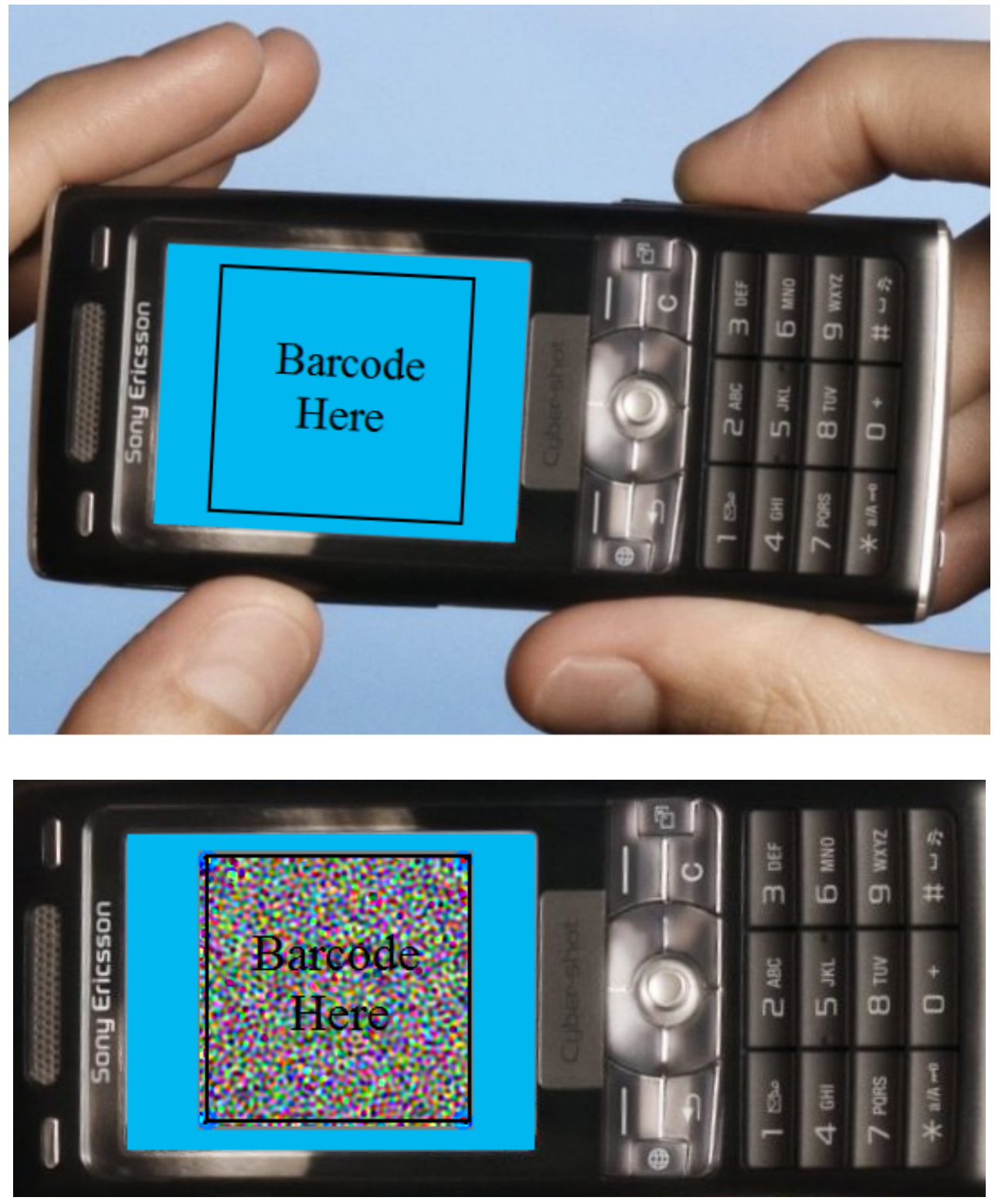

Figure 7.4: Framing where the barcode should appear in photograph on the phone LCD [129] 


\section{Chapter 8}

\section{Conclusion}

A high density barcode to be photographed and decoded by mobile phones has been presented. A channel model was derived and a Matlab channel simulator was developed, leading to a conservative capacity estimate of $6.5 \mathrm{~KB}$ for a $400 \times 400$ pixel barcode with LCD transmission.

A barcode symbology is presented with encoding done in the DCT domain. A waterfilling process allocates power to low frequency bins, and a noise-shaping algorithm is employed to reduce clipping noise. A rotation and size-invariant acquisition method is provided, allowing for faster processing at the receiver.

The final RGB DCT barcode, designed for mobile phones applications, has a data-density of $\approx 0.29$ bits / pixel (for an LCD transmitter). Simulation results indicate that the new symbology has roughly 3.5 times the data density as the 8-colour HCCB (the leading barcode in density for camera phone applications). However, some preliminary test results indicate that more robustness to various lighting effects is required for a commercial application. This may be achieved through spatial domain image processing. Otherwise, the increase in density may be lost due to corruption of the lower frequency bins.

Other transmitters, as well as monochrome versions of the barcode, are worth studying. When the raw image data in camera phones becomes available to the receiver, it is expected that data density may increase immensely due to the subsequent processing possibilities. Given the current camera phone technology, the RGB version may be too sensitive to nonlinearities, particularly those of lighting effects, to maintain a significant advantage over the HCCB in commercial applications. Meanwhile, a black and white monochrome version may be much more robust as colour is more affected than relative luminance by lighting conditions.

Overall, the symbology design reveals that greater data densities can be achieved for 
barcodes by encoding data in the frequency domain. Doing so provides robustness against spatial domain low-pass filtering (blurring) caused by camera lenses and image sensors. Furthermore, the defocus, motion blur, and JPEG compression inherent in mobile phones is less cumbersome to a low-frequency-encoded barcode than the more common square or triangular pulse symbologies. DCT encoding, with code rates varying with spatial frequency, appears to be a very sound approach, and the Reed Muller-ARA code combination works well.

While further work may be required for a commercial application, the current design is a good starting point. Further testing with various camera phones will highlight areas in need of improvement, thus continuing the iterative design process. The channel simulator is a useful tool that can be used for optimization and further development. It is expected that an SDMT approach to barcode symbologies has a lot of potential with camera phone applications, and that the design presented is a good preliminary version. 


\section{Appendix A}

\section{Data-Density Calculations}

\section{INTACTA.CODE}

- 3800 bytes per square inch at $600 \mathrm{dpi}$

$$
3800 \frac{\text { bytes }}{\text { inch }^{2}} \cdot \frac{\left(1 \mathrm{inch}^{2}\right)}{\left(6.4516 \mathrm{~cm}^{2}\right)} \cdot 8 \frac{\text { bits }}{\text { byte }}=4712 \mathrm{bits} / \mathrm{cm}^{2}
$$

- For more information, see [18].

\section{Data Matrix}

- $80 \times 80$ module symbol contains 454 binary symbols (base 256)

- Minimum requirement of a 1-module-wide quiet-zone

- Assume 4 dots / module

$$
\begin{gathered}
2.54 \frac{\mathrm{cm}}{\text { inch }} \cdot \frac{1 \text { inch }}{600 \text { dots }} \cdot 4 \frac{\text { dots }}{\text { module }}=0.016933 \mathrm{~cm} / \text { module } \\
0.0169933 \frac{\mathrm{cm}}{\text { module }} \cdot 2(1 \text { module })=0.0339866 \mathrm{~cm} / \text { dimension } \\
\frac{454(8 \text { bits })^{2}}{80^{2} \text { modules }^{2}} \cdot \frac{1 \text { module }^{2}}{0.0169933^{2} \mathrm{~cm}^{2}}=1965 \mathrm{bits} / \mathrm{cm}^{2} \\
\frac{1965 \text { bits }}{(1 \mathrm{~cm}+0.0339866 \mathrm{~cm})^{2}}=1838 \mathrm{bits} / \mathrm{cm}^{2}
\end{gathered}
$$


${ }^{* * *}$ Note that these calculations correspond to approximately $29 \%$

error correction.

- For more information, see [6, 21, 36].

\section{QR Code}

- $25 \%$ error-correction (level M)

- 4 dots / module (minimum value recommended)

- 2600 bits in $81 \times 81$ module area

- QR symbol requires a four-module-wide margin around the symbol

$$
\begin{gathered}
0.0169933 \mathrm{~cm} / \text { module } \cdot 2(4 \text { modules })=0.1359464{ }^{+} \mathrm{cm} / \text { dimension } \\
\frac{2600 \text { bits }^{2} \text { modules }^{2}}{81^{2}} \cdot \frac{1 \text { module }}{0.0169933^{2} \mathrm{~cm}^{2}}=1372 \mathrm{bits} / \mathrm{cm}^{2}
\end{gathered}
$$

- Taking into consideration the margin, gives

$$
\frac{1372 \text { bits }}{(1 \mathrm{~cm}+0.1359464 \mathrm{~cm})^{2}}=1063 \mathrm{bits} / \mathrm{cm}^{2}
$$

- For more information, see [23].

\section{HCCB}

- 2000 bytes per square inch at 600 dpi using the 8-colour palette

$$
2000 \frac{\text { bytes }}{\text { inch }^{2}} \cdot \frac{1 \text { inch }^{2}}{6.4516 \mathrm{~cm}^{2}} \cdot 8 \frac{\text { bits }}{\text { byte }}=2480 \mathrm{bits} / \mathrm{cm}^{2}
$$

- For more information, see [27]. 


\section{Data Glyphs}

- 600 dpi

- $7 \times 7 \mathrm{~cm}^{2}$ symbol

- 5 pixels/cell (minimum allowable)

- 255 bytes with 64 parity bytes (yields $25.3 \%$ error correction)

- 178.5714 bytes $/ \mathrm{cm}^{2}$ data density (using DataGlyph Capacity Spreadsheet from Park Research [28])

$$
178.57 \frac{\text { bytes }}{\mathrm{cm}^{2}} \cdot 8 \frac{\text { bits }}{\text { byte }}=1428 \mathrm{bits} / \mathrm{cm}^{2}
$$

- For more information, see [28].

It should be noted that an exact quantitative comparison between 2-D barcode symbologies based on data-density may not be accurate. For instance, error-correction percentages cannot necessarily be made constant for all barcode types. Also, as symbols vary in size, relative data-densities can change. For instance, the $\mathrm{QR}$ code requires a 4-module-wide quiet zone around the symbol. As the symbol size increases, this overhead is less significant, thus the overall data-density increases relatively. Other such non-linear factors influencing data-density may skew comparative data-density perception. Therefore, the comparison between barcode data-density should be made in a more general/qualitative manner. Table 1 conveys the general hierarchy of data-density among the five symbologies examined. 


\section{Appendix B}

\section{Motorola Camera Phone Specifications}

The specifications for the Motorola MOTOROKR phone used for testing and simulator adjustments are displayed in Figure B.1. 


\section{A motorola}

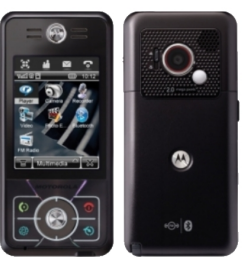

MOTOROKR E6/EGe

\section{Developer Reference Sheet}

\begin{tabular}{|lr}
\hline Technical Specifications \\
\begin{tabular}{lr} 
Band/Frequency & GSM 900/1800/1900 Triband band, GPRS \\
& Class 10, EDGE class 10 \\
\hline Region & EMEA/Asia \\
\hline Technology & GSM \\
\hline Connectivity & EMU (Enhanced Mini USB), Bluetooth, \\
& $3.5 \mathrm{~mm} \mathrm{Stereo/Mic} \mathrm{Jack,} \mathrm{MMC/SD} \mathrm{Memory}$ \\
Card
\end{tabular} \\
\hline Dimensions & $111 \times 51.5 \times 14 \mathrm{~mm} \mathrm{(82 \textrm {cC } )}$ \\
\hline Weight & $122 \mathrm{~g}$ \\
\hline Display & $240 \times 320(260 \mathrm{~K} \mathrm{color})$ \\
\hline Operating System & Linux OS \\
\hline
\end{tabular}

Key Features

Java ${ }^{\mathrm{TM}}$ ME Information
- USB 2.0 Hi-Speed. Bluetooth wireless technology.

- Extended battery life for better usability.

- Supports RealPlayer for music and full screen video and Equalizer in media playe.

- Supports 2 digits FM radio.

- Tactium touch-screen with two position lock key.

- Full SD removable memory card support.

- 2 mega-pixel camera supports video recording and

macro mode for fine shooting.

- Web camera via EMU: works as a web camera via

EMU data cable connected to PC.

- Language packs for user prompts and Voice Recognition.

- Hours of video recording and movie playback

- Video capture/playback/streaming support for

MPEG4 and H.263 formats.

- The Thinnest Entertainment PDA.

CLDC v1.1 and MIDP v2.0 compliant

Heap Size

Maximum record store size (RMS)

MIDlet storage available

Interface connection

Socket, UDP, Serial

Maximum number of Sockets

Image Support

.PNG, .GIF, .BMP, .JPEG, .EMS, .WMP

Double Buffering

Encoding schemes

USASCII, UTF-8 (Unicode), UTF-16 with explicit Byte Order Mark (Part of

IOP), UCS-2

Input methods Touch screen JSRs: 75 PIM, 75 Fileconnection, 82, 118, 120, $\begin{array}{r}135,139,172, \\ 184,185,205\end{array}$

Motorola APIs: Get URL from Flex API

Audio Support $\quad$ AMR-NB, AMR-WB, XMF, WMA v9, WAV, $A A C, A A C+, A A C+$ Enhanced, MP3, RA

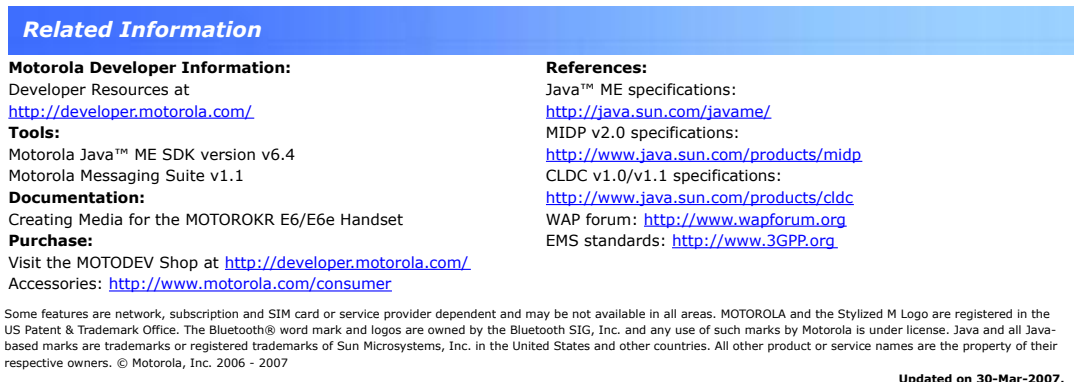

Figure B.1: MOTOROKR E6/E6e Developer Reference Sheet [130] 


\section{Appendix C}

\section{Simulator Settings}

This section aims to illustrate the distortion and noise imposed by the simulator. Figures C.1 to C.4 illustrate the concept of the simulator and how it was used to make mutual information calculations, as well as to test different design features.

While colours in the background of the photograph do have an impact on the final colour response if an automatic white balance (AWB) is used, particularly Gray-World AWB (as in Figure C.3), the photograph backgrounds were not designed to simulate realistic photos

for the simulation trials. The backgrounds were kept white, and AWB was turned off for simulation.

The final simulator settings that were chosen for the mutual information calculations are indicated in Table C.1 and Figures C.6 through C.11 offer some simulated photograph samples with warping for the original transmitted barcode in Figure C.5. 


\begin{tabular}{|c|c|}
\hline Variable Parameters & Range \\
\hline Resolution of Barcode & $400 \times 400$ pixels \\
\hline Physical Size of barcode & assumed to be $4.680^{\prime \prime} \times 4.680^{\prime \prime}$ on LCD \\
\hline Background Illumination & $\{$ dark, very bright, well lit $\}$ \\
\hline Rotation Angle & randomly chosen angle in $\left[0^{\circ}, 5^{\circ}\right)$ \\
\hline Tilt Angle & randomly chosen angle in $\left(-15^{\circ}, 15^{\circ}\right)$ \\
\hline Pan Angle & randomly chosen angle in $\left(-15^{\circ}, 15^{\circ}\right)$ \\
\hline $\begin{array}{l}\text { Size of Barcode vs. } \\
\text { Total Photograph }(\%)\end{array}$ & $30 \%$ \\
\hline Degree of Defocus & $\sigma^{2}=0.7$ \\
\hline Relative Motion & $\approx 9$ received pixels in randomly chosen direction \\
\hline PRNU variance $(R, G$, and $B)$ & $\sigma^{2}=0.0004$ \\
\hline Dynamic Range & {$[0,200]$} \\
\hline Bayer Filter Sampling & standard GRBG Bayer pattern \\
\hline \multirow{3}{*}{ Pixel Cross-Talk } & $\%$ Red leak in RGB = $(92,10,1)$ \\
\hline & $\%$ Green leak in $\mathrm{RGB}=(7,67,18)$ \\
\hline & $\%$ Blue eakin RGB $=(2,21,71)$ \\
\hline Camera Resolution (pixels) & $1600 \times 1200$ \\
\hline Gamma & $\gamma=1 / 2.2$ \\
\hline \multicolumn{2}{|l|}{ Other: } \\
\hline Colour-Correction & None \\
\hline Contrast Adjustments & None \\
\hline AWGN Variance & $\sigma^{2}=0.0001$ \\
\hline JPEG Quantization Tables & $90 \%$ quality \\
\hline
\end{tabular}

Table C.1: Simulator Settings for Mutual Information Calculations 


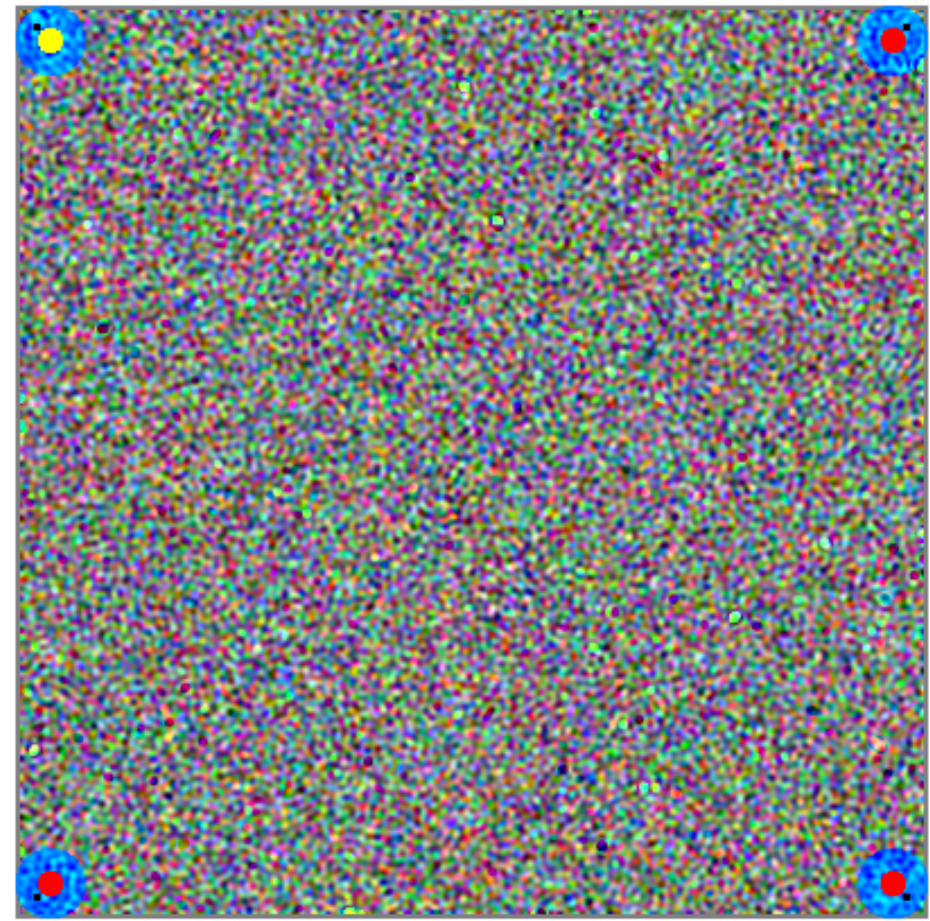

Figure C.1: Original Barcode Transmitted (see Figure C.2) 


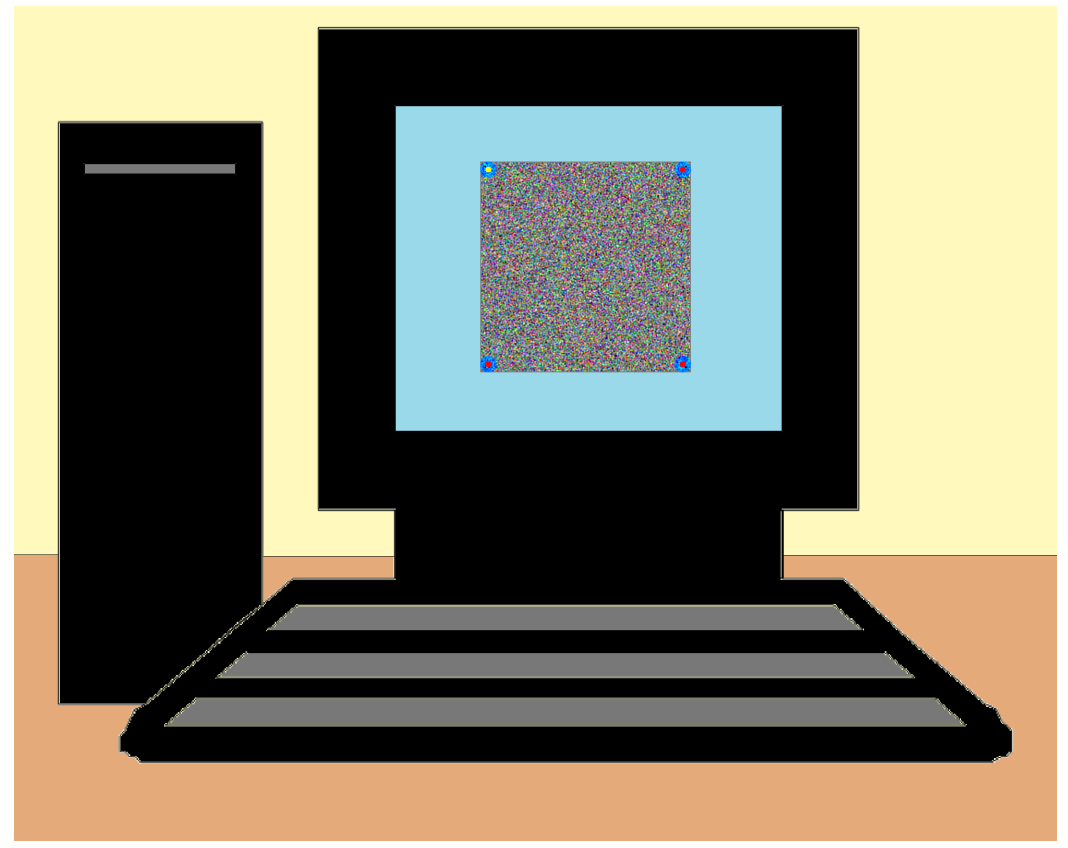

Figure C.2: Simulated Scenario for Barcode Display

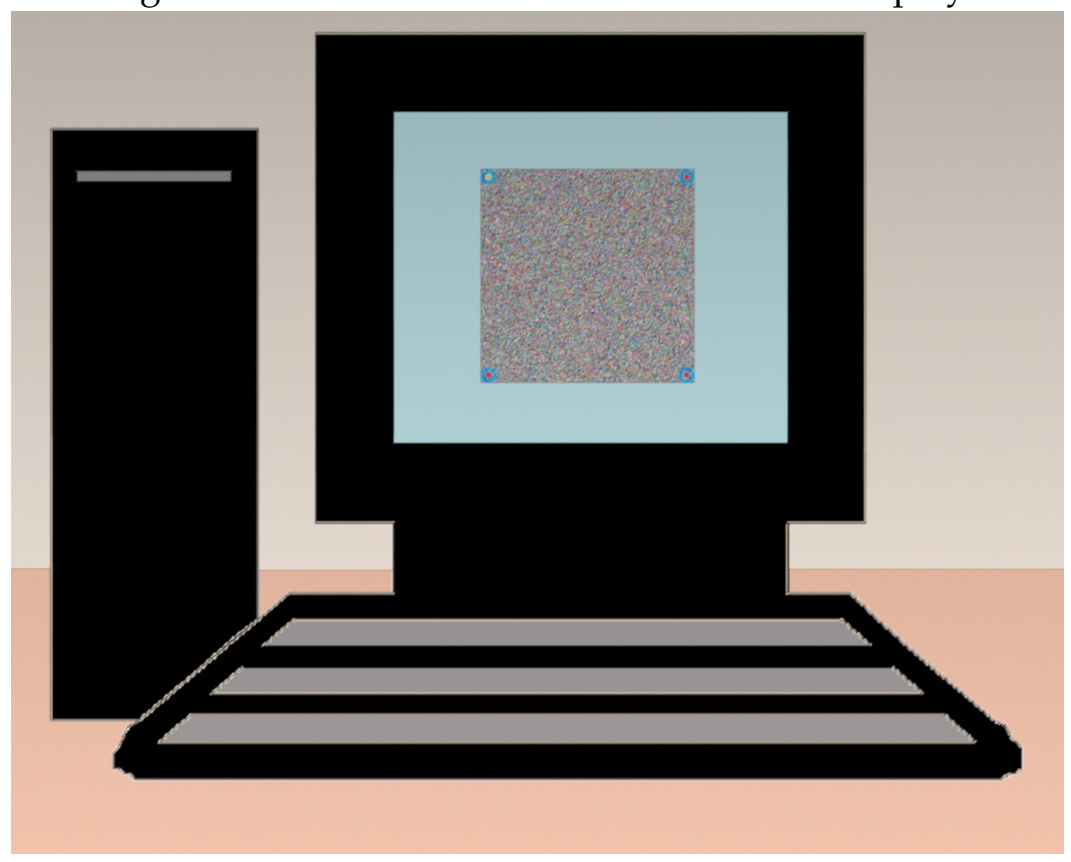

Figure C.3: Simulated Photograph of Barcode (with Gray-World AWB) 


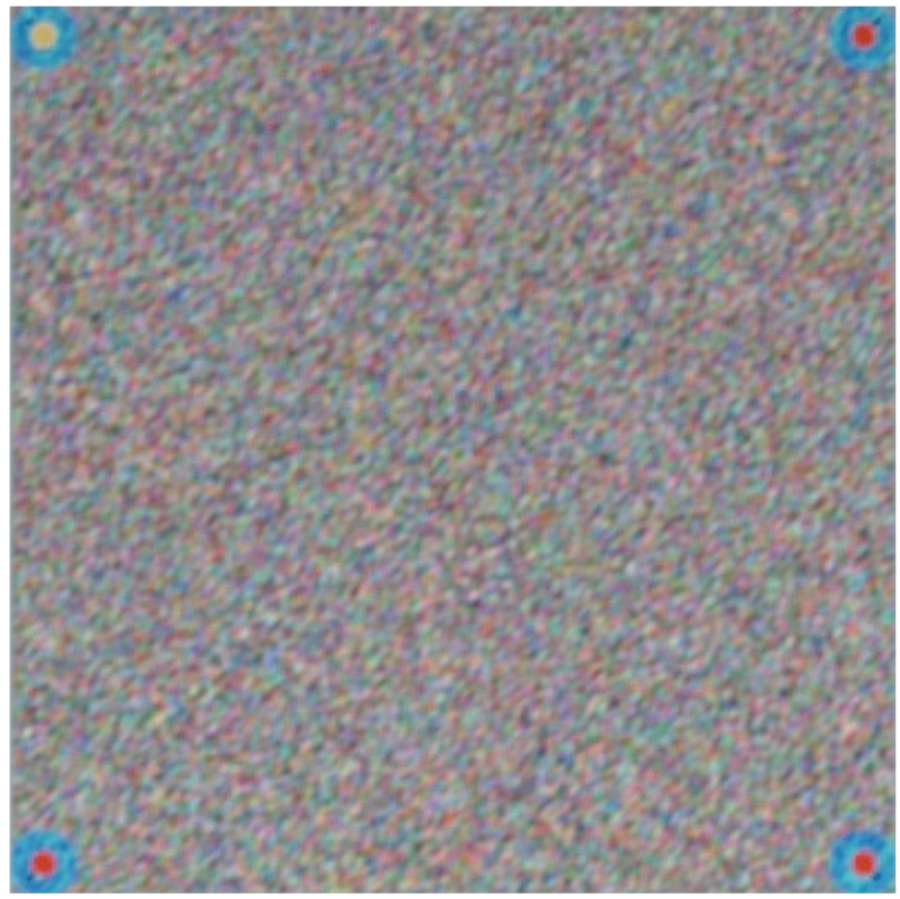

Figure C.4: Resultant Acquired Perspective-Corrected Barcode used to Analyze Capacity and Design Features 


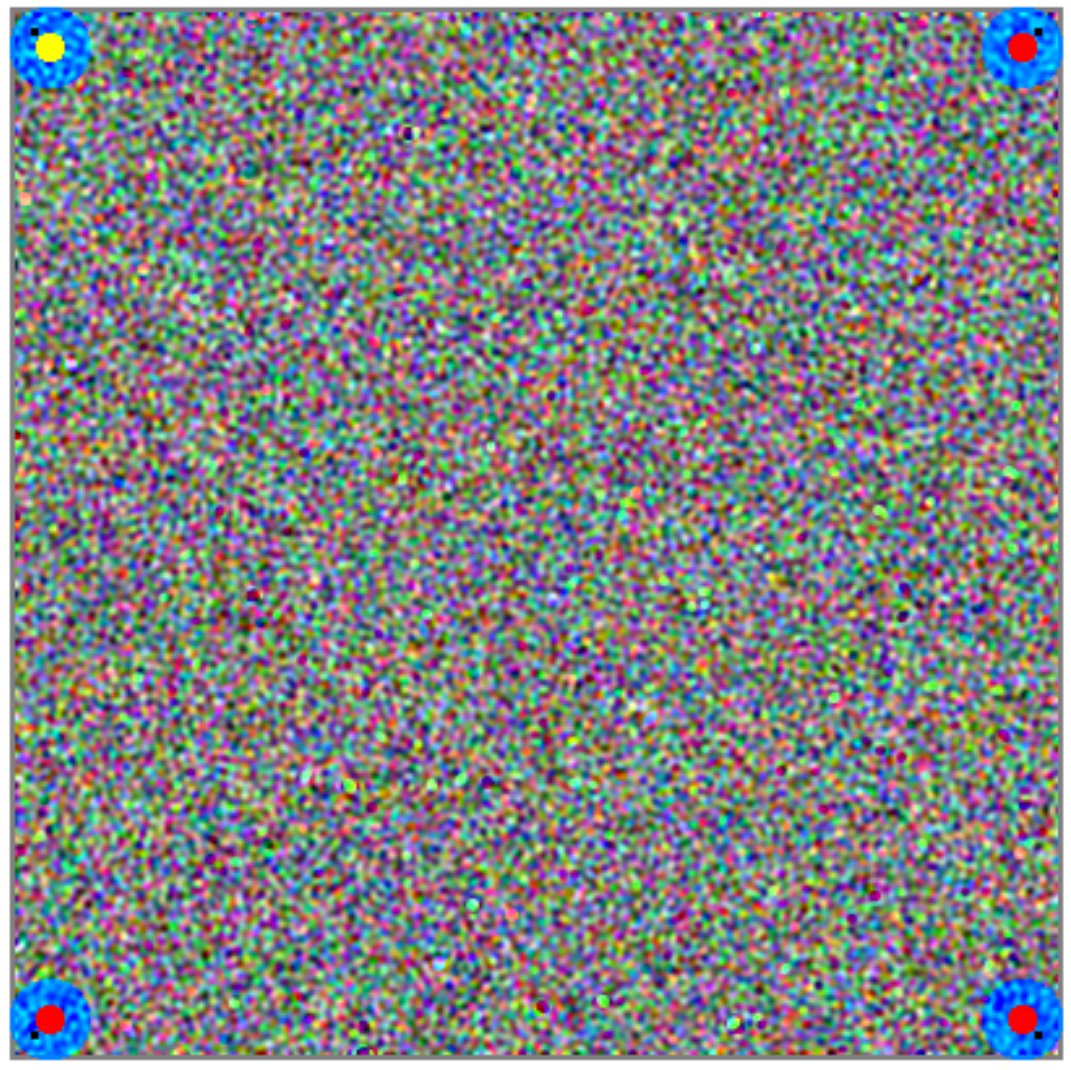

Figure C.5: Original Transmitted Barcode (see Figures C.6 - C.11) 


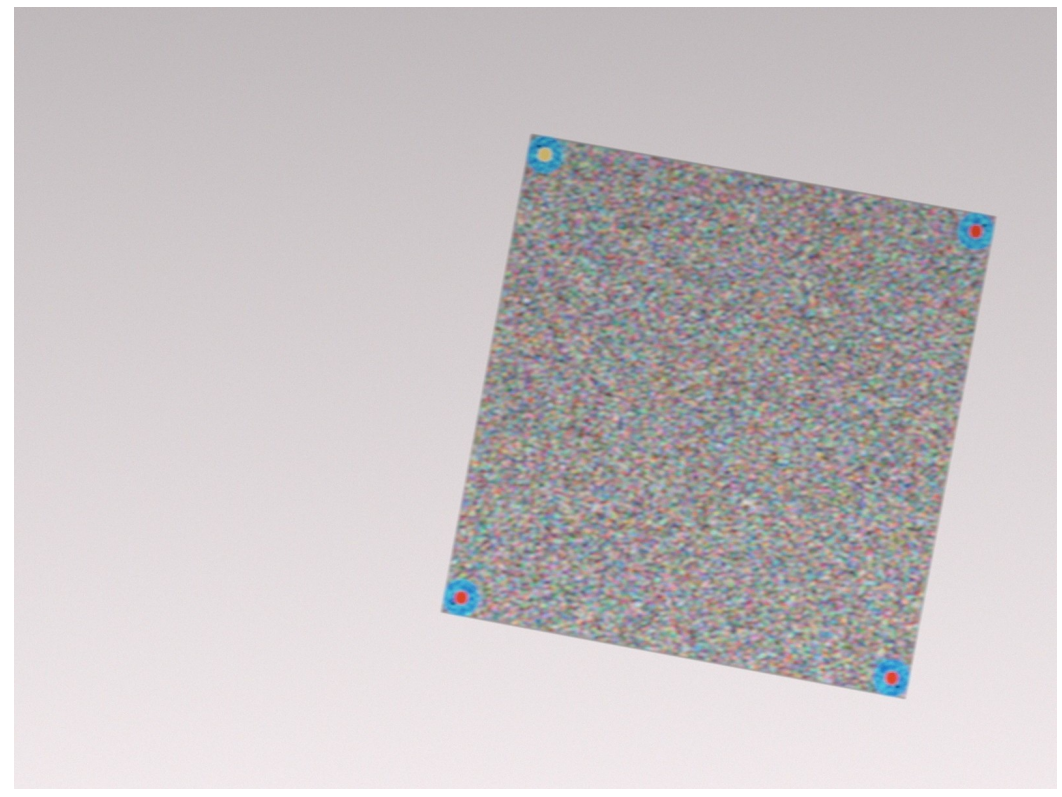

Figure C.6: Simulated Photograph of Barcode

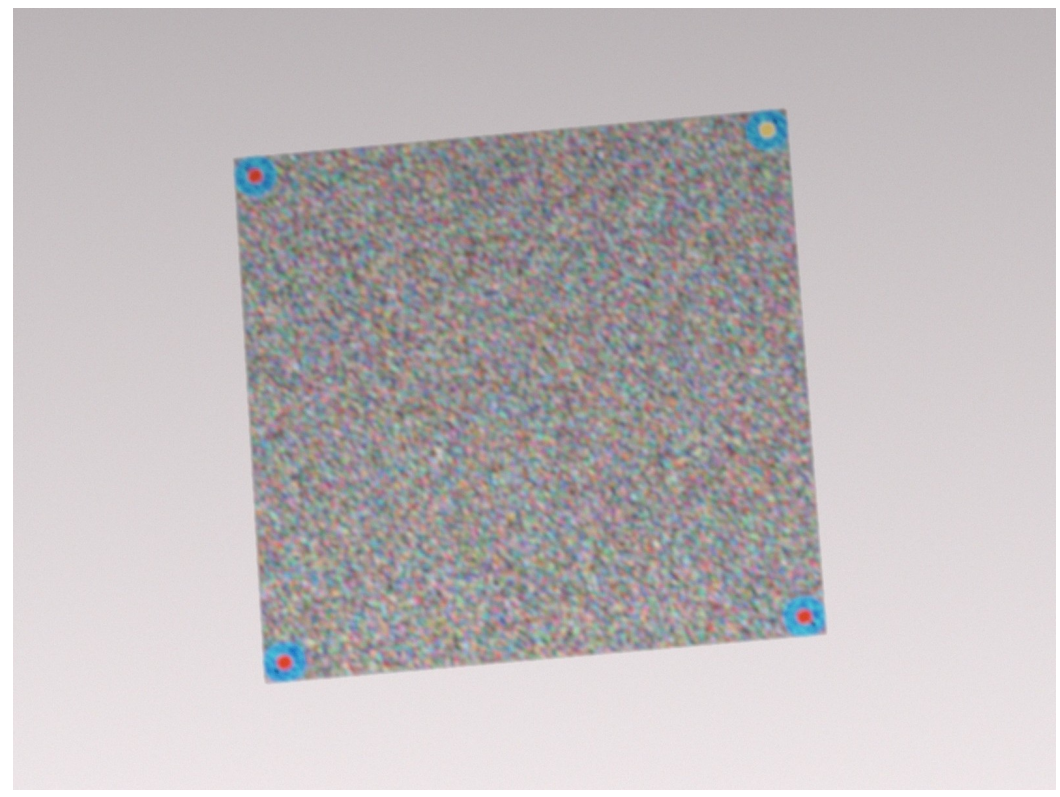

Figure C.7: Simulated Photograph of Barcode 


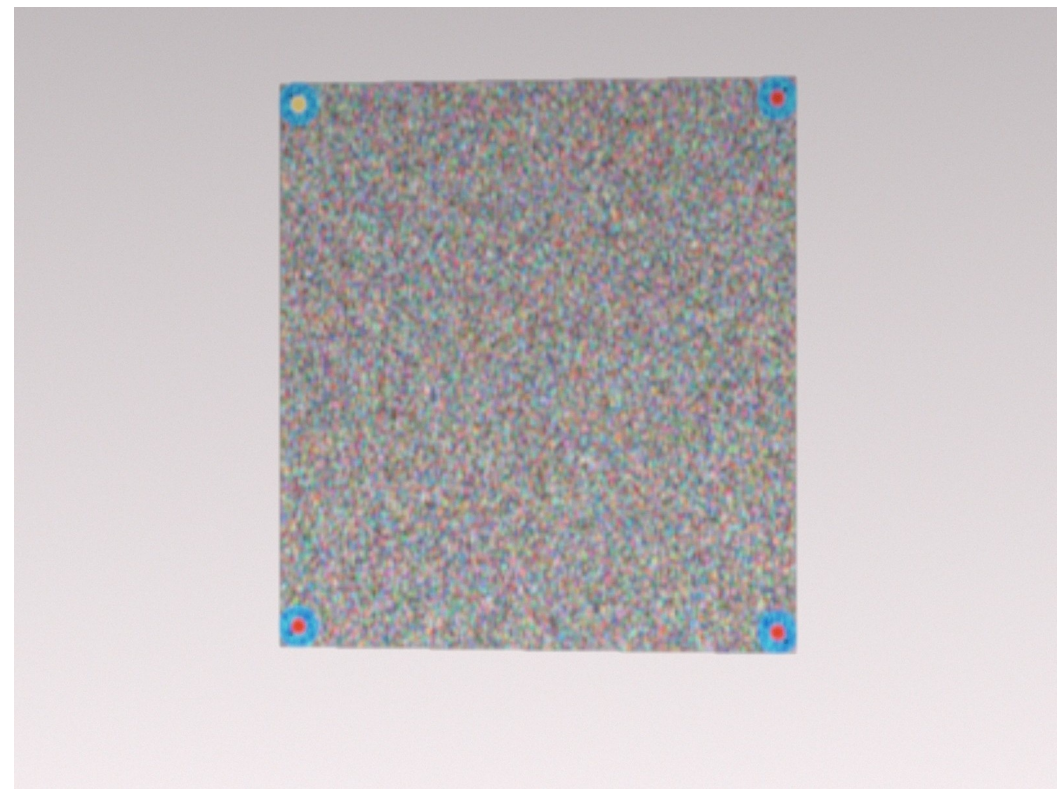

Figure C.8: Simulated Photograph of Barcode

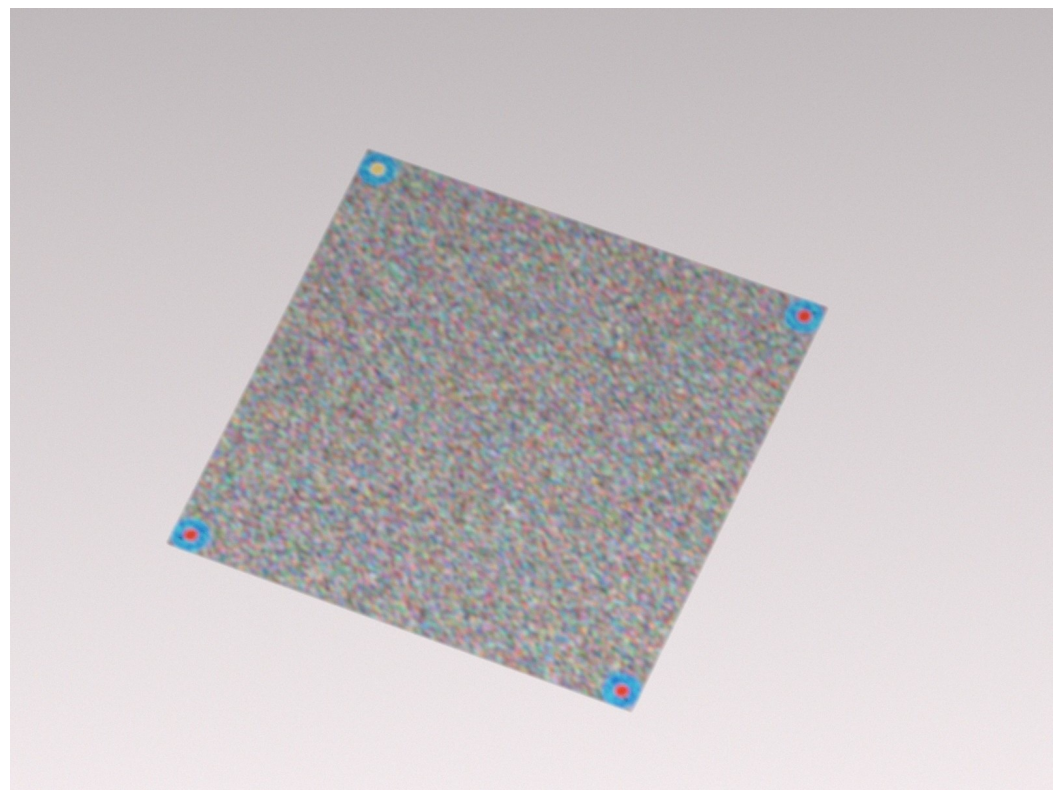

Figure C.9: Simulated Photograph of Barcode 


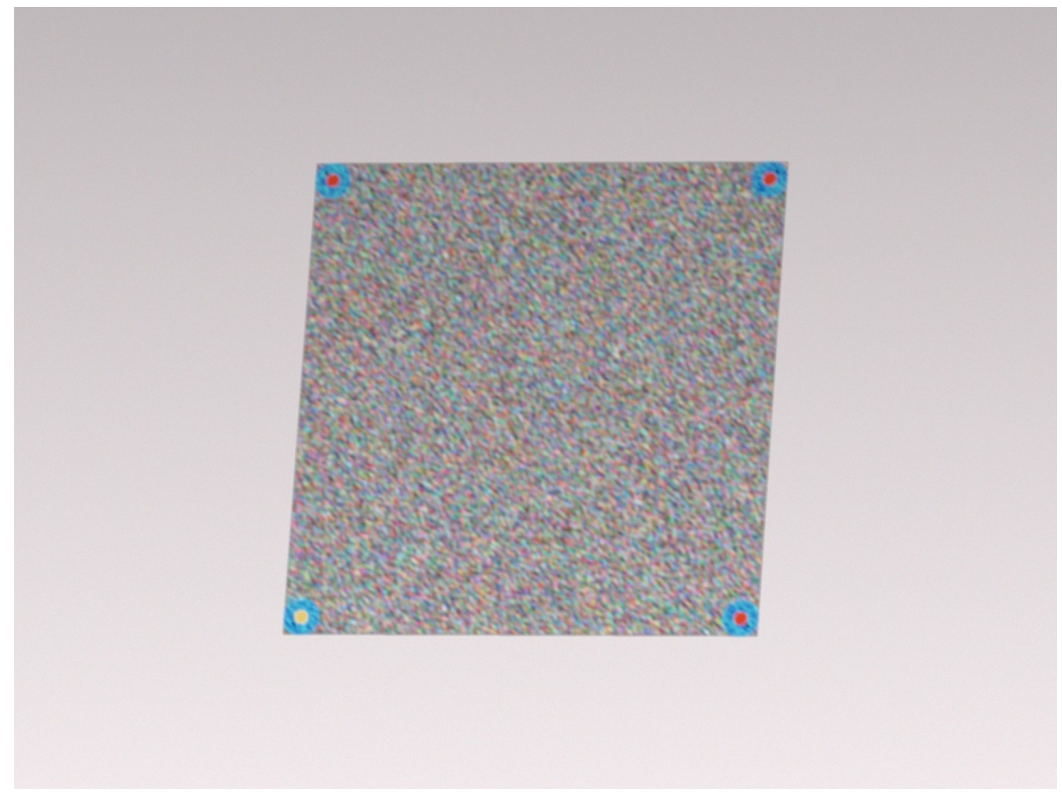

Figure C.10: Simulated Photograph of Barcode

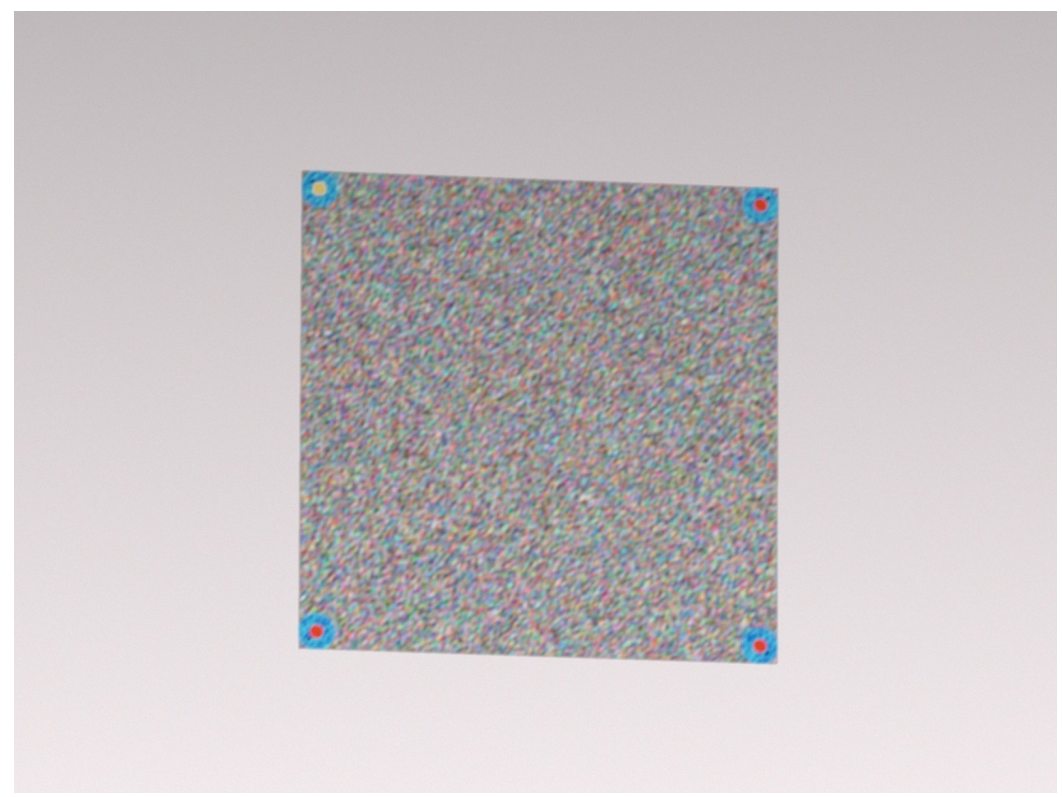

Figure C.11: Simulated Photograph of Barcode 


\section{Appendix D}

\section{Processing}

The sequence of Figures D.1, D.2, D.3, D.4, D.5, D.6 show the effects of processing at the receiver for a successfully decoded barcode sample. 


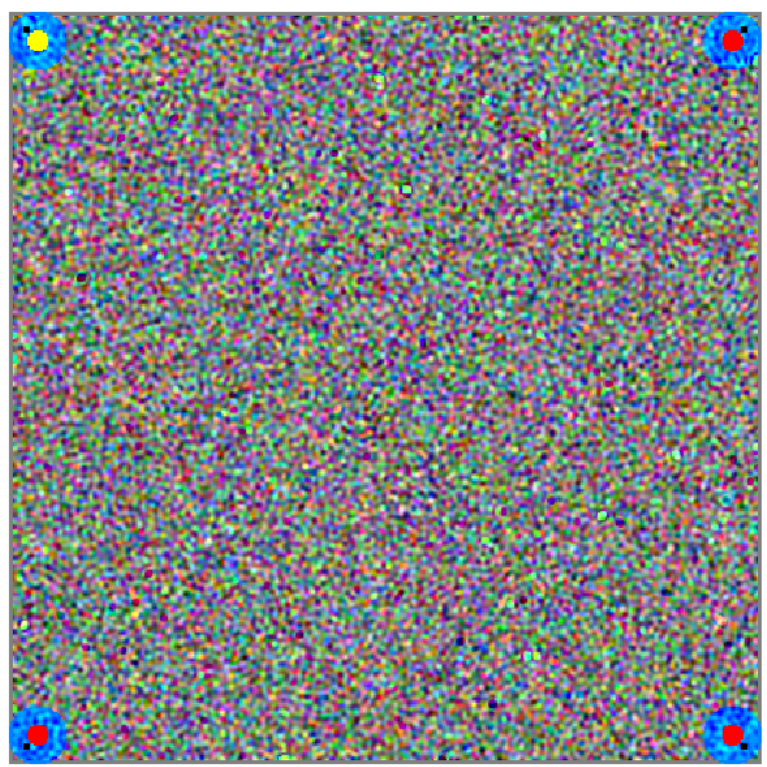

Figure D.1: Original Barcode Transmitted (see Figure D.2)

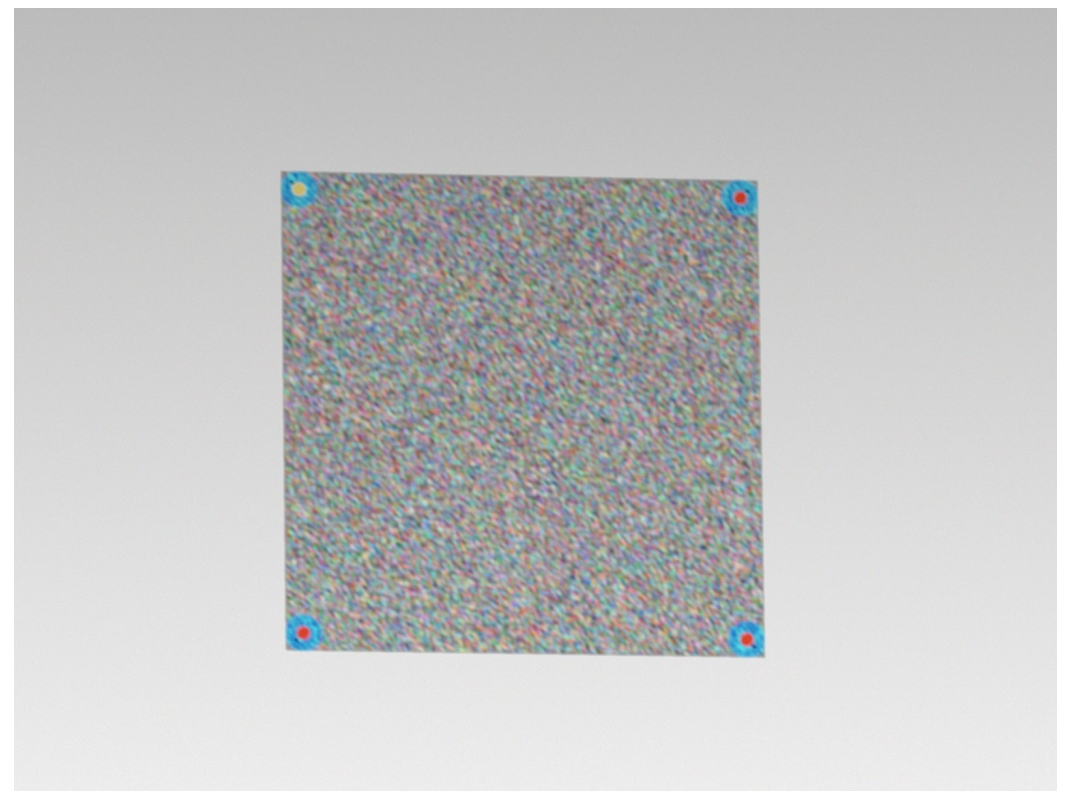

Figure D.2: Simulated Photograph of Barcode 


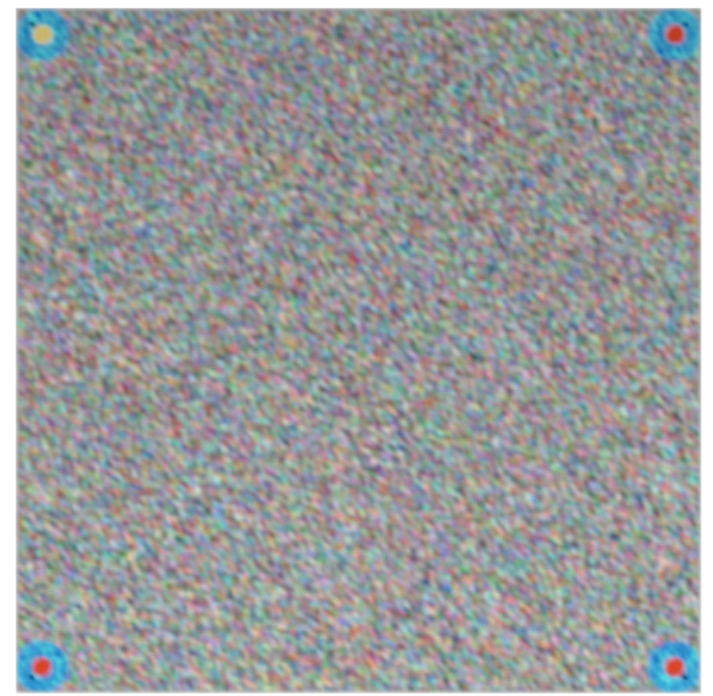

Figure D.3: Acquired Barcode after Perspective Correction

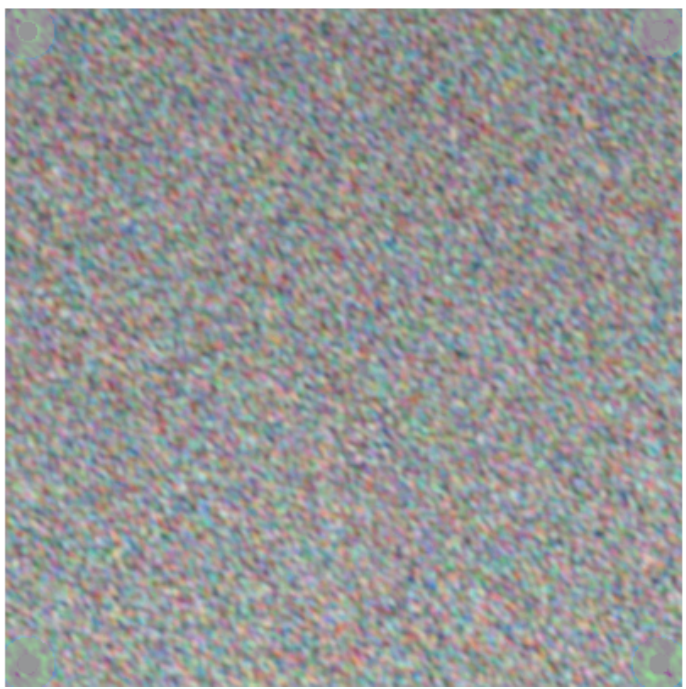

Figure D.4: Received image with fiducials replaced with mean received pixel value in red and blue channels 


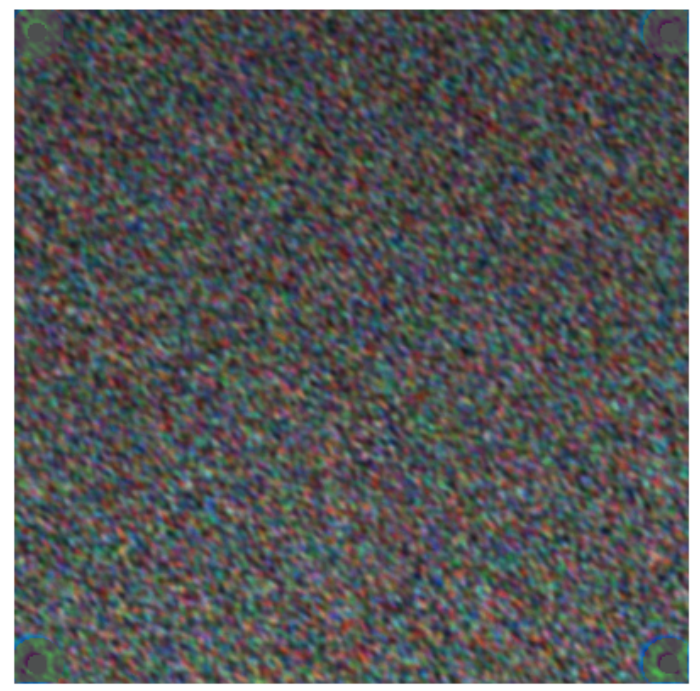

Figure D.5: Received Image after Gamma Curve with Coefficient of 1/2.2 Applied (not normally done - optional)

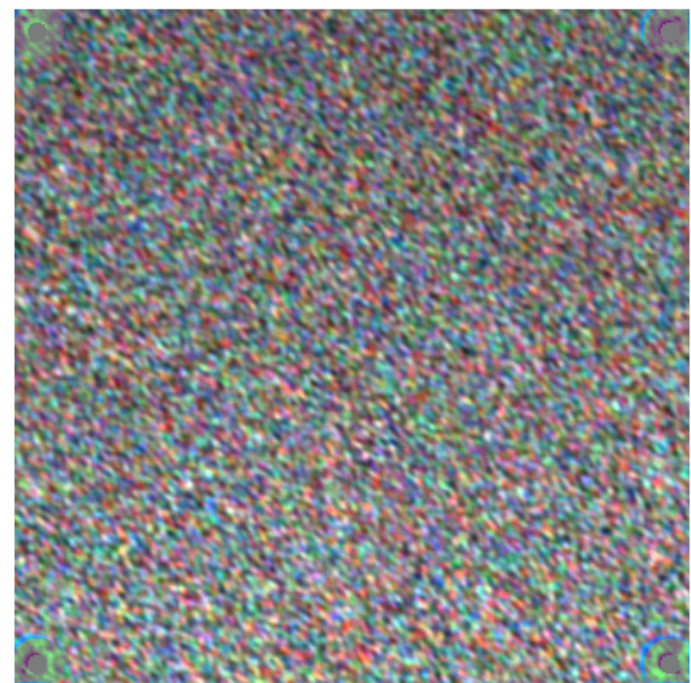

Figure D.6: Received Image After Global Spatial Domain Gain Adjustment 


\section{Appendix E}

\section{Capacity Estimates}

Figures E.1 and E.2 represent the untruncated capacity estimates over the DCT domain for a $400 \times 400$ pixel barcode with maximum encoding radius $=210$. The first image represents the estimated capacity when 31 pixel diameter fiducials are superimposed on the barcode, and the second represents the estimated capacity when the fiducials are kept outside the barcode border. A 2-pixel-wide gray border was included for both cases.

It can be seen that the fiducials have an impact on capacity. The overall estimated capacity of the barcode is reduced by $10 \%$ when 31-pixel diameter fiducials are super-imposed on the barcode versus when they are kept outside barcode borders (assuming that the size of the acquired barcode is equal for both cases). Smaller fiducials have a lesser impact on mutual information, but the acquisition speed and performance suffers.

The measured noise power distributions in each bin were zero-mean and had a decay close to that of a Gaussian distribution (see Figure 5.6). Some bins resembled a Gaussian distribution more closely than others, but for simplicity, the noise was assumed to be additive white Gaussian (AWGN). Figure E.3 illustrates the noise over the DCT domain for the red, green, and blue channels, clearly demonstrating the increase in noise with respect to distance from DC. 


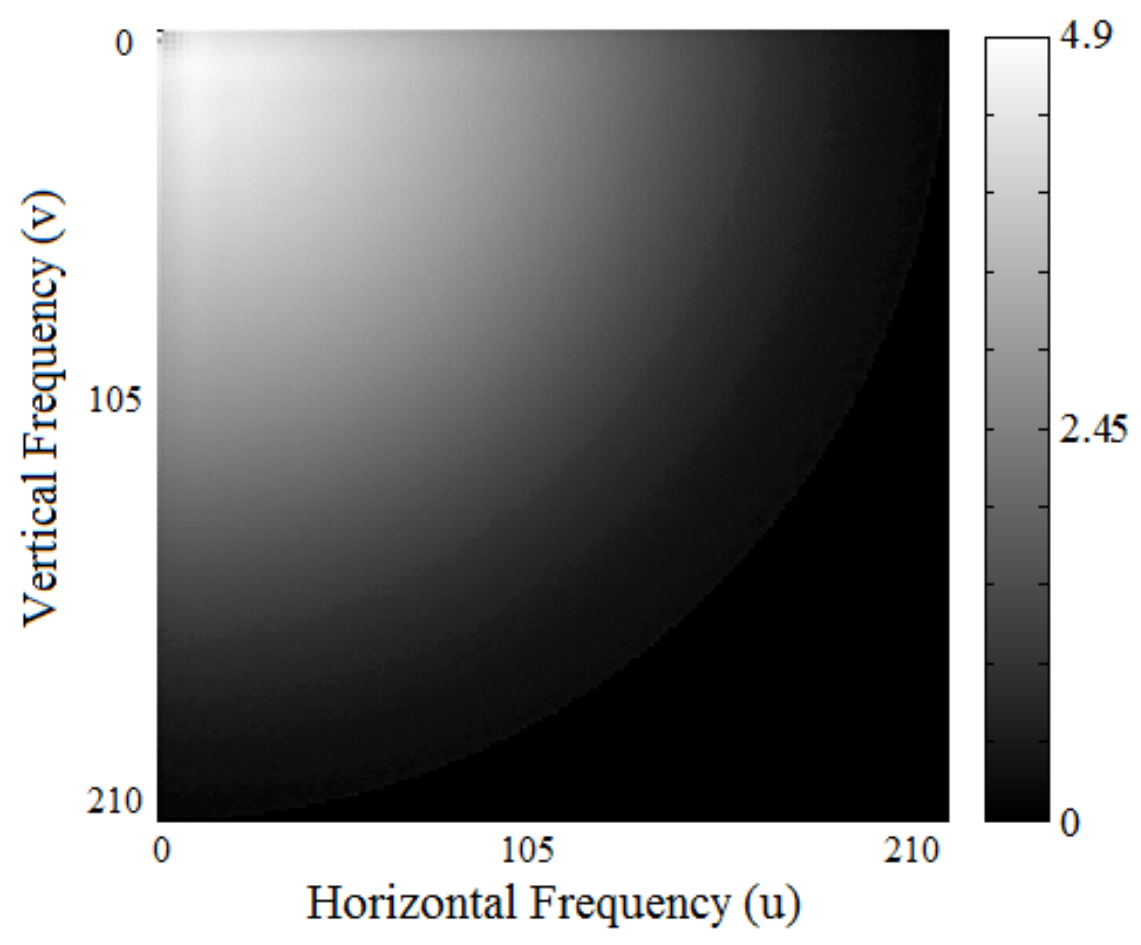

Figure E.1: Image Representation of Estimated Capacity in DCT Domain, 31 pixel diameter Fiducials 


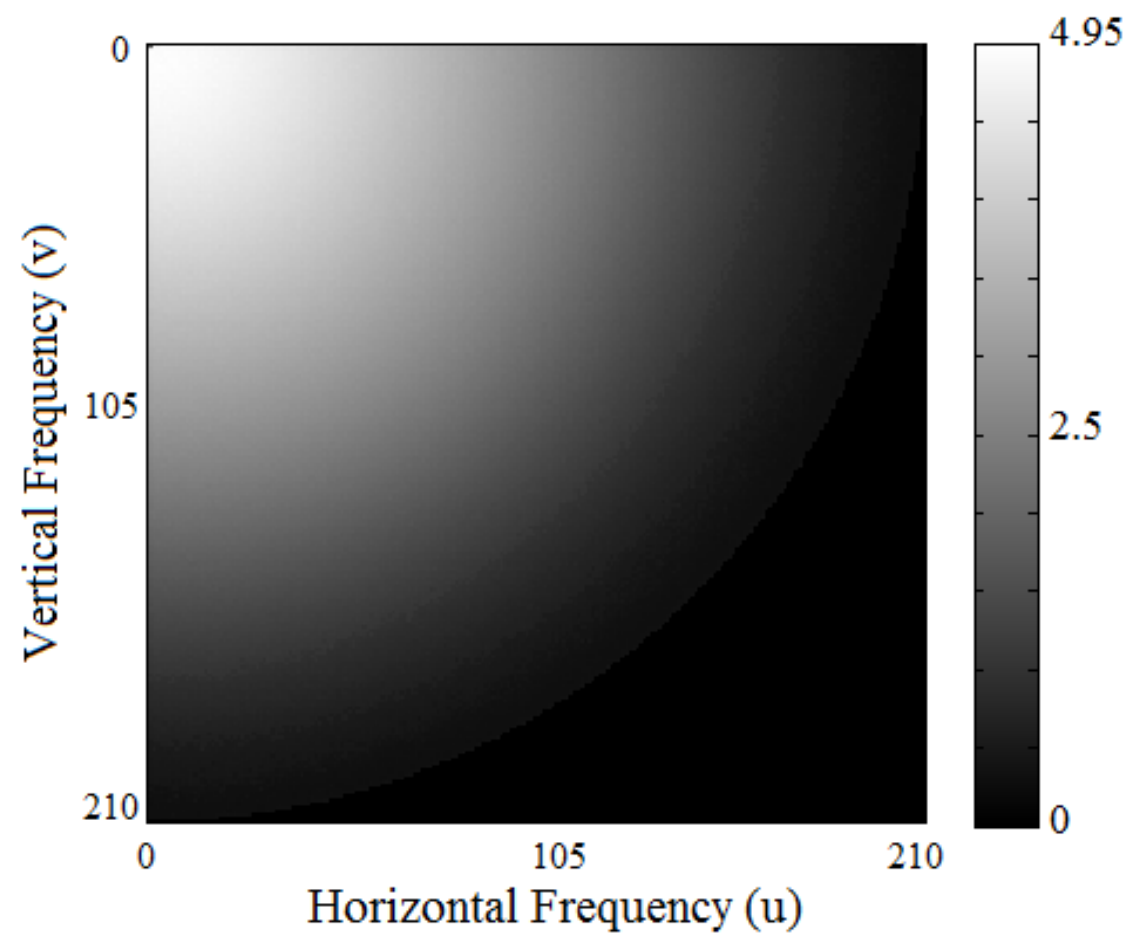

Figure E.2: Image Representation of Estimated Capacity in DCT Domain, No Fiducials (ratio of barcode to photograph kept constant) 

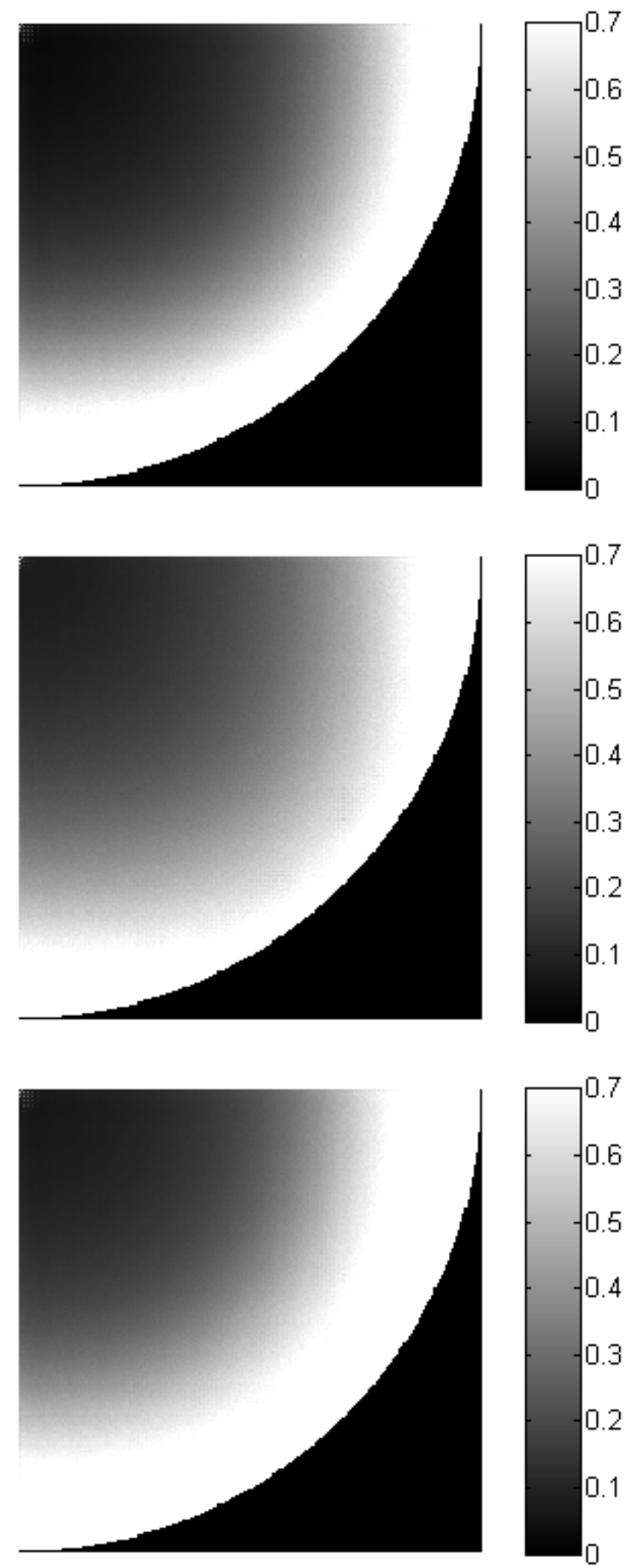

Figure E.3: Noise Power in DCT Domain (From Top: R, G, B Channels) 


\section{Appendix F}

\section{MAP Check Sum Approximation}

In order to reduce the complexity in the belief propagation outer decoding, an approximation for the check sum function is made. Figures F.1 and F.2 illustrate the difference between the true check-sum function (Equation 6.19) and the approximation of Equation 6.20. 


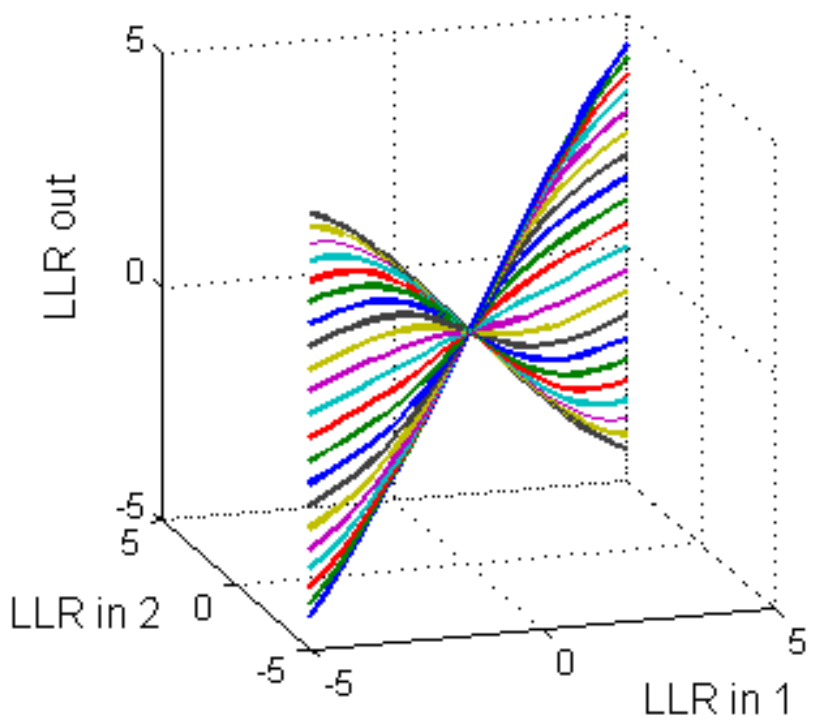

Figure F.1: True Check Sum Function

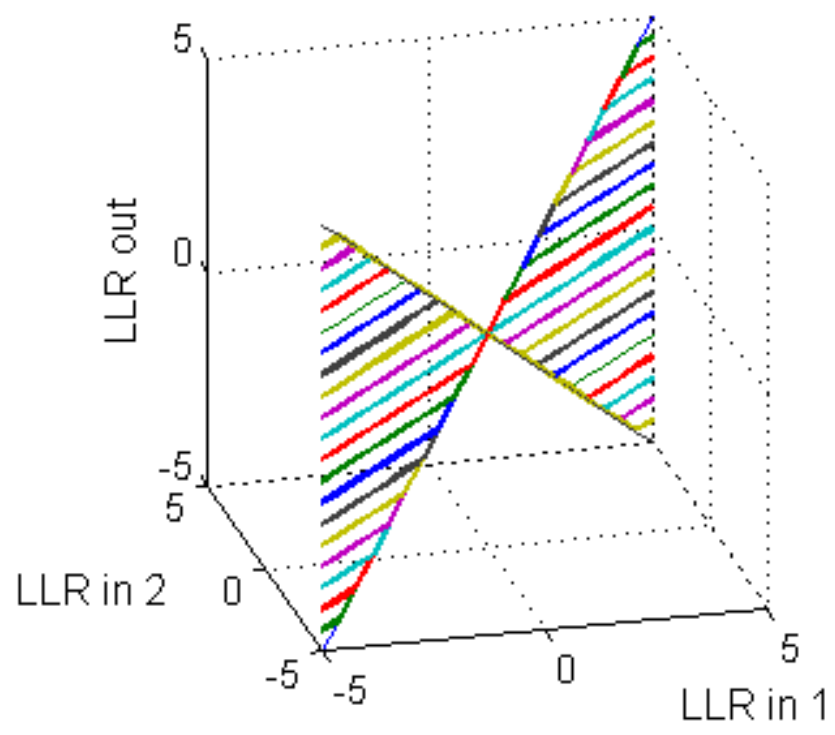

Figure F.2: Check Sum Approximation $L L R_{\text {sum }}=\max \left(L L R_{\text {in } 1}, L L R_{\text {in } 2}\right)-\max \left(0, L L R_{i n 1}+L L R_{i n 2}\right)$ 


\section{Bibliography}

[1] R. Adams. Bar Code 1: 2 Dimensional bar code page. http://www . adams 1.com/ pub/russadam/stack. html, 1995. Accessed 19 February 2008 [Online].

[2] T L Ashford \& Associates. Bar Code Basics. http://www.tlashford.com/ frames/sym_code4 9/sym_code 49.htm, 2007. Accessed 1 March 2008 [Online].

[3] TekLynx Software Solutions. Code 16K. http://www.teklynx.com/barcodes/ article_46.html, 2008. Accessed 1 March 2008 [Online].

[4] Wikipedia the free encyclopedia. Universal Product Code. http://en. wikipedia.org/wiki/Universal_Product_Code, 2008. Accessed 1 March 2008 [Online].

[5] Rosistem Bar Code. Barcode Education. http://barcode.ro/tutorials/ barcodes/index. html, 2003-2008. Accessed 19 February 2008 [Online].

[6] BBC NEWS. Colour barcode system to hit DVDs. http://news.bbc.co.uk/2/ hi/technology/6570871. stm, 2007. Accessed 30 January 2008 [Online].

[7] E. Ohbuchi, H. Hanaizumi, and L.A. Hock. Barcode Readers using the Camera Device in Mobile Phones. In 2004 International Conference on Cyberworlds, pages 260-265, November 2004.

[8] K. Kincade. Barcode scanners aren't just for groceries anymore. Laser Focus World, 41(7):69-73, July 2005.

[9] Current Directions Inc. Sizing Applications for 2D Symbols. http://www. currentdirections.com/facts/ sizing-applications-for-2d-symbols.html\#sizematrix, 2007. Accessed 28 February 2008 [Online].

[10] Fujitsu Ltd. Steganography - Code Recognition Technology. http: //jp.fujitsu.com/group/labs/downloads/en/business/activities/ 
activities-4/fujitsu-labs-imagevoice-003-en.pdf, 2005. Accessed 28 February 2008 [Online].

[11] C.H. Chu, D.N. Yang, and M.S. Chen. Image Stabilization for 2D Barcode in Handheld Devices. In ACM Digital Library, pages 697-706. 2007 International Conference on Multimedia, September 2007.

[12] S.A. Brown. History of the bar code. http://eh. net/encyclopedia/article/ brown. bar_code, August 2001. Accessed 21 January 2008 [Online].

[13] S.A. Brown. Inventors: Bar Codes. http://inventors.about.com/library/ inventors/blbar_code.htm, 2008. Accessed 1 March 2008 [Online].

[14] Neodynamic S.R.L. ISBN Barcode. http: / / www. neodynamic.com/barcodes / ISBN_Barcode.aspx, 2003-2008. Accessed 20 February 2008 [Online].

[15] SageData Solutions. What is a Bar Code? http://whatisabarcode.com/, 1991. Accessed 3 March 2008 [Online].

[16] OCR Ltd. Bar Code Technology. http://www.ocr. ca/barcode/barcode.asp, 2008. Accessed 17 February 2008 [Online].

[17] Office of the Privacy Commissioner of Canada. RFID Technology. http://www . privcom.gc.ca/fs-fi/02_05_d_28_e.asp, 2006. Accessed 17 February 2008 [Online].

[18] D.C. Allais. Multitrack Bar Code and Associated Decoding Method. U.S. Patent 4,794,239, Intermec Corporation, December 1988.

[19] Neodynamic S.R.L. Code 16K Barcode. http://www.neodynamic.com/ barcodes/Code_16K_Barcode.aspx, 2003-2008. Accessed 20 February 2008 [Online].

[20] Intacta Technologies Inc. INTACTA.CODE Technology Explained. http://www . intacta.com/InTACTA_CODE/intacta_code.html, 2001. Accessed 5 February 2008 [Online].

[21] M. Tassa, A. Vorboyoff, and G. Naaman. Process for Transmitting, Receiving and/or Storing Information. Canadian Patent Application 2,329,179, Intacta Labs Ltd., October 1999.

[22] T. Nakazawa, K. Hamakawa, Y. Takei, et al. Reading Method of the Two-Dimensional Bar Code. U.S. Patent 7,143,948, Sanyo Electric Co. Ltd., December 2006. 
[23] Wikipedia the free encyclopedia. Data Matrix. http://en.wikipedia.org/ wiki/Data_Matrix, 2008. Accessed 1 March 2008 [Online].

[24] IDAutomation.com Inc. Data Matrix Barcode FAQ and Tutorial. http://www . idaut omation. com/datamatrixfaq. html, 2000-2007. Accessed 5 February 2008 [Online].

[25] D.G. Priddy and R.S. Cymbalski. Dynamically Variable Machine Readable Binary Code and Method for Reading and Producing Thereof. U.S. Patent 5,124,536, International Data Matrix Inc., June 1992.

[26] Wikipedia the free encyclopedia. QR Code. http://en.wikipedia.org/wiki/ QR_Code, 2008. Accessed 1 March 2008 [Online].

[27] Denso Wave Inc. About QR Code. http://www.denso-wave.com/qrcode/ aboutqr-e.html, 2000-2003. Accessed 5 February 2008 [Online].

[28] M. Hara, M. Watabe, T. Nojiri, et al. Optically Readable Two-Dimensional Code and Method and Apparatus Using the Same. U.S. Patent 5,726,435, Nippondenso Co. Ltd. and Kabushiki Kaisha Toyota Chuo Kenkyusho, March 1998.

[29] Microsoft Corporation Research. High Capacity Color Barcode. http: / / research . microsoft. com/research/hccb/about.aspx, 2008. Accessed 5 February 2008 [Online].

[30] Microsoft Corporation PressPass. International Organization Licenses Microsoft's New Multicolor Bar Code Technology for Identifying Audiovisual Works. http: //www.microsoft.com/Presspass/press/2007/apr07/ 0 4-16MSBarCodePR.mspx, 2007. Accessed 1 February 2008 [Online].

[31] G. Jancke. System and method for encoding high density geometric symbol set. European Patent Application EP 1612724 A1, Microsoft Corporation, January 2006.

[32] Palo Alto Research Center. DataGlyphs: Embedding Digital Data. http://www . parc.com/research/projects/dataglyphs/, 2002-2007. Accessed 5 February 2008 [Online].

[33] S. Gupta. Granite Codes: A New Two Dimensional Barcode Symbology. M.A.Sc., University of Toronto, Department of Electrical and Computer Engineering, 2006.

[34] M.D.A. Mohamed and S. Hranilovic. Two-dimensional binary halftoned optical intensity channels. IET Communications, 2:11-17, January 2008. 
[35] SECURITY2image Bulgaria Ltd. S2i matrix codes with high data density. http: // security2image. com/techno. php, 2008. Accessed 25 February 2008 [Online].

[36] Y.H. Liang, Z.Y. Wang, X.Y. Cao, X.W. Xu. Real Time Recognition of 2D Bar Codes in Complex Image Conditions. Proceedings of the Sixth Internation Conference on Machine Learning and Cybernetics, 3:1699-1704, August 2007.

[37] J. Bloomenthal and J. Rokne. Homogeneous Coordinates. http://www. unchainedgeometry.com/jbloom/pdf/homog-coords.pdf. Accessed 19 August 2009 [Online].

[38] I.T. Young, J.J. Gerbrands, and L.J. van Vliet. Image Processing Fundamentals Tutorial. Penhall Publishers website, 2008. http: / /www. imageprocessingplace.com.

[39] I. Carlbom and J. Paciorek. Planar Geometric Projections and Viewing Transformations. Computing Surveys, 10(4):465-502, December 1978.

[40] G. Barbastathis. MIT Open Courseware: 2.71 Optics Course Notes. course website, 2004. http://ocw.mit.edu/OcwWeb/Meachanical-Engineering/ 2-71Fall-2004/LectureNotes/index.htm.

[41] Wikipedia. Fresnel diffraction. http://en.wikipedia.org/wiki/Fresnel_ diffraction, 2006. Accessed 1 August 2008 [Online].

[42] J. Goodman. Introduction to Fourier Optics. Roberts \& Company Publishers, 2005.

[43] Wikibooks, the open-content textbooks collection. Adventist Youth Honors Answer Book/Health and Science/Optics. http://en.wikibooks.org/wiki/ Adventist_Youth_Honors_Answer_Book/Health_and_Science/Optics, 2008. Accessed 5 June 2009 [Online].

[44] S.H. Lee. Optical Information Processing: Fundamentals (Topics in Applied Physics, volume Chapter 1: Basic Principles. Springer Berlin / Heidelberg, 1981.

[45] S.K. Nayar and Y. Nakagawa. Shape from Focus. IEEE Transaction on Pattern Analysis and Machine Intelligence, 16(8):824-831, August 1994.

[46] U. Cilingiroglu, S. Chen, and E. Cilingiroglu. Range Sensing With a Scheimpflug Camera and a CMOS Sensor/Processor Chip. IEEE Sensors Journal, 2004.

[47] C.D. Claxton and R.C. Staunton. Measurement of the point-spread function of a noisy imaging system. Optical Society of America Optics Info Base, 25:159-170, 2007. 
[48] J.W. Choi, M.G. Kang, and K.T. Park. An Algorithm to Extract Camera-Shaking Degree and Noise Variance in the Peak-Trace Domain. IEEE Transactions on Consumer Electronics, 44(3):1159-1168, August 1998.

[49] R. Lokhande, K.V. Arya, and P. Gupta. Identification of Parameters and Restoration of Motion Blurred Images. Proceedings of the 2006 ACM symposium on Applied computing, CF:301-305, 2006.

[50] R.C. Gonzales and R.E. Woods. Digital Image Processing. Prentice Hall, New Jersey, 2nd edition, 2002.

[51] Thomas Ricker. OmniVision claims 8 megapixel OmniBSI sensor turns cellphone imaging world "upside down". http://www.engadgetmobile. com/2008/05/27/omnivision-claims-8-megapixel-omnibsi-sensor -will-turn-cellphone/, May 2008. Accessed 8 September 2008 [Online].

[52] W. Lee and J. Kim. A Cost-Effective Demosaicked Image Enhancement for a Single Chip CMOS Image Sensor. IEEE Workshop on Signal Processing Systems Design and Implementation, pages 148-153, November 2005.

[53] M. Xie. Fundamentals of Robotics: Linking Perception to Action. World Scientific, 2003.

[54] O. Yadid-Pecht and R. Etienne-Cummings. CMOS Imagers: From Phototransduction to Image Processing. Kluwer Academic Publishers Boston, 2004.

[55] O. Yadid-Pecht. Geometrical modulation transfer function for different pixel active area shapes. SPIE Digital Library, Optical Engineering, 39(4):859-865, June 2000.

[56] S.E. Umbaugh. Digital Image Analysis and Processing. CRC Press, 2005.

[57] T. Funkhouser. Image Sampling and Reconstruction. Princeton University COS426 website course notes, Fall 2000. http://www.cs.princeton.edu/courses/ archive/falloo/cs426/.

[58] A.E. Gamal and H. Eltoukhy. CMOS Image Sensors. IEEE Circuits \& Devices Magazine, May/June 2005.

[59] V. Bockaert. Digital Photography Review: Dynamic Range of a Sensor. Accessed June 202008 [Online], 1998-2008. www.dpreview.com/learn/?/key=Dynamic_ Range.

[60] J. Nakamura. Image Sensors and Signal Processing for Digital Still Cameras. CRC Press Taylor \& Francis Group, 2006. 
[61] S. Sugawa, N. Akahane, S. Adachi, et al. A 100 dB Dynamic Range CMOS Image Sensor using a Lateral Overflow Integration Capacitor. Powerpoint Presentation, 2008. http://www.ieeexplore. ieee.org/iel5/9995/32118/1494014.zip.

[62] R. Kremens. Image Processing System Applications: from Barbie Cams to the Space Telescope. Center for Imaging Science: Imaging Research Online, 2001. http:// www.cis.rit.edu/ rlkpci/PDFs/Cicc_4.PDF.

[63] P.G. Aitken. Camera Phone Obsession. Paraglyph Press, Arizona, 2004.

[64] D. Litwiller. CCD versus CMOS: Facts and Fiction. Photonics Spectra, January 2001.

[65] X. Liu. CMOS Imaging Sensors Dynamic Range and SNR Enhancement via Statistical Signal Processing. Philosophy, Stanford University, Department of Electrical Egnineering, 2002.

[66] J. Moholt, T. Willassen, et al. A 2 Mpixel 1/4-inch CMOS Image Sensor with Enhanced Pixel Architecture for Camera Phones and PC Cameras. In ISSCC Session 2, volume 2.8. IEEE International Solid-State Circuits Conference, 2008.

[67] B. Hendriks and S. Kuiper. Through a Lens Sharply. IEEE Spectrum, 2004. www. spectrum.ieee.org/print/4172.

[68] R.D. Gow, D. Renshaw, K. Findlater, et al. A Comprehensive Tool for Modeling CMOS Image-Sensor-Noise Performance. IEEE Transactions on Electronic Devices, 54(6):13211329 , June 2007.

[69] Nokia Corporation and ST Mircoelectronics. Standard Mobile Imaging Architecture. http: / /www.smia-forum. org/, 2004. Accessed 20 November 2008 [Online].

[70] G Roelofs. PNG (Portable Network Graphics) Specification, Version 1.2, Appendix: Gamma Tutorial. http://www.libpng.org/pub/png/spec/1.2/ PNG-GammaAppendix.html, January 2004. Accessed 26 June 2009 [Online].

[71] J. Zhou and J. Glotzbach. Image Pipeline Tuning for Digital Cameras. IEEE International Consumer Electronics, 2007, 20-23:1-4, June 2007.

[72] Nokia Corporation and ST Mircoelectronics. SMIA CCP2 specification. Specification 1.0 Part 2, 2004 Nokia Corporation, ST Microelectronics NV, June 2004.

[73] International Electrotechnical Commission. The IEC and colour management - RGB. Accessed July 152008 [Online], 2008. http: / / www. iec.ch/zone/colourmgmt/ cm_iecrgb.htm. 
[74] M. Stokes (Hewlett-Packard), M. Anderson (Microsoft), et al. A Standard Default Color Space for the Internet - sRGB. World Wide Web Consortium, 1996. http: //www.w3.org/Graphics/Color/sRGB.html.

[75] Wikipedia the free encyclopedia. sRGB. Wikipedia Foundation Inc., 2008. http: //en.wikipedia.org/wiki/SRGB.

[76] E.C. Cull, M.E. Gehm, D.J. Brady, et al. Dispersion multiplexing with broadband filtering for miniature spectrometers. The Optical Society of America: Optics InfoBase, 46(3):395-374, January 2007.

[77] Matt Whalen. Capturing Colour. High Definition Magazine Online, pages 37-41, 2008. Accessed 5 June 2009 [Online].

[78] D. Alleysson, S. Ssstrunk, J. Marguier. Influence of Spectral Sensitivity Functions on Color Demosaicing. Proc. ISET/SID 11th Color Imaging Conference, 11:251-257, November 2003.

[79] Wikipedia the free encyclopedia. YCbCr. Wikipedia Foundation Inc., 2008. http: //en.wikipedia.org/wiki/YCbCr.

[80] Internation Telecommucation Union. ENCODING PARAMETERS OF DIGITAL TELEVISION FOR STUDIOS. Specification Rec.ITU-R BT.601-4 Section 11B, Internation Telecommucation Union, 1994.

[81] M. Eriksson. Image Processing on limited devices. M.A.Sc., UMEA University (Sweden), Department of Computing Science, 2007.

[82] E. Hamilton. JPEG File Interchange Format. Compression Standard Version 1.02, Joint Photographic Experts Group, 1992. http://www. jpeg.org/public/jfif.pdf.

[83] F. Šroubek, J. Flusser, and G. Cristóbal. Multiframe blind deconvolution coupled with frame registration and resolution enhancement, chapter 9. P. Campisi, K. Egiazarian, 2001.

[84] C. Liu, R. Szeliski, S.B. Kang, et al. Automatic Estimation and Removal of Noise from a Single Image. IEEE Transactions on Pattern Analysis and Machine Intelligence, October 2006.

[85] A. Gersho and R.M. Gray. Vector Quantization and Signal Compression. Kluwer Academic Publishers, 1992.

[86] C. Hass. JPEG Compression, Quality and File Size. Accessed 15 July 2008 [Online], 2008. www.impulseadventure.com/photo/jpeg-compression.html. 
[87] Joseph Holmes. Portait Displays, Display Tune White Paper. 1996-2008 Portrait Displays Inc., 2008. http://www.portrait.com/us/products/dt_ colorcalibration.html.

[88] S. Hranilovic and F.R.Kschischang. A pixelated MIMO wireless optical communication system. IEEE Journal ofSelected Topics in Quantum Electronics, 12:859-874, July-Aug 2006.

[89] R. Benmouhoub O. Hammami and I. Aouadi. Exploring JPEG-2000 Entropy Coder Implementaions on XILINX VIRTEX-II PRO Platforms. Proceedings of the 2004 European Signal Processing Conference, pages 2047-2050, November 2007.

[90] The Mathworks Dcoumentation: Image Processing Toolbox, imadjust. http://www . mathworks.com/access/helpdesk/help/toolbox/images/ index.html?/access/helpdesk/help/toolbox/images/imadjust. html\&http://www. mathworks.com/cgi-bin/texis/webinator/search/ $? \mathrm{db}=\mathrm{MS} S \& \mathrm{prox}=$ page $\&$ rorder $=750 \& \mathrm{rprox}=750 \& \mathrm{rdfreq}=500 \& \mathrm{rw}$ freq $=$ $500 \& r$ lead $=250 \&$ sufs $=0$ \&order=r\&is_summary_on=1\&Resultcount $=$ $10 \&$ query=imadjust, 2008. Accessed 8 August 2008 [Online].

[91] R.C. Gonzalez and R.E. Woods and S.L. Eddins. Digital Image Processing using Matlab. Pearson Prentice Hall, 2004.

[92] The Mathworks Inc. Image Processing Toolbox. http://www. mathworks.com/ access/helpdesk/help/toolbox/images, 2008. 1994-2008 The Mathworks Inc.

[93] Cleve Moler. The Mathworks MATLAB News \& Notes. http: / / www . mathworks . com/company/newsletters/news_notes/clevescorner/spring01_ cleve.html, 2008. Accessed 8 August 2008 [Online].

[94] A. Lapidoth and P. Narayan. Reliable Communication Under Channel Uncertainty. IEEE Transactions on Information Theory, 44(6):2148-2177, October 1998.

[95] R. Raghuraman K. Krishnan, A. Mylavarapu and K. Vijayraghavan. ColorVision: A Camera Phone Color Appearance Utility. http: / scien. stanford.edu/class / psych221/projects/07/Camera_Phone_Color_Appearance_Utility_B/ psych221/Project_Report.htm, 2007. Accessed 19 January 2009 [Online].

[96] Nikkei Electronics Asia. MagnaChip Expands into Toy Market with CMOS Image Sensor SoC. http://techon.nikkeibp.co.jp/english/NEWS_EN/ $20080403 / 149977 /$, 2008. Accessed 19 January 2009 [Online]. 
[97] D.W. Scott. Multivariate Density Estimation: Theory, Practice, and Visualization. Wiley, New York, 1992.

[98] D. Endres and P. Fldik. Bayesian Bin Distribution Inference and Mutual Information. IEEE Transactions on Information Theory, 51(11):479-490, November 2005.

[99] X. T. Liu. Gray Picture Marking System. http://home.xtliu.org/projects/ gray-pic-marking/, 2007. Accessed 15 March 2009 [Online].

[100] B. L. Shoop and E. K. Ressler. An error diffusion neural network for digital image halftoning. Proceedings of the IEEE Workshop on Neural Networks for Signal Processing, pages 427-436, August-September 1995.

[101] R. T. Hoctora F. W. Wheeler and E. B. Barrett. An error diffusion neural network for digital image halftoning. Proceedings of the IEEE Workshop on Neural Networks for Signal Processing, 5674:479-490, 2005.

[102] T. Cover, J. Thomas. Elements of Information Theory, Second Edition. John Wiley \& Sons, Inc., 2006.

[103] 2007 Apple Inc. iPhone Reference Library. iPhone Application Programming Guide, Tuning for Performance and Responsiveness, 2007. Accessed 5 May 2009 [Online].

[104] PC Today: Your Mobile Authority. Mobile Processors Getting Even Better . http://www.pctoday.com/editorial/article.asp?article=articles ।

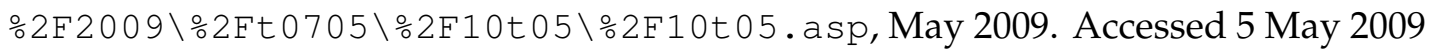
[Online].

[105] C. Van Loan. Computational Framworks for the Fast Fourier Transform. Philadelphia, PA: SIAM, 1992.

[106] E. Weisstein. Fast Fourier Transform. http://mathworld.wolfram.com/ FastFourierTransform. html, 2009. Accessed 27 June 2009 [Online].

[107] J.S. Walker. Fast Fourier Transform, 2nd Edition. Boca Raton, FL: CRC Press, 1996.

[108] R. Wang. Fast DCT algorithm. http://fourier.eng.hmc.edu/e161/ lectures/dct/node2 . html, 2007. Accessed 27 June 2009 [Online].

[109] W.-H. Chen, C. H. Smith, and S. Fralick. A Fast Computational Algorithm for the Discrete Cosine Transform. IEEE Transactions on Communications, 25(9):1004- 1009, September 1977. 
[110] Y. Arai, T. Agui, M. Nakajima. A fast DCT-SQ scheme for images. Trans. IEICE, 71(11):10951097, 1988.

[111] M. D. Wagh, H. Ganesh. A New Algorithm for the Discrete Cosine Transform of Arbitrary Number of Points. IEEE Transactions on Computers, 29(4):269-277, April 1980.

[112] P. Leea, G.-S. Liub. An efficient algorithm for the 2-D discrete cosine transform. Signal Processing, 55(2):221 - 239, 1996.

[113] Hin Lun Lee. Capacity Estimation and Characterization of a 2D Barcode Channel. Master of engineering, University of Toronto, Department of Electrical and Computer Engineering, 2008.

[114] X. Liu, D. Doermann and H. Li. A Camera-based Mobile Data Channel: Capacity and Analysis. Proceeding of the 16th ACM International Conference on Multimedia, pages 359-368, 2008.

[115] Antonio Criminisi. A Plane Measuring Device: Computing the plane to plane homography. http://www.robots.ox.ac.uk/ vgg/presentations/bmvc97/ criminispaper/node3.html, 1997. Accessed 9 April 2009 [Online].

[116] L. Jagannathan and C.V. Jawahar. Perspective Correction Methods for Camera-Based Document Analysis . Proceedings of First International Workshop on Camera Based Document Analysis and Recognition, pages 148-154, August 2005.

[117] R. Bramley. Indiana University Computer Science Department, P573 Introduction to Scientific Computing Class Notes, Least Squares Problems. http://www . cs.indiana.edu/classes/p573/notes/leastsq/leastsq.html, 2008. Accessed 9 April 2009 [Online].

[118] A. Abbasfar, D. Divsalar, K. Yao. Accumulate-Repeat-Accumulate Codes. IEEE Transactions on Communications, 55(4):692-702, April 2007.

[119] D.G. Messerschmitt J.R. Barry, E.A. Lee. Digital Communications, third edition. Springer Science+Business Media, Inc., 2004.

[120] B. Cooke. Reed-Muller Error Correcting Codes. http://www-math.mit.edu/ phase2/UJM/vol1/COOKE7FF.PDF, February 2000. Accessed 25 May 2009 [Online]. 
[121] A. Ashikhmin, S. Litsyn. Simple MAP Decoding of First-Order Reed-Muller and Hamming Codes. IEEE Transactions on Information Theory, 50(8):1812-1818, August 2004.

[122] T. K. Moon. ECE 7670, Error-Control Coding: Lecture 9 Reed-Muller codes. http://ocw.usu.edu/Electrical_and_Computer_Engineering/ Error_Control_Coding/lecture9.pdf, 2006. Accessed 1 June 2009 [Online].

[123] S. ten Brink. Code doping for triggering iterative decoding convergence. Proc. IEEE Int. Symp. Inf. Theory, page 235, July 2001.

[124] R. Urbanke T. Richardson. Modern Coding Theory. Cambridge University Press, 2008.

[125] S. Crozier, J. Lodge, P. Guinand, A. Hunt. Performance of Turbo-Codes with Relative Prime and Golden Interleaving Strategies. , page 235, July 1998.

[126] H. Wymeersch. Iterative Receiver Design. Cambridge University Press, 2007.

[127] G. Fairhurst. Cyclic Redundancy Check. http://www.erg.abdn.ac.uk/users / gorry/eg3567/dl-pages/crc.html, September 2001. Accessed 16 June 2009 [Online].

[128] S. Wicker. Error Control Systems for Digital Communication and Storage. Prentice-Hall, Inc., 1995.

[129] The UK Mobile Phone Blog (Jay). The Search for the Cameraphone Photographer of the Year. http://blog.mobiles.co.uk/2007/08/, August 2007. Accessed 18 June 2009 [Online].

[130] The Motorola Developer Network. MOTOROKR E6/E6e Developer Reference Sheet. http://developer.motorola.com/docstools/specsheets/ MOTOROKR_E6_Spec_Sheet.pdf, 2007. Accessed 13 March 2009 [Online]. 